\title{
Wide-Band Frequency Synthesizer Design with Compensation for Temperature Variations and Power Supply Noise
}

\author{
by \\ Jerry Lam \\ A thesis submitted to \\ the Faculty of Graduate and Postdoctoral \\ Affairs in partial fulfillment of the requirements \\ for the degree of
}

Doctor of Philosophy

in

Electrical Engineering

Carleton University

Ottawa, Ontario

(C) Copyright 2016

Jerry Lam 


\section{Abstract}

This thesis discusses the performance improvement frequency synthesizers, which are commonly used circuits found in many electronic systems, with the focus on alleviating the detrimental effects that temperature drifts or supply voltage changes can have. Either could require the oscillator to switch frequency bands to maintain lock, which would result in cycle slipping and phase offsets. A solution is described, involving the modification of capacitor bank switching circuits to incorporate sub-threshold switching to allow frequency band changes to occur slowly enough for the synthesizer feedback loop to track, thus preventing cycle slipping and phase offsets. This is demonstrated with a 0.009 to $1.4 \mathrm{GHz}$ frequency synthesizer implemented in a $0.18 \mu \mathrm{m}$ SiGe BiCMOS process and achieves $365 \mathrm{fs}$ integrated jitter at $1.05 \mathrm{GHz}$ with a total power consumption of $81 \mathrm{~mW}$. Measurements of the band switching circuit shows it prevents cycle slipping during band switching and reduces the maximum frequency deviation by $99.3 \%$. Measurements were conducted while changing the temperature and supply voltage, and a control voltage monitoring band switching circuit is shown which would be able to correctly switch bands as needed. The combination of these two allows a frequency synthesizer which has become resistant to transient phase effects resulting from temperature or supply voltage variations.

Following this demonstration of the core slow switching concept, further circuit enhancements were developed, including the development of a programmable bias line, allowing for controllable rise/fall times of the band switching circuit and for compensation of process, voltage, and temperature variations through calibration. The rise and fall time of the slow switching circuit can be further increased by modulating the control signal with a pulse width modulated control signal. Finally, the susceptibility of the oscillator to power supply noise is also reduced through a change in resonator circuitry, eliminating a contributor of power supply noise up-conversion in complementary cross-coupled pair oscillators. 


\section{Contents}

$\begin{array}{ll}\text { Abstract } & \text { i }\end{array}$

List of Abbreviations $\quad$ x

1 Introduction $\quad 1$

1.1 Motivation . . . . . . . . . . . . . . . . . . . 1

1.1.1 Overview of communications technology . . . . . . . . . . . . 1

1.1.2 Frequency Synthesizers . . . . . . . . . . . . . . . . . . 7

1.2 Objectives . . . . . . . . . . . . . . . . . . . . . . . . . . . . . . . . . . . .

1.2.1 Summary of Contributions . . . . . . . . . . . . . . . 13

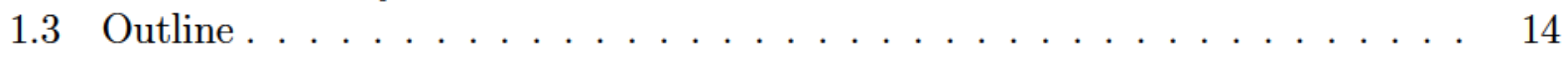

2 Background $\quad 15$

2.1 Overview of Frequency Synthesizers . . . . . . . . . . . . . . . . . 15

2.1.1 Voltage Controlled Oscillator . . . . . . . . . . . . . . 16

2.1.2 Reference Oscillator . . . . . . . . . . . . . . . . 17

2.1 .3 Divider . . . . . . . . . . . . . . . . . . . . . . . . 18

2.1.4 Phase detector and charge pump . . . . . . . . . . . . . . 18

2.1 .5 Loop Filter . . . . . . . . . . . . . . . . . . . . . . . . . . . . . . . . . . . . .

2.2 All-Digital PLLs . . . . . . . . . . . . . . . . . . . . . . . . . . . . . . . . . 21

2.3 General PLL Operation . . . . . . . . . . . . . . . . . . . . . . 24

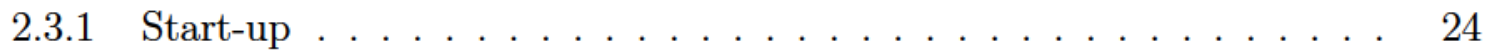

2.3.2 Locked Operation . . . . . . . . . . . . . . . . . . . . . . . . . . . . . . . . . . .

2.3.3 Cycle slipping . . . . . . . . . . . . . . . . 26

2.4 VCO Frequency Drift . . . . . . . . . . . . . . . . . . . . 27

2.5 VCO Temperature stabilization . . . . . . . . . . . . . . . . . . 29

2.6 Band gap controlled tuning . . . . . . . . . . . . . . . . . . . . . 31

2.7 Dual-Path PLL . . . . . . . . . . . . . . . . . . . . . . . . . . . . 32

2.8 Frequency Synthesizer Calibration Circuits . . . . . . . . . . . . . . 34

2.9 Summary of Background . . . . . . . . . . . . . . . . . . . . 35

3 Design $\quad 36$

3.1 Analysis of capacitor bank slow switching circuit . . . . . . . . . . . . . 36

3.1.1 Capacitor Bank Analysis . . . . . . . . . . . . . . . . . . . . 36

3.1.2 PLL System Level Analysis . . . . . . . . . . . . . . . . . . . . . 42 
3.1.3 Capacitor Bank switching circuit . . . . . . . . . . . . . . . . . 48

3.1.4 Sub-threshold limited inverter . . . . . . . . . . . . . . . . . . . 49

3.2 Alternative Solutions . . . . . . . . . . . . . . . . . . . 55

3.2.1 Multi-state PFD and charge pump . . . . . . . . . . . . 55

3.2 .2 Dynamic loop bandwidth . . . . . . . . . . . . . . . . . 56

3.2 .3 DAC generated switch control voltage . . . . . . . . . . . . 57

4 Test chip design $\quad 58$

4.1 Specifications . . . . . . . . . . . . . . . . . . . 59

4.1 .1 Pre-existing synthesizer . . . . . . . . . . . . . . . . . . . . . . 59

$4.1 .2 \quad$ Test chip . . . . . . . . . . . . . . . . . . . . . . . . . 59

4.2 Process information . . . . . . . . . . . . . . . . . . 60

4.3 Test Chip Architecture . . . . . . . . . . . . . . . . . . . . . . . . 61

4.4 Voltage controlled crystal oscillator . . . . . . . . . . . . . 63

4.5 Power supply, Voltage Regulation and References . . . . . . . . . . . . 63

4.6 Divider . . . . . . . . . . . . . . . . . . . . . . . . . . . 64

4.7 Phase detector and Charge pump . . . . . . . . . . . . . . . 67

4.8 Loop filter . . . . . . . . . . . . . . . . . . . . . . . . 6 67

4.9 Frequency Band Switching Circuit . . . . . . . . . . . . . . . . . 68

4.10 Voltage-Controlled Oscillator . . . . . . . . . . . . . . . . . . . . . . 69

4.10 .1 Oscillator Specifications _. . . . . . . . . . . . . . . . . 69

4.10 .2 Oscillator topology and Power Supply Noise . . . . . . . . . . . . 70

4.10 .3 LC resonator design and Tank Inductor . . . . . . . . . . . . . . 74

4.10 .4 Capacitor bank and varactors . . . . . . . . . . . . . . . . . . . 79

4.10 .5 Gain circuitry and Bias circuitry . . . . . . . . . . . . . . 83

4.10 .6 Output buffer . . . . . . . . . . . . . . . . . 86

4.10 .7 VCO Simulation Results . . . . . . . . . . . . . . . . . 88

4.11 Overall Chip Layout . . . . . . . . . . . . . . . . . . . . . 93

4.12 Unresolved Issues . . . . . . . . . . . . . . . . . . . . . . . . . . 96

$\begin{array}{lll}5 & \text { Measurement results } & 97\end{array}$

5.1 Test Setup . . . . . . . . . . . . . . . . . . . . . . . . . . . 97

5.2 General PLL Operation $\ldots \ldots \ldots 9$

5.3 Temperature Measurements . . . . . . . . . . . . . . . . . . . . . . 103

5.4 Frequency Band switching circuit $\ldots \ldots \ldots \ldots \ldots$

6 Circuit Improvements 111

6.1 Diode line calibration . . . . . . . . . . . . . . . . . . . . . . . . 111

6.2 Control line dithering . . . . . . . . . . . . . . . . . . . . 113

6.3 Power supply noise rejection in complementary VCOs . . . . . . . . . . 115

6.3 .1 Noise Analysis and Simulation . . . . . . . . . . . . . . . . . 117

6.3.2 Test chip architecture and specifications . . . . . . . . . . 120

6.3 .3 Process . . . . . . . . . . . . . . . . . . . . . . . . . . . . . . . . . . . . . . . . 121

6.3 .4 Shared test circuitry . . . . . . . . . . . . . . . . . . . . . . . . 121

6.3 .5 Oscillator variants . . . . . . . . . . . . . . . . . . . . . 123 
6.3.6 Transformer layout and characterization . . . . . . . . . . . . . 126

6.3 .7 Test chip layout . . . . . . . . . . . . . . . . . . . 128

6.3.8 Simulated frequency range, phase noise and power consumption . . . 130

6.4 Summary . . . . . . . . . . . . . . . . . . . . . . 131

7 Conclusion $\quad 135$

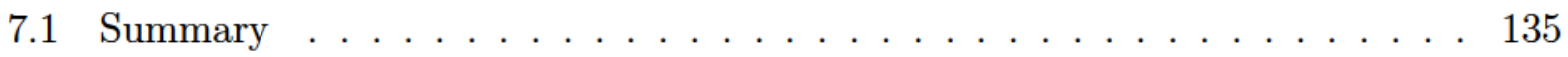

7.2 Contributions . . . . . . . . . . . . . . . . . 137

7.3 Future work . . . . . . . . . . . . . . . . . . . . 138 


\section{List of Figures}

1.1 Estimated internet traffic per month in $\mathrm{PB} /$ month (petabytes/month) [1] . . 2

1.2 Data rate for various optical, wireline and wireless communication link types 2

1.3 Approximate transistor transit frequency for commercial processes . . . . . . 4

1.4 Integrated circuit sales figures over time and sorted by application, showing the dominance of digital applications in terms of market share . . . . . . 5

1.5 Plot of simulated PLL frequency over time comparing linear and non-linear responses to a frequency transient . . . . . . . . . . . . 8

1.6 Plot of simulated PLL showing the frequency deviation with 4 different frequency shift values . . . . . . . . . . . . . . . 9

1.7 Plot of simulated PLL showing the cycle offset with 4 different frequency shift

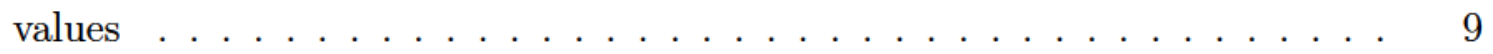

1.8 Example constellation diagram of a QPSK modulated signal . . . . . . . . 11

1.9 Example of the reaction of a QPSK system to a symbol error and a phase offset. 11

1.10 Example constellation diagram of a DQPSK modulated signal . . . . . . . . 12

1.11 Example of the reaction of a DQPSK system to a symbol error and a phase

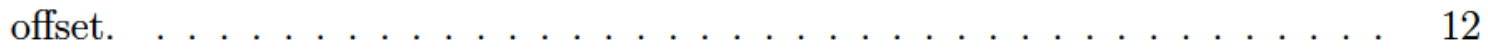

2.1 System diagram of a typical PLL . . . . . . . . . . . . . . . 16

2.2 Schematic of a typical tri-state phase-frequency detector $\ldots \ldots \ldots 19$

2.3 Schematic of a typical tri-state phase-frequency detector . . . . . . . . 20

2.4 Schematic of a typical PLL loop filter . . . . . . . . . . . . . . . . 21

2.5 System diagrams of 2 possible configurations of an all-digital PLL . . . . . 22

2.6 Laplace system diagram of a PLL . . . . . . . . . . . . . . . 25

2.7 Plot of the simulated upper and lower tuning voltage of a frequency band of a VCO over temperature . . . . . . . . . . . . . . . . . 28

3.1 Schematic showing typical VCO capacitor bank circuits . . . . . . . . . . . 38

3.2 Simplified model of a capacitor bank circuit . . . . . . . . . . . . . 38

3.3 Pole-zero plot of the capacitor bank . . . . . . . . . . . . . . . . . . 40

3.4 Simplified model of the capacitor bank . . . . . . . . . . . . . . . . 40

3.5 Laplace domain system diagram of the PLL used to analyze its behaviour with slow band switches . . . . . . . . . . . . . . . . . 43

3.6 Schematic showing typical VCO capacitor bank circuits . . . . . . . . . . . . 43

3.7 Plot of the simulated scaled transient phase and frequency errors with conventional band switching and slow switching with varying filter bandwidths . 
3.8 Plot of the attenuation of maximum phase and frequency errors with different filter bandwidths . . . . . . . . . . . . . . . . . . 48

3.9 Schematic of the capacitor bank slow switching circuit . . . . . . . . . 51

3.10 Comparison of the theoretical and simulated current in a chain of diode connected MOSFETs . . . . . . . . . . . . . . . . . . 52

3.11 Comparison of the theoretical and simulated rise/fall time of the sub-threshold current limited inverter with varying number of diode connected transistor in the bias line . . . . . . . . . . . . . . . . . . . 53

3.12 Design of a expanded multi-state phase-frequency detector . . . . . . . . 56

4.1 I-V curves of a 20 finger $20 \mu \mathrm{m}$ NMOS transistor in the BiCMOS process used to implement the chip . . . . . . . . . . . . . . . . . .

4.2 Basic simulated RF parameters of a 20 finger $20 \mu \mathrm{m}$ NMOS transistor in the BiCMOS process used to implement the chip. The large $f_{\max }$ and low NFmin values likely indicate poor modelling of gate resistance. . . . . . . . . . 62

4.3 System diagram of the test chip . . . . . . . . . . . . . . . . 63

4.4 Schematic of the voltage controlled crystal oscillator used as the frequency reference on the test chip . . . . . . . . . . . . . . . . . . . 64

4.5 Schematic of the LDO regulator used to generate the regulated power supplies 65

4.6 Plot of the simulated LDO output noise voltage . . . . . . . . . . . . 65

4.7 Schematic of the overall architecture of the frequency divider . . . . . . . 66

4.8 Schematic of a CML latch using bipolar transistors . . . . . . . . . . . 66

4.9 Schematic of a CMOS TSPC divider . . . . . . . . . . . . . 67

4.10 Schematic of the charge pump . . . . . . . . . . . . . . . . 68

4.11 Schematic of (a) a typical PMOS cross-coupled pair VCO and (b) simplified single ended version illustrating the common mode noise path . . . . . . . 71

4.12 Small signal equivalent circuit of the PMOS cross-coupled pair VCO showing noise path for (a) power supply noise and (b) ground noise . . . . . . . . 72

4.13 Schematic of (a) a typical CMOS cross-coupled pair VCO and (b) simplified single end version illustrating the common mode noise path . . . . . . . . .

4.14 Small signal equivalent circuit of the CMOS cross-coupled pair VCO showing noise path for (a) power supply noise and (b) ground noise . . . . . . . . 74

4.15 Plot comparing simulated inductor parameters for different inductor geometries 79

4.16 Plot of the simulated inductor parameters for the VCO tank inductor . . . . 79

4.17 Schematic of the capacitor bank circuit . . . . . . . . . . . . . 80

4.18 Plot of the simulated equivalent parallel capacitance and parasitic resistance (at $4 \mathrm{GHz}$ ) of the VCO capacitor banks over intermediate switch voltages . . 81

4.19 Schematic of the $\mathrm{VCO} \ldots \ldots$. . . . . . . . . . . . . . . . . . . . . . . . . . . 83

4.20 Schematic of the $\mathrm{VCO} \ldots \ldots \ldots 6$

4.21 Schematic of the current mirrors used to bias the VCO, the VCO buffer and the CML driver . . . . . . . . . . . . . . . . . . . . . 87

4.22 Schematic of the PMOS source follower used as the VCO buffer $\ldots . . .87$

4.23 Schematic of the CML driver circuit and divider input amplifier used to connect VCO and divider circuit blocks. 
4.24 Plot of the simulated frequency vs frequency band code setting at high and low control voltages at hot, nominal and cold temperatures with the lowest current setting GM0 . . . . . . . . . . . . . . . . . . .

4.25 Plot of the simulated frequency vs frequency band code setting at high and low control voltages at hot, nominal and cold temperatures with the current setting GM1 . . . . . . . . . . . . . . . . . . . .

4.26 Plot of the simulated frequency vs frequency band code setting at high and low control voltages at hot, nominal and cold temperatures with the current setting GM2 . . . . . . . . . . . . . . . . . . . . .

4.27 Plot of the simulated frequency vs frequency band code setting at high and low control voltages at hot, nominal and cold temperatures with the highest current setting GM3 . . . . . . . . . . . . . . . . . .

4.28 Plot of the simulated phase noise at a $1 \mathrm{MHz}$ offset vs frequency for different bank, control voltage and current settings . . . . . . . . . . . .

4.29 Plot of the simulated VCO figure of merit vs frequency for different bank, control voltage and current settings . . . . . . . . . . . . . . . . . . . 92

4.30 Plot of the simulated VCO vs offset frequency over different process corners . $\quad 92$

4.31 Photograph of the manufactured test chip . . . . . . . . . . . . . . . . . . . 94

4.32 Photograph of a sample printed circuit board used to measure the test chip . 95

5.1 Diagram showing the test setup for most of the measurements of the PLL . . 98

5.2 Plot of the integrated jitter vs. frequency for different VCO frequencies . . .

5.3 Plot of of the phase noise of the PLL in integer-N mode with an output frequency at $1.05 \mathrm{GHz}$. . . . . . . . . . . . . . . . . 100

5.4 Plot of the integrated jitter of the PLL over its operating range . . . . . . . 100

5.5 Plot of the PLL phase noise across its frequency range . . . . . . . . . . . . 101

5.6 Spectrum plot showing PLL out-of-band spurs in integer-N mode with an output frequency at $1.05 \mathrm{GHz}$. . . . . . . . . . . . . . . . . . 102

5.7 Spectrum plot showing PLL in-band spurs in fractional-N mode with an output frequency at $1.0500005 \mathrm{GHz}$. . . . . . . . . . . . . . . . . . . . . 102

5.8 Proposed feedback circuit for band switching control based on control voltage monitoring . . . . . . . . . . . . . . . . . . . . . . . 104

5.9 VCO Control voltage and output frequency of the frequency synthesizer with changing temperature. . . . . . . . . . . . . . . . . 105

5.10 VCO Control voltage and output frequency of the frequency synthesizer with changing supply voltage. . . . . . . . . . . . . . . . .

5.11 Plot of frequency deviation vs. time of the synthesizer (which is set to 58 $\mathrm{MHz}$ ) while undergoing a VCO bank switch using regular switching and slow switching. Measured, simulated and theoretical results are shown. . . . . . .

5.12 Plot of the average spectrum of the synthesizer output over a 1 minute period with no band switching, with regular band switches at random intervals, and with slow band switching at random intervals. . . . . . . . . . . . . . . . . 110

6.1 Schematic of a diode chain with programmable current mirror and switched

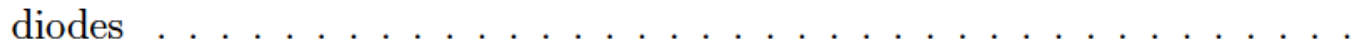


6.2 Schematic of a diode line calibration circuit . . . . . . . . . . . . 113

6.3 Plot of the rise/fall times of the sub-threshold limited buffers with different process corners and supply voltages both with and without calibration . . . . 114

6.4 Plot of the rise/fall times of the sub-threshold limited buffers with different process corners and temperatures both with and without calibration . . . . 114

6.5 Plot of a buffer output . . . . . . . . . . . . . . . . . . . 115

6.6 Simplified schematics of the proposed noise rejection CMOS VCOs . . . . . 116

6.7 Simplified small signal model of the RC noise rejection VCO . . . . . . . 118

6.8 Simplified small signal model of the transformer noise rejection VCO $\ldots 118$

6.9 Simulated phase noise of a VCO prototype showing the effect of power supply noise on the RC noise rejection VCO phase noise . . . . . . . . . . . . 119

6.10 Simulated phase noise of a VCO prototype showing the effect of power supply noise on the transformer noise rejection VCO phase noise . . . . . . . . . 120

6.11 Schematic of the open drain VCO buffer connected to the test setup . . . . . 122

6.12 Simulation results of the oscillator buffer . . . . . . . . . . . . . . . 122

6.13 Schematic of the VCO capacitor banks . . . . . . . . . . . . . . . 123

6.14 Schematic of the first VCO variation . . . . . . . . . . . . . . . . . . 124

6.15 Schematic of the first VCO variation . . . . . . . . . . . . . . . . . 125

6.16 Schematic of the second VCO variation . . . . . . . . . . . . . . 125

6.17 Schematic of the third VCO version . . . . . . . . . . . . . . 126

6.18 Layout of the transformer. The width and height are each $300 \mu \mathrm{m}$. . . . . 127

6.19 Plot of the transformer characterization simulation results . . . . . . . . 128

6.20 Simulated resonator impedance (real and imaginary) for the transformer coupled VCO . . . . . . . . . . . . . . . . . . . . . . . . . . 129

6.21 Layout diagram of the test chip . . . . . . . . . . . . . . . . 130

6.22 Simulated frequency tuning range of the three oscillator versions . . . . . . 131

6.23 Simulated phase noise (at a $1 \mathrm{MHz}$ offset) of the three oscillator versions . . 132

6.24 Simulated DC power consumption of the three oscillator versions . . . . . . 132

6.25 Simulated oscillator figure of merit of the three oscillator versions . . . . . 133

6.26 Simulated oscillator power supply noise coupling to varactor port for the 3 oscillator versions . . . . . . . . . . . . . . . . . 133 


\section{List of Tables}

4.1 Comparison of simulated inductor parameters using IBM kit models . . . . 78

4.2 Size of component values used in the VCO capacitor banks . . . . . . . . 80

4.3 Simulated VCO performance at different process corners $\ldots \ldots$. . . . . . 93

5.1 Comparison of performance to other published works _ . . . . . . . . 103 


\section{List of Abbreviations}

AC Alternating Current

ADC Analog-to-digital converter

ADPLL All-digital phase-locked loop

AFC Automatic frequency calibration

AMOS Accumulation-mode metal-oxide-semiconductor

BiCMOS Bipolar and complementary metal-oxide-semiconductor

BIST Built-in self-test

CDR Clock and data recovery

CML Current-mode logic

CMOS Complementary metal-oxide-semiconductor

CP Charge pump

CTAT Complementary to absolute temperature

DAC Digital-to-analog converter

DC Direct current

DCO Digitally controlled oscillator

DFT Discrete Fourier Transform

DIBL Drain-induced barrier lowering

DQPSK Differential quadrature phase-shift keying

DSL Digital subscriber line

DSP Digital signal processing

EM Electromagnetic 
FinFET Fin field effect transistor

FET Field effect transistor

GAA Gate-all-around

GHz Gigahertz

Gm Transconductance

IC Integrated circuit

I2C Inter-integrated circuit

IoT Internet of things

IP Internet Protocol

ISSCC International Symposium of Solid-State Circuits

I-V Current-voltage

kHz kilohertz

KVCO Voltage controlled oscillator control voltage to frequency gain

LC inductor-capacitor

LDO Low-dropout

LVS Layout vs. schematic

MASH Multi-stage noise shaping

MEMS Micro-electronic mechanical system

$\mathrm{MHz}$ Megahertz

MIM Metal-insulator-metal

MOM Metal-oxide-metal

MOS metal-oxide-semiconductor

MOSFET metal-oxide-semiconductor field effect transistor

MPW Multi-project wafer

NMOS n-type metal-oxide-semiconductor

OFDM Orthogonal frequency division multiplexing

PB Petabytes 
PCB Printed circuit board

PFD Phase-frequency detector

PLL Phase-locked loop

PMOS p-type metal-oxide-semiconductor

POTS Plain old telephone system

PSRR Power supply rejection ratio

PTAT Proportional to absolute temperature

ppm Parts per million

QAM Quadrature amplitude modulation

QFN Quad flat no-leads

QPSK Quadrature phase-shift keying

RC Resistor-capacitor

RLC Resistor-inductor-capacitor

RF Radio frequency

SAW Surface acoustic wave

SERDES Serializer-deserializer

SiGe Silicon-Germanium

SMA Sub-miniature version A

SOI Silicon-on-insulator

TDC Time-to-digital converter

TSPC True single-phase clock

VCO Voltage controlled oscillator

VCXO Voltage controlled crystal oscillator 


\section{Chapter 1}

\section{Introduction}

\subsection{Motivation}

\subsubsection{Overview of communications technology}

High speed communications have become increasingly prominent in recent years. Increasing data rates have enabled a myriad of different services, such as social media, streaming video, cloud computing and over-the-top content services, in addition to more traditional uses such as file transfer, e-commerce, email, etc. This, in turn, has lead to increased demand for higher data rates and larger bandwidth, not only by increased number of users and increased use by existing users, but also by demands for higher fidelity media, such as high resolution images, or videos at high frame rates with lossless compression algorithms. Increased data rates in turn enables new applications that might have previously been impractical.

A glimpse of the importance digital communications has had can be seen by looking at the volume of internet traffic. Over the past few decades, internet traffic has grown by incredible amounts. Fig. 1.1 shows estimates and projections for past and future data rates of IP traffic based on [1]. Usage has expanded at a tremendous growth rate and will likely continue further [2].

Fig. 1.1 also shows that mobile internet traffic is increasing at a high rate, gradually making up an increasingly larger portion of total internet traffic, despite the approaching market saturation of the smartphone market. Future increases in traffic will likely come from greater use of over-the-top content services with higher fidelity media, in addition to the rise of wearable devices (such as smart watches), and the continued development of the "internet of things" (IoT), which hopes to see everyday items such as appliances, furniture, tools, vehicles, etc. connected to the internet for potentially increased functionality [3].

The increased demand for data has put tremendous demands for higher data rates for 


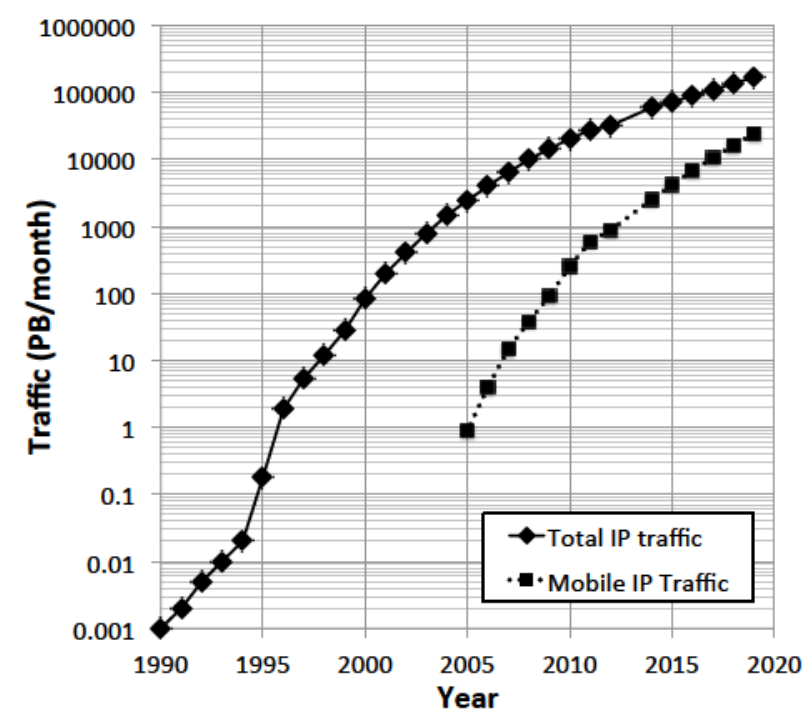

Figure 1.1: Estimated internet traffic per month in PB/month (petabytes/month) [1]

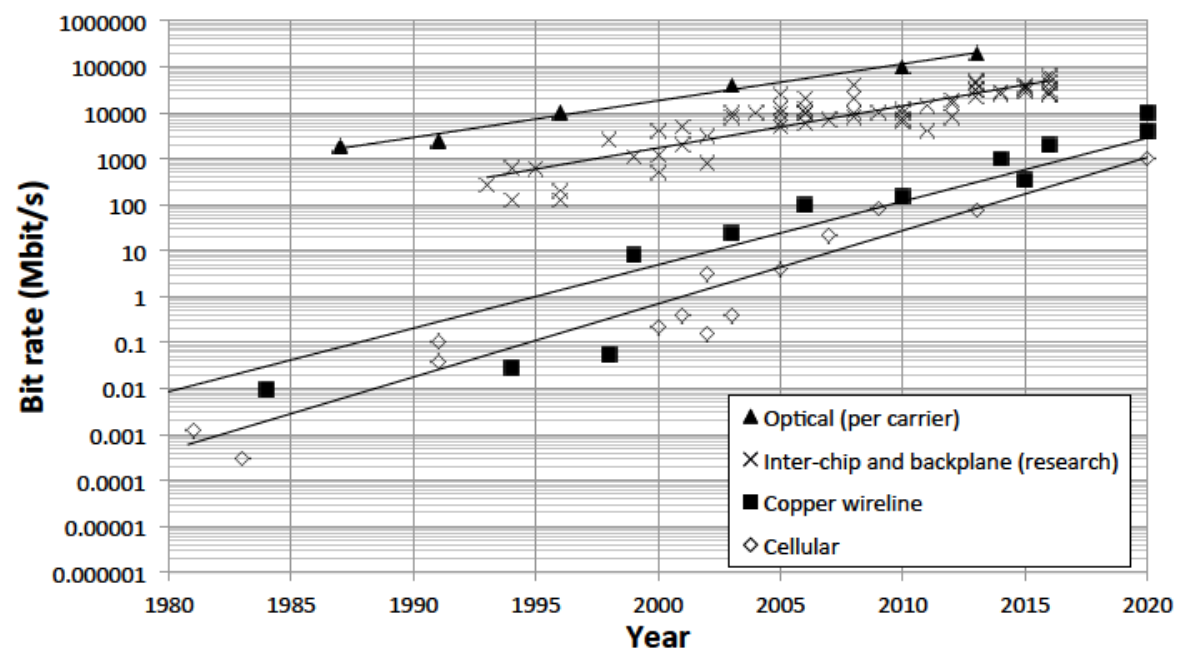

Figure 1.2: Data rate for various optical, wireline and wireless communication link types 
all types of communications links, such as wireless (for end user applications), copper wireline (for interconnect and for short distance communications) and optical (used for network backbones), all of which have seen drastic improvements in capabilities, with future improvements projected for the near future. The vast improvements in performance over the past few decades can be seen in Fig 1.2, which shows maximum data rates for wireless cellular standards, copper wireline (for POTS and DSL) [4], commercial optical systems [5], and PCB back-plane transceivers (based on research published in ISSCC since 1990), all of which have shown exponential growth in transmission rate.

Increased availability of high speed backbones and wireless links in turn allows for a more diverse set of services and content to be offered, which in turn fuels demand for greater bandwidth. This cycle is likely to continue into the near future until some limiting point is reached, such as physical limits for communications systems (such as the non-linear Shannon limit), prohibitively increased costs, or decreased consumer or business interest.

While improvements in communication systems have focussed on increased bitrate in response to the demand for increased usage, there is also a push to improve the performance of communications systems in many ways. For instance, software defined networking [6] seeks to abstract network infrastructure connections through software controls. This necessarily adds higher constraints to the design of the hardware to accommodate this, since the hardware can no longer be optimized for any given purpose, since it may be reconfigured at any time.

Radio transceivers face additional pressure with each generation of technology, such as new modulation formats with higher complexity and greater bandwidths, while still requiring backwards compatibility with legacy systems. The development of cognitive radio [7], which seek to dynamically search for unused wireless bandwidth and adapt communications protocols accordingly, further increases the challenges in designing wireless communications circuits. A potential avenue is the development of software defined radios (which rely heavily on algorithms implemented in software or reprogrammable hardware), or reconfigurable multi-band radios (which use circuits which can work in multiple frequency bands or modes of operation), but result in vastly increased challenges in the circuit design. Other challenges will face communications systems of all types, such as demand for improved encryption and security, more software interfaces to control operation dynamically, while combined with reduced cost and power consumption.

Much of the performance improvements of communications systems in recent times can be credited to improvements in the process technology used to manufacture them, typically through the geometric scaling of silicon CMOS, which is used for its low cost and ease of scalability. As transistors are made smaller, the overall transistor density can be increased, reducing cost per transistor, parasitic capacitance and increasing transistor density, simulta- 


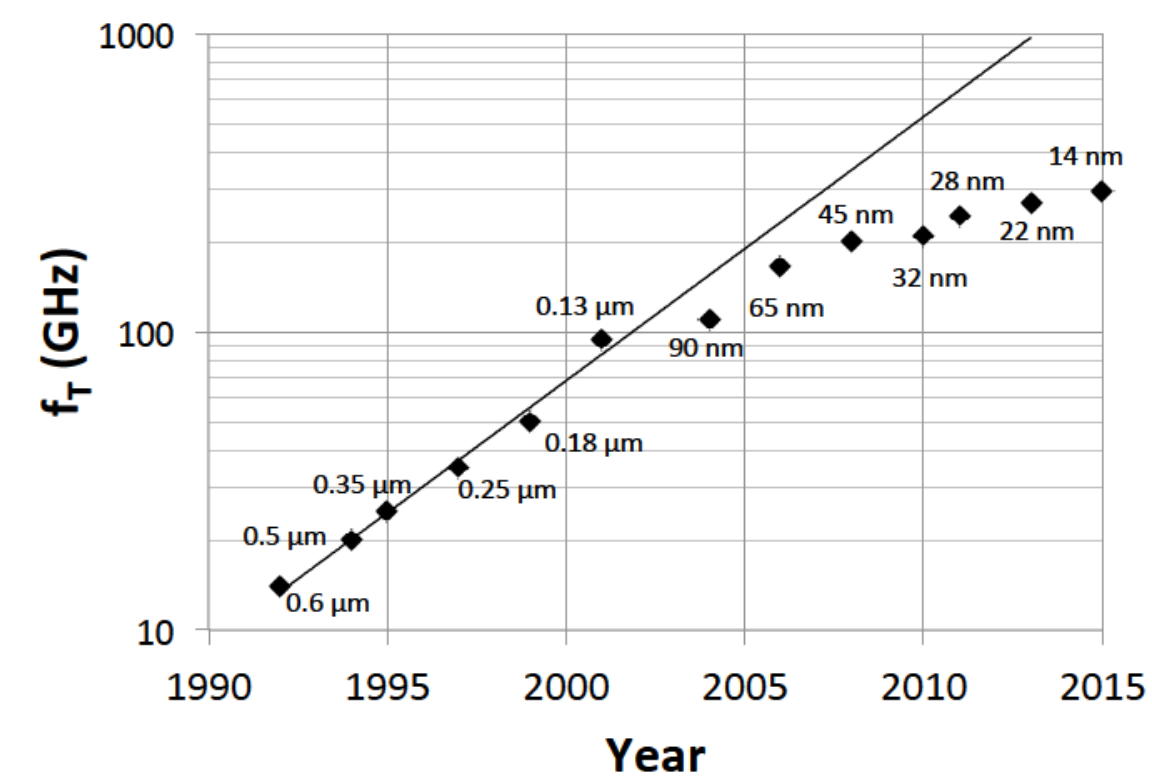

Figure 1.3: Approximate transistor transit frequency for commercial processes

neously increasing potential functionality and performance while reducing cost, form factor and power consumption. Thus, transistor scaling has allowed a simultaneous improvement in multiple dimensions (cost, functionality, speed and power consumption) all at the same time.

However, improvements in CMOS processes do not provide a complete picture of the nature of performance increases. Fig. 1.3 shows a plot of approximate transistor $f_{T}$ for various commercial silicon processes over time. While rapid improvements have been seen between 1990 to 2002, the rate of improvement has decreased since then. Currently, one of the highest experimental $f_{T}$ for a silicon process is still at $485 \mathrm{GHz}$ using a $45 \mathrm{~nm}$ SOI CMOS transistor, which was reported in 2007 [8]. The reduced performance benefits from geometric scaling is due to many reasons, such as the emphasis on digital gate density over analog or RF performance, or the greater contribution that wiring parasitics (such as series metal resistance, via resistance, inter-metal capacitance) have on overall circuit performance, which do not see as much improvement with geometric scaling. Short channel effects and other effects, such as drain-induced barrier lowering (DIBL) and quantum barrier tunnelling have become more significant with smaller geometries, making process scaling more difficult, while the costs associated with manufacturing small geometry CMOS wafers has continued to rise.

The use of FinFETs is being used to help alleviate some of the issues encountered with process scaling, but at significantly increased process costs. Although it is predicted that 


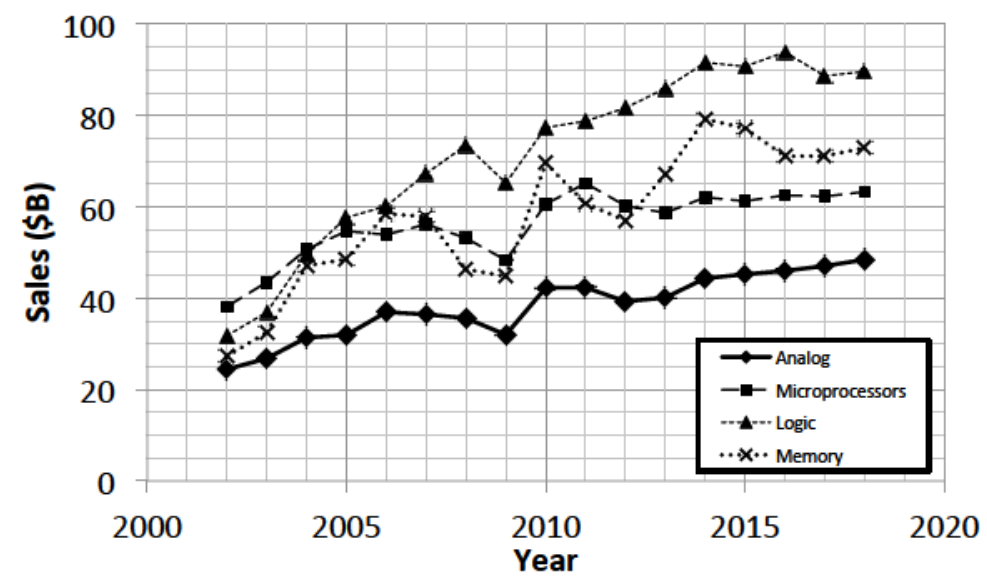

Figure 1.4: Integrated circuit sales figures over time and sorted by application, showing the dominance of digital applications in terms of market share

continued geometric scaling will allow for overall decreased cost per transistor and improved system performance through increased transistor density [9], the parasitics associated with the fin structures tends to reduce the high frequency performance gains achieved by the reduction in geometry $[10,11]$, further slowing down any potential gains in analog or $\mathrm{RF}$ performance. The use of fully depleted silicon-on-insulator (FDSOI) transistors has also been growing in popularity. While the targeted application for FDSOI tends to be low power digital logic (due to the ability to dynamically modulate device threshold voltages to reduce leakage power or improve performance), analog and RF circuits may see some benefits from reduced parasitics, and the ability to use well biasing to control performance.

The nature of transistors used into the deep nanometer range is somewhat unclear. Due to the increasing number of non-idealities encountered with each process node, it is clear that geometric process scaling cannot continue indefinitely, with a possible plateau highly likely around $3 \mathrm{~nm}$ [12]. Without fundamental changes to the nature of the transistor itself, such as the use of graphene [13], the use of nano wires or gate-all-around (GAA) structures [14], III-V materials or Ge as channel materials [15], tunnel FETs[16], or Spintronic devices $[17,18]$, it may be difficult to maintain constant improvements in performance or transistor density. The use of other types of processes (such as BiCMOS, or III-V processes) can resolve some issues but have their own drawbacks such as poor logic density and significantly higher cost.

The scaling of CMOS processes has not led to proportional benefits for analog and RF designs, due to differing requirements between analog applications and digital applications. The vast majority of IC sales result from digital ICs, as can be seen in Fig. 1.4, which shows integrated circuit sales over time separated by application according to information published 
by the World Semiconcutor Trade Statistics [19]. Analog ICs account for fewer sales than for any of the 3 major digital IC applications, and are thus dwarfed by their total. Since the vast majority of designs are digital circuits, they are the main drivers behind CMOS process scaling. Thinner gate oxides has led to the use of lower supply voltages, making analog designs using multiple stacked transistors (such as high impedance current sources or Gilbert cells) less feasible, while non-linear short channel effects become worse with smaller process nodes. Noise properties also do not necessarily improve as density increases, and can often worsen due to higher parasitic resistance and greater coupling between signal lines. Scaling of designs is also not feasible for practical reasons in many situations. For instance, in RF designs, transmission frequencies are restricted to certain bands due to regulatory reasons, while the Friis transmission equation shows that wireless link range decreases with higher frequencies, decreasing range. Since the frequency does not scale, the sizes of passive components and resonant circuits remains the same, limiting possible area reduction. Improvements have thus been made in the circuit topologies themselves, such as relying less on stacked transistors and using charge domain or time domain (rather than voltage domain) to represent signals, in addition to earlier conversion from analog to digital, to allow processing to be done in DSP instead [12,20]. However, these techniques have limits of their own. For instance, analog-digital conversion also still requires the presence of a high performance analog circuit, since it becomes the limiting factor in determining how clean the digital signal is from noise, interference and distortion.

Thus, in order to obtain the exponential improvements in link performance in recent years (as shown in Fig. 1.2), improvements in the system level have also been needed. This has led to the development of technologies such as channel equalization, distortion compensation, more powerful compression and error correction codes, multiple carrier modulation (such as OFDM), and the use of multiple antennas, among many others. However, as with process scaling and improvements in circuit topologies, many of the simpler technologies that have been easy to implement have already been developed. Future improvements will thus be more complex, requiring greater computational power and more knowledge about the communication channel, leading to standards that become increasingly more complex and difficult to implement. In order to continue gains in performance, improvements in all of these areas will likely be required, from system level improvements to continued leveraging of process scaling, and improvements in circuit design. 


\subsubsection{Frequency Synthesizers}

As a result of the high demands placed upon them, communications systems have become highly specialized and complex electronic systems. To manage design complexity and to allow design reuse, communications systems are generally made up of numerous standardized conventional sub-blocks, such as amplifiers, up/down converters, data converters, retiming circuits, clock trees, etc. One of the benefits this design partitioning has is to allow research into improvements for the various sub-blocks to benefit many different applications at once.

While there are numerous areas in which communications systems may be improved, this thesis focusses on a critical circuit used in communication circuits of all types: the frequency synthesizer. Frequency synthesizers are circuits which produce a waveform of a specific frequency. They are used in many places in many different types of systems such as local oscillators for up-conversion/down conversion in RF systems, sampling clocks in data converters, timing recovery in clock-data recovery (CDR) circuits, timing circuits for synchronization applications, etc. Due to the wide range of uses, frequency synthesizers have a diverse set of requirements, such as tuning range, jitter/phase noise, lock acquisition time, power consumption, etc., all of which vary from application to application.

The implementation of frequency synthesizers can take many forms, such as a mixer based circuit (where waveforms are multiplied, divided down or mixed in various proportions), direct digital synthesis (which uses digital-to-analog conversion to generate the output waveform), but one of the most common implementation uses a phase-locked loop (PLL). A PLL generates its output using a voltage-controlled oscillator (VCO) which generates a periodic waveform whose period is controlled by a control port voltage. The PLL can set the output frequency very accurately by monitoring the phase of the VCO and comparing it to a reference signal, adjusting the control voltage as necessary.

\subsubsection{Cycle slipping and Phase Offsets}

Due to their critical importance in systems of all types, there is considerable research interest into improving the performance of the PLL, from improving its capabilities. One important issue regarding PLL performance is the issue of preventing cycle slips. A cycle slip occurs when the PLL output phase is shifted by an amount large enough to take the PLL out of its linear range. While this can occur for many reasons, the most frequent reason is due a change the the PLL oscillator frequency band, which may occur in response to temperature drifts or changes in supply voltage. An example of cycle slipping is shown with the frequency vs. time plot of a simulated PLL, as shown in Fig. 1.5. At time $t=0$, a frequency transient of size 1 is inserted into the PLL, and the response to this offset can be seen with both linear 


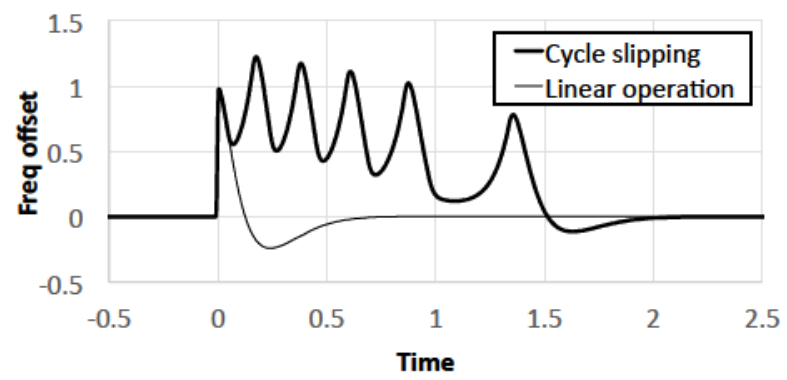

Figure 1.5: Plot of simulated PLL frequency over time comparing linear and non-linear responses to a frequency transient

operation and with non-linear cycling slipping. In linear operation, the PLL can recover rapidly from the frequency error, as its response is governed by conventional linear systems theory, while with cycle slipping, a much longer time is needed. While the negative feedback portion of the loop is able to lock the output phase to the reference signal, a permanent phase offset remains, with the output PLL phase shifted by an integer multiple of $2 \pi$ relative to its original phase.

To demonstrate the problems that cycle slipping can cause a PLL is simulated at the system level. Fig. 1.6 and 1.7 show the results from a simulation of a 1.5 GHz PLL locked to a $30 \mathrm{MHz}$ signal, with a loop bandwidth of $100 \mathrm{kHz}$ with a frequency step taking place at $\mathrm{t}=0$ in 4 cases: Case 1 is a baseline with no frequency step, Case 2 is a case with small frequency step that does not result result in cycle slipping, Case 3 is a slightly larger frequency step which results in moderate cycle slipping while Case 4 is a case where a frequency step causes significant cycle slipping. Figure 1.6 shows the frequency deviation for each case. Note that while the size of the frequency steps in cases 3 and 4 are only $25 \%$ and $50 \%$ larger than that in case 2 respectively, the time to regain lock is increased by $50 \%$ and $200 \%$ respectively. More significant is the results shown in Figure 1.7, which shows the difference in number of VCO cycles from the baseline case 1 . With case 2 , the output is off by a considerable number of cycles, but returns to its original phase. Case 3 and case 4 however lock to a different phase upon regaining PLL lock, indicating that the output is off by a large number of cycles.

Cycle slipping and the resulting phase offsets can cause many problems, depending on the application in which the frequency synthesizer is used. A few potential examples are listed here. Note that in all of these cases, the system always has a method of regaining synchronization, since it must be able to synchronize the clocks and timing upon startup, but the time it takes for it to to be determined that there is a loss of synchronization can be 


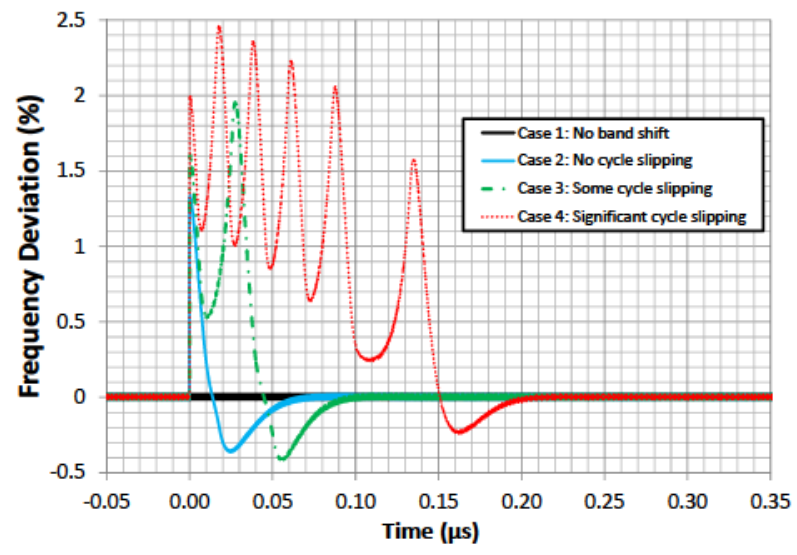

Figure 1.6: Plot of simulated PLL showing the frequency deviation with 4 different frequency shift values

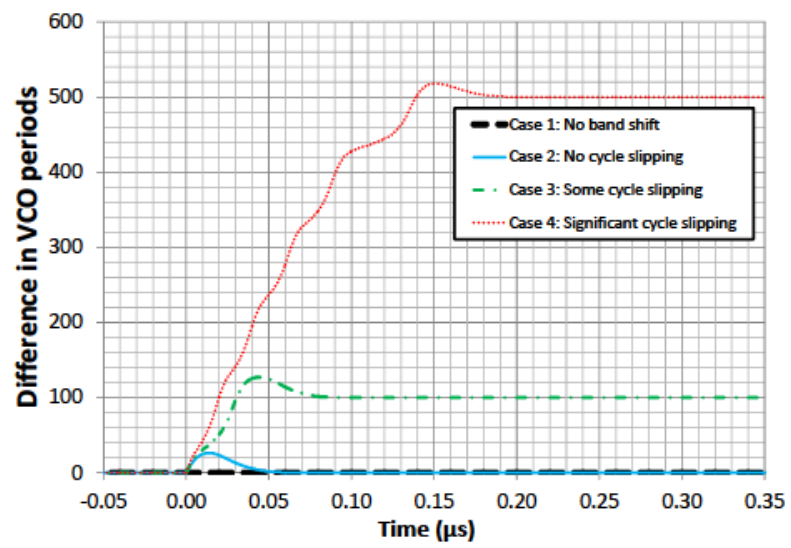

Figure 1.7: Plot of simulated PLL showing the cycle offset with 4 different frequency shift values 
significant, and the loss of use of the system during this amount of time, in addition to the time needed to resynchronize the system may be unacceptable.

- High speed optical and wireline communications: High speed fibre optic communication systems are ubiquitous in many applications, such as tier one network backbones and in data center interconnect, due to the high rate of data transfer possible with low bit error rate over very long range. These applications require very high up-time with very little tolerance for faults or errors. Thus, optical communication electronics are held to very high standards for reliability, particularly for the system clocks. Cycle slipping in a clock will cause loss of data for a brief period and the resulting phase offset afterwards will cause a loss of synchronization, requiring a more costly system reset operation, which is generally not acceptable due to the stringent reliability requirements.

This is particularly true for coherent optical systems, which rely on very precise optical phase modulation to transfer data at high rates. High speed serial data communications circuits have similar issues, although the reliability requirements may be relaxed, depending on the application.

- Low power sensor nodes: Low power RF CMOS circuits have made possible wireless sensor networks and low power sensor nodes connected to the internet of things. To further reduce system power and improve battery life (or to allow the use of scavenged power systems) RF transceivers, the low power nodes are put to sleep with periodic wakeup times to transfer data. For maximum efficiency, the sleep-wakeup cycles must be accurately timed and coordinated between transmitting and receiving pairs, with strict requirements for clock accuracy [21]. Thus, a phase offset resulting from cycle slipping would break this cycle and potentially cause problems, wasting energy in extra resynchronization steps. This is compounded if multiple nodes simultaneously lose synchronization, since they may attempt to transmit over top of each other.

- Spread spectrum communications: Spread spectrum communications uses techniques to allow a signal to be transmitted with a wide bandwidth, lowering the spectral density, and can allow things such as transmission below the noise floor. This can be useful for secure communication, and may have potential future applications in cognitive radio in allowing use of licensed spectrum by unlicensed users. Frequency-hopping spread spectrum uses rapidly changing carrier frequency while code-based spread spectrum uses a system of orthogonal codes. In either case, the receiver and transmitter must be accurately synchronized to each other, with an offset being very difficult to recover from without a system reset, which can defeat the purpose of the communicates system in the first place. 


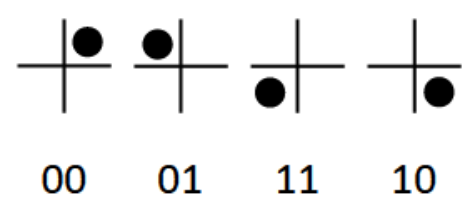

Figure 1.8: Example constellation diagram of a QPSK modulated signal

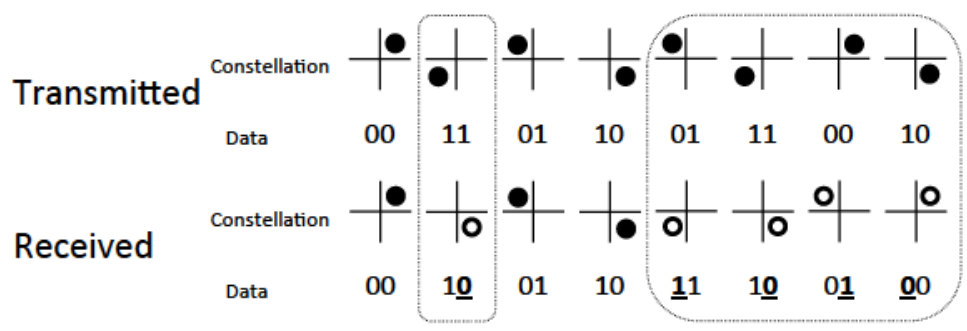

Figure 1.9: Example of the reaction of a QPSK system to a symbol error and a phase offset.

- Phase modulation: Another example can be illustrated by looking at demodulating phase modulated data. For this example, the relatively common modulation format of quadrature phase-shift keying (QPSK) is used, although the issues shown here can be generalized to all phase modulated or quadrature amplitude modulated (QAM). This form of modulation encodes 2 bits per symbol by varying the symbol's phase with 0 degrees being 00, 90 degrees being 01, 180 degrees being 11, and 270 degrees being 10. Grey coding is used to minimize bit errors. The phase of each symbol can also be plotted on a cartesian constellation diagram to allow visualization. Fig. 1.8 shows such a sample constellation diagram of a QPSK system.

Fig. 1.9 shows an example of a QPSK communication system, showing the transmitted and received constellations and corresponding data. To show the effect of symbol errors and phase offsets, the second received symbol is given a 90 degree phase error, as could happen due to thermal noise, phase jitter, cross-talk/interference, distortion, etc. The fourth and subsequent symbols are given a 90 degree phase offset, which could arise from a cycle slip. As is shown, the phase error in the second symbol results in a 1 bit error, while the phase offset results increases the bit error rate to $50 \%$, effectively making the channel useless (since the same result could also be achieved by choosing random numbers). In real communication systems, this would cause a loss in data transfer which would be detected at a higher level in the communication system, eventually resulting in a reset of the system to recover timing. However, this results in loss of data and increased latency until the system is relocked.

One possible solution (which has been used successfully in optical communication sys- 


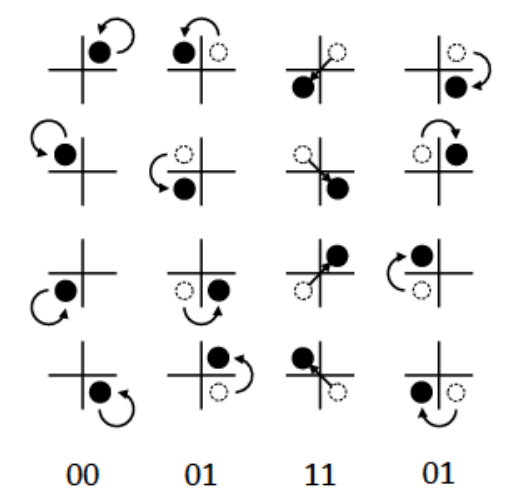

Figure 1.10: Example constellation diagram of a DQPSK modulated signal

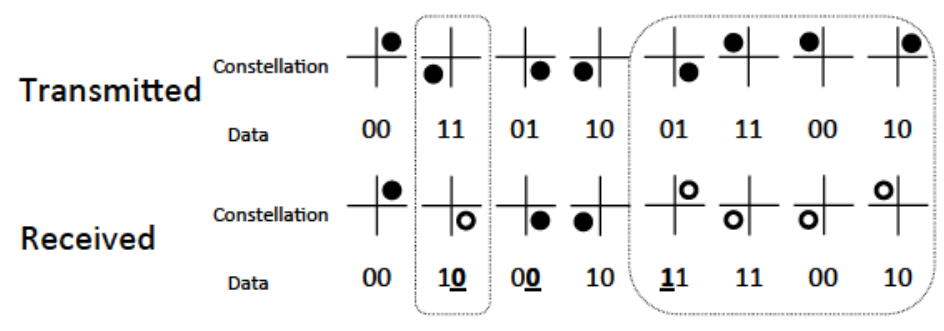

Figure 1.11: Example of the reaction of a DQPSK system to a symbol error and a phase offset.

tems) is the use of differential encoding. Differential QPSK (DQPSK) uses the phase difference between symbols to encode the data, rather than the absolute phase itself. Fig 1.10 shows an example constellation diagram of a DQPSK system. In this example, a phase difference of 0 degrees is 00,90 degrees is 01,180 degrees is 11 and 10 degrees is 10 .

Fig. 1.11 shows a similar example of a communication link with the same impairments as in the first example: a 90 degree phase error on symbol 2, and a 90 degree phase shift affecting symbols 4 to 8 . As can be seen, the phase shift on symbols 4 to 8 result in only a single bit error. However, the cost of this is an increase in the native bit error rate, as can be seen by the fact that the single phase error in symbol 2 resulting in 2 bit errors. Thus, while the risk in avoiding system outages from cycle slips is removed, the bit error rate has increased, which lowers the system's range or bit rate or increases the system's complexity and power consumption. Thus, while the use of differential encoding removes the issue of phase offsets corrupting the communication channel, a solution that prevents the phase offsets in the first place would be better. 


\subsection{Objectives}

These examples have shown some of the problems that phase offsets and cycle slipping has in frequency synthesizers. This thesis examines one of the main causes of such cycle slipping: frequency band switches to compensate for frequency drift or power supply changes, and proposes a solution that can be used to prevent this: a circuit in a critical portion of the VCO that allows these band switches to still be made without cycle slipping and without phase offsets. This circuit uses minimal power and silicon area, and allows integration with existing frequency synthesizers with only slight modifications. It also does not affect the general operation of the phase noise of the circuit or the tuning range of the VCO. The theoretical behaviour of this circuit will be analyzed, followed by the design and measurement of a test chip to demonstrate these concepts. Further improvements in the circuit to reduce variability and to reduce noise will also be shown.

\subsubsection{Summary of Contributions}

The principal contributions of the proposed work are as follows:

- Analysis of a phase locked loop system, demonstrating that stable operation in its linear region can be maintained with a gradual VCO frequency band transition and analysis of conventional VCO frequency band implementations, showing that gradual frequency band transitions can be made to take place with appropriate switch circuitry and control signal generation

- Design and implementation of an appropriate switching circuit that can be used to create gradual frequency band transitions with conventional VCO frequency band circuits that is compatible with legacy layouts, is easily implementable, is stable and predictable over temperature and process corners, uses minimal silicon area or power consumption, and has minimal impact on the PLL during its normal operation. This is in addition to the verification of the complete system in simulation and in measurement, showing transient suppression of $99.3 \%$ and the prevention of cycle slipping

- The modification of the slow switching circuitry to be controllable and stable over process, voltage and temperature with the use of a calibration circuit

- The modification to the control circuitry of the slow switching circuit to allow rise/fall times to be extended arbitrarily 
- Analysis of power supply noise up-conversion in complementary cross coupled VCOs and the development of cancellation circuitry using capacitive and magnetic coupling to prevent or reduce this, reducing the phase noise by $12 \mathrm{~dB}$ (as shown in simulation)

\subsection{Outline}

This thesis is divided into several chapters. Chapter 1 has introduced the main issues and the motivation for this research and chapter 2 will further discuss background into frequency synthesizers, frequency drift and cycle slipping, while showing conventional solutions currently present in the literature and their shortcomings. Chapter 3 presents a concept that solves the issue of cycle slipping without these short comings, an analysis of circuit and its systems behaviour, while chapter 4 and chapter 5 present the design and test respectively of a test chip demonstrating this concept. Chapter 6 discusses further improvements to the circuitry discussed while chapter 7 concludes this thesis. 


\section{Chapter 2}

\section{Background}

The previous chapter has introduced the importance of frequency synthesizers in modern communication systems, and presented the issue of phase offsets, which can cause performance degradation. This chapter will further look at introducing some basic concepts of frequency synthesizers, and will show how frequency drift can cause PLLs to lose lock, requiring the switching of frequency bands and resulting in cycle slipping. Current solutions, as documented by the literature, will be shown, along with their resulting trade-offs.

\subsection{Overview of Frequency Synthesizers}

While there are a variety of different possible implementations of a frequency synthesizer, such as a mixer based topology or direct digital synthesis. The most commonly used circuit is based on a phase-locked loop (PLL). PLL based frequency synthesizers can be integrated almost completely on an integrated circuit (IC) with moderate area requirements, give good output noise with reasonable power consumption, and can have a wide tuning range with small minimum frequency step sizes.

A system level view of a typical PLL frequency synthesizer is shown in Figure 2.1. A reference signal, commonly generated from a crystal oscillator or another stable frequency reference is used as a timing reference. The output of the PLL is taken from voltage controlled oscillator (VCO) although the output may be a divided down version of the VCO for lower frequency ranges. Due to the fact that the VCO typically operates at a much higher frequency than the crystal oscillator, the VCO is fed into a divider first before comparing the timing of the two signals. These two signals are then compared using a phase detector, which gives an error signal proportional to the phase difference between the reference signal and the divided down VCO output. This phase error signal is then filtered and fed back into the VCO, implementing a feed back system that attempts to minimize phase difference between 


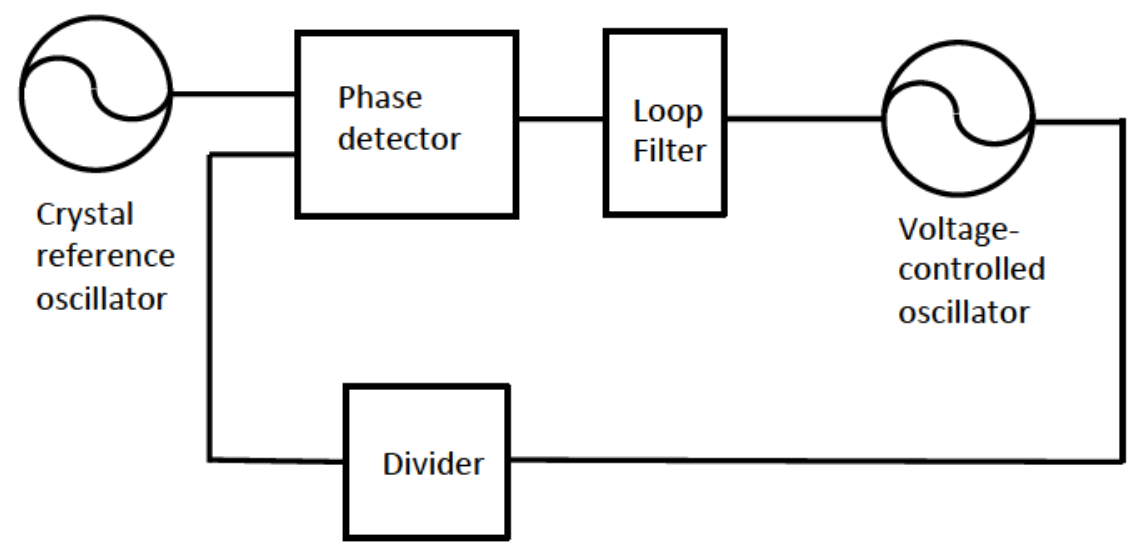

Figure 2.1: System diagram of a typical PLL

the VCO and the reference signal.

If the division ratio $\mathrm{N}$ is variable, then the output of the $\mathrm{VCO}$ is programmable, since each value of $\mathrm{N}$ results in a different output frequency. A common modification is the use of a fractional- $\mathrm{N}$ frequency synthesizer, where the division ratio changes rapidly between different values, implementing an average value that lies somewhere in between two integer values. This allows for a much higher frequency resolution than would otherwise be possible.

\subsubsection{Voltage Controlled Oscillator}

In a frequency synthesizer, one of the major circuits is an oscillator. The oscillator provides the frequency synthesizer's output. In general, many frequency synthesizers use a voltage control oscillator (VCO) as the main oscillator. A VCO consists of an controlled, but unstable circuit whose oscillation frequency is determined by a control voltage. VCOs generally are designed to have low phase noise with minimal power consumption. Other important design considerations include wide tuning range, power supply noise immunity, and high output power.

Oscillators can be created in many different manners, since they only rely on the presence of an unstable circuit. One circuit used low frequency oscillators is the relaxation oscillator, which uses a constant current feeding into a fixed capacitor as a time reference. When the capacitor voltage reaches a particular level, the voltage is reset and the process starts over again. Because the frequency can easily be controlled by adjusting the current, the control over the frequency can be very good, and power consumption can be well controlled with small enough capacitors. Another common circuit used is the ring oscillator, which consists of an odd number of inverting stages connected as a ring. The delay between stages and 
the number of stages controls the output frequency, which can often be controlled by a bias current or voltage, or through the use of a varactor affecting the loading of each stage. A variation on this circuit is the use of transmission lines to implement delays, leading to the distributed topologies such as traveling wave and standing wave oscillators. One of the most common methods for low noise oscillators is the use of an LC tank to act as a resonant circuit. These LC oscillators can exhibit much lower phase noise for the same DC power consumption than other topologies due to their reliance on passive component values for frequency regulation and noise filtering, although this comes at the cost of reduced tuning range and a very heavy dependance on manufacturing parameters that can change from process to process. The tuning range of an LC oscillator is generally controlled using varactors with larger capacitance steps that can be added or removed through the use of fixed capacitances in series with MOS switches.

There are two main types of LC circuits that are commonly used: the differential Colpitts circuit and the Cross-coupled pair, both of which have similar performance as far as tuning range, phase noise and DC power consumption goes. The cross-coupled pair remains heavily favoured in many designs due to its wide tuning range (limited only by the resonator tuning range), intrinsic differential topology, and relaxed start up conditions.

An important property of a VCO is the tuning voltage gain $\mathrm{K}_{\mathrm{VCO}}$, typically expressed in $\mathrm{MHz} / \mathrm{V}$. In order to maintain a large tuning range over a constant maximum voltage range, a large $\mathrm{K}_{\mathrm{VCO}}$ is needed. However, this can worsen the phase noise of a VCO, since small voltage perturbations will generate larger frequency offsets. The tradeoffs between these two will be discussed in more detail later.

Another reason why $\mathrm{K}_{\mathrm{VCO}}$ would need to be decreased would be to facilitate alternate PLL topologies that seek to have high gain components elsewhere in the loop. For instance, the divider-less sub-sampling PLLs presented in $[22,23]$ or the use of a bang-bang phase detector in [24] have very high phase detector gains for the purposes of suppressing charge pump noise. To compensate for these high gain components in order to achieve loop stability, the VCO gain needs to be reduced, thus requiring small $\mathrm{K}_{\mathrm{VCO}}$ values.

\subsubsection{Reference Oscillator}

In general, the frequency of a VCO will drift due to low offset frequency phase noise. One of the main purposes of a PLL is to compensate for this drift by locking it to an oscillator whose frequency is very stable over temperature and manufacturing variations. A common implementation of such a reference oscillator is a crystal oscillator, although alternatives using heavily compensated passives, and MEMS structures are also possible. Crystal oscil- 
lators are well characterized, relatively inexpensive and very reliable, often able to achieve frequency accuracy within $100 \mathrm{ppm}$. Reference oscillators cannot be used for signal generation since they generally have a poor tuning range and are limited in output frequency to below $100 \mathrm{MHz}$.

\subsubsection{Divider}

In most frequency synthesizers, the output frequency is much higher than the reference oscillator frequency, due to the difficulty in creating high frequency, low noise oscillators with extremely stable characteristics and due to the need for a reasonable frequency step size with integer divide ratio steps. Thus, a frequency conversion circuit must be used to divide down the output of the VCO in order for its phase to be compared to the reference oscillator. This is most commonly done using a frequency divider, which essentially uses the output VCO frequency as a clock for a digital state machine whose output is a fraction of the input. Although dividers are commonly viewed as simple circuits, dividers can add jitter and are a major contributor of the DC power consumption of a PLL, since they are one of the highest frequency components of a PLL. Thus, the selection of circuit topology for a divider can play a significant role in the design of the frequency synthesizer. For clock generation and RF frequencies, particularly with high speed CMOS processes, this is often realized using CMOS flip-flop circuits, although at higher frequencies (such as mm-wave), more specialized topologies such as static or dynamic CML or Miller divider may be required.

Frequency synthesizers require a wide output tuning range, which is typically implemented by changing the division ratio $\mathrm{N}$ in the phase-locked loop. In order to implement variable division ratios, a programmable divider that is able to change its division ratio is needed, such as the use of a multi-modulus divider. Fractional division ratios commonly use the same circuitry by dynamically adjusting the division rapidly between multiple values to implement the equivalent of a fractional division ratio.

An important consequence of the use of frequency dividers is their inherent property in reducing any phase shifts inherent in the original signal. As a result, phase noise is reduced at the output of the divider, which limits the ability of the PLL to compensate for VCO phase noise, since the divider acts as an attenuator of the phase noise before the noise is sensed.

\subsubsection{Phase detector and charge pump}

A PLL attempts to correct the difference in phase between the reference and VCO frequency in order to minimize phase errors. In order to do this, a circuit known as a phase detector 


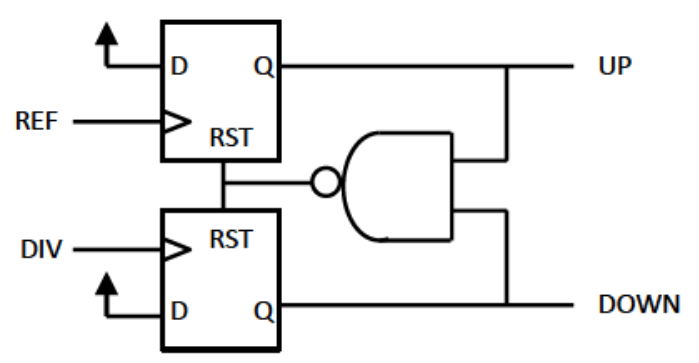

Figure 2.2: Schematic of a typical tri-state phase-frequency detector

is needed to sense the difference in phase between the reference frequency and the divided down VCO output. In ideal system level views of a PLL, the phase detector is commonly viewed as a circuit whose output is the phase error of the two input signals. However, in real implementations, phase detectors commonly work by detecting the edge between low/high transitions between the two signals, working as a sampled data converter which converts the phase difference into a time modulated output. An important limitation of real phase detectors is their general inability to differentiate phases that are integer multiples of 360 degrees apart, leading to non-linear phase characteristics for large phase offsets.

Different versions of phase detectors exist which range from simple logic gates to far more complicated circuits. Important metrics differentiating between different implementations of a phase detector include its response to phase inputs characteristics, sensitivity to small phase differences, frequency range, output jitter, and DC power consumption.

A very common implementation of a phase detector is the tri-state phase-frequency detector, whose schematic can be seen in Fig. 2.2 and whose state diagram can be seen in Fig. 2.3. If the reference signal is faster than the divided down VCO signal, the state machine will tend to switch between the RST and UP states, while if the reference signal is slower, the state machine will tend to switch between the RST and DOWN states. If the reference and divided down signals are the same in frequency, the system will tend to stay mostly in the RST state and occasionally going to the UP or DOWN states, depending on the phase differences between them. Finally, if the signals are identical in frequency and in phase, a race condition will occur, allowing the machine to alternate between the RST and the RP states. Thus, by comparing the UP and DOWN outputs, both phase and frequency differences can be determined. If one signal is faster than the other, the UP or the DOWN outputs will regularly be high while the other will always be low. For phase differences, the difference in pulse widths between UP and DOWN pulses will give the phase difference.

The UP and DOWN outputs can be used by feeding it into a charge pump. A charge pump circuit mainly consists of two current sources that are connected to MOS switches. 


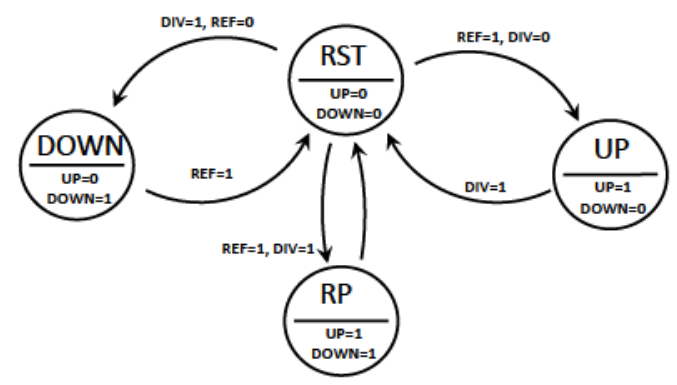

Figure 2.3: Schematic of a typical tri-state phase-frequency detector

Since the charge pump is often connected directly into the control voltage of a VCO (with a filter connected in parallel), the output characteristics can heavily influence the VCO output. For instance, the charge pump output noise is up-converted by the VCO into phase noise, and the periodic nature of a PLL means that imbalances in the charge pump can create output spurs. Thus, many variations have been presented on charge pump circuits to reduce noise and reduce periodic signals that can create spurs. In order to maintain constant loop characteristics across different VCO output levels, the charge pump should also be designed with a high output impedance, although the use of an active filter, dual path filters or other techniques to cause the charge pump output to operate at a constant output voltage is also possible.

\subsubsection{Loop Filter}

An important part of a PLL is the loop filter, which is used to filter out the discrete signals produced by the phase detector and charge pump to create a smooth control signal used to modulate the VCO. Common loop filters consist of a capacitor in series with a resistor (which are used to determine the loop's primary characteristics), which is typically in parallel with a smaller capacitor (which is used to filter out spurs). The loop bandwidth of many PLLs is kept low in order to attenuate spurs and other higher frequency steps, but must be high enough to satisfactorily attenuate the VCO phase noise. Typical loop bandwidths range from $100 \mathrm{kHz}$ to several $\mathrm{MHz}$, and can be implemented using off-chip components or very large on-chip components.

The schematic for a typical passive PLL loop filter for a PLL using a charge pump PFD can be seen in Fig. 2.4. This filter consists of a series RC segment, which determines the primary loop characteristics such as stability, bandwidth, and transient response. A parallel capacitor serves to filter out additional transients that occur due to the sampled nature of the PFD. The final segment is a low pass filter which is used to filter out spurs and other 


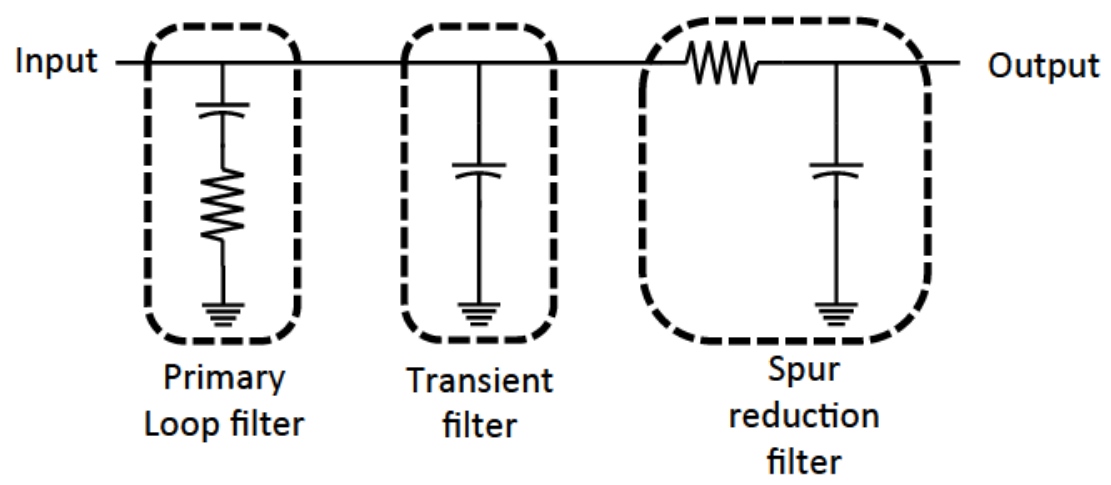

Figure 2.4: Schematic of a typical PLL loop filter

high frequency noise sources. While Fig. 2.4 shows it implemented with a first order RC, circuit, higher order filters are possible, or it may be omitted altogether.

Different configurations are possible, such as the use of active filters for reduced size or for optimizing charge pump linearity by limiting the charge pump output voltage range.

\section{$2.2 \quad$ All-Digital PLLs}

A variation of a PLL that is increasingly becoming more common is the all-digital PLL (ADPLL). In an ADPLL, to as much of a degree as is possible, the PLL topology is kept in the digital domain. This, in theory, allows for several benefits, including being able to leverage in the performance of digital circuits with smaller process nodes, reduced area for filter implementation, lower power dissipation, and easier portability across different process nodes. In reality, while some of these advantages are present (particularly due to the removal of the large capacitors used for the loop filter), ADPLLs still use analog components in the form of a digitally-controlled oscillator (DCO) and a time-to-digital converter, whose analog properties play key determinants of the performance of an ADPLL, making it difficult to realize all of the theoretical advantages that operating in the digital domain has.

While digital PLLs have been proposed and studied for several decades [25], their implementation has changed, as newer technologies enable different topologies. Earlier examples of digital PLLs implemented the DCO using a divided down higher frequency reference clock, using the divide ratio as the digital control $[25,26]$. This limited the maximum PLL frequency, and imposed constraints on the output frequency. Later implementations used variations on ring oscillators [27-31] (which tend to have poorer phase noise performance when compared to LC oscillators with similar power consumption) or using oscillators that 


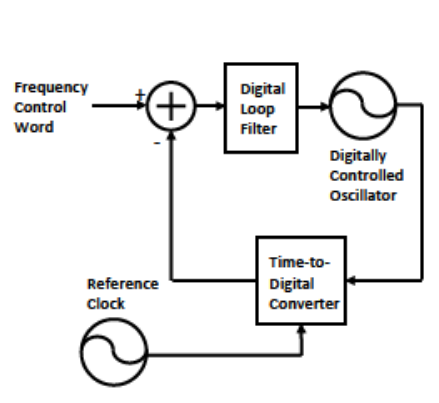

(a)

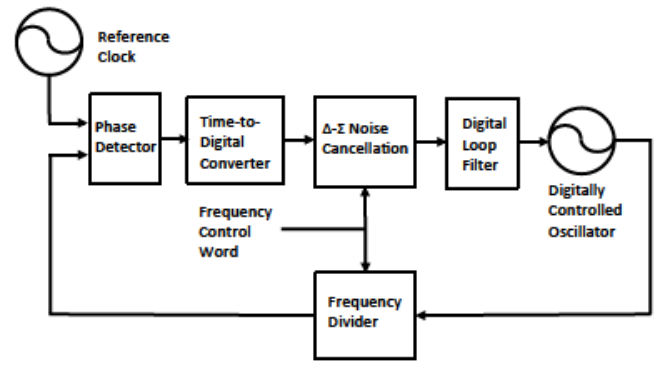

(b)

Figure 2.5: System diagrams of 2 possible configurations of an all-digital PLL

control an analog control port (such as a current or voltage control) with a DAC [32-34] (which negates some of the advantages of a digital topology). More recently, a DCO designed with an intrinsic digital interface has been made possible, as fine geometry CMOS process nodes have allowed for large arrays of small capacitors, which can be used as a fine-tune port for the oscillator [35]. This allows for the low noise performance of an LC oscillator while still maintaining digital control. Hybrid variations also exist, such as [36,37], which use an array of many small varactors, each controlled by DACs for fine tuning control. In addition, [36] demonstrates a method of preventing cycle slipping when switching between reference clocks with different phases by measuring the phase offset between the two reference clocks and applying a DC correction offset when switching from one to another. While this has similar goals to the research shown in this thesis (the prevention of cycle slipping while conducting a major change in the PLL's operating conditions), there are also significant differences as well, since [36] involves switching reference clocks rather than VCO frequency bands, and does so with digital offset correction to minimize the effective error seen when performing the switch, rather than applying the switched stimulus gradually. Still, this serves to provide partial validation of the approach shown in this thesis by showing how a PLL can be perturbed without it leaving its linear region if the perturbation is adequately compensated for.

Currently, a common implementation of an ADPLL can be seen in Fig. 2.5(a), which has a TDC replace the divider, phase detector, and charge pump in a PLL, and the DCO replace the VCO. The loop filter is implemented digitally using digital signal processing (DSP), and the reference signal is used to clock the circuitry. The TDC measures the DCO output phase relative to the reference phase to determine the frequency/phase offset. The error signal is processed through the loop filter, and is used to generate the control signal to the DCO.

An alternate implementation can be seen in Fig. 2.5(b), which uses a divider (similar to 
a conventional PLL) and uses the TDC between the reference signal and the divided down DCO output. This has the advantage that the TDC input is at a much lower frequency, which reduces the design constraints, but is susceptible to spurs in a fractional-N loop, since the TDC sees the phase after dithered division ratio. This can be countered by using a noise cancelation circuit which applies phase offsets to the TDC value, since the division ratio is always known, so the necessary offset can always be calculated.

Similar to this is the use of an conventional phase detector and charge pump with an ADC to digitize the timing offset of the divided down DCO signal to the reference [38,39].

A TDC is typically built using a large series of buffers to generate a delay line [34], with the number of buffer stages needed being determined by size of the phase difference between the DCO/reference signals. A major problem with TDC design is designing a circuit sensitive enough to very small time variations while still maintaining a large enough dynamic range to measure large time offsets. Many variations exist on the basic delay line circuit to solve these issues, including parallel delay lines with unequal delays, known as a Vernier delay line, which reduce the minimum time step to the order of delay matching rather than the absolute delay [40], a combination of conventional and Vernier delay lines to create coarse/fine TDC using fewer stages than would be required using Vernier delay lines [41], 2 dimensional delay lines, which allows for larger time steps to be measured without a linear increase in the number of delay stages [42], multiple parallel delay stages to reduce the number of delay stages [43], and local passive interpolation, which use passive components to generate interpolated signals to increase resolution [44]. Ring oscillators can be used as an indefinitely long delay line to increase the sensing range and introduce noise shaping either with delay stages $[30,37,45,46]$, or Vernier delay lines [47]. Ring oscillators can also be used in pulse shrinking topologies, which have been shown to achieve fine time resolution without calibration circuitry [48]. Time amplification circuits can also be used to increase small delays to larger values using metastable circuits to allow for detection of delays that would otherwise be too small to measure $[49,50]$.

In a DCO, the oscillation frequency is controlled by a digital control signal. Typical DCOs are implemented in a similar manner as a VCO, but with large banks of switched capacitors or varactors to allow the frequency to be digitally controlled. A major issue with DCO design is obtaining small enough frequency steps while maintaining tuning range. In general, discrete MIM capacitors and varactors cannot be made small enough to achieve adequate frequency steps on their own, so other methods to reduce frequency step sizes are needed. This includes using small varactors connected to a digitally controlled, analog voltage, dithering a control signal with a high speed $\Delta$ - $\Sigma$ modulator [35,39], using impedance dividing circuits [38, 39, 51,52], or EM structures using artificial dielectric lines [53]. 


\subsection{General PLL Operation}

The previous sections have discussed the various components of a PLL. This section will briefly discuss how these components work together to implement a PLL that can be used for frequency synthesis in order to give the proposed contributions context. More details about this can be found in many standard references [54-56].

\subsubsection{Start-up}

When the PLL is first started, the divided down VCO is at a different frequency than the reference signal and is at a difference phase with it. The purpose of the loop is to attempt to correct both of those issues. This is done mainly through the use of the phase detector and the loop filter, with the phase detector measuring the phase offset and loop filter attempting to shift the VCO control signal to stabilize this offset.

Frequency acquisition is one of the first goals of the PLL. This can happen in different ways, depending on the PLL implementation. When the frequencies of the reference and the divided down VCO differ, the time difference between successive rising or falling edges will vary rapidly, with the reference leading the VCO half of the time and vice versa. Thus, PLLs using a simple phase detector based on a binary gate (such as an XOR gate) will have trouble locking the frequency since it does not generate enough information to determine which signal is faster than the other, although it should be noted that this is not quite true for the specific case where the frequencies are very close to each other, since time difference between successive edges will not vary as rapidly, and the loop may be able to lock the VCO frequency before leading/lagging signal changes places. For these types of PLLs, an alternate circuit is required to lock the frequency first before phase lock can be acheived. Many PLLs use a tri-state phase detector with a charge pump. This circuit uses a state machine and can retain information about previous states, giving it the ability to determine if the divided down VCO signal is faster or slower than the reference signal, allowing it to achieve frequency lock on its own.

Once frequency acquisition has been achieved, the PLL will then try to achieve phase lock. For loops with an integrator in the loop filter, the integrator will accumulate any offsets of phase, so when the loop settles, there will be no phase offset between the two signals. Otherwise, some phase offset will remain. Once the phase offset is low enough, the system is considered to be locked, and the PLL enters its steady state operation. 


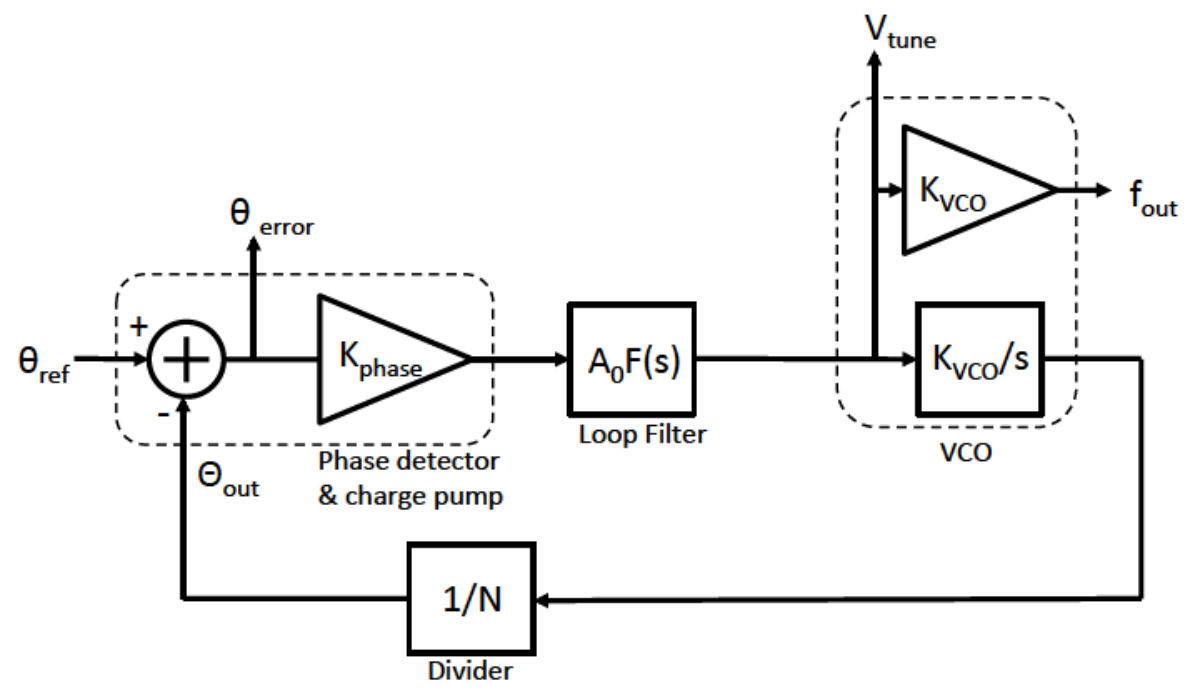

Figure 2.6: Laplace system diagram of a PLL

\subsubsection{Locked Operation}

During locked operation, both the reference and divided down VCO signals are at the same frequency. Due to phase noise or other disturbances, small phase offsets may be introduced. To understand how the PLL is able to compensate for these, the PLL is modelled in the Laplace domain. A typical PLL system diagram is seen in Fig. 2.6. Here, the phase detector is shown as a adder with gain $K_{\text {phase }}$, the loop filter is a generic block with transfer function $A_{0} F(s)$, the $\mathrm{VCO}$ is treated as an integrator (since the control signal affects its frequency which is the derivative of its output phase) with a gain $K_{V C O}$, and the divider is treated as a $1 / N$ attenuation block.

From this diagram, the closed loop transfer function of the system can be determined. While this expression is given in terms of $\theta_{\text {ref }}$, this transfer function can be made to reference any value in the system by multiplying by the appropriate blocks.

$$
\frac{\theta_{\text {error }}}{\theta_{\text {ref }}}=\frac{s}{s+\frac{K_{\text {phase }} A_{0} K_{V C O}}{N} F(s)}
$$

Many PLLs use a tri-state phase detector with a charge pump, and a filter which can be approximated as $\frac{s+\omega}{s}$, whereby this expression takes the form of

$$
\frac{\theta_{\text {error }}}{\theta_{\text {ref }}}=\frac{s^{2}}{s^{2}+\frac{K_{\text {phase }} A_{0} K_{V C O}}{N} s+\frac{K_{\text {phase }} A_{0} K_{V C O}}{N} \omega}
$$

This then shows that the PLL operates as a high-pass second order system with loop 
parameters that can be controlled by the loop gain and the filter coefficients. Note that while this transfer function is relative to the reference phase, a similar expression can be generated relative to any phase offsets added by the VCO (which would include phase noise). The bandwidth of the loop is largely controlled by the corner frequency of the filter. By choosing proper coefficients, the damping constant can be made from 0.5-1, allowing rapid recovery from any errors. Due to the nature of linear systems, larger offsets result in larger correction factors, resulting in exponential decay of the offset (or alternatively, correcting the offset in logarithmic time).

Note that in order to attenuate some of the noise that is present due to the fact that a PLL is actually a sampled time circuit rather than a continuous time circuit, additional filtering may be added. Since this is typically done well above the loop bandwidth, this does not play a strong role in the loop dynamics.

\subsubsection{Cycle slipping}

As discussed in the previous section, the PLL has a negative feedback path which allows for correction of small and even moderate phase offsets. Due to the linear nature of the components involved, larger phase errors create larger voltage offsets which cause larger correction factors to be implemented. Since all of the factors scale together, phase errors of any size will be corrected relatively quickly.

However, in real implementations, such a linear response can only be achieved for a limited size of phase errors. Since the reference frequency and the VCO output are both cyclical signals, it is difficult to differentiate between offsets if they are an integer multiple of reference periods apart. Charge pump phase detectors are able to alleviate that, particularly those with many states, but still add a hard limit to the maximum linear region.

If a phase error ever exceeds this linear limit, the linear region expressions no longer applies, and the loop essentially behaves as if it were no longer frequency locked. As such, the phases of the divided down VCO and reference will slip past each other, hence the term cycle slipping. In charge pump PLLs, recovery from this can occur, but takes a longer time since the linear loop response no longer applies. Instead, the phase recovery time is determined by the ratio of the charge pump current and the loop filter capacitance, leading to a much slower response time that increases linearly with the phase offset. Once the frequency and phase are recovered closely enough, the loop once again enters its linear region, and quickly regains lock.

In addition to much longer settling times, this also has the effect of introducing a phase offset, since the reference edge that was originally locked to by the divided down VCO will 
be different from the edge it will be locked to after the cycle slipping behaviour ends. As described earlier, this can have serious effects on the system the PLL is used for, depending on application.

Cycle slipping occurs when large phase offsets are introduced into the loop. While in theory, VCO phase noise could cause cycle slips due to its random nature, although statistically speaking, this is highly unlikely. More probable is external interference, such as EM interference, or coupling to other portions of the system such as power amplifiers or high power clock distribution networks, although the risk of this can be mitigated through careful design and floor-planning. A more likely scenario causing this is when the VCO band is switched. This causes an near-instantaneous frequency shift, which will almost certainly cause the PLL to lose phase lock. For systems requiring constant lock of a PLL, such band switches are highly undesirable, and would only occur if necessary, such as the locked frequency falling out of the VCO band's range due to temperature drift.

\subsection{VCO Frequency Drift}

Temperature changes can affect many components in a circuit. In many circuits, changes in temperature results in slight variations in semiconductor parameters, which can affect optimal bias points and noise/gain characteristics of a transistor. However, with a VCO, temperature changes also affect the tuning range and frequency of the oscillator. Frequency variations due to temperature variations can arise from many different sources, such as temperature coefficients for passive components [57] and dependance on temperature of semi-conductor parameters of active materials [58]. Even variations in inductor temperature can vary the amount that skin effect plays, changing the resonant frequency of an LC tank since the inductor resistance creates a slight shift in resonant frequency [59]. Changes in physical dimension due to thermal expansion is also possible, although this plays less of a role than the other factors [60].

Temperature compensation in frequency synthesizers is increasingly becoming an important design issue. This can be demonstrated through a simplified model of the output frequency of a VCO:

$$
f_{V C O}=f_{0}+K_{K V O} \Delta V+K_{T E M P} \Delta T
$$

where $f_{\mathrm{VCO}}$ is the $\mathrm{VCO}$ output frequency, $\mathrm{f}_{0}$ is the frequency at a particular tuning voltage and temperature, $\Delta \mathrm{V}$ is a tuning voltage offset, $\mathrm{K}_{\text {TEMP }}$ is the oscillator temperature sensitivity and $\Delta \mathrm{T}$ is a temperature offset. To maintain a constant frequency in the presence 


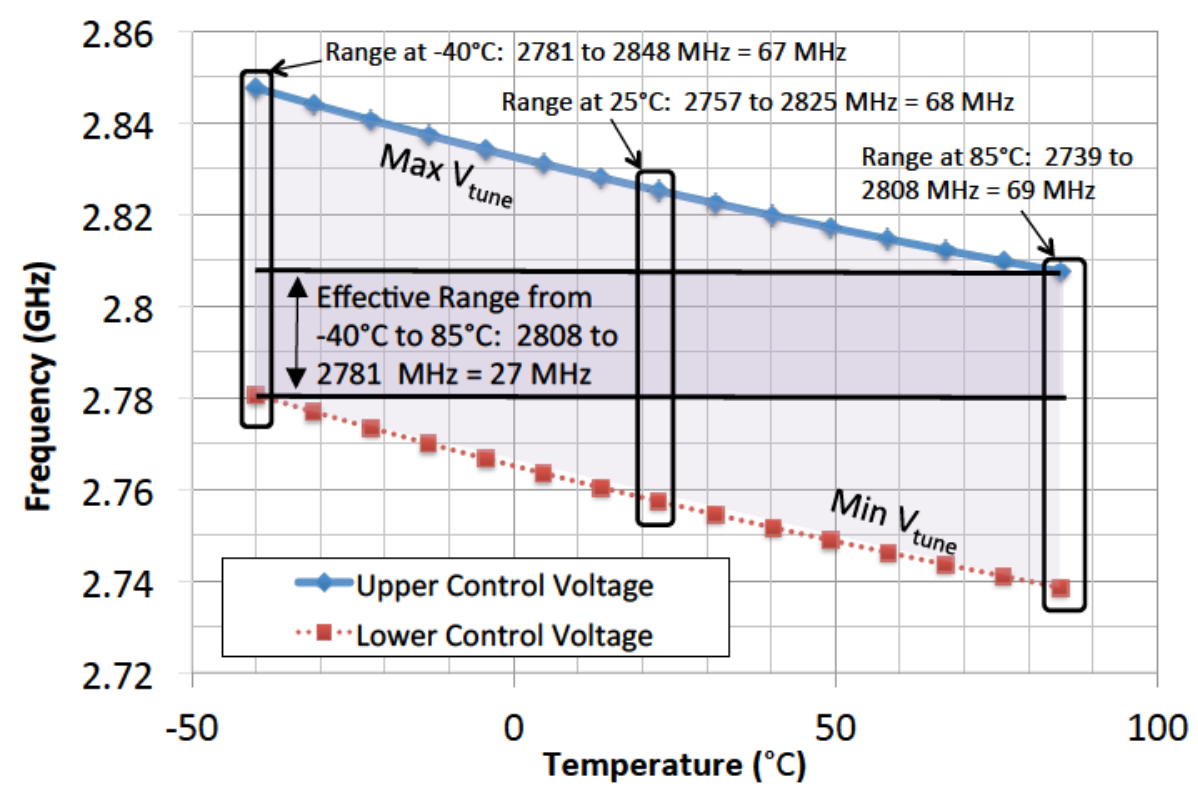

Figure 2.7: Plot of the simulated upper and lower tuning voltage of a frequency band of a VCO over temperature

of a temperature shift $\Delta \mathrm{T}$, the PLL must apply a control voltage shift:

$$
\Delta V=-\frac{K_{T E M P}}{K_{V C O}} \Delta T
$$

As described previously, it is often desirable to reduce $\mathrm{K}_{\mathrm{VCO}}$ by reducing the size of the tuning varactors in order to reduce the VCO phase noise. However, as $\mathrm{K}_{\mathrm{TEMP}}$ arises not just from the tuning varactors, $\mathrm{K}_{\mathrm{TEMP}}$ does not change as quickly as $\mathrm{K}_{\mathrm{VCO}}$. Thus, an unwanted consequence to decreasing $\mathrm{K}_{\mathrm{VCO}}$ is the increased value of $\Delta \mathrm{V}$ required to maintain a stable frequency. This can also be illustrated graphically as in Figure 2.7, which shows the output frequency of an oscillator at the maximum and minimum tuning voltage for a single frequency band over a temperature range of $-40^{\circ} \mathrm{C}$ to $85^{\circ} \mathrm{C}$. While the frequency range of the band is more than $67 \mathrm{MHz}$ at any given temperature, the range of frequencies covered over the entire temperature range is only $30 \mathrm{MHz}(2.78 \mathrm{GHz}$ to $2.81 \mathrm{GHz})$, a reduction by more than $50 \%$. Thus, the temperature coefficients of the circuit components have a strong effect on the output frequency. If $\mathrm{K}_{\mathrm{VCO}}$ is reduced, then the two lines move closer to each other, further reducing the overlapping region. Past a certain point, the frequency band no longer provides coverage of any single frequency over the entire temperature range. A similar effect also occurs as supply voltages are reduced (due to process scaling).

With a significant enough temperature change, the PLL will attempt to compensate by adjusting the tuning voltage, but will reach the supply voltage or ground before achieving the 
necessary offset. Thus, the necessary offset voltage will not be generated and the PLL will lose lock. As most VCOs have numerous banks of capacitors to achieve numerous frequency bands, the PLL can regain lock by switching to a different band. However, during this process, the PLL remains unlocked at an incorrect frequency, which can adversely affect the performance of the system. With large enough frequency steps, the PLL will enter cycle slipping, a non-linear region of behaviour which can take a long time to move out of.

In theory, DCOs are not susceptible to this problem since the frequency control of a DCO is implemented using a single set of control lines. If these lines are implemented using thermometer coded capacitor banks, continuous, monotonic tuning is assured and temperature compensation can be done by adjusting the DCO code at will without any side effects. In reality, this is not feasible due to the fact this would require a large number of capacitors to achieve frequency resolution of 12-bits or higher, leading to very high parasitic capacitance. Thus, coarse tuning capacitors are frequently used, which play the same role that capacitor banks play in VCOs, and thus, this can cause the same problems with tuning range that exists with VCOs.

There have been many proposed solutions to the issue of temperature drift, which range from removing the temperature sensitivity as much as possible, to the use of auxiliary tuning loops to provide external compensation. Further discussion of existing methods will be the topic of the next chapter. This thesis discusses an alternative solution which overcomes many of the issues inherent in the existing literature, which will be discussed in subsequent chapters.

\subsection{VCO Temperature stabilization}

One of the most frequent solutions is to remove the frequency variations entirely by making the VCO frequency independent of temperature. By preventing the oscillator frequency from changing over temperature, the constraints on the frequency coverage of the VCO becomes considerably relaxed.

Eliminating the frequency dependance on temperature can be done in many different ways. One possible solution is to emulate the frequency stability of crystal reference oscillators, which rely on precisely cut quartz crystals as a resonator to gain a very stable frequency characteristics over a range of temperatures. Examples of this can be seen in [61], but other methods are possible as well, such as Surface Acoustic Wave (SAW) resonators with very good temperature stability needing only minor correction circuitry at most [62-65]. In order to allow for on-chip implementation, MEMS resonators have also been proposed for replacement for crystal oscillators [66-74]. A design based on the thermal characteristics of 
silicon has also been proposed [75]. This method has severe drawbacks in terms of cost, and limitations on operating frequency and tuning range.

A similar strategy is to use thermally stable materials for the construction of resonant structures [76]. However, these methods are meant as implementation of frequency references and are generally not suitable for use as the frequency variable oscillator in a frequency synthesizer, since the stability over temperature is generally achieved by reducing tuning range, or by removing the ability to tune frequency completely.

There are other ways to remove frequency dependance on temperature, ranging from reliance on ratio based circuits such as current mirrors which can be controlled by a band gap generator or other temperature insensitive circuits. This can be seen in the ring oscillators in [77-79] which rely heavily on stable current sources in order to stabilize output frequency. A similar example is shown in [80], which uses a temperature-stable bias current in addition to an amplitude limiter to control the frequency drift of a relaxation oscillator. In [81-83], a particular bias point in the oscillator is found to be more stable against temperature drift. In [84], a band-gap generated current is used to obtain more stable I-V characteristics from a MOS transistor, which is used in a PLL. In general, though, this does not apply to most oscillator topologies, which may offer key advantages that are necessary to the system (lower phase noise, higher frequency, etc.) and more complex circuits are required for proper compensation.

An alternative possibility for temperature stabilization is the control of the actual temperature itself. In oven-controlled crystal oscillators, the oscillating crystal is placed in a sealed enclosure whose temperature is carefully regulated, allowing very low temperature drift as the ambient temperature has minimal effects on the temperature of the resonator. This has also been proposed for MEMS resonators using MEMS heating elements [85].

Other variations have been proposed which involve sensing the effects of variations on temperature or process and changing a control voltage to compensate for this change. In [86], a MOS diode chain is designed which is used to generate a temperature and process varying control voltage in such a way as to compensate for the temperature variations in the ring oscillator delay cells. A similar concept can be seen in [83,87-98]. Examples of mm-wave designs using a variation on this technique can be found in [99-101].

One possible option for ring oscillators is to compensate for temperature variations by comparing the delay of cells with different loads, and inferring what timing offset is needed to account for temperature variations, as was done in [102]. A similar solution is presented in [103], which uses voltage controlled resistors that are controlled by a switching and bias network that is connected to a band gap generator to compensate for temperature variations.

In [104], a circuit designed to compensate for power supply noise also reduces the sensi- 
tivity of the circuit to temperature variations.

In [105], VCO thermal drift is compensated by locating the VCO in a thermally stable area of the chip, a solution which may not be practical in many situations.

In [70], multiple oscillators are used, with one being selected based on the current temperature.

In [106], a built-in self-test (BIST) circuit, consisting of a ring oscillator and a frequency sensor, is used to test for process and temperature corners, using that information to calibrate an LC VCO. A similar approach is presented in [107], where a calibration routine is used to detect for variations in NMOS and PMOS device performance and compensate for them appropriately. In [108], a temperature sensor is used to determine the voltage bias to compensate a crystal oscillator, based on the filtering property of a separate crystal. A temperature sensor is also used in [109] with a digital look-up table to determine the compensation control voltage needed.

In [110], a compensation loop circuit is added to a relaxation oscillator to compensate for delay variations in the comparator due to temperature variation. An alternate approach to solve this is shown in [111] which uses a switched capacitor circuit to compensate for the variation in comparator delay.

One possible solution is to accept the reduced tuning range of each frequency sub-band, as in [112]. Doing so requires that the sub-bands be placed closer together, requiring more sub-bands to cover a larger tuning range, which will add to the parasitics. This solution places a limit on the minimum $\mathrm{K}_{\mathrm{VCO}}$ possible, since an overlap of tuning range during the hottest and coldest operating temperatures is needed, and this larger KVCO will result in increased phase noise. Accurate characterization is needed to determine the actual limits of each sub-band, with process variation needing to be taken into account. In addition, a more complicated sub-band selection algorithm is needed to determine the correct sub-band to use during initial locking, taking into account the current temperature and the limited tuning range. A similar solution is discussed in [60], where initial band selection is done with VCO control voltage set to a fixed value set using a CTAT voltage to help ensure that the band selected is capable of supporting the desired frequency over the operating temperature range.

\subsection{Band gap controlled tuning}

Another possible solution is the use of a secondary tuning port to vary the VCO tuning. Controlling this secondary tuning port can be done with the PLL, as will be detailed in the following section, but a simpler alternative is to control it with a voltage generated by 
a band-gap generator such as a PTAT or CTAT voltage, depending on the temperature coefficients. This has been proposed in [57-59, 105, 113-117]. A similar method, using higher order polynomial interpolation has been proposed in [62]. These methods have the advantage of minimizing system complexity and reducing problems with system stability but can add noise, since the band-gap noise is directly up-converted as VCO phase noise. Another disadvantage is the increase in design complexity, due to the need to ensure that the proportional-to-absolute-temperature (PTAT) voltage that is usually used to compensate for temperature changes exactly compensates for tuning range changes. Thus, this method relies heavily on accurate temperature coefficients in all device and passive models, and is potentially susceptible to process variations.

A variation of this method, using an inversely proportional-to-absolute-temperature (IPTAT) current is shown in [118]

In most cases, frequency synthesizers do not require complete temperature compensation as the feedback loop can compensate for minor errors. Thus, the frequency compensation method can be digitized to reduce the up conversion of noise from the band gap generator. Such an example can be seen in [119], where a PTAT voltage is used to determine the current biasing for the oscillator to control for temperature variations. Since this is done with fully switched transistors, noise from the band gap is blocked. An alternative strategy is proposed in [60], where different sized varactors are switched in to connect them to the tuning voltage depending on the temperature, based on a CTAT voltage. In [120], the MOS transistor threshold voltage and several voltages with variable temperature variabilities are detected and used to determine the resistive loading in a ring oscillator. However, as with directly tuning the VCO with a band gap generated voltage, these strategies still require significant, accurate characterization of all components of the oscillator, and this approach potentially biases the oscillator in a sub-optimal manner, since it is biased for temperature stability rather than for lower noise or lower power consumption.

\subsection{Dual-Path PLL}

Another common solution to resolve the issue of limited tuning range in VCO is the dual-path PLL. In a dual-path PLL, the loop filter is split into different portions, with each portion driven by separate charge pumps and either combined with an adder circuit or with separate tuning ports in the VCO. Dual-path PLLs have been proposed for other reasons such as to reduce the area required for the loop filter as in [121-126], for maintaining a constant PLL bandwidth across the tuning range, as in [127], for reducing susceptibility to power supply noise [128], for reducing delay in the filter path and for having fine control over the 
gain of both paths [129], or for simple control over loop characteristics [130], but have been demonstrated to also be useful to compensate for tuning range variations due to temperature changes, as it essentially adds an additional tuning port onto the VCO to compensate for changes in tuning ranges.

An earlier approach similar to a dual-path PLL is found in [131], where the secondary VCO control voltage is controlled by the control voltage of a dummy VCO that is locked in another PLL.

A variation of this approach for a mm-wave injection locked oscillator is shown in [132], where the tuning range drift due to temperature range drift is inferred by observing the phase difference between the output and the injected signal, requiring a large number of circuit components to detect this phase offset. In [133], a feedback loop based on a frequency sensor is used to control the compensating tuning port of the VCO. In [134], a temperature sensitive bias voltage generated using a resistor is used to control the tuning port.

In [135-137], the secondary VCO tuning is implemented using large varactors. However, very large off-chip capacitors are needed to keep the secondary feedback bandwidth low for stability and to reduce the phase noise added. A similar approach is shown in [138-140] with large low pass RC filters used for the secondary loop, although [139] uses a switched capacitor filter in addition to the RC filter to improve locking time. An alternative to the use of off-chip capacitors is shown in [141] using gm-C cell integrators as part of the low pass filter. In [142] the gm-C cell integrator uses sub-threshold currents to charge and discharge the filtering capacitor very slowly with a source follower with low output impedance to provide a low impedance connection to the large tuning varactor. Another alternative is the implementation of the secondary loop with a digital-to-analog converter connected to the tuning port [143-147].

This approach can be modified by reducing the size of the coarse tuning varactor and using multiple banks while keeping the coarse tuning large with enough range to compensate for temperature variations, as in [148], while an alternative variation is to combine a coarse tuning varactor with switched capacitor banks [149-152].

In [153-157], the secondary tuning is done with arrays of fully switched, small varactors to allow for the use of digital filters. In this way, this approach is similar to what will be presented in this thesis, since these solutions use existing capacitor banks or varactor banks as a way of implement a large tuning capacitor. However, these approaches differ in that they intend for long term operation of the PLL within the transitionary regions (potentially sacrificing performance), and the also differ in the method used to implement the soft capacitor bank switching requiring circuitry that requires more silicon area and DC power. The varactors are either quickly switched, as in [153], or gradually switched using a 
digital-to-analog converter (DAC) with a switch matrix, as in $[154,155,157,158]$. However, large numbers of varactors can increase parasitic capacitance. potentially degrading the tank $\mathrm{Q}$ factor leading to degradation in phase noise, and the DAC and switching matrix require significant silicon area. To avoid noise, the DAC will require a large number of control bits, as shown by the 20 bit DAC in [155] which is followed by an analog reconstruction filter, or the 22-bit $\Sigma$ - $\Delta$ MASH modulator in [157].

In [156], the large secondary capacitance is implemented with an array of switched MIM capacitors using a delta-sigma modulator. However, this comes at the cost of significant area consumption for the capacitor array, and parasitics which reduce the tuning range of the circuit.

In [159], the VCO temperature control tuning voltage is controlled by a digital circuit that measures for temperature changes using a time-to-voltage converter by measuring the time to charge a capacitor with a band-gap generated current. A similar approach is shown in [160], where a high accuracy temperature to digital converter using a MEMS thermistor is used to adjust the frequency of the VCO to obtain temperature stability.

\subsection{Frequency Synthesizer Calibration Circuits}

Automatic frequency calibration circuits (AFC) attempt to resolve this issue by quickly determining which of the VCO frequency sub-bands to use to lock to the correct frequency. These circuits can use many different methods in order to determine whether or not the VCO is operating in the correct band. One possible method is to monitor the tuning voltage of the VCO directly [161-164]. Upon startup, if the VCO is not in the correct band, the PLL will attempt to lock the PLL by moving the control voltage until it reaches either the minimum or maximum voltage. A tuning voltage monitoring circuit for calibration automatically can then switch the VCO bands as the tuning voltage approaches either too high or too low a value. This can also be used to avoid sub-optimal regions of charge pump operation, and does not require a special calibration mode for the PLL, but has a disadvantage of being relatively slow to achieve lock since the calibration circuit needs to spend enough time in each band to attempt to lock. A faster approach can be done by opening the PLL loop by connecting the VCO input control voltage to a fixed value and running a special calibration routine to compare the speed to the reference and the divided down VCO to determine if the selected band is too high or too low. The benefit of this is faster determination of an incorrect band since there is no need to wait for the PLL to attempt to lock. One method of doing this is to count the number of cycles from the reference oscillator and the divided down VCO in a set amount of time, as can be seen in [122,165-169]. An alternate method is to measure 
the actual frequency into a voltage level, such as the circuit shown in $[170,171]$, which uses a time-to-voltage converter to convert the frequency difference between the reference and divided down VCO output into a voltage level that can be used to determine if the band is too high or too low, or the circuit shown in [172], which uses a counter fed directly by the VCO output to determine the VCO band center frequency at different bands. Once it is determined that an incorrect band is being selected, an alternate band is then selected, until it is determined that the correct band is selected. If the number of bands is small (less than 16), the band selection circuitry will typically be able to step through different bands linearly

one at a time, as is done in [161-165,167,170]. With a larger number of bands, selecting the correct band can be done faster using a binary search algorithm, which eliminates half of the remaining bands with each trial, and can converge to the correct band more quickly, if the number of available bands is large. This can be seen in $[166,168,169,171,172]$

\subsection{Summary of Background}

This chapter has discussed background information relevant to proposed band switching circuit. While other solutions have been presented in the literature to avoid cycle slipping, such as the use of dual tuning ports, temperature invariant frequency control, and temperature compensation loops, these invariably have drawbacks of some form or another, possibly with higher design complexity, and/or potential performance tradeoffs in order to prevent a rare, but potentially catastrophic incident. In the upcoming chapter, the proposed circuit, which has minimal implementation requirements and performance tradeoffs while still preventing cycle slipping, is discussed. 


\section{Chapter 3}

\section{Design}

The previous chapters have shown how temperature drifts or power supply voltage changes can cause cycle slipping in frequency synthesizers, which can cause problems for the resulting system they are in. While many solutions exist to prevent cycle slipping, they have drawbacks, such as the limited ability to optimize for phase noise or power consumption, the need for additional tuning ports which can increase phase noise, increased parasitic capacitance which can degrade performance and tuning range, and the need for additional specialized monitoring and control circuitry, with additional design overhead and increased area and power consumption. In this chapter, an alternative will be presented that can resolve this issue by preventing cycle slipping during frequency band switching through a slight modification in the band switching circuitry itself to allow it to switch frequency bands slowly enough for the loop to compensate. This circuit can be integrated in existing designs relatively easily, and has minimal power and area requirements, while not affecting the general operation of the frequency synthesizer's phase noise or tuning range. The resulting tradeoff is a slight increase in phase noise, but only during the relatively infrequent frequency band switches.

The first section will deal with the theoretical basis behind the proposed circuit, while the subsequent section discusses the test chip used to implement this.

\subsection{Analysis of capacitor bank slow switching circuit}

\subsubsection{Capacitor Bank Analysis}

This thesis looks at the use of a slowly switched capacitor bank to obtain gradual frequency transitions. This section will look at the analysis of a typical capacitor bank circuit to create models of how such a circuit will behave, showing that a sufficiently slowly switched capacitor 
bank is able to provide the needed monotonic and continuous impedance shift. Analysis of the effect that operation in the transitionary region has on the quality factor of the tank circuit is also done.

Capacitor bank circuits are generally incorporated in LC oscillators to increase the frequency range without increasing the varactor size, which would increase phase noise and alter the loop gain $[115,173,174]$. In addition, varactors are generally limited in impedance range, relative to their parasitic capacitance. However, depending on the process, varactors may have a higher quality factor than capacitor banks for similar ranges in variable impedances, and may have less variability from process variation.

Switched inductor topologies are also possible but can be problematic at RF frequencies since the inductor impedance $j \omega L$ is relatively low compared to the switch resistance, which would greatly reduce the tank quality factor [175]. At higher frequencies, this effect is lessened for inductors (but becomes worse for switched capacitors), and thus can be effective [176].

Transformer topologies with switched active devices can be used [177-180], but require custom designed electromagnetic structures that need to be characterized very accurately. Switches can also be placed on the inductor shielding to vary inductance [181,182]. The use of multiple VCO cores (each with their own inductor) is a common approach [173] that has better performance than using switchable components in the oscillator tank, but requires more area to implement.

While capacitor banks have been studied in the literature [183], what follows is a set of analyses derived from first principles intended to highlight particular properties of a capacitor bank circuit.

A typical set of capacitor bank circuits can be seen in Fig. 3.1. In all cases, the capacitor bank is connected directly to the tank voltage. A fixed capacitor and/or varactor (as in [184]) is placed in series with a MOS transistor that can either connect the capacitor bank to ground, or to another capacitor. The main limiting factor in using a transistor as a switch is its series resistance (when the switch is turned on) and its parasitic capacitance (which dominates when the switch is turned off). Assuming a fixed gate length, adjusting the width of the transistor allows these two to be traded off, as wider transistors have lower series resistance, but greater parasitic capacitance. The product of these two, $R_{s w}, C_{s w}$, can thus be used as a figure of merit for a transistor switch, as it is independent of transistor gate width.

Theoretically, both the (a) and (b) versions of Fig. 3.1 offer an equivalent figure of merit, since the doubled series resistance in (a) (since two transistors are in the series differential path) is countered by half of the parasitic capacitance (since the parasitic capacitances are 


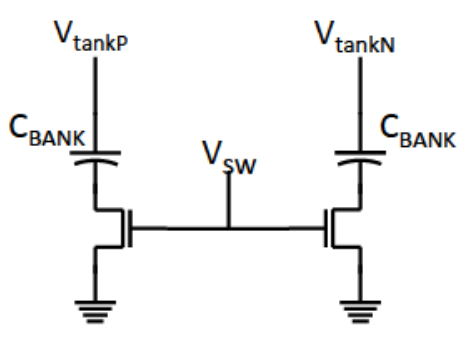

(a)

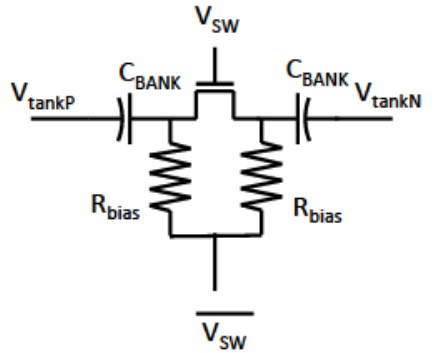

(b)

Figure 3.1: Schematic showing typical VCO capacitor bank circuits

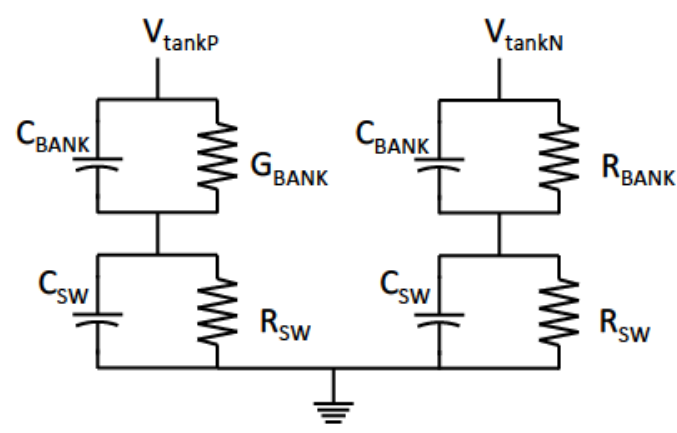

Figure 3.2: Simplified model of a capacitor bank circuit

placed in series). Thus, the selection of one topology over another depends on other factors, such as the fact that the capacitor banks connected to the $\mathrm{P}$ and $\mathrm{N}$ nodes in (a) can be placed away from each other, the fact that (a) requires half of the number of control lines, or the slightly improved ability to turn the transistor off in (b), and the improved matching between $\mathrm{P}$ and $\mathrm{N}$ nodes in (b).

A basic model of the switched capacitor circuit can thus be constructed as shown in Fig. 3.2, with the fixed capacitor/varactor modelled as a resistance $G_{b a n k}$ in parallel with a capacitance $C_{\text {bank }}$ both in series with the MOS switch, which is modelled as another resistor $G_{s w}$ in parallel with a capacitor $G_{s w}$. When the capacitor bank is switched, only the voltage $V_{s w}$ applied on the transistor switch is changed, so to a first degree of approximation, only the resistance associated with the transistor will change, since most of $C_{s w}$ comes from extrinsic parasitics. In reality, the changing voltage applied on the tank voltage will have some impact, as do the intrinsic parasitics of the switch, but these effects are significantly smaller than the primary effect of the change in switch resistance. 
In typical operation, the DC voltage across the switch will be very small, so the transistor typically operates either in the triode or in the cutoff region of operation. To determine the theoretical relationship between the switch conductance and the gate voltage, the square law transistor model is used. While this gives a very rough approximation due to the presence of short channel non-idealities, this gives an idea of the general trends.

$$
\begin{array}{r}
I_{D S}=\mu C_{o x} \frac{W}{L} V_{D S}\left(\left(V_{g s}-V_{T H}\right)-\frac{V_{D S}}{2}\right) \approx \mu C_{o x} \frac{W}{L} V_{D S}\left(V_{g s}-V_{T H}\right) \\
G_{s w}=\frac{I_{D S}}{V_{D S}} \approx \mu C_{o x} \frac{W}{L}\left(V_{g s}-V_{T H}\right)
\end{array}
$$

where $G_{s w}$ is the effective switch conductance, $I_{D S}$ is the drain current, W and L are the transistor's gate width and length respectively, $\mu$ is the channel mobility, $C_{o x}$ is the gate capacitance per unit area, $V_{g s}$ and $V_{d s}$ are the gate-source and gate-drain voltages respectively, and $V_{T H}$ is the transistor's threshold voltage.

When $V_{g s}-V_{T H}<0$, the transistor enters the sub-threshold region of operation, and $G_{s w}$ is effectively 0 . Thus, (3.2) shows that as $V_{s w}$ is changed from 0 to $V_{D D}, G_{s w}$ changes continuously and monotonically from 0 to $\frac{I_{D S}}{V_{D S}} \approx \mu C_{o x} \frac{W}{L}\left(V_{D D}-V_{T H}\right)$. This allows the switch to be modelled as a variable resistance whose value moves between these two values continuously.

From Fig. 3.2, the impedance of both the switch and the capacitor, as seen from the tank, can be calculated as follows:

$$
Z_{\text {total }}(s)=\left(\frac{1}{C_{s w}}+\frac{1}{C_{\text {bank }}}\right) \frac{s+\frac{G_{b a n k}+G_{s w}}{C_{b a n k}+C_{s w}}}{\left(s+\frac{G_{b a n k}}{C_{b a n k}}\right)\left(s+\frac{G_{s w}}{C_{s w}}\right)}
$$

Equation (3.3) can be simplified by noting that in general $|s| \gg\left|G_{b a n k} / C_{b a n k}\right|$ and that $C_{s w} \ll C_{\text {bank }}$, giving the following:

$$
Z_{t o t a l} \approx \frac{1}{C_{s w}} \frac{s+\frac{G_{b a n k}+G_{s w}}{C_{b a n k}}}{s\left(s+\frac{G_{s w}}{C_{s w}}\right)}=\frac{1}{s C_{s w}} \frac{s+\frac{G_{b a n k}+\mu C_{o x} \frac{W}{L}\left(V_{s w}-V_{T H}\right)}{C_{b a n k}}}{s+\frac{\mu C_{o x} \frac{W}{L}\left(V_{s w}-V_{T H}\right)}{C_{s w}}}
$$

The locations of the poles and zero in Equation (3.4) can then be analyzed. Fig. 3.3 shows a pole-zero plot of this equation. As $V_{s w}$ is changed, the pole and zero and the last term move from close to the origin when $V_{s w}$ is low to far along the negative real axis when $V_{s w}$ is large, effecting an impedance change from $1 / s C_{s w}$ to $1 / s C_{b a n k}$ with continuous intermediate values.

Unlike varactors, capacitor banks are not designed for use in its transitionary region, due 


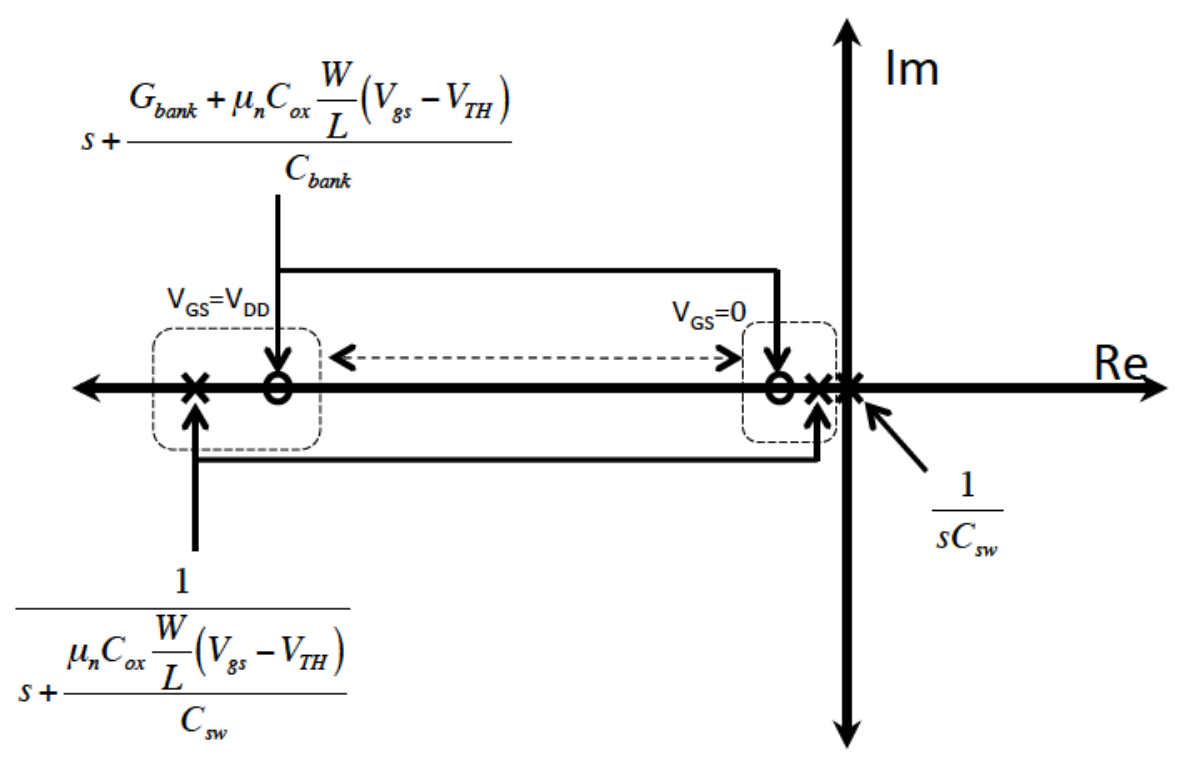

Figure 3.3: Pole-zero plot of the capacitor bank

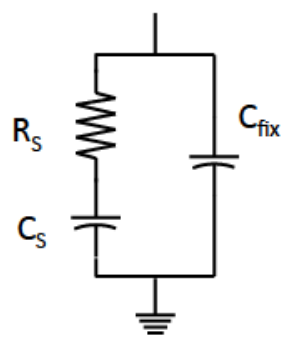

Figure 3.4: Simplified model of the capacitor bank

to the fact that in this region, the switch does not function as an ideal open circuit (with high impedance) or an ideal closed circuit (with low impedance). Since the circuit is designed to switch slowly, any region with poor performance will occur. Thus, some performance degradation may be possible and an analysis of the quality factor of the capacitor bank during switching is required.

By simplifying the model further, a more qualitative analysis of the capacitor bank can be done. This is done by using the simplified model shown in Fig 3.4, which uses a single resistor $R_{s}$ to model the switch resistance in series with a single capacitor $C_{s}$, to model the switched capacitor. Both are in parallel with a fixed capacitance $C_{f i x}$ which models the parasitics, fixed capacitance and the other capacitor banks of the circuit. As the switch is turned from on to off and vice versa, $C_{s}$ remains constant, but $R_{s}$ changes in value from $R_{o n}$ 
to $R_{\text {off }}$.

For narrow band applications, such as an oscillator, the series combination of $R_{s}$ and $C_{s}$ can be transformed into a parallel combination $R_{p}$ and $C_{p}$, whose values are frequency dependant. The relationship between the two can be determined as follows:

$$
\begin{array}{r}
Z_{s}=Z_{R s}+Z_{C s}=R_{s}+\frac{1}{j \omega C_{s}}=\frac{j \omega C_{s} R_{s}+1}{j \omega C_{s}} \\
Y_{s}=\frac{j \omega C_{s}}{1+j \omega C_{s} R_{s}}=j \frac{\omega C_{s}}{1+\omega^{2} C_{s}^{2} R_{s}^{2}}+\frac{\omega^{2} C_{s}^{2} R_{s}}{1+\omega^{2} C_{s}^{2} R_{s}^{2}} \\
Y_{T}=Y_{s}+Y_{C p}=j \omega\left(\frac{C_{s}}{1+\omega^{2} C_{s}^{2} R_{s}^{2}}+C_{p}\right)+\frac{\omega^{2} C_{s}^{2} R_{s}}{1+\omega^{2} C_{s}^{2} R_{s}^{2}}
\end{array}
$$

The real part of equation (3.7) gives the expression for the parasitic parallel conductance of the capacitor bank:

$$
G_{p}=\frac{\omega^{2} C_{s}^{2} R_{s}}{1+\omega^{2} C_{s}^{2} R_{s}^{2}}
$$

By taking the first derivative of (3.8) with respect to $R_{s}$, another expression is formed:

$$
\frac{\partial G_{p}}{\partial R_{s}}=\frac{\omega^{4} C_{s}^{4} R_{s}^{2}-\omega^{2} C_{s}^{2}}{1+2 \omega^{2} C_{s}^{2} R_{s}^{2}+\omega^{4} C_{s}^{4} R_{s}^{4}}
$$

The maximum parallel conductance can then be found when the numerator is 0 :

$$
R_{s, G \text { max }}=\frac{1}{\omega C_{s}}
$$

Then, the maximum parallel conductance can be found:

$$
G_{p, \max }=\frac{\omega C_{s}}{2}
$$

Equation (3.11) shows that the maximum parasitic conductance of the switched capacitor bank depends only on the reactance of the capacitor added and is independent of $R_{o n}$, $R_{\text {off }}$, showing that switch size does not impact this minimum value (note that the parasitic capacitance of the switch is ignored in this simplified model). Since the switch will slowly transition between on and off states, the oscillator will always spend significant time in this low Q state. Changing the switch size only changes when this state is encountered.

To minimize the effect of this low Q state, it should be noted that the parasitic conductance of the entire tank is the sum of all of the parasitic conductances added by all parts of the tank circuitry (such as inductor losses, gate resistance and output impedance of the gain circuitry, resistive losses in interconnect). This can be done by minimizing the value 
of $G_{p, \min }$ by keeping $C_{s}$ small. Thus, the amount of degradation can be controlled with capacitor bank scaling, provided that sufficient phase noise margin is available to tolerate the decreased quality factor.

Further analysis can be done by looking at the the imaginary part of equation (3.7). By taking its derivative, the following expression can be obtained:

$$
\begin{array}{r}
B_{T}=\frac{\omega C_{s}}{1+\omega^{2} C_{s}^{2} R_{s}^{2}}+\omega C_{p} \\
\frac{\partial B_{T}}{\partial R_{s}}=\frac{-2 \omega^{3} C_{s}^{3} R_{s}}{1+2 \omega^{2} C_{s}^{2} R_{s}^{2}+\omega^{4} C_{s}^{4} R_{s}^{4}}
\end{array}
$$

For all values of $R_{s}>0$, this is negative and non-zero, indicating that as $R_{s}$ is transitioned from $R_{\text {off }}$ to $R_{o n}$, a monotonic, gradual change in capacitance is seen, resulting in a gradual, monotonic shift in the resonant frequency of the LC tank.

Thus, a continuous change in capacitance can be achieved through the slow, gradual switching of conventional capacitor banks. Although this approach results in a temporary drop in tank Q factor, the use of closely spaced frequency bands (as is already the case due to the desire to minimize $\mathrm{K}_{\mathrm{VCO}}$ ) serves to limit the impact this has.

\subsubsection{PLL System Level Analysis}

The concept proposed here is the use of a slowly switched capacitor bank in order to prevent PLL cycle slipping during band switches. In order to properly design and analyze the circuits necessary to achieve this, it is critical to be able to properly model the PLL system to determine what conditions cause cycle slipping and what are the critical performance targets that the band switching circuit needs to achieve in order to prevent this.

The behaviour of the PLL with the proposed band switching circuit can be analyzed using conventional system analysis techniques by modelling the PLL in the Laplace domain. The system diagram used for this can be seen in Fig. 3.5. In this diagram, all of the standard elements of a PLL are modelled conventionally: the phase detector is treated as a sum block

with a gain block, the loop filter is modelled using a continuous-time transfer function, the VCO is modelled as a integrator with gain, and the divider is modelled as an attenuating block.

The remaining element for which a conventional model does not exist is the capacitor bank circuitry. In order to properly model the capacitor bank, an understanding of its transient behaviour is needed. Typical capacitor bank circuits are shown in in Fig. 3.6. As is evident, this circuit consists of a capacitor (which may consist of a combination of fixed capacitors 


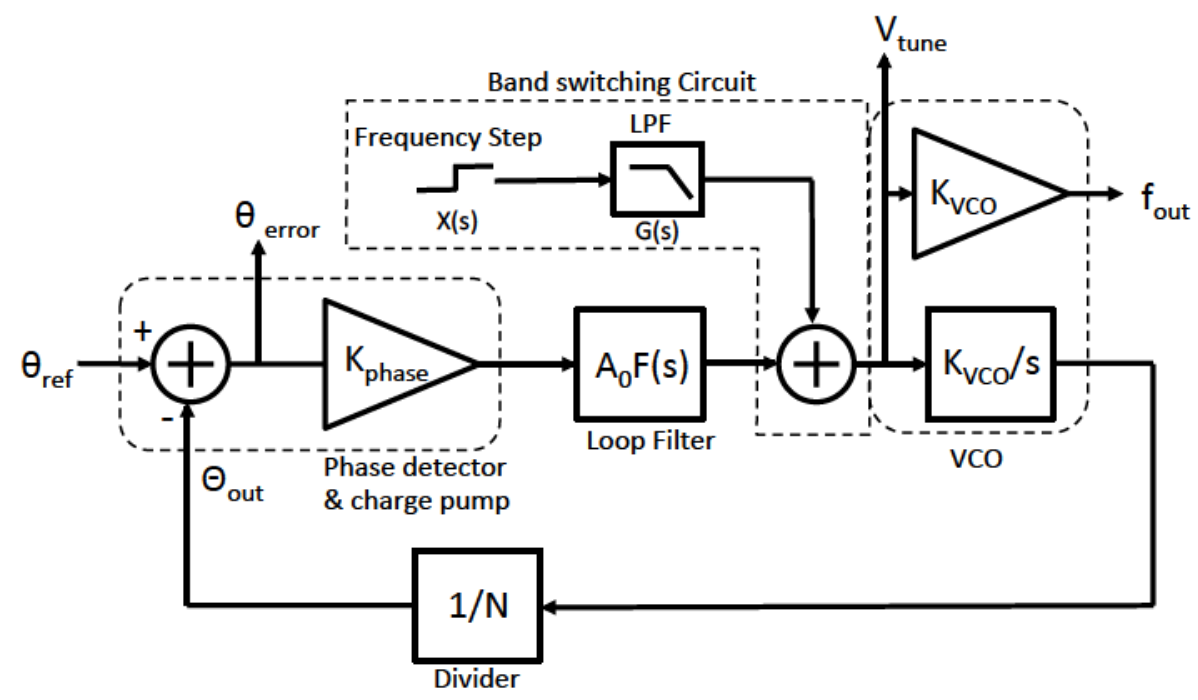

Figure 3.5: Laplace domain system diagram of the PLL used to analyze its behaviour with slow band switches

such as MIM or MOM capacitors, and/or varactors) in series with a MOS transistor which acts as a switch. When the FET switches states, the total impedance of the circuit changes from its off state impedance to its on state impedance, as described in Section 3.1.1. This causes a change in the oscillator tank impedance which results in a change in oscillation frequency.

When the capacitor bank is switched, several events take place, each with their own time constant. After the transistor is switched, there is a delay before the impedance change. This length of this delay depends on the time needed to form or deplete the MOSFET's inversion layer, which is on the same order of magnitude of the inverse of the transistor's

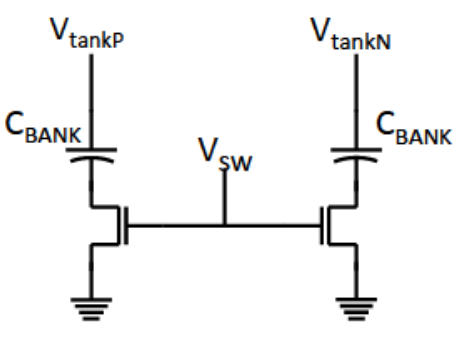

(a)

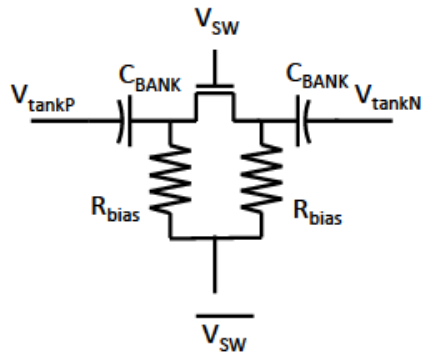

(b)

Figure 3.6: Schematic showing typical VCO capacitor bank circuits 
transit frequency $f_{T}$, which is typically on the order of tens of $\mathrm{GHz}$ or more. After this, the equivalent RC circuit (as formed by the capacitor in the bank and the transistor's switch resistance) requires time to charge and discharge. Its time constant, $1 / R C$ (where $\mathrm{R}$ is the switch resistance and $\mathrm{C}$ is the bank capacitance), is quite small (less than $10 \mathrm{ps}$ ) since the added capacitance in a single bank is small and the switch is often sized to add minimal resistance. The final important event is the response of the LC tank circuit to the added (or removed) capacitance, whereby its resonant frequency is shifted. This takes place with a time constant of $1 / \sqrt{L C}$, which is the oscillation frequency, and thus takes several periods of the oscillation frequency to settle.

Conversely, the PLL operates on a time scale much slower than any of these effects. Typically, in order to model it as a continuous time system, the loop time constant must be made larger than the clock period of the PLL reference clock, whose frequency is usually in the range of 10 to $100 \mathrm{MHz}$. As a result, from the perspective of the PLL, all of these effects occur instantaneously and the change in frequency can be modelled as being directly controlled by the change on the capacitor bank control signal. Thus, the capacitor bank modelled by a block which adds an offset to the VCO control signal. In conventional operation of the capacitor bank, the transistor is switched rapidly, and thus, the change in frequency resembles a step function. With the proposed capacitor bank slow switching, this change takes place over a long period of time. In order to capture the behaviour of the PLL under both conventional operation and the proposed capacitor bank slow switching circuit, a first order filter block $G(s)$ is applied to a step function block $X(s)$, which is then added to the VCO control signal. By varying the corner frequency of G(s), both rapid and capacitor bank slow switching can be modelled.

The size of $X(s)$ is determined by the size of the frequency shift applied by a capacitor bank, and is scaled by $K_{V C O}$, since the control signal at the point the offset is added is in volts. It thus takes the form of

$$
X(s)=\frac{\Delta f}{K_{V C O}} \frac{1}{s}
$$

where $\Delta f$ is the frequency spacing between frequency bands and $K_{V C O}$ is the $\mathrm{VCO}$ gain. Since the PLL is a closed loop system with negative feedback, after this offset is applied to the VCO control signal, the loop will attempt to compensate for the offset, returning the VCO control signal to its original value.

The value of $G(s)$ is determined by using a simple first order low-pass filter. In reality, the correct block equation will depend on the nature of the implementation of the slow switching circuit, but to a first order of approximation, the use of a first order filter will be useful in 
determining overall loop behaviour. The filter transfer function can be described as follows:

$$
G(s)=\frac{\omega_{L}}{s+\omega_{L}}
$$

where $\omega_{L}$ is the corner frequency of the low-pass filter.

When there is no band switching taking place, $X(s)$ is constant, and since $G(s)$ is placed outside of the loop, many of the loop properties, such as loop bandwidth, damping constant, noise properties and stability do not change from that of a conventional PLL. To determine the loop response when a band switch takes place, the output phase error, taken at the output of the phase detector, is examined. By using standard linear systems theory, the output phase error transfer function with respect to $X(s)$ can be determined:

$$
\frac{\theta_{\text {error }}(s)}{X(s)}=\frac{\frac{K_{V C O}}{N} s G(s)}{s^{2}+2 \zeta \omega_{n} s+\omega_{n}^{2}}
$$

where $\omega_{n}$ is the loop's natural frequency, $\zeta$ is the damping constant, and $\mathrm{N}$ is the PLL divider ratio. In the case with fast band switching, $G(s)$ does not apply any filtering, so $\omega_{L}=\infty$ making $G(s)=1$. For a step function given by Eq. (3.14), this gives a phase error of

$$
\theta_{\text {error }}(s)=\frac{\frac{\Delta f}{N}}{s^{2}+2 \zeta \omega_{n} s+\omega_{n}^{2}}
$$

This can be converted into the time domain using inverse Laplace transforms, as shown in [56]. With most PLLs, settling time is minimized, so the damping factors are set to be less than 1 . Thus, this yields the following:

$$
\theta_{\text {error }}(t)=\frac{\Delta f}{N} \frac{\sin \left(\omega_{n} \sqrt{1-\zeta^{2}} t\right)}{\omega_{n} \sqrt{1-\zeta^{2}}} e^{-\zeta \omega_{n} t}
$$

To model the effects of slow band switching, $G(s)$ is set to be a low-pass filter, as given in Eq. (3.15). The error transfer function thus becomes

$$
\theta_{\text {error }, \text { slowsw }}(s)=\frac{\omega_{L}}{s+\omega_{L}} \frac{\frac{\Delta f}{N}}{s^{2}+2 \zeta \omega_{n} s+\omega_{n}^{2}}
$$

and the transient response becomes 


$$
\begin{aligned}
& \theta_{\text {error }, \text { slowsw }}(t)=\frac{\omega_{L} \frac{\Delta f}{N}}{-\omega_{L}^{2}+2 \zeta \omega_{L} \omega_{n} s+\omega_{n}^{2}}\left[-e^{-\omega_{L} t}+\cos \left(\omega_{n} \sqrt{1-\zeta^{2}} t\right) e^{-\zeta \omega_{n} t}+\right. \\
& \left.+\left(\zeta \omega_{n}-\omega_{L}\right) \frac{\sin \left(\omega_{n} \sqrt{1-\zeta^{2}} t\right)}{\omega_{n} \sqrt{1-\zeta^{2}}} e^{-\zeta \omega_{n} t}\right]
\end{aligned}
$$

Similarly, the instantaneous frequency error can also be calculated. This is done by looking at the input control signal to the VCO and scaling it by $K_{V C O}$. This relies on the assumption that the VCO reacts instantly to changes in the control signal, but as was discussed in Section 3.1.2, this assumption is a reasonable one since the PLL operates on a much larger time scale than any of the time constants within the VCO. The general form of the frequency error can be found as follows:

$$
\Delta f_{\text {out }}(s)=K_{V C O} \frac{s^{2} X(s) G(s)}{s^{2}+2 \zeta \omega_{n} s+\omega_{n}^{2}}
$$

With appropriate values for $X(s)$ and $G(s)$, the transient responses can be calculated. The case with conventional band switching can be seen in (3.22), while the case with slow switching can be seen in (3.23).

$$
\begin{gathered}
\Delta f_{\text {out }}(t)=\Delta f\left[\cos \left(\omega_{n} \sqrt{1-\zeta^{2}} t\right) e^{-\zeta \omega_{n} t}-\frac{\zeta}{\sqrt{1-\zeta^{2}}} \sin \left(\omega_{n} \sqrt{1-\zeta^{2}} t\right) e^{-\zeta \omega_{n} t}\right] \\
\Delta f_{\text {out }, \text { slows } w}(t)=\frac{\Delta f \omega_{L}}{2 \zeta \omega_{n}-\omega_{L}-\frac{\omega_{n}^{2}}{\omega_{L}}}\left[\begin{array}{c}
e^{-\omega_{L} t}-\cos \left(\omega_{n} \sqrt{1-\zeta^{2}} t\right) e^{-\zeta \omega_{n} t}+ \\
\left.+\frac{\zeta \omega_{L}-\omega_{n}}{\omega_{L} \sqrt{1-\zeta^{2}}} \sin \left(\omega_{n} \sqrt{1-\zeta^{2}} t\right) e^{-\zeta \omega_{n} t}\right]
\end{array}\right.
\end{gathered}
$$

A plot showing the normalized transient phase error and normalized transient frequency error of a simulated PLL (with varying values of $\omega_{L}$ ) can be seen in Fig. 3.7.

The transient responses in (3.18) and (3.20) can be compared to determine the theoretical impact that the slow switching circuit has on the transient response of the filter. The 3 terms in the brackets in Eq. (3.20) provide different contributions to the overall response of the system, depending on the value of $\omega_{L}$. Since the last term scales by an additional factor of $\omega_{L}$, it dominates the expression when $\omega_{L} \gg \omega_{n}$, and when simplified, gives the same 


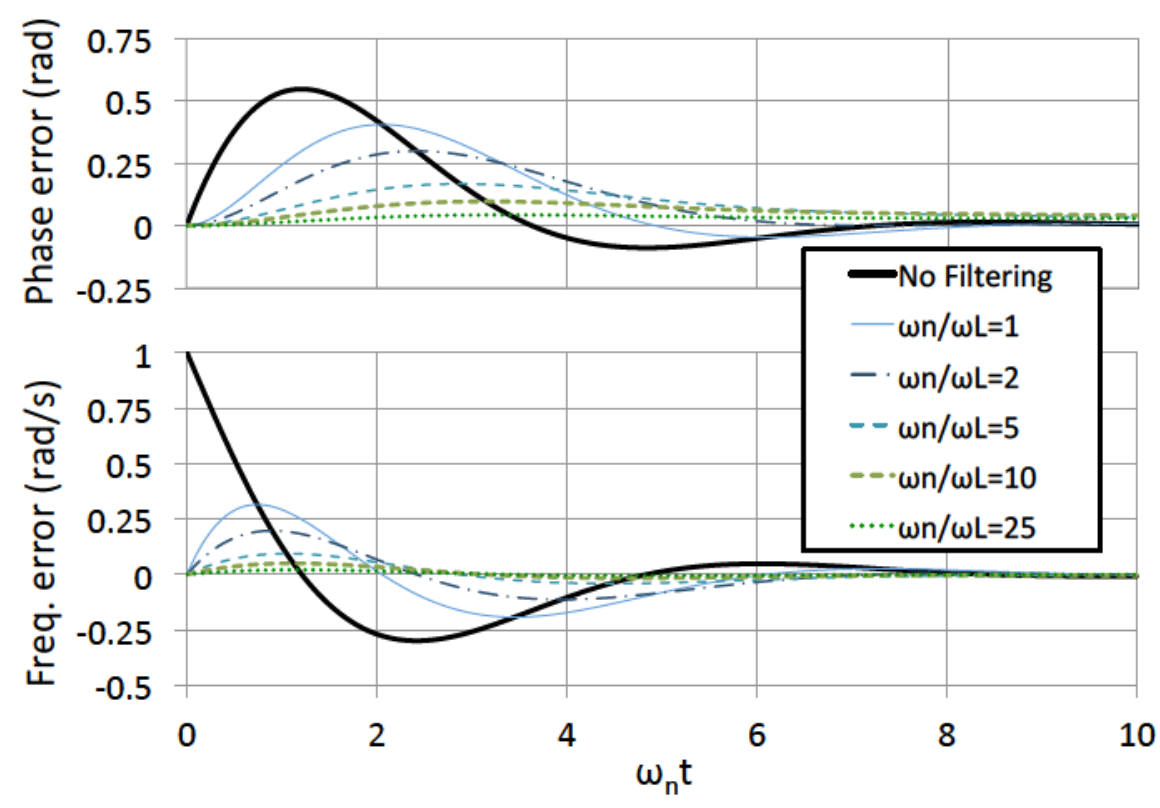

Figure 3.7: Plot of the simulated scaled transient phase and frequency errors with conventional band switching and slow switching with varying filter bandwidths

expression as in (3.18), as would be expected with large values of $\omega_{L}$, since that indicates a large filter bandwidth which effectively filters out nothing. Similarly, as $\omega_{L}$ approaches $\omega_{n}$, the terms all play a significant role and when $\omega_{L} \ll \omega_{n}$, the first two terms dominate the expression. In typical cases of the proposed filtering circuit, $\omega_{L}$ will be much smaller than $\omega_{n}$, so this last case is given more consideration. With these simplifications, it becomes

$$
\theta_{\text {error }, \text { slowsw }}(t) \approx \frac{\omega_{L} \frac{\Delta f}{N}}{\omega_{n}^{2}}\left[-e^{-\omega_{L} t}+\cos \left(\omega_{n} \sqrt{1-\zeta^{2}} t\right) e^{-\zeta \omega_{n} t}\right]
$$

Since $\omega_{L} \ll \omega_{n}$, the cosine term rapidly decreases, leaving only the first exponential term, with a time constant of $1 / \omega_{L}$, as would be expected. Thus, the system takes longer to recover from the phase error. However, it can be seen that there is an additional factor of $\omega_{L} / \omega_{n}$, which reduces the maximum phase offset. A similar analysis with the frequency error, comparing expressions (3.22) and (3.23), giving the same set of relations.

The key insight gained by these theoretical equations is the fact that decreasing $\omega_{L} / \omega_{n}$ allows the maximum phase error and maximum frequency error to be decreased, with the limiting value only dependant on how low $\omega_{L}$ can be made. This is shown in Fig. 3.8, which shows a plot of the theoretical attenuation of the maximum phase and frequency error relative to the phase and frequency error in the conventional switching case over different values of 


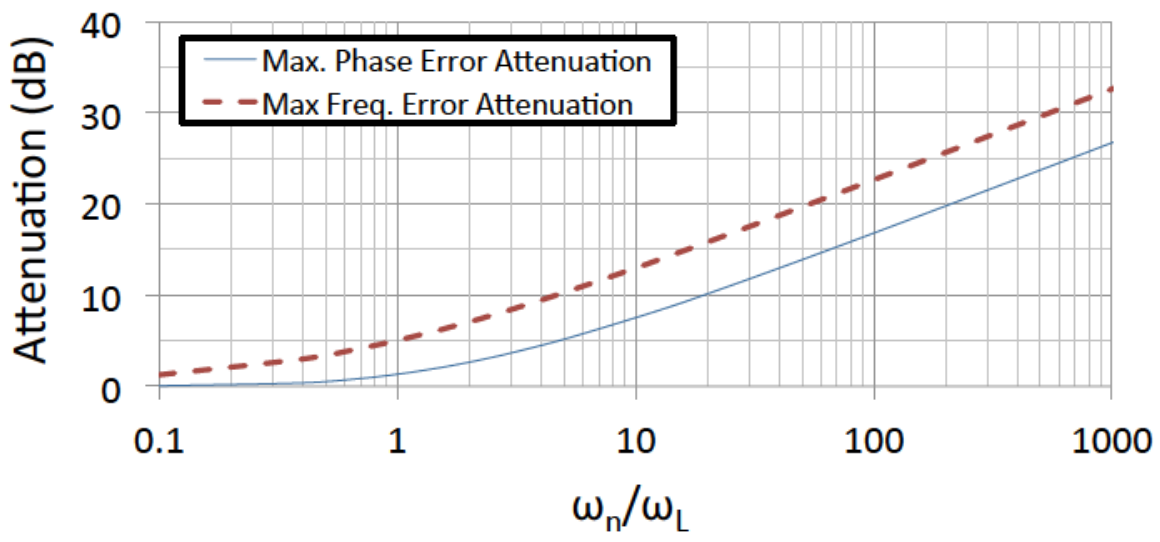

Figure 3.8: Plot of the attenuation of maximum phase and frequency errors with different filter bandwidths

$\omega_{L} / \omega_{n}$. This plot again shows that the phase error and frequency error attenuation increases with decreasing $\omega_{L} / \omega_{n}$. As $\omega_{L} / \omega_{n}$ drops below 0.1 , the maximum phase and frequency errors are reduced proportionally to $\omega_{L} / \omega_{n}$. Thus, a specification of a maximum phase error in order to avoid cycle slipping can be used to determine what maximum frequency band switching speed can be used, and appropriate circuits used to implement this as necessary.

\subsubsection{Capacitor Bank switching circuit}

Previously, it has been shown that a typical VCO capacitor bank can be made to exhibit gradual changes in frequency, provided the control voltage is switched slowly enough, and that the PLL is able to compensate for large changes in frequency due to band switching, provided the change is introduced slowly enough. Thus, these two concepts can be combined together to enable a PLL that is able to switch frequency bands without cycle slipping, thus ensuring that no phase offsets are introduced during frequency band switches. This section discusses the proposed circuitry to implement this.

As shown previously in 3.1.1, slowly changing the voltage on a capacitor bank causes a gradual change in the capacitor bank capacitance. Should this be done gradually enough, the frequency shift that would be caused by the additional capacitance will occur slowly enough for the loop to compensate, allowing for near constant frequency, and minimizing phase transients, preventing cycle slipping, as shown in Section 3.1.2. A critical issue lies in the generation of the required slowly switched capacitor bank control signal, however, which is made difficult by several issues. The primary issue is the time scale required for the switching circuit. In order for the loop to be able to compensate for the added capacitance added by the capacitor bank, the voltage applied to the switch must change very slowly- a 
total rise/fall time that must be larger than the loops time constant (as indicated by the previously given constraint that $\left.\omega_{L} \ll \omega_{n}\right)$. In many PLLs, the loop bandwidth is in the range a $100-500 \mathrm{kHz}$, giving a loop time constant in the range of 2 to $10 \mu \mathrm{s}$. Thus, the rise/fall time on capacitor bank voltage must be $50 \mu$ s or more to prevent cycle slipping. This is compounded by the fact that typical VCOs will have many banks of capacitors, and that the slow switching transient waveform would be required for all of the banks.

Creating a waveform with a large time constant is difficult to apply using conventional methods. Using an $\mathrm{RC}$ filter would require tremendously large $\mathrm{R}$ and $\mathrm{C}$ values. PLL loop filters are often implemented off-chip for this reason, and even implementations using on-chip loop filters require massive area to implement this filter. To create a larger time constant, substantially larger passive components would be required, making on-chip implementation highly impractical. Off-chip implementation is also difficult due to the number of filters that would be required (one per capacitor bank). Ignoring the difficult and the increased cost of placing all of the components in the package or on the PCB, the number of pads or bumps that would be required would also be impractically large.

A proposed solution is the use of a DAC to generate the waveforms, as can be seen in [153]. This has many practical benefits since implementing large time constants in the digital domain is far easier and requires less area. However, practical implementations of a DAC based capacitor bank switching circuit are still not trivial. In order to properly create the switching waveform, a high DAC resolution and/or a reconstruction filter with a very low corner frequency is required to prevent the DAC output steps from creating additional noise which could be up-converted as phase noise or even cause loss of lock if the steps are too large. There is also difficulty in creating separate waveforms for each capacitor bank, requiring multiple DACs, or the use of a switching matrix, as seen in [153]. Finally, there is also the increased power consumption and area required by the DAC, and the fact that there is considerable design overhead required to implement the DAC.

The following section describes a proposed solution to this problem by using sub-threshold current limited transistors to implement long charge and discharge times of an RC circuit. This implements a circuit with long rise and fall times, with minimal requirements for area and power.

\subsubsection{Sub-threshold limited inverter}

A possible solution to the issues discussed previously is the use of sub-threshold currents. When operated in the sub-threshold region of operation, MOSFETs exhibit very low drain currents at moderate voltages, effectively implementing very high impedances. As described 
in [185], the current in a MOSFET operating in the sub-threshold region of operation is as follows:

$$
I_{D}=I_{0} e^{\frac{V_{G}}{n V_{T}}}\left(e^{-\frac{V_{S}}{V_{T}}}-e^{-\frac{V_{D}}{V_{T}}}\right)
$$

where $I_{0}$ is the saturation current, $V_{T}$ is the thermal voltage, $\mathrm{n}$ is the ideality factor, and $V_{G}, V_{S}$, and $V_{D}$ are the gate, source and drain potentials respectively. Since the drain current has an exponential relationship to gate-source voltage, very small currents with moderate voltages.

One possible way of taking advantage of this is to use current mirrors biased in the subthreshold region. The mirrored currents can be used to bias conventional analog circuitry and effectively implement high impedances. The exponential relationship between current and voltage in the sub-threshold region makes it feasible to practically implement the current mirror bias line.

This concept is used for the capacitor bank slow switching circuit. The schematic of the circuit used for this is shown in Fig. 3.9. In conventional designs, the digital control signal for the capacitor bank is fed to the switch transistors MV1 and MV2. To reduce the switching speed, this signal is instead fed through the 2 inverters formed by transistors M3-M6, which are current-limited by the transistors M1-M2 and M7-M8. These transistors are biased at sub-threshold current levels with voltages Vbias-P and Vbias-N respectively, which are generated using the chain of diode-connected transistors MP1, MP2, ..., MN2, MN1 connected between VDD (the supply voltage) to ground. Provided the number of transistors in this chain multiplied by the threshold voltage is greater than the supply voltage, the transistors will all operate in the sub-threshold region, and the current will be very small. The gate voltages from MP1 and MN1 can then be used to generate the bias voltages Vbias-P and Vbias-N respectively in order to bias current mirrors at sub-threshold levels. The current-limited inverters thus charge and discharge their load capacitances using sub-threshold currents, greatly increasing the rise and fall times without extra passive components.

The inverters can also be restored to normal operation to achieve fast capacitor bank switching if Vbias-P and Vbias-N are shorted to ground and VDD respectively, which is done by switch transistors MS1-MS4, which are controlled by Vetrl.

When the capacitor bank control signal is fully switched, the effect of the high impedances of transistors M2 and M8 are minimal, as the voltage gain of the switch transistors is very low, and the impedance of the capacitor bank is largely constant over gate voltage when fully switched, as will be shown in Fig. 4.18. As a result, this prevents the noise generated 


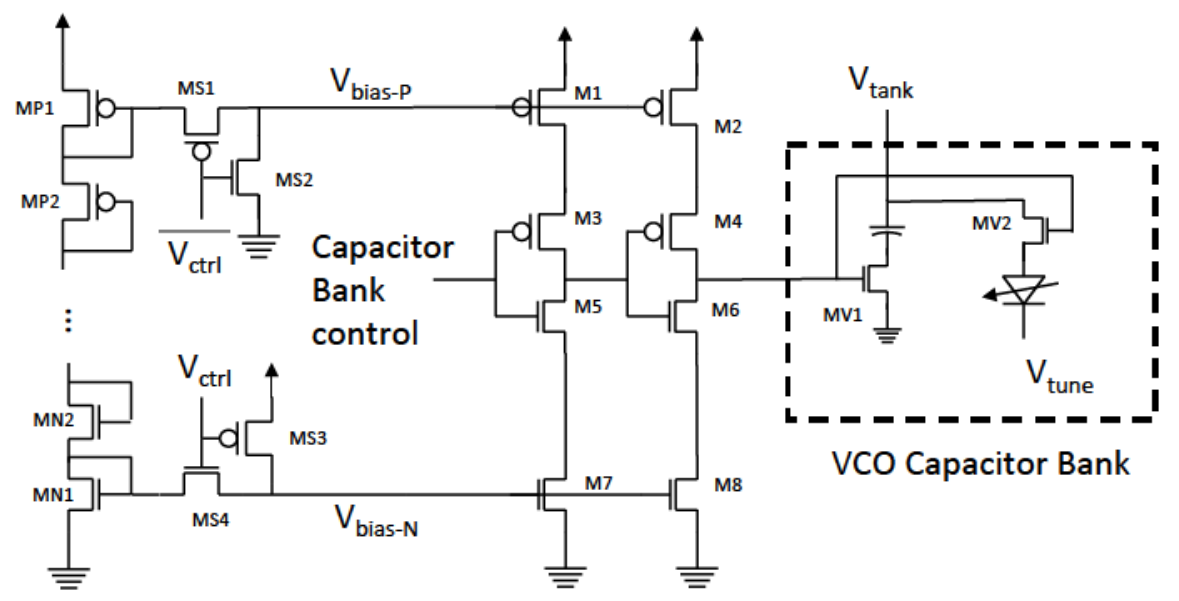

Figure 3.9: Schematic of the capacitor bank slow switching circuit

by the sub-threshold transistors from being converted into phase noise when the circuit is fully switched.

The entire circuit requires 2 bias lines, while a separate set of current-limited inverters is needed for each separate capacitor bank. As only 8 small transistors are required per each bank and no passive components are needed, the area required is minimal.

Using the expression for the sub-threshold current (3.25), the theoretical behaviour of this circuit can be determined. With a chain of $\mathrm{N}$ diode connected transistors from ground to VDD and with some simplifying assumptions, the expression can be rewritten as

$$
I_{D}=I_{0} e^{\frac{V_{D} D}{n N V_{T}}}
$$

To determine how well this expression models the behaviour of the transistors, the diode line is simulated in the IBM $0.18 \mu \mathrm{m}$ BiCMOS process that will be used to implement the design. One potential issue with sub-threshold circuits is the presence of process variation. Since MOSFETs are not commonly used in the sub-threshold region of operation, the process controls are likely not optimized for consistent behaviour from corner to corner, and thus significant variations may be encountered. Thus, the extreme functional fast-fast and functional slow-slow corners are examined as well. Fig. 3.10 shows a comparison between the theoretical transistor current and the simulated current for these corners with a varying number of diode connected transistors. For the theoretical plot, the parameters $I_{0}$ and $n$ are not directly available, and thus are chosen empirically.

The current through the diode line can then be used to estimate the rise and fall time of the inverter circuit. When the sub-threshold switching circuit is active, the inverter transistors largely act as switches, directing the sub-threshold into or out of its load. Due to 


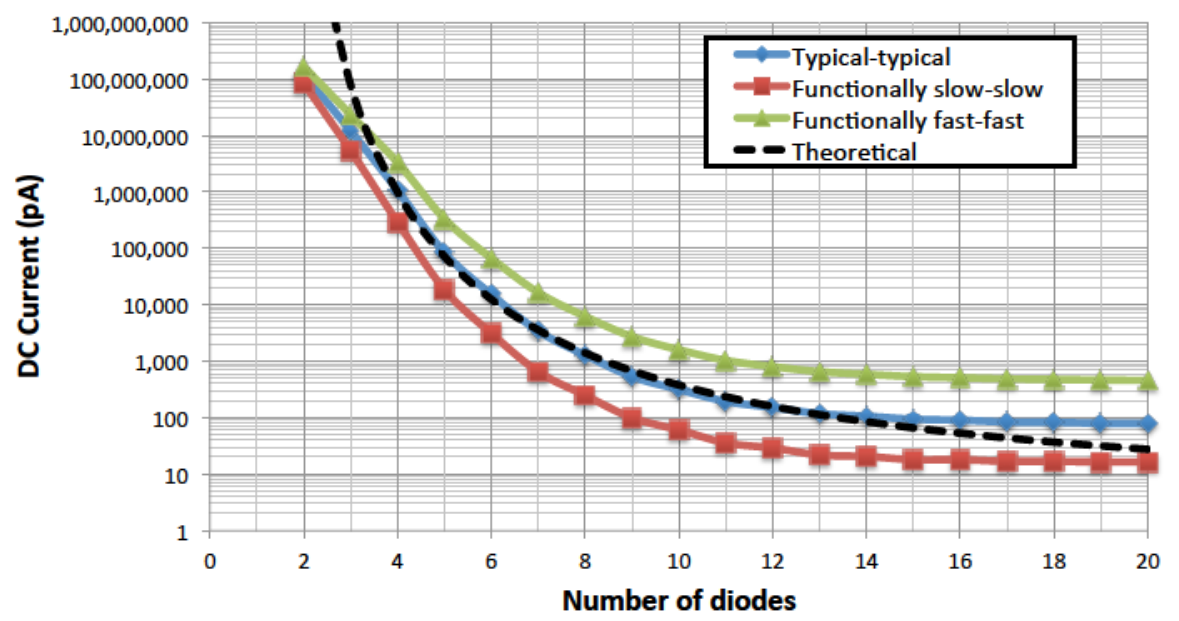

Figure 3.10: Comparison of the theoretical and simulated current in a chain of diode connected MOSFETs

the small gate voltages used, the sub-threshold transistors act almost completely as a current source, and thus drive a constant current out of or into the load, and thus the circuit can be treated as being slew rate limited. Using an estimate of the load consisting of the transistor switch and its wiring capacitance, the slew rate and the rise/fall time can be calculated.

With slew rate limited capacitive loads, the load is charged or discharged by constant current, resulting in a linear change in voltage over a given time interval proportional to the current.

$$
\Delta V=C I \Delta t
$$

With a real current sources, the transistor will not be able to deliver a constant current throughout the entire charge-discharge cycle. Towards the end of the cycle, the drain-source voltage decreases and eventually the transistor is driven into the triode region of operation, slowing down the charging/discharging cycle and changing the response to be more like an $\mathrm{RC}$ circuit. However, this effect occurs for only a small portion of the charge/discharge time, and is assumed to be negligible. If the circuit is assumed to be completely slew rate limited, and if the load capacitance is driven from a value of 0 to $V_{D D}$ (or vice versa), the charge/discharge time can be found by combining expressions (3.26) and (3.27):

$$
t_{\text {rise } / \text { fall }}=\frac{C_{L o a d} V_{D D}}{I_{0}} e^{-\frac{V_{D D}}{n N V_{T}}}
$$

As is evident, the rise and fall times increase exponentially with increasing number of diode connected transistors. A plot of the simulated $10 \%$ to $90 \%$ rise and fall times of the 


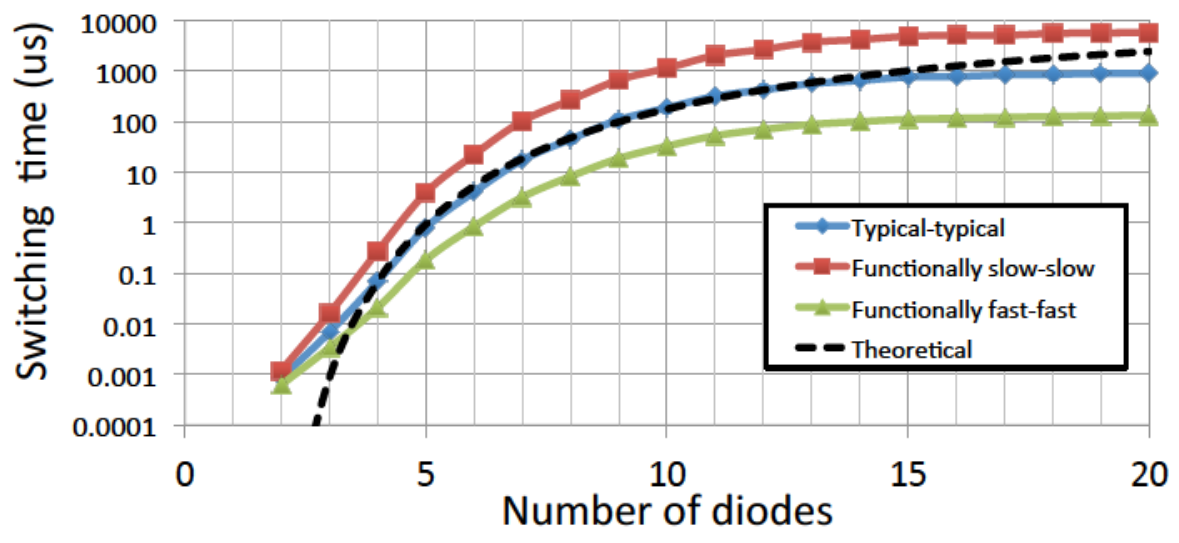

Figure 3.11: Comparison of the theoretical and simulated rise/fall time of the sub-threshold current limited inverter with varying number of diode connected transistor in the bias line

circuit with a $20 \mathrm{fF}$ load over the nominal, functional fast-fast and functional slow-slow corners compared with the theoretical rise/fall times can be seen in Fig 3.11. The $20 \mathrm{fF}$ load represents the approximate wiring parasitic capacitance and the load capacitance of the switch.

It is apparent from Fig 3.11 that process variation plays a significant role on the performance of the circuit. From the typical to either of the process extremes, the rise/fall time varies by about a factor of 10 in the subthreshold region. In the initial test chip, this is taken into account by designing the circuit such that in the worst case corner cycle slipping is prevented while avoiding as much as possible the parasitic dominated region. It should also be noted that results shown in Fig 3.11 are for a typical supply voltage and temperature. As will be shown in Section 6.1, the rise/fall time also heavily depends on these factors as well. Thus to fully compensate for this, a calibration circuit will be required, an example of which will be shown in Section 6.1. For the initial test chip, the sensitivity to temperature and voltage was a risk that was considered to be minimal for the purposes of a proof of concept test chip, since both these variables could be controlled during measurement.

Since the primary vulnerability of this circuit is to extreme transistor behaviour and not device mismatch, the use of process corners was considered sufficient and Monte Carlo simulations were not conducted.

As can be seen in both Fig. 3.10 and Fig. 3.11, the theoretical model accurately predicts the simulation results for a chain of 3 to 14 transistors. For a chain using fewer than 3 transistors, the transistors are no longer operating in the sub-threshold region of operation, and thus the exponential model cannot be used to predict the drain current. For a chain of more than 14 diode connected transistors, the current drops to very low levels, and thus 
other parasitic p-n junctions (such as the source and drain diffusions into the bulk) will start to play a significant role. Thus, operating with more than 14 diode connected transistors may introduce significant risks of unpredictable behaviour leading to poor performance. In addition, because MOSFETs are not usually used in the sub-threshold region of operation, it is probable that the transistors' behaviour may not be well characterized in the models. Thus, the design of the diode chain must take these into account by using a robust topology that does not have a strong dependance on precise transistor behaviour. Thus, the design philosophy used was to use as many diode-connected transistors as possible while avoiding as much as possible the parasitic dominated region.

Fig. 3.11 shows that using 14 diode connected transistors allows for a rise/fall time to range from $100 \mu$ s to more than $1 \mathrm{~ms}$. For typical low-noise PLLs, the loop bandwidth is typically in the $100-500 \mathrm{kHz}$ range. Thus, the switching circuit is able to achieve switching times that are slow relative to the PLL's time constant, and should thus be able to achieve band switching with minimal transient effects. While using a large number of diode connected transistors allows for large settling times, resulting in small phase and frequency errors in the PLL, it may be possible that this is still insufficient to prevent cycle slipping. Alternatively, it may be desirable to reduce phase or frequency errors to an even lower degree to what the sub-threshold current limited inverter is able to do. As shown in expression (3.28), the rise and fall time can also be increased by increasing the load capacitance. The example shown in Fig. 3.11 uses a modest $20 \mathrm{fF}$, which was approximately the total parasitic wiring and load capacitance in the circuit. Further increases in this number, by adding an discrete capacitor could easily increase by 10 times or more.

To determine what effect the slow switching circuit has on the PLL, and to determine how much filtering and/or additional load capacitance is required to meet a given system specification, analysis of the PLL is required. While the theoretical equations can be used to theoretically determine the maximum tolerable phase shift, system level simulations are generally required to gain a better estimate in order to account for non-ideal PLL behaviour that result from it being a sampled system and from non-linear effects that result from that. In a simulation of the PLL run in MATLAB with estimates for the filter coefficients and circuit models, a maximum phase shift of $2 \pi$ can be seen at the phase detector before the PLL enters cycle slipping. The PLL has a frequency band spacing of approximately $20 \mathrm{MHz}$, and simulations indicate that this is sufficient to cause cycle slipping after band switching, but that cycle slipping can be avoided completely with a phase error reduction of $65 \%$. From Fig 3.8, this shows that $\omega_{L} / \omega_{n}$ has a maximum value of 4 . With a PLL system bandwidth of $200 \mathrm{kHz}$, this means that filter bandwidth requires a cutoff below $50 \mathrm{kHz}$.

Translating this to circuit parameters requires a degree of approximation since the sub- 
threshold current limited inverters and the capacitor bank circuit do not exactly implement an ideal first order filter on the frequency shift. This is partially due to the slew rate limited behaviour of the inverter, but also due to the non-linear transfer function of the capacitor bank when comparing capacitance to switch voltage (as can be seen in Fig. 4.18). Thus additional margin is required to take this into account. Noting that change in capacitance takes place only from $0.4 \mathrm{~V}$ to $0.6 \mathrm{~V}$ in Fig. 4.18, and that a low-pass filter with corner frequency of $\omega_{L}$ would have a time constant of $3 \mu$ s (giving a rise/fall time of $6 \mu \mathrm{s}$ ), a total minimum rise/fall time of $60 \mu$ s would be required. Comparing this value to Fig. 3.11, it can be seen that in the worse case condition (a functional fast-fast corner), this can be achieved if a diode line containing 9 diode-connected transistors is used. However, if instead the maximum number of 14 is used, significant margin is achieved, although this can still be done to further reduce the phase error. For the test chip used to demonstrated this concept, the larger number of diode connected transistors is used.

\subsection{Alternative Solutions}

The discussion in this chapter has described the theoretical basis behind the research in this thesis, by which a PLL frequency synthesizer is able to switch frequency bands without cycle slipping by suppressing the switching transients through the use of slowly switched capacitor banks by way of sub-threshold transistors. Some potential alternative methods to accomplish this are described in this section.

\subsubsection{Multi-state PFD and charge pump}

Cycle slipping occurs when the phase difference between the divided down VCO and the reference clock exceed the linear input range of the phase detector. A tri-state phase/frequency detector has a larger linear range than logic gate based phase detector since it has multiple state and is able to remember if one signal is faster than than the other by counting up to one doubled edge. By adding multiple states, as shown in 3.12 , the state machine can count multiple edges, increasing the linear input range. If enough states are added, cycle slipping can be prevented when switching frequency bands. This also has the added benefit of reducing lock times.

However, the implementation of this solution poses some issues. The linear range of the PFD increases linearly with the number of states, so potentially a large number of states are needed. The charge pump must be able to accommodate for these additional states, since the current must be proportionally larger in the states representing larger phase offsets. This can 


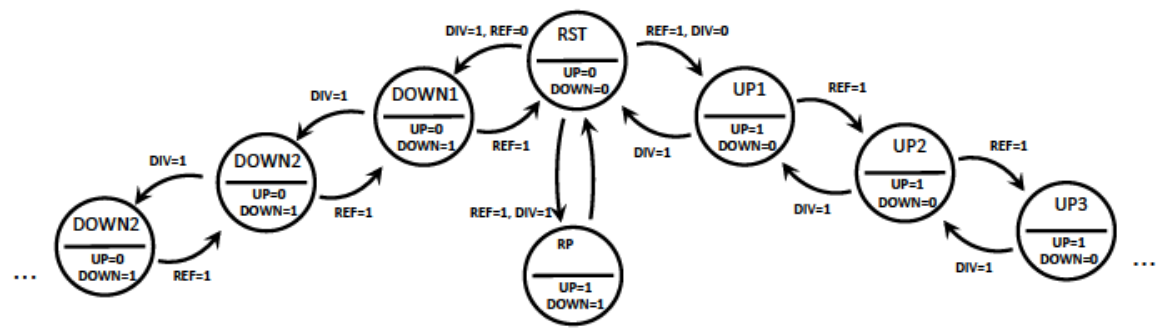

Figure 3.12: Design of a expanded multi-state phase-frequency detector

increase the non-linearities associated with the charge pump, and add more parasitics which can reduce its output current rise/fall times, which can add spurs or noise in the PLL output. Additional states in the PFD also loads the input clock signals more heavily and potentially requires more signal buffering. This can introduce more input jitter or timing offsets that can increase the output noise or add unwanted dead zones to the PFD characteristic.

So while this solution can prevent cycle slipping from occurring, it comes at the expense of some potential performance degradation during normal operation. In addition, since this method does not reduce the switching transients themselves (only increasing the size of transient allowed before cycle slipping), the PLL output will still see the full effect of the transient itself.

\subsubsection{Dynamic loop bandwidth}

Another possible solution is the dynamic variation of the PLL loop bandwidth, increasing it temporarily when performing a frequency band switch. The responsiveness of the loop to perturbations is proportional to the loop bandwidth, so increasing the loop bandwidth allows the loop to response faster to the transient effects that a band switch would introduce, and would increase the maximum allowable frequency step seen before cycle slipping occurs. If the lop bandwidth were to be sufficiently increased just prior to the band switch (and reverted back just afterwards) cycle slipping could, in theory, be prevented.

However, increasing the PLL bandwidth increases the PLL noise, both in-band phase noise (since the operating PLL bandwidth is chosen to optimize to reduce output jitter) and out-of-band spur suppression. Also this is temporary, this could cause adverse problems in the system the PLL is in. In addition, the sampled nature of the PLL limits the amount by which the bandwidth can be increased. If the bandwidth is increased beyond 0.1 times the reference clock frequency, the PLL may become unstable [56]. Thus, it may not be possible to increase the loop bandwidth by an amount large enough to prevent cycle slipping. 


\subsubsection{DAC generated switch control voltage}

This thesis proposes slowly switching a capacitor bank to achieve slow frequency band switches. The capacitor bank control signal is generated using a sub-threshold limited circuit. An alternative to this is to generate a digitally controlled signal using a DAC, as shown in $[154,155,157,158]$. This method increases the complexity of the solution, requiring more transistors for the DAC and potentially a switching matrix required to be able to apply the DAC generated voltage to the numerous capacitor banks. The DAC itself can require a large number of output bits to be able to achieve the sufficiently gradual output characteristic as well as have a reasonably low output noise. Thus, this solution is likely feasible with very small geometry process nodes, and may be difficult to implement in the larger geometry nodes more commonly used in analog design.

In addition, the solution presented here is compatible with a DAC. In a later section, it will be shown that the switching time to be increased arbitrarily by using a pulse-width modulated control signal with a slowly varying duty cycle (which can also be improved with a delta-sigma modulator to prevent spurs). The use of the sub-threshold limited transistors allows this solution to be implemented with relatively simpler and lower power digital logic operating at the speed of the reference clock. 


\section{Chapter 4}

\section{Test chip design}

The concepts discussed in the previous chapter demonstrates the theoretical basis behind a capacitor bank slow switching circuit, which allows a VCO to switch frequency bands while allowing the PLL to maintain phase lock (with a small phase error remaining). In order to demonstrate this concept, the circuit is implemented in an actual frequency synthesizer. This chapter will discuss the implementation details of this test chip.

One of the key advantages of the proposed circuit is the ease of implementation in an existing design. This is important in many industrial applications, since reuse of proven designs is common to improve design workflows. In addition, another key advantage of the proposed circuit is its minimal affect on phase noise. While this seems evident looking at the theoretical equations governing the circuit behaviour, a low jitter PLL is needed to demonstrate this. Thus, the design of the test chip is based off of an existing frequency synthesizer used for low jitter timing applications. To minimize design work and to demonstrate feasibility of integration into an existing design, only the oscillator and the first stage of output buffering is modified, with the rest of the test chip remaining identical to the commercial part it is based off of.

The following sections look at the design of the test chip, including information about the process used to implement it, the architecture of test chip itself, and the design of individual components on the chip.

It should be noted that during the design process, a much more detailed and thorough set of simulations were conducted. While many of these simulation results are included in this thesis, many others are no longer accessible, since the partner company this research was conducted with has since experienced financial difficulties and declared bankruptcy. 


\subsection{Specifications}

\subsubsection{Pre-existing synthesizer}

The design used to test the proposed concepts is based on a pre-existing frequency synthesizer chip used as a timing part in high performance SERDES applications. The use of this chip as a baseline design that is appropriately modified has several advantages, such as lower design overhead, the use of proven design blocks, and the demonstrating that the proposed concept can be easily adapted to existing circuitry. This leads to certain design constraints (such as the use of the same process, limitations in layout strategies, number of pads and digital controls). Some of the relevant specifications for the pre-existing chip that must be maintained for the test chip include the following:

- the process used was the IBM 7WL $0.180 \mu \mathrm{m}$ SiGe BiCMOS process

- the in-band loop components were designed with a $\mathrm{K}_{\mathrm{VCO}}$ of $150 \mathrm{MHz} / \mathrm{V}$ in mind

- the frequency range of the $\mathrm{VCO}$ was approximately $3 \mathrm{GHz}$ to $4 \mathrm{GHz}$

- the integrated jitter of the PLL is under $1 \mathrm{ps}$ for when operating with a center frequency in the $100 \mathrm{MHz}$ to $1 \mathrm{GHz}$ range

\subsubsection{Test chip}

The design of the test chip is intended to show the feasibility of the concepts discussed in the previous chapter, and thus certain requirements can be set for it.

- in order to properly demonstrate the concept, cycle slipping must be prevented when slow frequency band switching is enabled.

- the implementation can use a larger geometry CMOS process to lower implementation costs. This will also demonstrate a minimal dependancy on a high performance process, which is important as digital assisted analog is more difficult in these processes,

- in order to demonstrate that the concept has negligible effect on the performance of the frequency synthesizer, the frequency synthesizer should have low noise and jitter, be power efficient, and have a wide tuning range

- the frequency synthesizer should be completely self contained to simplify the measurement process. Ideally, it should be packaged or bonded on a PCB, there should be a 
digital interface to allow control of the pins, and the output should be buffered to a large signal amplitude.

- the slow switching circuit must have controllable pins to allow the slow switching to be disabled or enabled at will to allow comparisons to be made between the two.

\subsection{Process information}

The process used to manufacture the chip is the IBM 7WL 0.180 $\mu \mathrm{m}$ SiGe BiCMOS process. As is typical with BiCMOS processes, the process allows the use of NMOS and PMOS MOSFETs as well as NPN and PNP bipolar transistors (although only the NPN transistors are manufactured with the higher performance vertical structure). To illustrate some of the general performance of process, the simulated DC current-voltage characteristic curve for a 20 finger $20 \mu \mathrm{m}$ NMOS transistor is shown in Fig. 4.1 and its simulated RF performance (using RF models from the kit) is shown in Fig. 4.2. Both plots are shown in terms of current density. The drain current of the PMOS transistors is similar, but approximately 2.5 times less than a similarly biased NMOS transistor.

The RF performance of the NMOS transistor shows a relatively low $f_{T}$ compared to modern processes. The $f_{T}$ was calculated using s-parameter simulations and interpolating the results around 10 to $20 \mathrm{GHz}$ to account for poor model accuracy at higher frequencies. The high value of $f_{M A X}$ and the low $N F_{\min }$ are likely indicative of the fact that gate resistance is not fully characterized in the model used which compromises the accuracy of these results.

S-parameter simulations of CMOS transistors also tend to produce low minimum noise figure values, which are typically not attainable in real life circuits once parasitics and accurate models of passives are simulated. The performance of the NPN transistors is similar to the CMOS transistors, with an $f_{T}$ of $60 \mathrm{GHz}$ (although the more expensive $7 \mathrm{HP}$ process variant NPN transistors with an $f_{T}$ of $120 \mathrm{GHz}$ ). However, this is sufficient to provide the required $\mathrm{RF}$ performance for this application at a low cost. In this design, bipolar transistors are used for CML cells, buffers, and the output drivers, where they provide high output power with a better efficiency than could be achieved with an equivalent CMOS solution

As is typical in BiCMOS design, the benefits of both the bipolar transistors and CMOS transistors can be leveraged together, with CMOS transistors extensively used for high density digital logic, while the bipolar is used for high power buffers and drivers.

In addition to the aforementioned active devices, the process also has diode varactors and AMOS varactors for tuning, thick upper metal layers with shallow trench isolation for higher quality factor on-chip inductors, and dual-MIM capacitors for high density capacitors. 


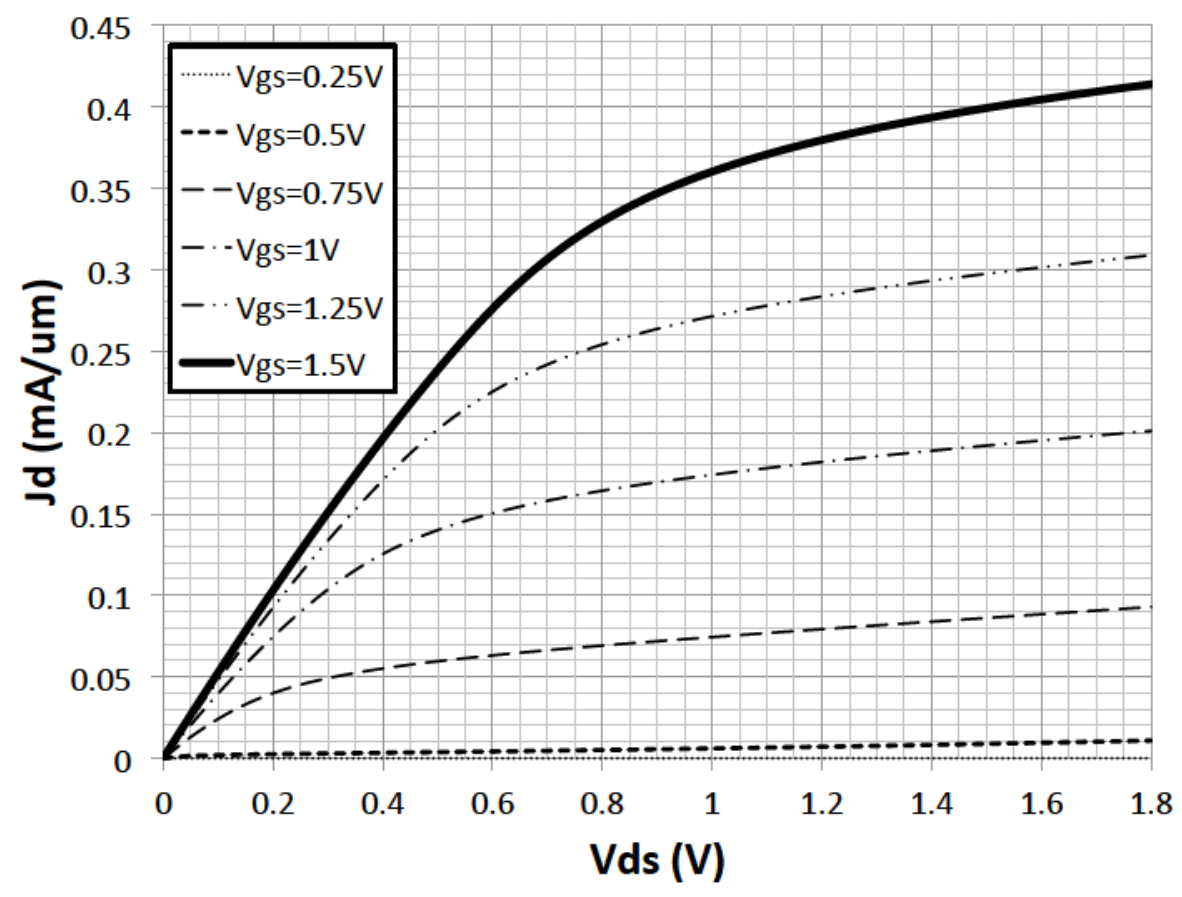

Figure 4.1: I-V curves of a 20 finger $20 \mu \mathrm{m}$ NMOS transistor in the BiCMOS process used to implement the chip

Discussion about how these are used in the design will follow in 4.3 .

\subsection{Test Chip Architecture}

Fig. 4.3 shows a system diagram of the PLL test chip. The PLL forms the main core of the test chip, with an $50 \Omega$ buffer driving the output at approximately $0 \mathrm{dBm}$. The PLL is a conventional $\Delta-\Sigma$ frequency synthesizer with an on-chip loop filter. The controls of the circuit (including charge pump current, loop divide ratio and output divide ratio, phase detector polarity, VCO frequency band, etc.) are controlled through a set of digital registers which can be accessed off-chip through the use of an inter-integrated circuit (I2C) bus. The reference clock on the chip is set through the use of an on-chip $30 \mathrm{MHz}$ crystal oscillator. The following sections will discuss in further detail the design of the parts of the test chip. There are multiple power supplies on the chip (as is typical for mixed signal circuits, digital and analog supplies are kept separate); some supplies are fed directly from a $3.3 \mathrm{~V}$ supply off chip, while others are generated through on-chip LDO regulators.

Note that since the test chip is based off of an existing production design, there are aspects of the test chip which are not optimized for chip testing. This includes numerous 


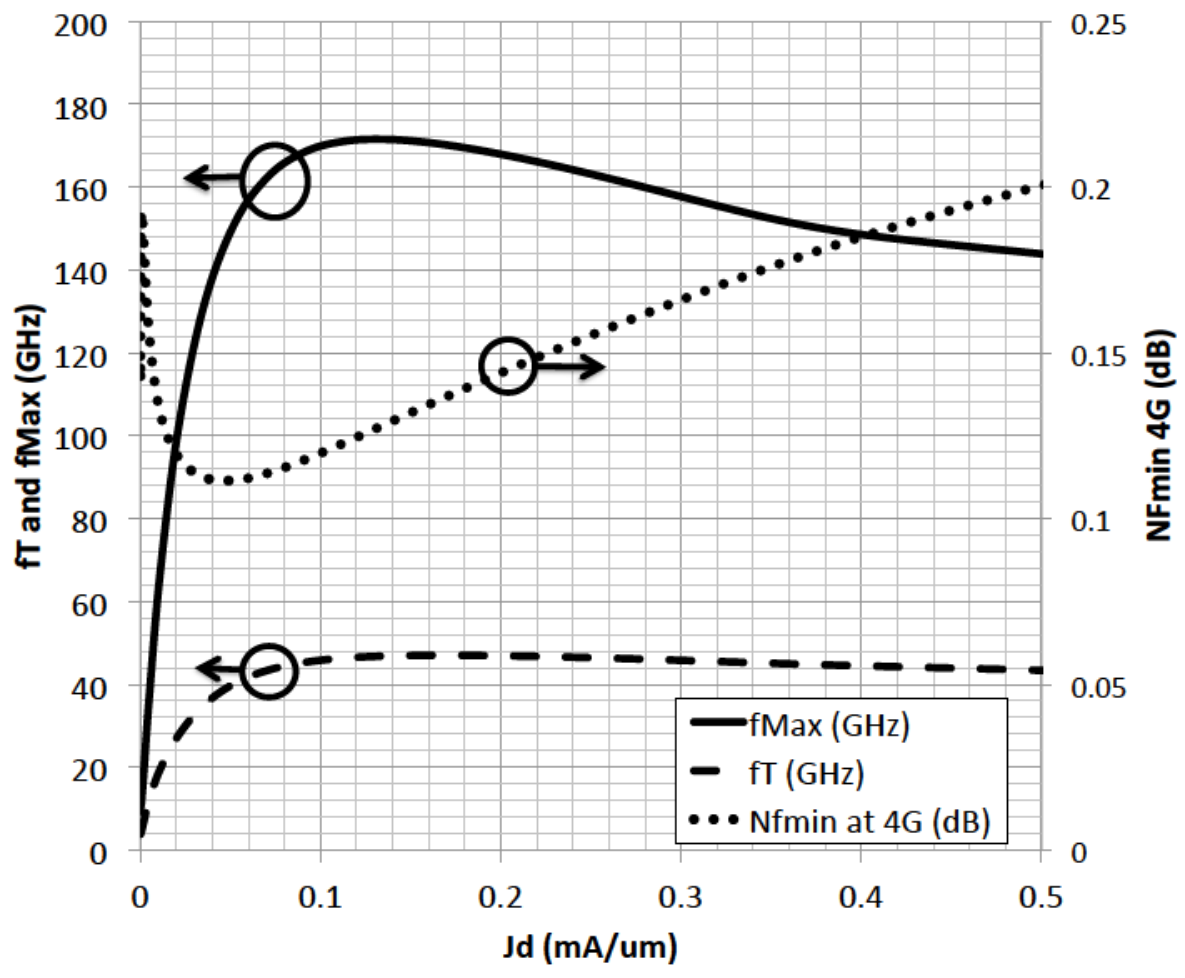

Figure 4.2: Basic simulated RF parameters of a 20 finger $20 \mu \mathrm{m}$ NMOS transistor in the BiCMOS process used to implement the chip. The large $f_{\max }$ and low NFmin values likely indicate poor modelling of gate resistance. 


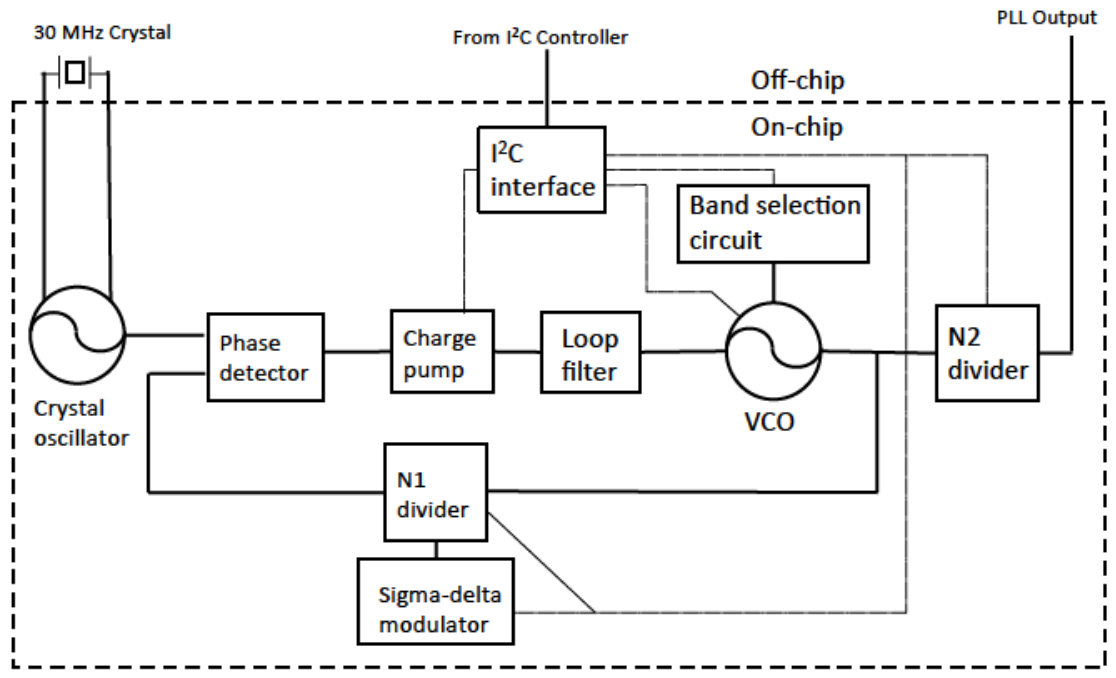

Figure 4.3: System diagram of the test chip

features which are disabled or otherwise not used, limited output pads to serve as test points, and a sub-optimal layout.

\subsection{Voltage controlled crystal oscillator}

The reference signal source for the frequency synthesizer is implemented with an on-chip voltage-controlled crystal oscillator (VCXO) using a CMOS inverter Pierce topology. The schematic of the oscillator can be seen in Fig. 4.4. A 25 to $40 \mathrm{MHz}$ off-chip quartz crystal is connected to a varactor and a series of programmable load capacitors. Resistors connected to the output can also be adjusted to allow the signal level to be varied, while the load capacitors allow for some tuning.

For the tests done for this thesis, a PCB using a $30 \mathrm{MHz}$ crystal is used. The voltage control, which can be used for tuning, or further temperature compensation, is not used. The resistors are configured to be set to the maximum output level.

\subsection{Power supply, Voltage Regulation and References}

The chip is powered using two $3.3 \mathrm{~V}$ supplies that are connected off-chip: one for the digital logic and one for the analog circuitry. Since the digital logic operates at a $1.8 \mathrm{~V}$ supply, an on- chip linear regulator is used to generate the additional supply. This also serves to reduce power supply noise coupling into the rest of the circuit. Most of the analog circuitry runs 


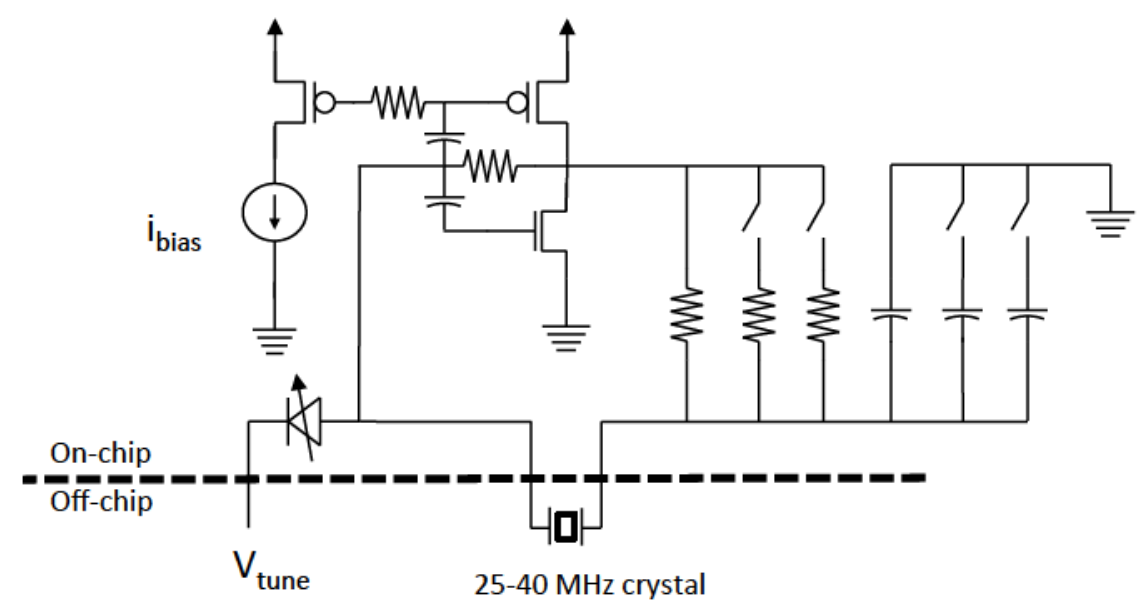

Figure 4.4: Schematic of the voltage controlled crystal oscillator used as the frequency reference on the test chip

directly from the analog supply, although the VCO uses an additional linear regulator, as it uses thinner oxide transistors and runs from a $1.8 \mathrm{~V}$ supply. All of the linear regulators and the current sources are controlled by a series of on-chip band-gap generators.

The regulator used to generate the local power supplies is a conventional low-drop out (LDO) regulator, whose schematic can be seen in Fig. 4.5. The negative feedback path and the op-amp adjusts the gate voltage on the PMOS transistor in an attempt to set the output voltage to a specified value, which is determined by the reference voltage (set by a band-gap generator) and the ratio of the resistive voltage divider. This type of regulator has poor power efficiency, since the energy lost over the voltage drop is dissipated as heat in the PMOS transistor, but has low supply noise, and minimal supply ripple. A plot of the simulated output noise can be seen in Fig. 4.6. The values shown in this plot are used in the VCO simulations to evaluate the VCO phase noise performance with the expected power supply noise.

\subsection{Divider}

The PLL uses two independent dividers: one is a feedback divider inside the loop and the other is an output divider which generates the signal fed to the output. Both dividers are programmable and independent allowing for a wide range of output frequencies. Thus, both dividers are multi-modulus, allowing for variable division ratios, and consist of higher frequency current mode logic (CML) divider stages followed by a lower frequency CMOS divider 


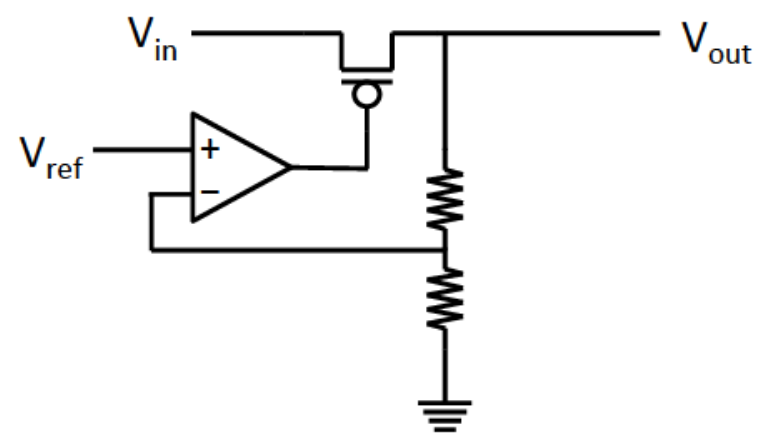

Figure 4.5: Schematic of the LDO regulator used to generate the regulated power supplies

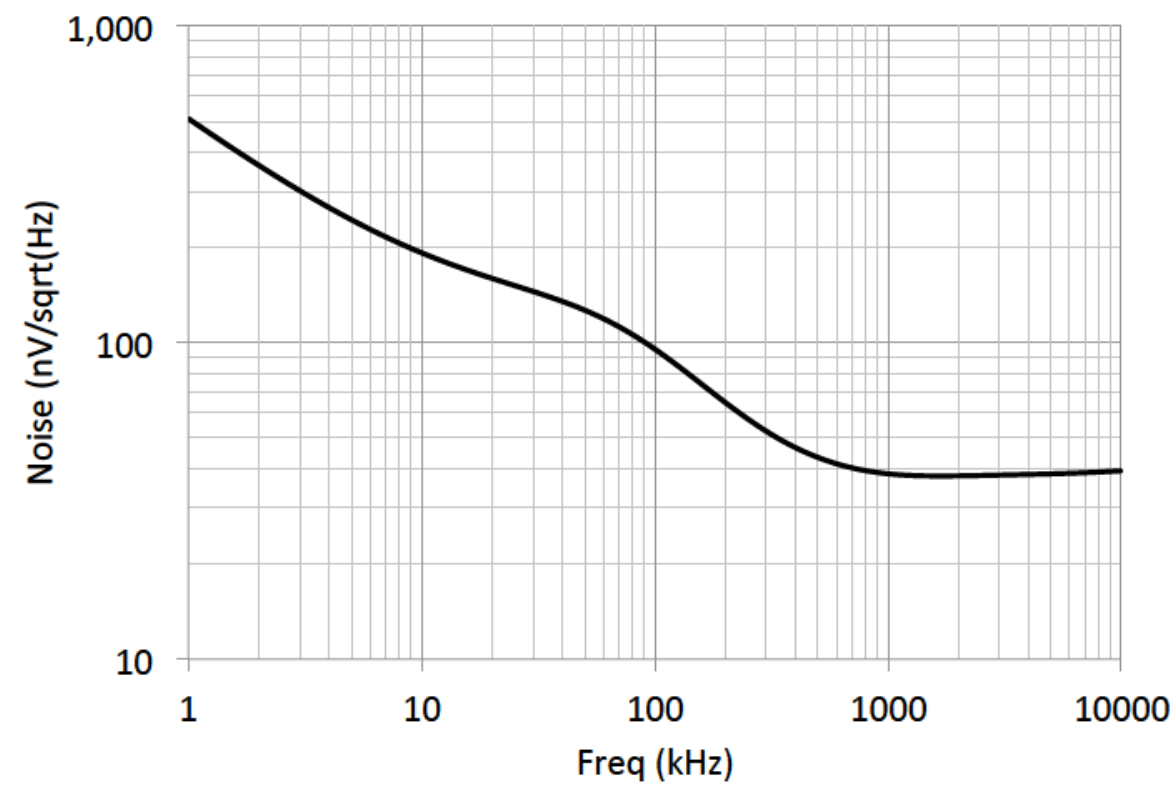

Figure 4.6: Plot of the simulated LDO output noise voltage 


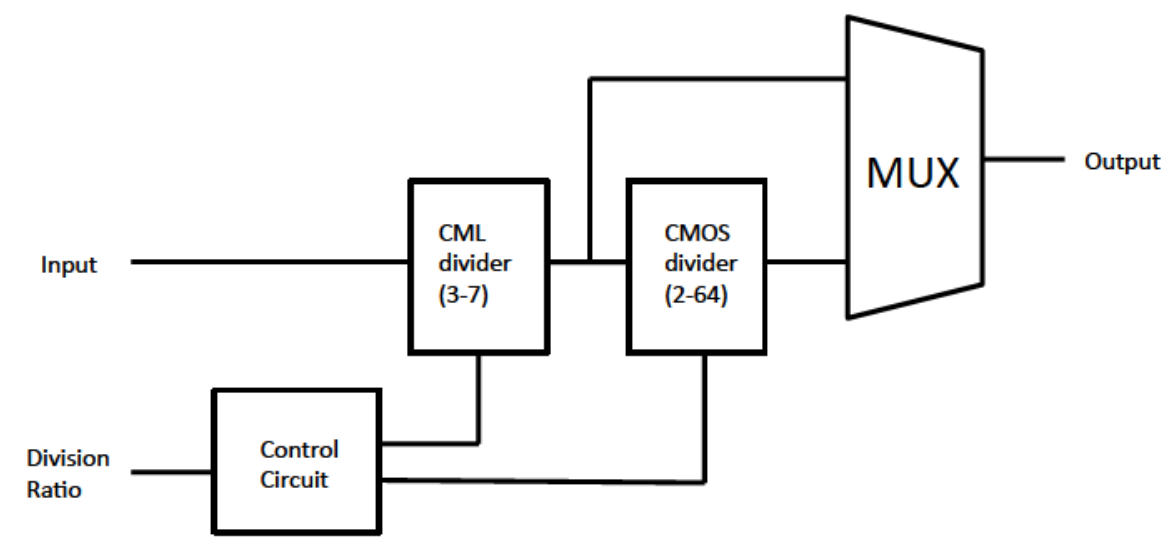

Figure 4.7: Schematic of the overall architecture of the frequency divider

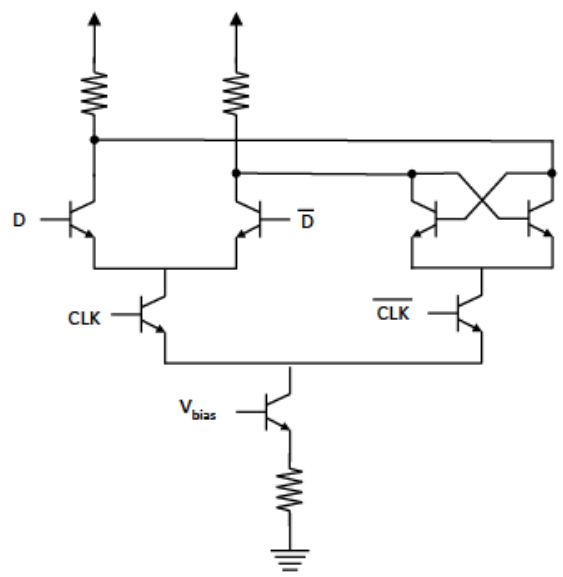

Figure 4.8: Schematic of a CML latch using bipolar transistors

using a True Single Phase Clock (TSPC) topology which uses dynamic logic with simplified logic for enhanced high speed performance. The CML dividers use the static master- slave latch configuration while the CMOS dividers use true single-phase clock (TSPC) flip-flops. The in-loop divider has a divider range of 48 to 255 , although only a limited subset of that is needed (90-140), while the output divider has a division ratio range of 3 to 320 , giving an output frequency range of $10 \mathrm{MHz}$ to $1.4 \mathrm{GHz}$. Fig. 4.7 shows the overall architecture of one of the dividers, while Fig. 4.8 shows the base schematic of the CML latch used for the CML dividers. An example TSPC divider is shown in Fig. 4.9, although the design actually used is modified from this in order to implement the multi-modulus function. The actual schematic for this circuit is not shown because it was no longer available at the time of writing. 


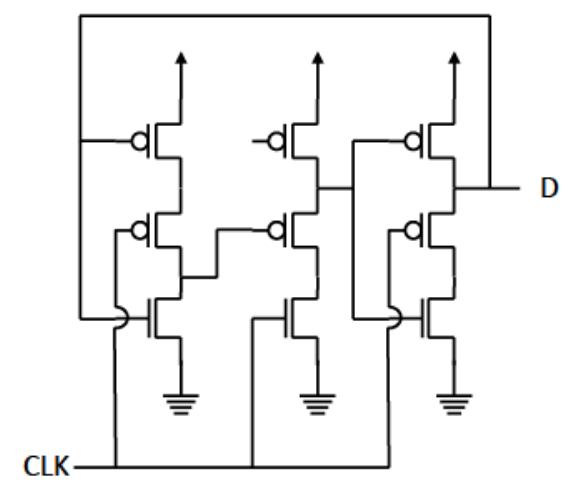

Figure 4.9: Schematic of a CMOS TSPC divider

\subsection{Phase detector and Charge pump}

The loop uses a modified version of the tri-state phase frequency detector based on two D-flip-flops and digital logic described in 2.1.4, which generates an UP and a DOWN input, which are generated based on whether the reference signal is faster or slower than the divided down output. These outputs are then fed into the charge pump. Fig. 4.10 shows a schematic of the charge pump. A reference current is mirrored on two branches, each controlled by a CMOS switch. The currents are redirected and summed at the output. A polarity control exists to allow external control over whether the UP and DOWN inputs increase or decrease the output voltage respectively or vice versa. $\mathrm{RC}$ elements are in the charge pump to filter the currents.

The charge pump is run from a $3.3 \mathrm{~V}$ supply allowing for a large output voltage which in turn can maximize tuning range per frequency band. The charge pump is capable of outputting near rail-to-rail voltages $(0$ to $3.3 \mathrm{~V})$, but its nominal operating conditions are from $0.7 \mathrm{~V}$ to $2.7 \mathrm{~V}$.

The bias current is digitally programmable to allow for 8 possible current settings from $250 \mu \mathrm{A}$ to $2 \mathrm{~mA}$.

\subsection{Loop filter}

The loop filter for the PLL is constructed on-chip, primarily with the dual layer MIM caps available in the process, but also with poly-silicon capacitors. Integrating the loop filter on-chip adds several advantages, including reduced cost of materials and assembly for the PCB, reduced packaging costs, and reduced susceptibility for noise coupling, since the loop 


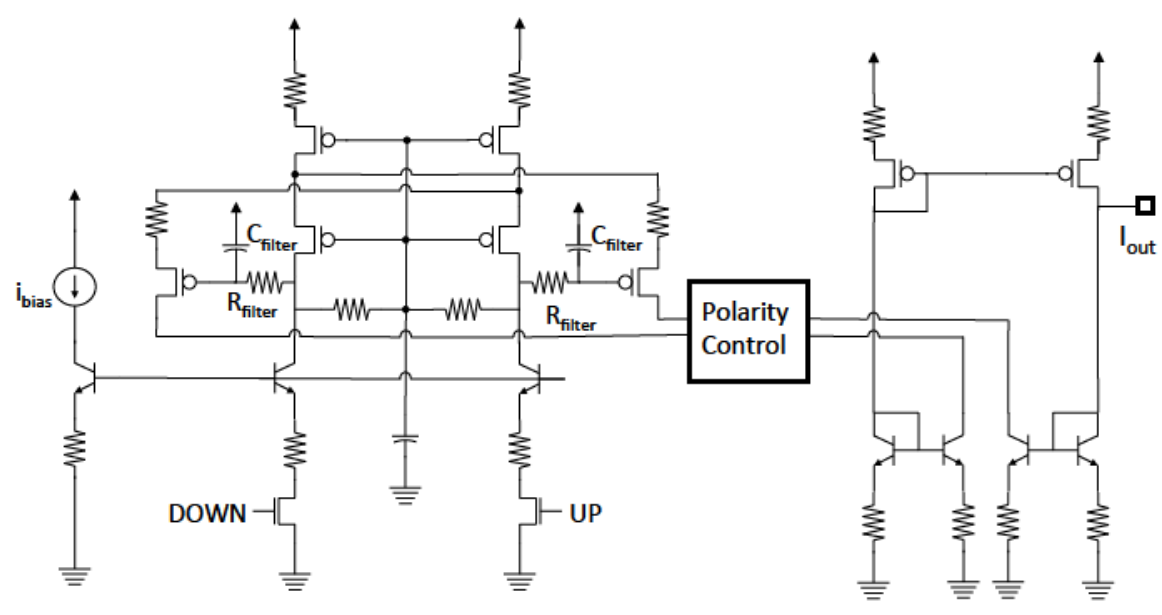

Figure 4.10: Schematic of the charge pump

filter is located close to the VCO, leaving fewer structures where noise can couple onto the sensitive VCO control voltage input. However, on-chip integration also has some drawbacks, including larger chip area used, potential yield problems due to the large area of very thin insulating nitride regions needed, and the inability to control the filter bandwidth (although the programmable charge pump current still allows control over the overall loop bandwidth). In regards to the second drawback, in the process used, there are design rules that limit the total amount of MIM capacitors, which was found to limit the size of power supply decoupling that could be implemented. Poly-silicon resistors and capacitors also have nonlinear characteristics which can cause intermodulation distortion.

\subsection{Frequency Band Switching Circuit}

A critical goal of the test chip is to demonstrate that the slow frequency band switching circuit is able to prevent cycle slipping in the PLL. Thus, the capacitor bank slow switching circuit must be able to switch slowly enough so as to limit the maximum transient phase error see when switching frequency bands to within the linear region of the PFD. The design and operation of the circuit to perform this was discussed extensively in Section 3.1.3.

Simulations of the PLL indicated that a minimum phase error reduction of $35 \%$ would be required to prevent cycle slipping when band switching, although a higher target of $90 \%$ is instead set for a larger margin of error and to further minimize the switching transients. This requires a minimum rise/fall time of $60 \mathrm{\mu s}$. Based on the results shown in 3.28, this target can be achieved under all process corners with 14 diode connected transistors with only the existing parasitic wiring capacitance. Thus, additional fixed capacitance on these 
lines is not needed.

\subsection{Voltage-Controlled Oscillator}

The PLL components described in the previous sections were used unmodified from their original version from the commercial part from which the test chip was derived. However, a new VCO was used. Although this was not necessary (since the band switching circuit could easily have been implemented in the original VCO), a new VCO was designed in order to reduce phase noise and examine several concepts in the VCO design. This section will discuss the design of the VCO in more detail.

\subsubsection{Oscillator Specifications}

Before the design of the oscillator can be discussed, the general specifications for it must be determined, including the tuning range, output requirements, phase noise, power consumption, area requirements etc.

The tuning range is partially determined by the divide ratio available to the divider, since the reference frequency is effectively fixed. With the existing divider from the original chip, the possible divide ratios ranged from 48 to 255; thus, this supports a VCO frequency range from $1440 \mathrm{MHz}$ to $7650 \mathrm{MHz}$ with a $30 \mathrm{MHz}$ crystal oscillator, although the divider would likely fail to operate with an input frequency above $4.5 \mathrm{GHz}$. An important function of the original frequency synthesizer is its wide tuning range to allow use in many applications so a wide tuning range was in turn sought for the replacement VCO. Since the divider is able to divide in integer steps starting from 3 , in order to have a continuous frequency range, the ratio between the highest and lowest VCO frequencies should be 1:1.333. To make best use of the output divider range, the frequency range should be maximized. Thus, a goal of approximately $3 \mathrm{GHz}$ to $4 \mathrm{GHz}$ was used instead.

Phase noise for a VCO usually should be kept as low as possible to improve the output jitter. A PLL attenuates jitter from the VCO below its loop bandwidth, so the main target for the VCO phase noise was for larger offsets. An initial target of $-120 \mathrm{dBc} / \mathrm{Hz}$ was set, although further reduction in noise would be beneficial.

Generally speaking, in VCO design, it is possible to reduce phase noise by admittance scaling. Essentially, this is equivalent to using multiple copies of a VCO, coupling them together and summing the outputs. The output waveform will add in the signal domain, while the noise will add in the power domain, attenuating noise by $3 \mathrm{~dB}$ per stage, but also increasing power consumption by $3 \mathrm{~dB}$. Thus, VCO designs are typically rated by a figure 
of merit with phase noise and DC power consumption:

$$
\operatorname{FoM}(\Delta \omega)=\frac{\left(\frac{\omega_{0}}{\Delta \omega}\right)^{2}}{\mathcal{L}(\Delta \omega) P_{D C[m W]}}
$$

where $\Delta \omega$ is the phase noise offset frequency, $\mathcal{L}(\Delta \omega)$ is the phase noise at that offset frequency, $\omega_{0}$ is the oscillation frequency and $P_{D C[m W]}$ is the DC power consumption in $\mathrm{mW}$. Thus, to determine how effective the VCO design was, this figure of merit was used when rating different design topologies and design variables. For CMOS and BiCMOS designs, good figures of merit are typically below $-180 \mathrm{dBc} / \mathrm{Hz}$ with the best results being below -190 .

The output of the VCO was to be fed into a set of buffers which led into the dividers. These buffers were $50 \Omega$ common base amplifiers designed to be fed by a $50 \Omega$ open collector (or open drain) buffer. The output of the divider buffer should be approximately $300 \mathrm{mV}$ in order for proper operation.

\subsubsection{Oscillator topology and Power Supply Noise}

For RF purposes, there are several competing topologies. Due to the need for low phase noise, a tuned LC oscillator was preferred over a ring oscillator design, since the resonant LC circuit has a narrow bandwidth which helps filter out noise.

For LC oscillators, the cross-coupled pair topology (sometimes referred to as the -gm topology) is a common design due to its inherently broad band characteristics, relaxed start up conditions, good phase noise characteristics, low power consumption and high output swing. As such, it is highly studied in the literature, with numerous examples available. This made it a good candidate to be used for this design. For the process used, the MOSFET transistors were found to have better overall performance than the bipolar transistors. With both NMOS and PMOS transistors available, 3 cross coupled pair combinations are possible, using NMOS, PMOS and both (called a CMOS cross coupled pair) to form the necessary cross-coupled pairs.

It has been shown in the literature that using a CMOS cross-coupled pair can provide optimal performance due to greater current efficiency and higher signal swing, as discussed in [186]. However, when power supply noise is taken into account, the output phase noise greatly increases, no longer making a CMOS cross-coupled pair optimal. In many analog circuits, using a differential topology can prevent power supply noise from appearing at the output of a circuit since it is added to both positive and negative outputs equally, leading to a low power supply rejection ratio (PSRR). However, with a VCO, this is not the dominant method for power supply noise to appear at the output, since at the VCO frequency, the $1 / f$ noise that dominates the output noise will be small, resulting in a negligible impact on the output 


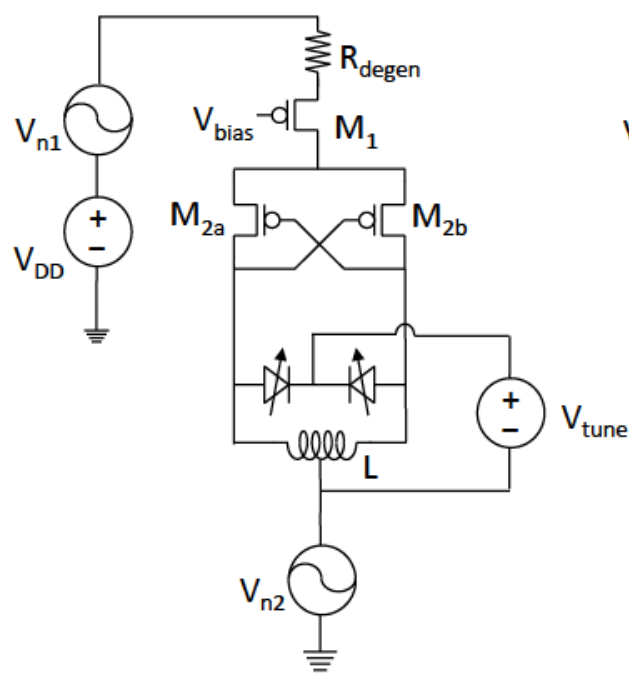

(a)

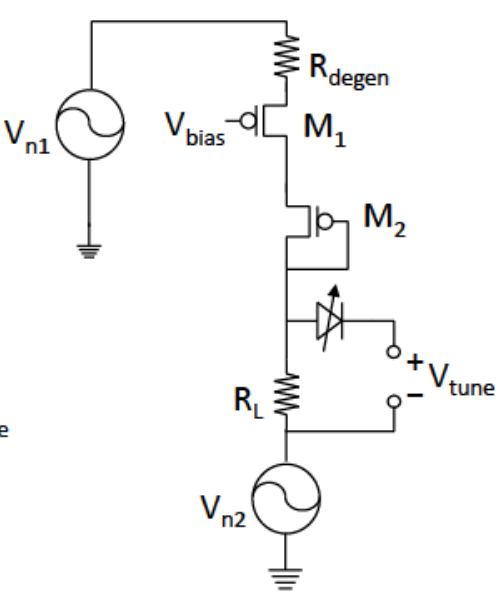

(b)

Figure 4.11: Schematic of (a) a typical PMOS cross-coupled pair VCO and (b) simplified single ended version illustrating the common mode noise path

phase noise. However, the tuning voltage is commonly single ended (to simplify the charge pump design and due to the difficulty in implementing a differential VCO tuning port), which can allow low frequency power supply noise to be upconverted as phase noise. In a CMOS cross coupled pair oscillator, the tuned resonator is at a high impedance providing minimal power supply rejection, whereas for an NMOS or PMOS cross-coupled pair oscillator, it is at a low impedance, allowing supply noise to be attenuated. While a second order harmonic trap can be added to reduce noise, this method is aimed at reducing noise folding at the second harmonic. An additional path leading to noise be directly coupled to the varactor is still present. Small signal analysis shows the mechanism behind this.

Fig. 4.11 shows a schematic of a typical PMOS cross-coupled pair oscillator in (a), with different sources for power supply noise and ground noise, while (b) shows a redrawn version showing the signal path between the noise sources and the varactor tuning port. In these schematics, $\mathrm{V}_{\mathrm{DD}}$ is the power supply, $\mathrm{V}_{\mathrm{n} 1}$ is the supply noise, $\mathrm{V}_{\mathrm{n} 2}$ is the ground noise, $\mathrm{V}_{\text {bias }}$ is the current source bias voltage, $V_{\text {tune }}$ is the tuning voltage, $R_{\text {degen }}$ is the current source degeneration resistor, $\mathrm{L}$ is the tank inductor and $\mathrm{R}_{\mathrm{L}}$ is the tank inductor's low frequency series resistance. M1 is the current source transistor, and M2a/M2b are the cross coupled pair transistors (combined as M2). The cross-coupled pair can be combined into a diode connected transistor since the noise source sees a common mode version of the circuit. The 


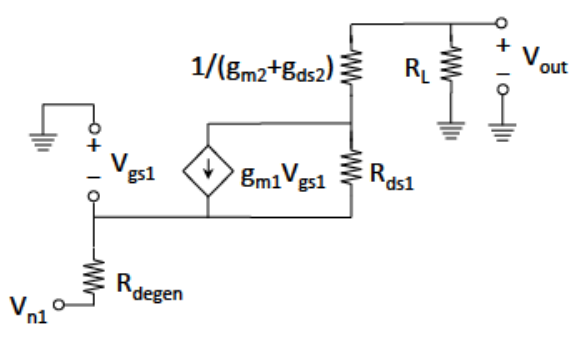

(a)

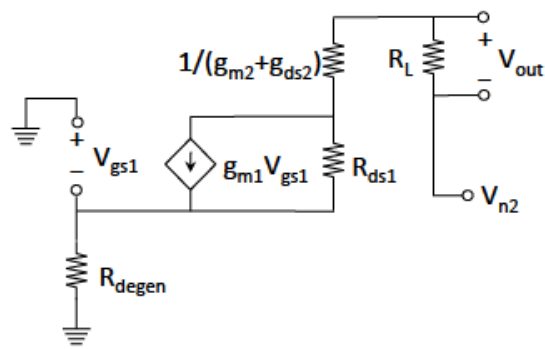

(b)

Figure 4.12: Small signal equivalent circuit of the PMOS cross-coupled pair VCO showing noise path for (a) power supply noise and (b) ground noise

varactor connection to the tank relative to the tuning port is considered the output for the noise, since it provides the most sensitive path for supply noise to be up-converted as phase noise. In a PMOS cross-coupled pair oscillator, the PLL loop filter is referenced to the same ground as the VCO, hence sharing the same noise source.

Fig. 4.12 shows the small signal equivalent models for the noise sources showing the power supply noise source in (a) and the ground noise source in (b). Conventional circuit analysis can then be used to find the transfer functions from the noise source to the varactor.

For power supply noise, the following expression can be derived:

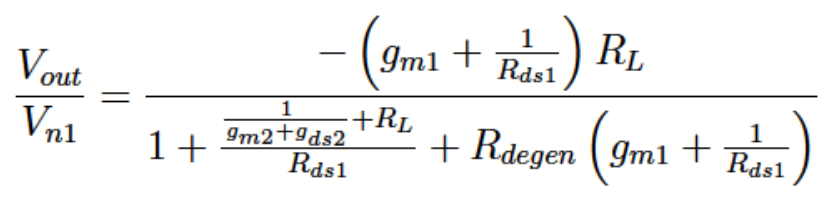

If $R_{d s 1}$ is large, the expression can be simplified:

$$
\frac{V_{\text {out }}}{V_{n 1}}=\frac{-g_{m 1} R_{L}}{1+R_{\text {degen }} g_{m 1}} \approx \frac{-R_{L}}{R_{\text {degen }}}
$$

Similarly, for the ground noise, a noise transfer function can be derived:

$$
\frac{V_{\text {out }}}{V_{n 2}}=\frac{R_{L}}{R_{d s 1}\left(1+\left(g_{m 1}+g_{d s 1}\right) R_{\text {degen }}\right)+\frac{1}{g_{m 2}+g_{d s 2}}+R_{L}}
$$

which can be simplified follows for typical values:

$$
\frac{V_{\text {out }}}{V_{n 2}}=\frac{g_{d s 1}}{g_{m 1}+g_{d s 1}} \frac{R_{L}}{R_{\text {degen }}}
$$

These expressions indicate that the noise is attenuated by a factor proportional to the ratio of the tank inductor's series resistance to the output impedance of the current source. 


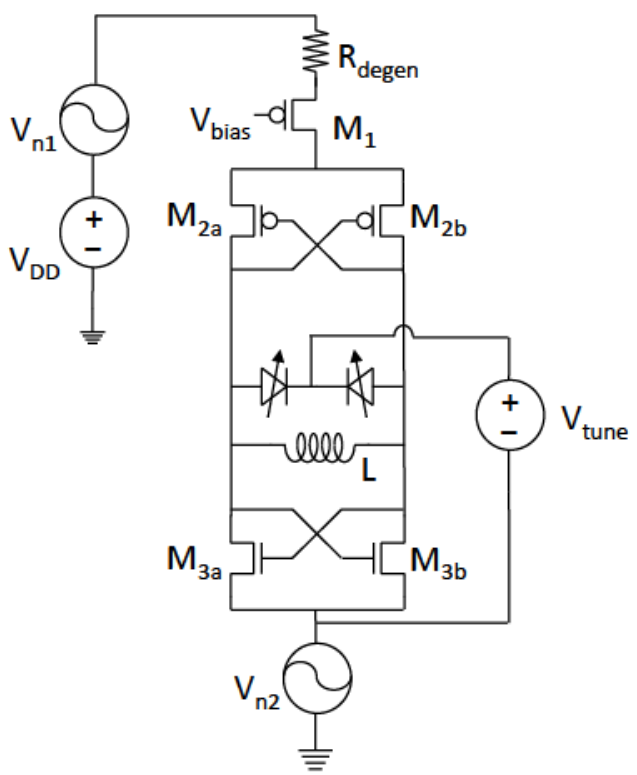

(a)

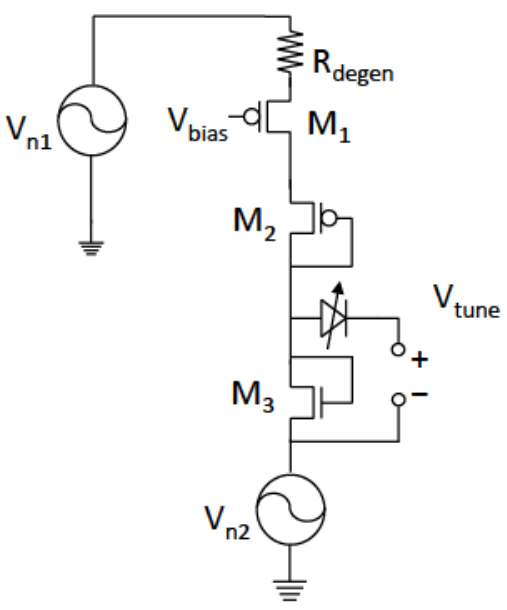

(b)

Figure 4.13: Schematic of (a) a typical CMOS cross-coupled pair VCO and (b) simplified single end version illustrating the common mode noise path

With the series resistance of a typical tank inductor well below $1 \Omega$ and typical degeneration resistors on the order of $100 \Omega$ or more, the noise can be attenuated by 40 to $60 \mathrm{~dB}$, as was verified by an analysis of noise contributors. As simulations of the oscillator show an approximate increase of $30 \mathrm{~dB}$ of phase noise when supply noise is simulated without any mitigation factors, the presence of a low impedance path allows the power supply noise contribution to be decreased to relatively negligible values.

The analysis of a NMOS cross coupled pair oscillator is similar to that of the PMOS design, since the designs are complementary, with the tuning voltage applied to the power supply instead of the ground.

The CMOS cross coupled pair design is different however, since the tank inductor no longer supplies power to the oscillator and thus no longer provides a low impedance path for the power supply noise. A schematic for a typical CMOS cross coupled pair can be seen in Fig. 4.13 (a), with the equivalent common mode version for noise analysis in (b). The small signal equivalent circuit can be seen in Fig. 4.14 (a) for power supply noise and (b) for ground noise. The noise transfer functions can be calculated as: 


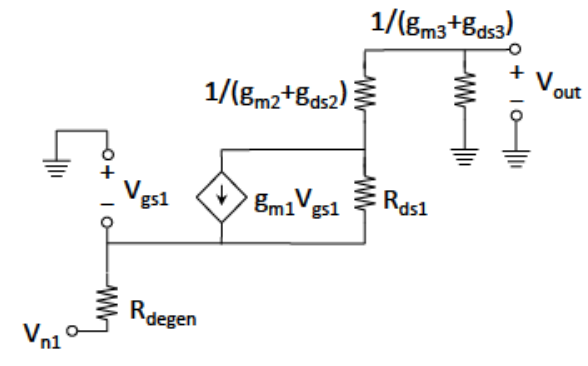

(a)

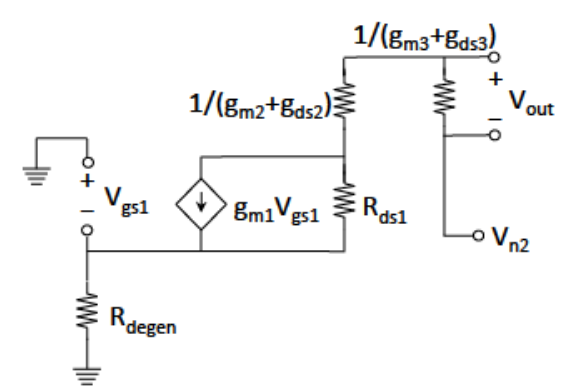

(b)

Figure 4.14: Small signal equivalent circuit of the CMOS cross-coupled pair VCO showing noise path for (a) power supply noise and (b) ground noise

$$
\begin{aligned}
\frac{V_{\text {out }}}{V_{n 1}} & =\frac{-\left(g_{m 1}+\frac{1}{R_{d s 1}}\right) \frac{1}{g_{m 3}+g_{d s 3}}}{1+\frac{g_{d s 1}}{g_{m 2}+g_{d s 2}}+\frac{g_{d s 1}}{g_{m 3}+g_{d s 3}}+R_{\text {degen }}\left(g_{m 1}+\frac{1}{R_{d s 1}}\right)} \\
& \approx \frac{-g_{m 1} \frac{1}{g_{m 3}+g_{d s 3}}}{1+R_{\text {degen }} g_{m 1}} \\
\frac{V_{\text {out }}}{V_{n 2}}= & \frac{\frac{1}{g_{m 3}+g_{d s 3}}}{R_{d s 1}\left(1+\left(g_{m 1}+g_{d s 1}\right) R_{d e g e n}\right)+\frac{1}{g_{m 2}+g_{d s 2}}+\frac{1}{g_{m 3}+g_{d s 3}}}
\end{aligned}
$$

As is evident, when comparing the expression of the CMOS cross-coupled pair oscillator to the PMOS cross-coupled pair oscillator, it can be seen that for the CMOS case, the typically low resistance value $\mathrm{R}_{\mathrm{L}}$ has been replaced by the much larger value $\frac{1}{g_{m 3}+g_{d s 3}}$. In typical designs, this results in an attenuation of around $6 \mathrm{~dB}$, which is insufficient to negate the effects of the power supply noise. Thus, with the planned on-chip linear regulators, the use of a CMOS cross coupled pair oscillator cannot be used.

The VCO design that was implemented used a PMOS cross-coupled pair, due to its lower $1 /$ f noise, at the cost of lower gm. The relatively low frequency involved meant that the additional parasitics caused by the larger transistor size needed to provide sufficient gain would not overly impact the tuning range of the oscillator.

\subsubsection{LC resonator design and Tank Inductor}

One of the most important elements of an LC VCO is its tank resonator, which essentially determines the quality factor of the entire tank. At RF frequencies, on-chip inductors can 
have low quality factors if not well designed, and large arrays of capacitor banks needed for a wide tuning range can also have low effective quality factors. Both of these need to be considered in the design of the VCO.

In [187], it is shown that the figure of merit of a VCO is largely limited to the tank quality factor which, at RF frequencies, is determined by the tank inductor. Thus, proper selection of the inductor is required in order to obtain low noise performance.

One assumption about the tank is the use of approximating the loss of the tank with a parallel shunt resistance with a constant value. This is generally a poor assumption since the losses tend to be contributed by series resistive effects which are frequency dependant. However, the use of a constant shunt value helps simplify the analysis and can be used to approximate the general behaviour of the circuit over a small frequency range. A parallel RLC circuit can be shown to have an impedance given by the following expression:

$$
Z_{R L C}=\frac{\frac{s}{C}}{s^{2}+s \frac{1}{R C}+\frac{1}{L C}}
$$

Using conventional second order system analysis, this can be shown to have a natural frequency at $\omega=1 / \sqrt{L C}$ and a quality factor given by

$$
Q=R \sqrt{\frac{C}{L}}
$$

Expression 4.10 shows that the quality factor of the resonator is improved with a more capacitive resonator rather than an inductive one to increase the ratio $C / L$, although the parallel resistance $\mathrm{R}$ should not be ignored. The $\mathrm{R}$ value is important not only in its role in determining the resonator quality factor, but also determining the oscillator's output amplitude for a given current. Because of the relationship between R and L, an arbitrarily small inductance value introduces problems of its own, as a small $\mathrm{L}$ value generally results in a smaller $\mathrm{R}$ value as well. This can be shown by an examination of the impedance of an inductor.

If a given inductor has a series inductance $L_{s}$ and a series resistance $R_{s}$, than at a given frequency, it can be shown to have an equivalent parallel resistance value given by the following expression.

$$
R_{p}=R_{s}+\frac{\omega^{2} L_{s}^{2}}{R_{s}}
$$

Even for an on-chip inductor, $R_{s}$ will be low (several $\Omega$ or lower), so for $R_{p}$ to have a substantial value (several hundred $\Omega$ or greater), the second half of expression 4.11 will need to be large. For a coil, the inductance increases proportionally to the length of metal 
squared, while, to an approximate degree, the series resistance increases proportionally to the length. Thus, $\mathrm{R}_{\mathrm{p}}$ increases on the order of length ${ }^{4}$, indicating a strong preference for large inductance values. This requires a large $\mathrm{L}_{\mathrm{s}}$ value, contradicting with the requirement for a small $L_{s}$ value for high $Q$. Thus, proper inductor sizing will be a tradeoff between these, and other effects.

For this application, an on-chip inductor is used as the resonator as it typical for many RF designs. This has some advantages and disadvantages. Off chip inductors will generally have improved performance (larger range of inductance values, higher quality factors) and be implemented using package wiring, PCB wiring or even bond-wires [188] for minimal added cost if bond pads already exist at that point in the circuit (such as input or output wires). However, they can have poor matching (particularly to components on-chip) and have poorer tolerances, and require additional design overhead since package and die codesign is increased. It is also difficult to produce small inductance values precisely using an off-chip implementation. Taking this into account with the fact that the package and die frame did not support additional bond-pads at the tank of the VCO, an on-chip inductor was used as the resonator, with care taken to maximize the quality factor.

On-chip inductors are typically made from spirals of metal formed on the metal layers in the IC process. In an RF CMOS or BiCMOS process, only the upper metal layers are used to reduce substrate coupling and to reduce resistance since the upper metal layers tend to be much thicker than the lower layers (although is benefit of using thick metal layers is partially mitigated by the increased number of vias to get to the top metal, and by skin effect, which limits the benefits gained with thick metals). By varying the size of the spirals, the number of turns in the coil, and the number of layers used, the inductance value can be controlled. However, the resistance of the metal lines adds loss to the inductor, resulting in finite quality factor. The parasitic capacitance of the inductor (to the substrate and to other coils) causes the inductor to resonate past a certain frequency, after which point it no longer behaves as an inductor.

The design of the on-chip inductor is largely a tradeoff between many of the design variables (diameter, line width, and number of turns) which affect key metrics of performance (inductance, quality factor, self-resonant frequency). For instance, increasing the line width reduces the series resistance, but can increase the parasitic capacitance and reduce the realized inductance value. In general, for a high quality factor tank inductor, large line widths with smaller number of turns to reduce the series parasitic resistance, while the outer diameter is set to obtain the required inductance value. In this way, a semi-optimal inductor can be designed.

Due to the parasitic capacitance that the inductor has to the substrate and ground 
planes, and due to the interwinding capacitance of the inductor, any on-chip inductor will resonate with itself at a particular frequency, denoted as the self-resonant frequency. As the frequency approaches this value, the $\mathrm{Q}$ will rise to a maximum value before reaching 0 at the self resonant frequency. The value and frequency of the maximum $\mathrm{Q}$ factor is highly dependant on geometry and thus susceptible to process variations. Thus, it is good design practice to avoid operating near the peak $\mathrm{Q}$ frequency of the inductor, particularly for circuits which require accurate estimates of inductor parameters.

Due to their complex structure and the nature of the equations governing their behaviour, inductors are difficult to model accurately analytically, unlike capacitors or resistors. Thus, the design of an inductor typically requires validation using simulators. Simulator complexity can vary from 2 or 2.5 dimensional simulators (ASITIC, EMX, Momentum) to full 3-dimensional analysis (HFSS). However, the typical work flow between IC design tools and EM simulators tends to be poor, and the accuracy is highly dependant on process parameters given by the manufacturer, which are not usually fully disclosed. Thus, simulations of the inductors used in the VCO rely on the kit models provided by the foundry, which are based off of measured results. This work flow has been supported by previous design experience with the foundry.

Simulations of the kit models of various inductors shows that in general, the optimal inductor geometry for a RF VCO uses a single turn with a wide line width while relying on the outer diameter to control the inductance value. To demonstrate this, Table 4.1 lists a variety of the inductors used, showing the number of the upper metal layers used for the coils (which are all connected in parallel to reduce resistance), the diameter, line width, number of turns, as well as the effective parallel resistance and inductance at $6 \mathrm{GHz}$, the quality factor at $3 \mathrm{GHz}$ and $6 \mathrm{GHz}$, the peak quality factor and the frequency at which the maximum quality factor is obtained. This characterization of inductors was initially conducted with a target VCO frequency of 3 to $6 \mathrm{GHz}$. Although the frequency range was later changed to 3 to $4 \mathrm{GHz}$, the information collected was useful in selecting the inductor. As can be seen from the table, for a high resonator quality factor, a single turn using 2 metal layers (in parallel) of a large radius should be used, as this greatly reduces the series resistance with minimal impact on the parasitic capacitance. The size of the loop is determined by the available layout area and the constraint to keep the inductor's self resonant frequency high.

These inductors offer a wide range of different resistance and inductance values. To gain better insight into what size inductance to use, the effective parallel resistance and peak $\mathrm{Q}$ frequency can be plotted against the parallel inductance, as can be seen in Fig. 4.15. To reduce phase noise, the oscillation amplitude should be maximized which, at a given current, requires the largest parallel resistance [189]. Since the tank inductor plays a very 


\begin{tabular}{|c|c|c|c|c|c|c|c|c|c|}
\hline \multicolumn{3}{|c|}{ Geometry } & \multicolumn{7}{c|}{ Simulation Inductor Parameters } \\
\hline $\begin{array}{c}\text { Metal } \\
\text { lay- } \\
\text { ers } \\
\text { used }\end{array}$ & $\begin{array}{c}\text { Diameter } \\
(\text { um) }\end{array}$ & $\begin{array}{c}\text { Line } \\
\text { width } \\
(\text { um) }\end{array}$ & $\begin{array}{c}\text { Num. } \\
\text { turns }\end{array}$ & $\begin{array}{c}\mathrm{R}_{\mathrm{p}} \text { at } \\
6 \mathrm{GHz} \\
(\Omega)\end{array}$ & $\begin{array}{c}\mathrm{L}_{\mathrm{p}} \text { at } \\
6 \mathrm{GHz} \\
(\mathrm{pH})\end{array}$ & $\begin{array}{c}\mathrm{f}_{\text {peakQ }} \\
(\mathrm{GHz})\end{array}$ & $\begin{array}{c}\text { Q } \\
\mathrm{Q}_{\text {peak }}\end{array}$ & $\begin{array}{c}\text { Q at } \\
3 \mathrm{GHz}\end{array}$ & $\begin{array}{c}\text { Q at } \\
6 \mathrm{GHz}\end{array}$ \\
\hline 1 & 250 & 25 & 1 & 270 & 378 & 16.5 & 25 & 15.5 & 18.9 \\
2 & 250 & 25 & 1 & 265 & 326 & 10.9 & 24 & 17 & 21 \\
1 & 250 & 25 & 2 & 660 & 960 & 5.5 & 18.3 & 17 & 18 \\
2 & 250 & 25 & 2 & 756 & 708 & 5.6 & 23 & 20 & 23 \\
1 & 200 & 25 & 2 & 176 & 267 & 24 & 29 & 14 & 18 \\
2 & 200 & 25 & 2 & 170 & 230 & 13 & 24 & 15 & 20 \\
1 & 300 & 25 & 1 & 370 & 500 & 12 & 22 & 17 & 20 \\
2 & 300 & 25 & 1 & 360 & 430 & 9.2 & 24 & 18 & 22 \\
2 & 300 & 25 & 2 & 1140 & 1350 & 4 & 24 & 23 & 22 \\
2 & 300 & 15 & 2 & 1630 & 1850 & 3.6 & 26 & 25 & 23 \\
2 & 300 & 7 & 2 & 2070 & 2600 & 4.1 & 23 & 22 & 21 \\
2 & 200 & 15 & 2 & 230 & 301 & 15 & 27 & 16 & 21 \\
2 & 200 & 7 & 2 & 290 & 400 & 18.6 & 26 & 14 & 19 \\
2 & 300 & 15 & 1 & 450 & 656 & 10.5 & 24.55 & 18 & 22 \\
\hline
\end{tabular}

Table 4.1: Comparison of simulated inductor parameters using IBM kit models

important role in the performance of the $\mathrm{VCO}$, the operating frequency should be kept well below the peak $\mathrm{Q}$ frequency if possible, since the performance around this frequency is very sensitive to process variation. An additional constraint is placed due to the design target of a large frequency tuning range using capacitor banks. Using large inductance values makes the design difficult, since the highest frequency bands requires very small capacitance. This then requires a large capacitance range with very little fixed parasitic capacitance. Small inductor values relaxes this requirement considerably.

Thus, the inductor actually used for the VCO tank is shown on the last row of Table 4.1, using a radius of $300 \mu \mathrm{m}$ with a single turn and a metal width of $15 \mu \mathrm{m}$ on the two upper metal layers. This was found to be a good compromise between quality factor, high parallel resistance, and high peak quality factor frequency. The table does not show self resonant frequency (which as found to be approximately twice the peak $\mathrm{Q}$ frequency) since it was decided to operate well below the peak $\mathrm{Q}$ frequency to minimize possible process variability, thus keeping the operating frequency well below the self resonant frequency as well. While knowledge of the self resonant frequency would be useful, it was not possible to simulate this at the time of writing.

A plot of this inductors characteristics can be seen in Fig. 4.16, which were obtained using s-parameter simulations of the kit model of the inductor. This method of simulation 


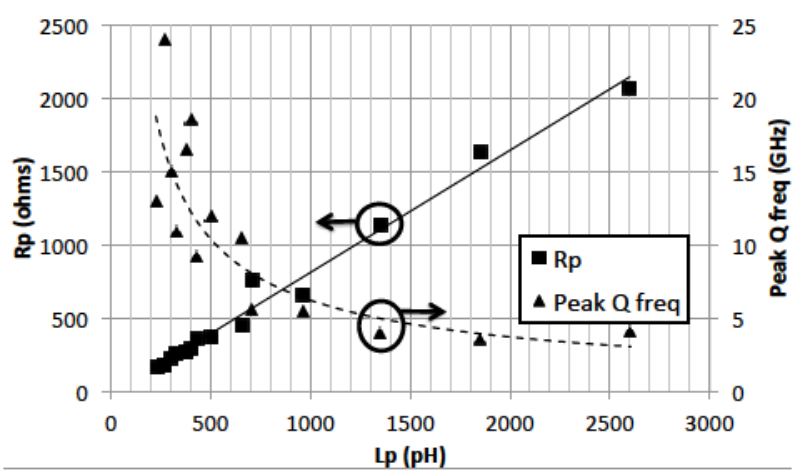

Figure 4.15: Plot comparing simulated inductor parameters for different inductor geometries

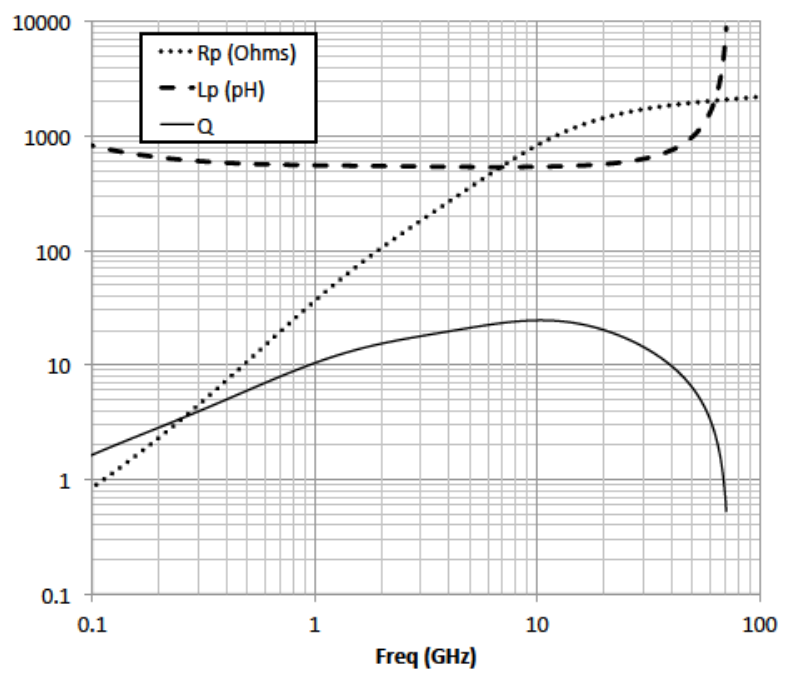

Figure 4.16: Plot of the simulated inductor parameters for the VCO tank inductor

results in poorer accuracy for the low frequency values (particularly below $100 \mathrm{MHz}$ ) since impedance will be very close to a short circuit; in addition, the assumption of a resistor in parallel with an ideal inductor is also likely to be inaccurate at low frequencies. The plot shows the values shown in Table 4.1. However, due to the low transit frequency of the transistors, the accuracy of the models used here are likely to be poor above $20 \mathrm{GHz}$.

\subsubsection{Capacitor bank and varactors}

Due to its role in controlling the frequency tuning characteristics of the VCO and its role in determining the VCO's frequency sub-bands, the design of the capacitor bank and the varactor circuity is critical to the performance of the proposed test chip. While some of the information regarding capacitor banks has already been discussed in section 3.1.1, this 


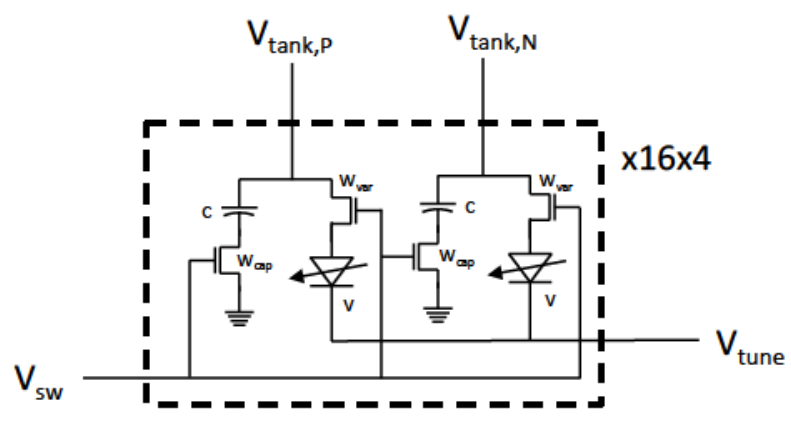

Figure 4.17: Schematic of the capacitor bank circuit

\begin{tabular}{|c|c|c|c|c|}
\hline \multirow{2}{*}{ Bank } & \multicolumn{4}{|c|}{ Component } \\
\cline { 2 - 5 } & $\mathrm{C}$ & $\mathrm{V}$ & $W_{\text {cap }}$ & $W_{\text {var }}$ \\
\hline A & $6.8 \mu \mathrm{m} \times 6.0 \mu \mathrm{m}$ & $1 \times 1.0 \mu \mathrm{m} \times 1.93 \mu \mathrm{m}$ & $4 \times 5.3 \mu \mathrm{m}$ & $4 \times 1.35 \mu$ \\
B & $7.4 \mu \mathrm{m} \times 6.0 \mu \mathrm{m}$ & $1 \times 1.0 \mu \mathrm{m} \times 2.20 \mu \mathrm{m}$ & $4 \times 6.0 \mu \mathrm{m}$ & $4 \times 1.50 \mu$ \\
$\mathrm{C}$ & $8.0 \mu \mathrm{m} \times 7.5 \mu \mathrm{m}$ & $1 \times 1.3 \mu \mathrm{m} \times 2.20 \mu \mathrm{m}$ & $5 \times 6.7 \mu \mathrm{m}$ & $4 \times 2.10 \mu$ \\
$\mathrm{D}$ & $9.0 \mu \mathrm{m} \times 9.0 \mu \mathrm{m}$ & $1 \times 1.7 \mu \mathrm{m} \times 2.30 \mu \mathrm{m}$ & $5 \times 9.0 \mu \mathrm{m}$ & $6 \times 1.90 \mu$ \\
\hline
\end{tabular}

Table 4.2: Size of component values used in the VCO capacitor banks

was mainly done from the perspective of the slow band switching circuit from a theoretical point of view. The discussion here will focus on the performance of the VCO with greater emphasis on the circuitry itself.

The design of the capacitor bank circuit used for the VCO used to demonstrate this concept is shown in Fig. 4.17. The circuit consists of 64 capacitor banks (4 groups of 16) to allow a large tuning range of approximately $2.8 \mathrm{GHz}$ to $4.2 \mathrm{GHz}$ while still maintaining $K_{V C O}$ at approximately $150 \mathrm{MHz} / \mathrm{V}$, which was required for the in-band loop components, which were already designed for this value of $K_{V C O}$. The large number of capacitor banks also allowed significant overlap between adjacent frequency bands, allowing the PLL to maintain lock over a broad temperature range without the need to switch frequency bands. Although the circuit proposed here alleviates the need for such overlap, this was done in the prototype test chip to explore the tradeoffs behind a conventional VCO capacitor bank design.

The component values of the schematic are shown in Table 4.2. The 64 capacitor banks are divided into 4 groups of 16 identical capacitor banks, labelled A, B, C, and D. The need for different capacitor banks is required to maintain an equal frequency spacing between each frequency band. This is because the frequency of a VCO depends largely on the resonant frequency of the LC tank: 

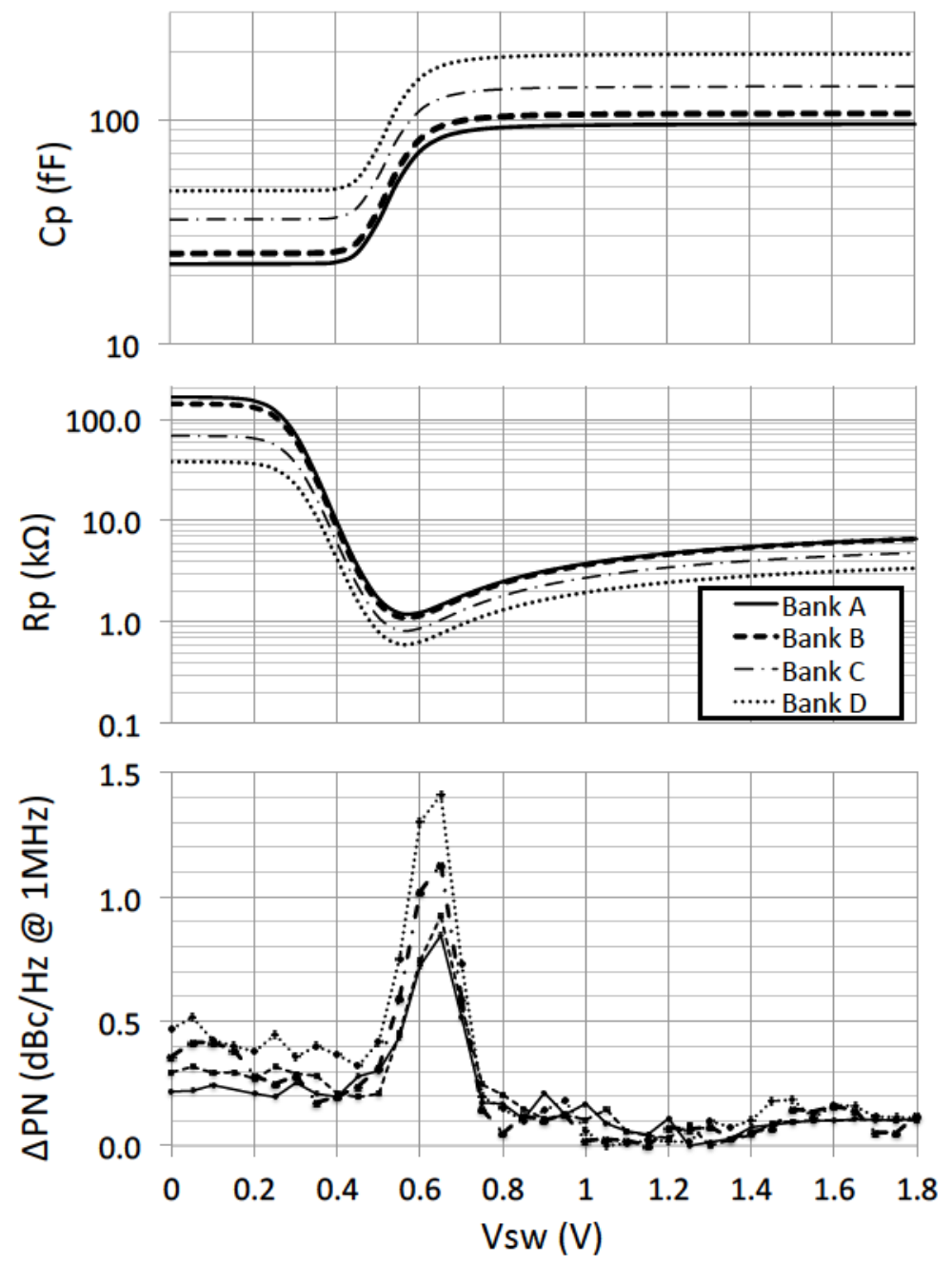

Figure 4.18: Plot of the simulated equivalent parallel capacitance and parasitic resistance (at $4 \mathrm{GHz}$ ) of the VCO capacitor banks over intermediate switch voltages 


$$
f_{\text {Tank }}=\frac{1}{\sqrt{L C}}
$$

When differentiated with respect to $\mathrm{C}$, this gives

$$
\frac{\partial f_{\text {Tank }}}{\partial C}=\frac{-1}{2 \sqrt{L C^{3}}}
$$

Thus, as $\mathrm{C}$ increases (reducing the frequency), the amount of capacitance needed to change the frequency by the same amount increases. For the frequency range used here, the use of 4 banks was found to be sufficient to keep approximately equal frequency steps. Similarly, as the frequency changes, the $K_{V C O}$ will also change. To compensate for this, each capacitor bank also features additional varactors which are connected to the main tuning voltage. The transistor switches were sized to find a balanced tradeoff between on resistance, off capacitance, and layout area.

A plot of the simulated equivalent parallel capacitance and parallel resistance (at $4 \mathrm{GHz}$ ) as the switch voltage is changed from 0 to $1.8 \mathrm{~V}$ is shown in Fig. 4.18. This plot also shows the simulated change in phase noise of the $\mathrm{VCO}$ at intermediate values of the switch voltage. When $V_{s w}$ is between $0.4 \mathrm{~V}$ and $0.8 \mathrm{~V}, C_{P}$ shows a continuous, monotonic change between lower and higher values. Thus, slow changes in $V_{s w}$ will result in gradual changes in frequency. As predicted earlier in this section, in the transitionary region, the tank $\mathrm{Q}$ drops, resulting in increased phase noise. However, due to the small size of the capacitance added in each capacitor bank, the total phase noise degradation is fairly minimal.

For continuous tuning, a hyper-abrupt junction diode varactor is used. This is used over AMOS varactors because of its ability to handle large tuning voltages, giving it a larger tuning range. This is important since the charge pump operates from a $3.3 \mathrm{~V}$ supply, allowing the tuning voltage to vary from $0 \mathrm{~V}$ to $3.3 \mathrm{~V}$. The impedance vs. tuning voltage characteristic of this type of device can be seen in Fig. 4.19.

When combined with series switches, the capacitor banks of a VCO can significantly reduce the quality factor of the resonator, especially when used in large numbers. Larger switch sizes can reduce the effect of this, but because of the added parasitic capacitance, larger switches also lower the reactance range. Thus, a tradeoff must be made between the switch size relative to the capacitor size. The use of a small inductor allows a larger tolerance for capacitive parasitics, allowing the switch sizes to be maximized while still maintaining the large tuning range. A separate fixed capacitance is avoided so as to allow for larger switch sizes. This can increase frequency range variability to due process variation, but the large tuning range of the VCO helps prevent this from causing any significant problems. 


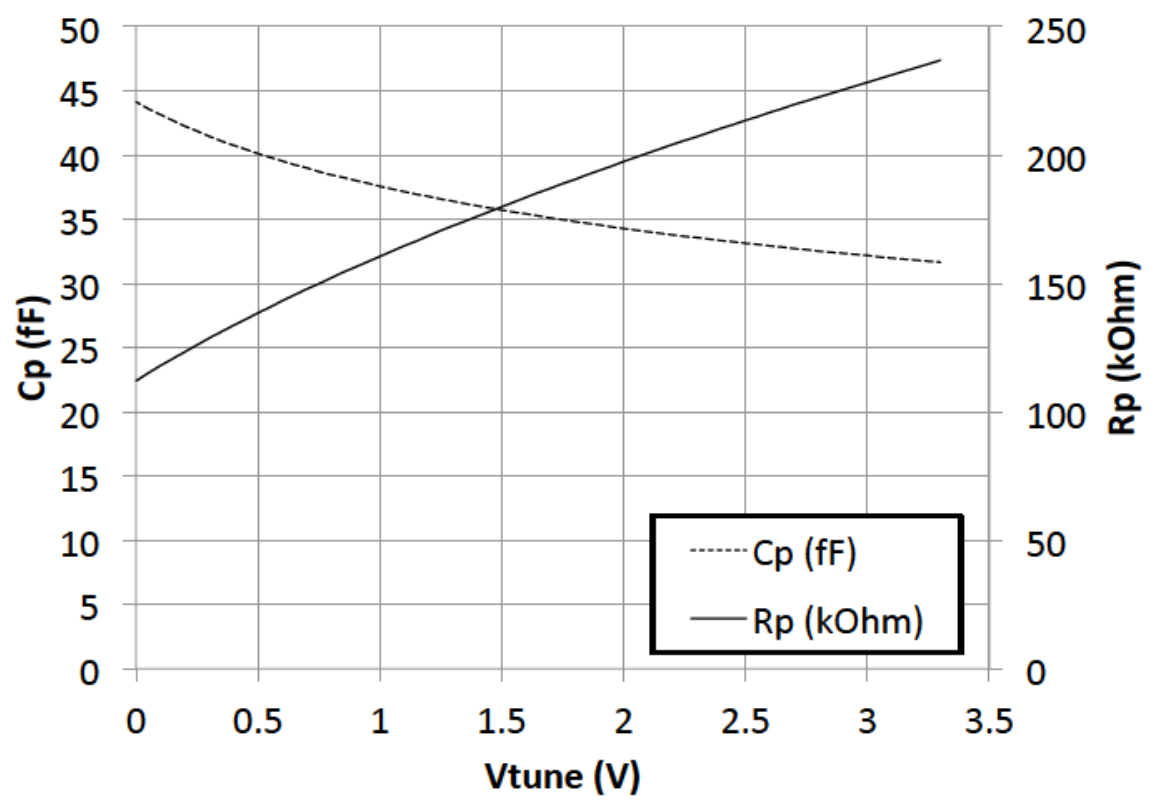

Figure 4.19: Schematic of the VCO

\subsubsection{Gain circuitry and Bias circuitry}

As was discussed in section 4.10.2, a PMOS cross-coupled pair was used for the gain circuitry to recover the signal loss in the LC resonator. The proper design of this circuit requires optimizing for noise and power efficiency across the operating frequency. The cross-coupled pair acts as a small signal negative impedance when viewed differentially. The value of the negative impedance is the negative value of the pair's transconductance. In order to create conditions to oscillate, the parallel combination of the negative conductance and the tank losses must be negative. This specifies minimum requirements for the size and bias current of the transistor. One objective is to maintain an optimal current density through the gain transistors. As shown in [190] and verified through simulations, the noise and gain properties of a transistor are at an optimal value for a particular current density, and deviations from this current density can degrade performance.

The wide frequency range of the oscillator also affects the optimal biasing of the oscillator, since the quality factor of the oscillator varies with each frequency band. As shown in expression (4.10), the quality factor increases as the resonator becomes more capacitive, but decreases as the effective parallel resistance of the resonator decreases. In addition, the quality factor of a capacitor tends to decrease at higher frequencies while that of an inductor increases at higher frequencies. In addition capacitance is added to decrease the resonant frequency with the use of series switches, which have their own parasitic loss. While these 
factors all contribute to the overall variation of the quality factor over frequency range, in a well-designed LC resonator, the quality factor of the inductor is the limiting factor of the quality factor of the entire resonator. Thus, the decrease in $\mathrm{Q}$ at lower frequencies (due to the decrease in the inductor series impedance) increases the required transconductance needed for oscillation.

The increased loss also affects the optimal transconductance value required. For an optimal design, the VCO should be biased between where the oscillation amplitude is current limited and where it is voltage limited. In the current limited domain, the oscillation amplitude can be increased with higher current levels, reducing phase noise and improving power efficiency. However, when the output voltage amplitude becomes large enough, the large signal effects of the transistor begin to dominate, which limits the output waveform. Further increases in current do not increase the oscillation amplitude, but instead increases the power consumption and the drain channel noise added. The optimal current value is highly dependant on the tank effective parallel resistance, and so scales up (similar to the minimum transconductance value needed for oscillation start-up) as the frequency of oscillation decreases.

The change in optimal current and gain settings can be handled in multiple ways. The possibility is to optimize for one particular frequency, either a central frequency to optimize for the entire range at once, or a particular frequency, if it is used more frequently or is associated with more stringent performance requirements, and then accepting the sub-optimal behaviour at other frequencies. This leads to a simpler circuit with fewer parasitics, but also leads to poorer overall performance and possibly higher vulnerability to process variation, but can be suitable for narrowband applications. An alternative is the use of variable current. By adjusting the current in the oscillator current mirror, the bias current can be increased and decreased as needed. This can be controlled automatically by monitoring the output amplitude to improve performance at different process corners or operating regions without calibration. However, because the gain transistors are kept the same size, the optimal current density is seen only at one frequency.

The alternative used for the test chip is similar to the approach shown in [184], where the gain and current is controlled by switching in cross-coupled pairs as needed, effectively changing the size of the gain transistors as more or less transconductance and current is needed. The tradeoff for this is the need for the additional switchable pairs, which add additional parasitic capacitance and resistance. In addition, the switchable pair approach results in discrete changes in transconductance and current rather than continuous, meaning that it is less able to achieve optimal performance for small changes in frequency, or process variations. Despite this, however, simulations conducted shows that the advantages of this 
approach out weigh its drawbacks.

The implementation shown in [184] places series transistors as switches in series with the drains of the cross-coupled pairs, resulting in additional series resistance between the cross-coupled pair. While this adds less parasitic capacitance, this effectively decreases the quality factor of the resonator. Thus, a different approach is pursued by placing the switches in series with the degeneration resistor of the current sources. Because it is in series with a poly-silicon resistor, the series resistance of the switch can be compensated for, and because of the presence of the current source transistor, the parasitic capacitance of the switch does not affect the resonator. Conversely, all of the cross-coupled pairs are directly connected to the tank, which results in parasitic capacitance, although the direct connections keeps parasitic resistance low. To minimize the effects of the added parasitics, the number of switchable cross-coupled pairs is limited to 2, allowing 4 different current/gain settings.

Tail inductors were used to filter out noise, as shown in [191]. These inductors serve to filter out current tail noise but also serve to resonate out the second harmonic, reducing noise that can be up-converted as phase noise. In order to implement this with the switched crosscoupled pairs, each set of cross-coupled pairs requires a separate inductor. While this requires more area, the additional area required is minimal since the quality factor requirements for these inductors is relaxed.

Some tradeoffs are needed to be made when sizing the transistors for the cross coupled pairs. Larger transistor gate lengths (accompanied by larger gate widths) have the benefit of producing lower noise and having higher output impedance, at the expense of larger parasitic capacitance. Because of the large frequency tuning range desired, smaller transistors are used with the minimum gate length. Simulations showed that if larger transistor sizes were used, the capacitors in the capacitor bank and the varactors would need to be made larger, decreasing the overall oscillator performance. For a similar reason, thin oxide, lower voltage transistors (operating from a maximum nominal supply voltage of $1.8 \mathrm{~V}$ ) were used. Thicker oxide transistors could operate from a higher supply voltage, allowing for a larger signal swing, but this would require larger transistor sizes with increased parasitic capacitance, negating the benefits that would otherwise be achieved.

The complete schematic of the VCO can be seen in Fig. 4.20. Fig. 4.21 shows the schematic of the current mirror circuit used to bias the VCO, the VCO buffer and the CML driver feeding the transmission line connected to the divider. A $75 \mu \mathrm{A}$ reference current is generated by a band-gap generator and feeds a set of current multipliers needed to generate the necessary currents.

A large current multiplication ratio is required to generate the proper bias currents from the reference current output of the band-gap generator due to the small size of the reference 


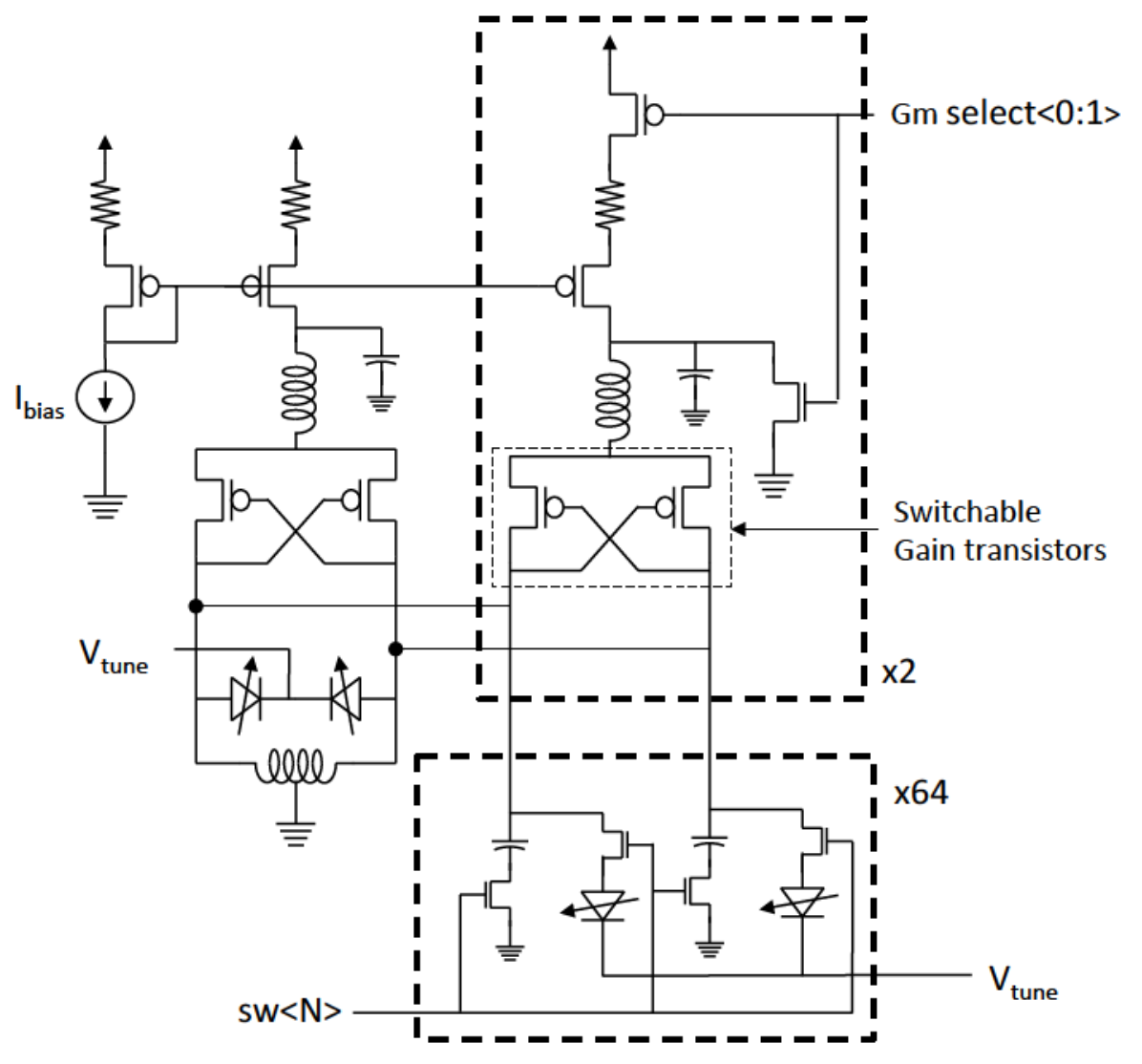

Figure 4.20: Schematic of the VCO

current. This also greatly magnifies any noise appearing on this current, so a low pass filter is placed on the bias lines of all of the current mirrors to reduce this noise. This is done with a low-pass $\mathrm{RC}$ filter using large poly-silicon capacitors, with the metal wiring and the poly-silicon resistance to act as the resistance. Physical resistors are not added since it was found in simulation that the resistors acted as an additional noise source of thermal noise.

\subsubsection{Output buffer}

To prevent the VCO from being heavily loaded (which would impair performance), output buffers are commonly used at the output of the VCO. This also allows flexibility in the design of the rest of the system (since the loading does not play a critical role) and the placement of the VCO. Due to practical concerns, the VCO was placed far away from the divider loads. Thus, a 2 stage set of buffers and amplifiers is used.

The schematic of the first buffer can be seen in Fig. 4.22. A PMOS source follower is used for several reasons: its ability to operate with a low common mode input, since output 


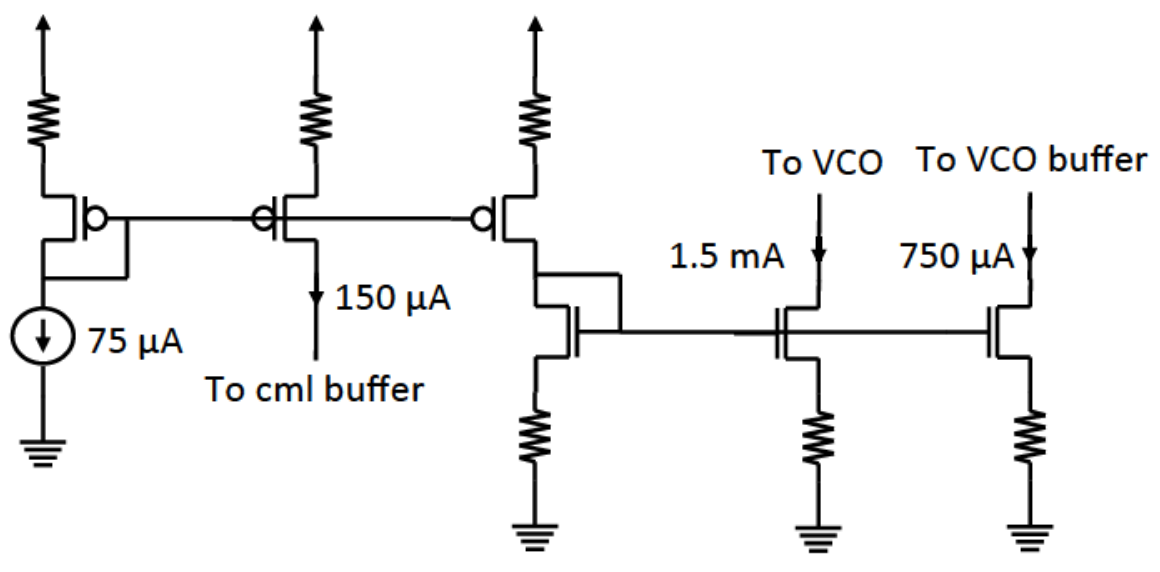

Figure 4.21: Schematic of the current mirrors used to bias the VCO, the VCO buffer and the CML driver

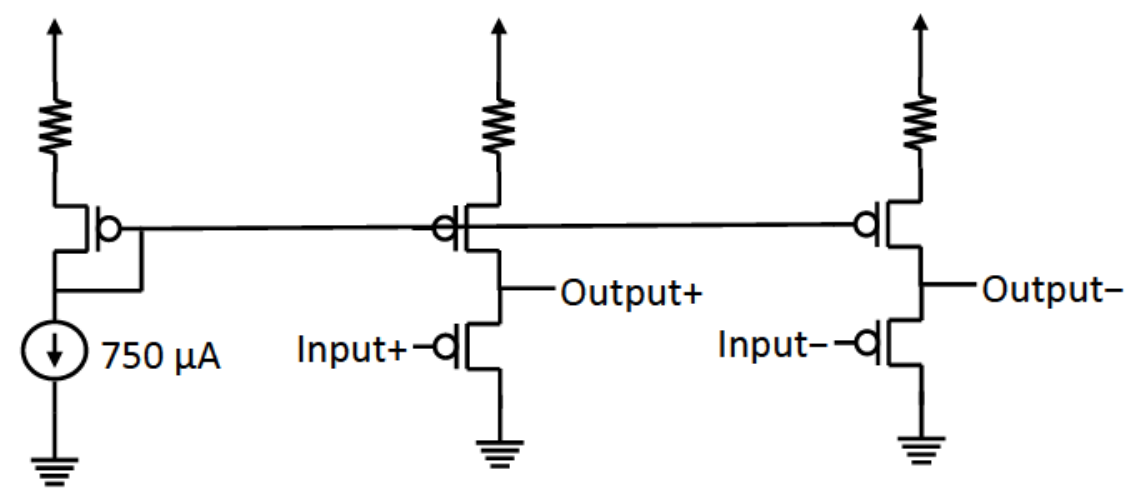

Figure 4.22: Schematic of the PMOS source follower used as the VCO buffer

tank voltage from the $\mathrm{VCO}$ has a common mode voltage of $0 \mathrm{~V}$, its ability to drive a large capacitive load while presenting a much smaller load to the VCO, and its minimal effect on phase noise.

The PMOS source follower drives a current-mode logic (CML) open drain driver, whose schematic can be seen in Fig .4.23. This driver feeds a $50 \Omega$ transmission line leading to the divider. At the divider, a common-base amplifier (with an input impedance of $50 \Omega$ ) drives a resistive load which drives the CML divider stages. 


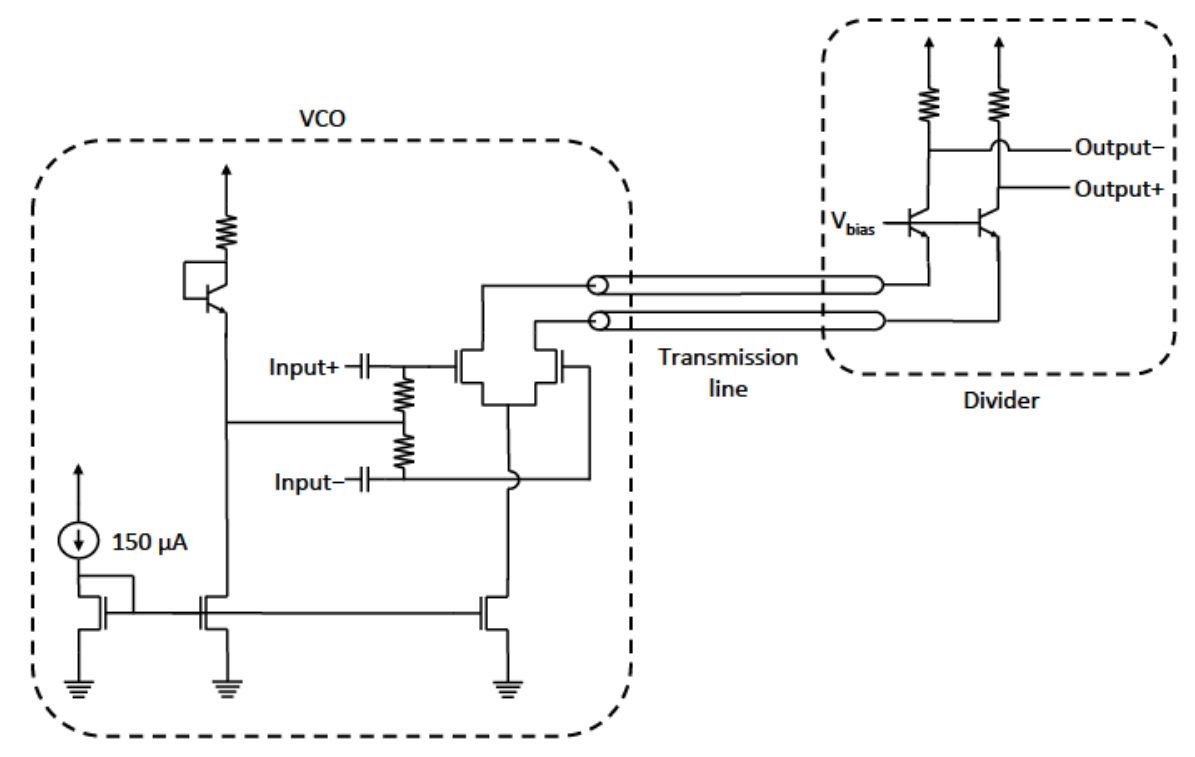

Figure 4.23: Schematic of the CML driver circuit and divider input amplifier used to connect VCO and divider circuit blocks.

\subsubsection{VCO Simulation Results}

As was the rest of the test chip, the VCO was designed using the Cadence Virtuoso set of analog IC design tools. Simulations were conducted using the Cadence Spectre simulation, primarily using periodic steady-state analysis (PSS) and periodic noise analysis to determine the VCO behaviour, including oscillation frequency, output amplitude, and phase noise. Due to licensing issues at the time of the design of the test chip, extraction tools were not available. To compensate for this, the tank parasitic capacitance was manually estimated to be $1 \mathrm{pF}$ based on measured results of previous test chips, and the oscillator was designed with some margin to compensate for unanticipated losses. Some of the results of this can be seen in the simulation results, as will be discussed.

Fig. $4.24,4.25,4.26$, and 4.27 shows the frequency vs. frequency band code settings with high and low control voltages at different current settings (GM0 being the lowest current setting and GM3 being the highest). This is done at 3 different temperatures: $-40^{\circ} \mathrm{C}$ (cold), $25^{\circ} \mathrm{C}$ (cold), and $85^{\circ} \mathrm{C}$ (hot). As can be seen, in all cases, each frequency band has enough overlap with the adjacent bands so as to cover the entire frequency range at any given frequency. Although the proposed slow band switching circuit allows this to be avoided, the prototype VCO was intentionally designed with more overlap than needed to explore the performance tradeoffs this entailed.

Fig 4.28 and 4.29 show the simulated phase noise at a $1 \mathrm{MHz}$ offset and the phase noise- 


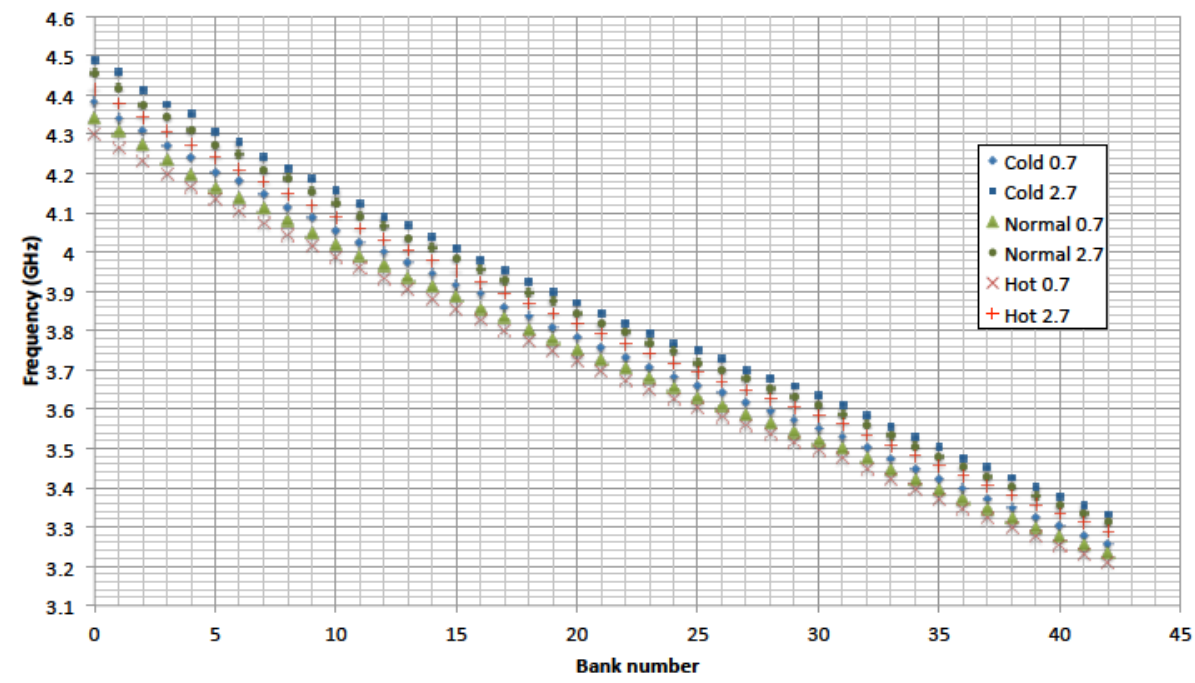

Figure 4.24: Plot of the simulated frequency vs frequency band code setting at high and low control voltages at hot, nominal and cold temperatures with the lowest current setting GM0

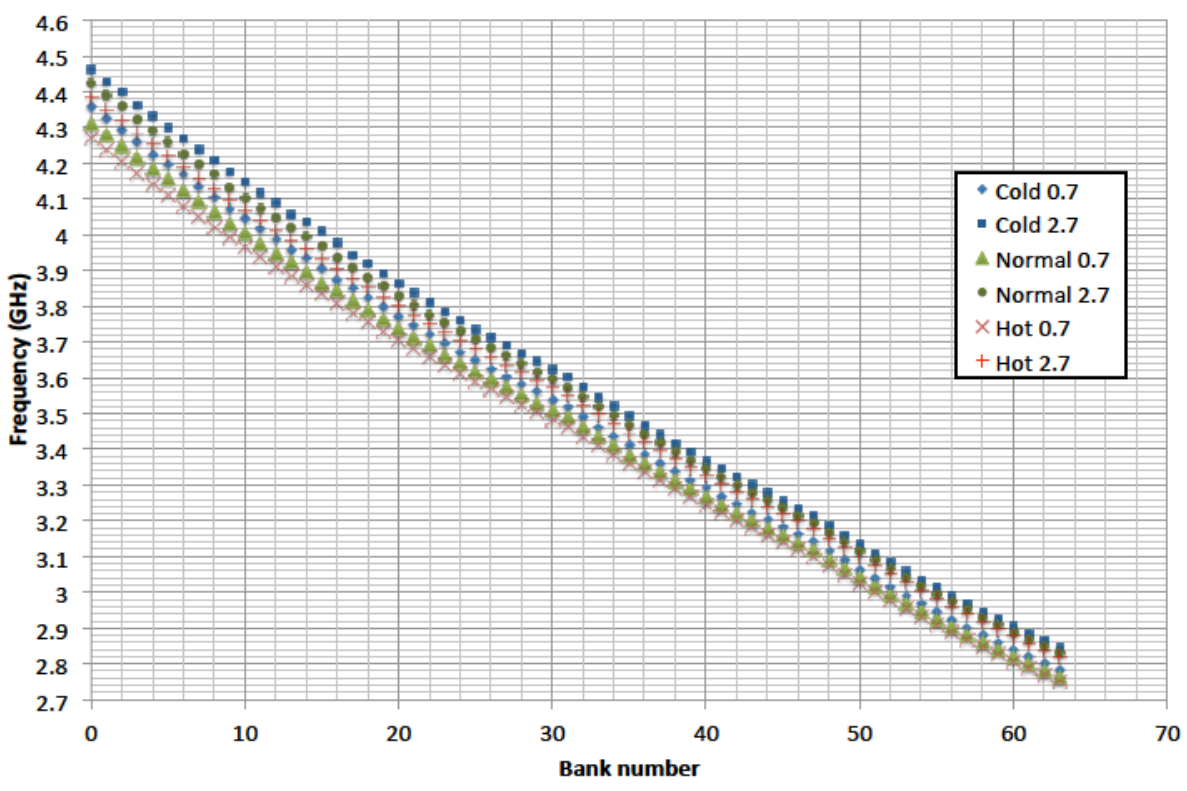

Figure 4.25: Plot of the simulated frequency vs frequency band code setting at high and low control voltages at hot, nominal and cold temperatures with the current setting GM1 


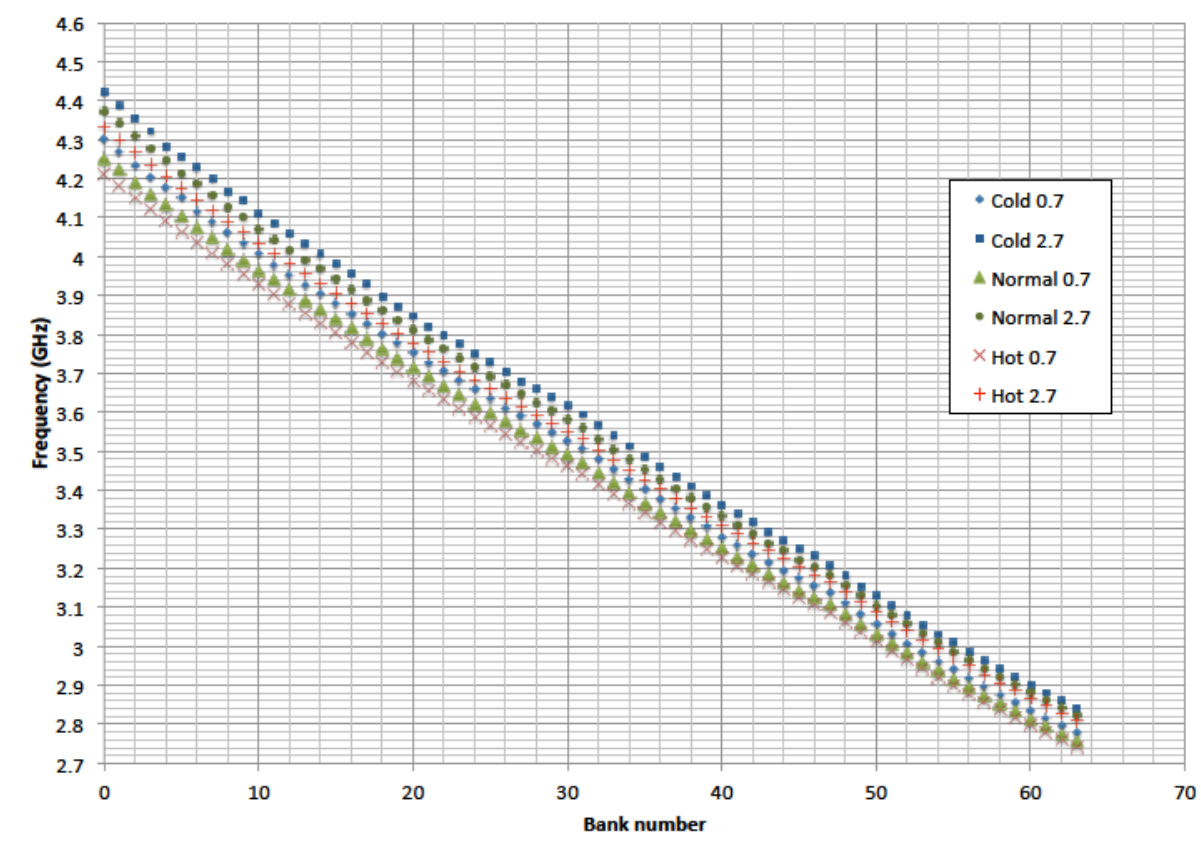

Figure 4.26: Plot of the simulated frequency vs frequency band code setting at high and low control voltages at hot, nominal and cold temperatures with the current setting GM2

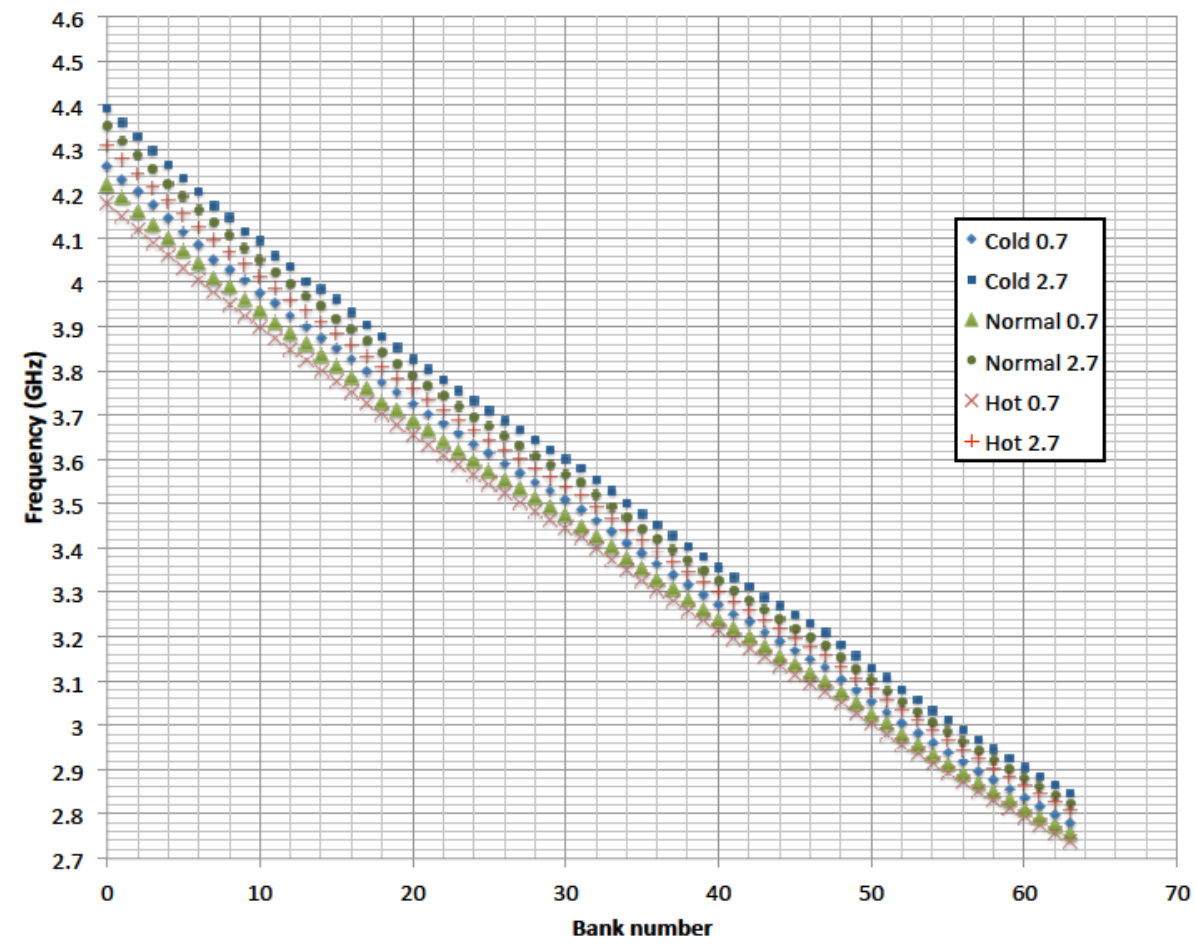

Figure 4.27: Plot of the simulated frequency vs frequency band code setting at high and low control voltages at hot, nominal and cold temperatures with the highest current setting GM3 


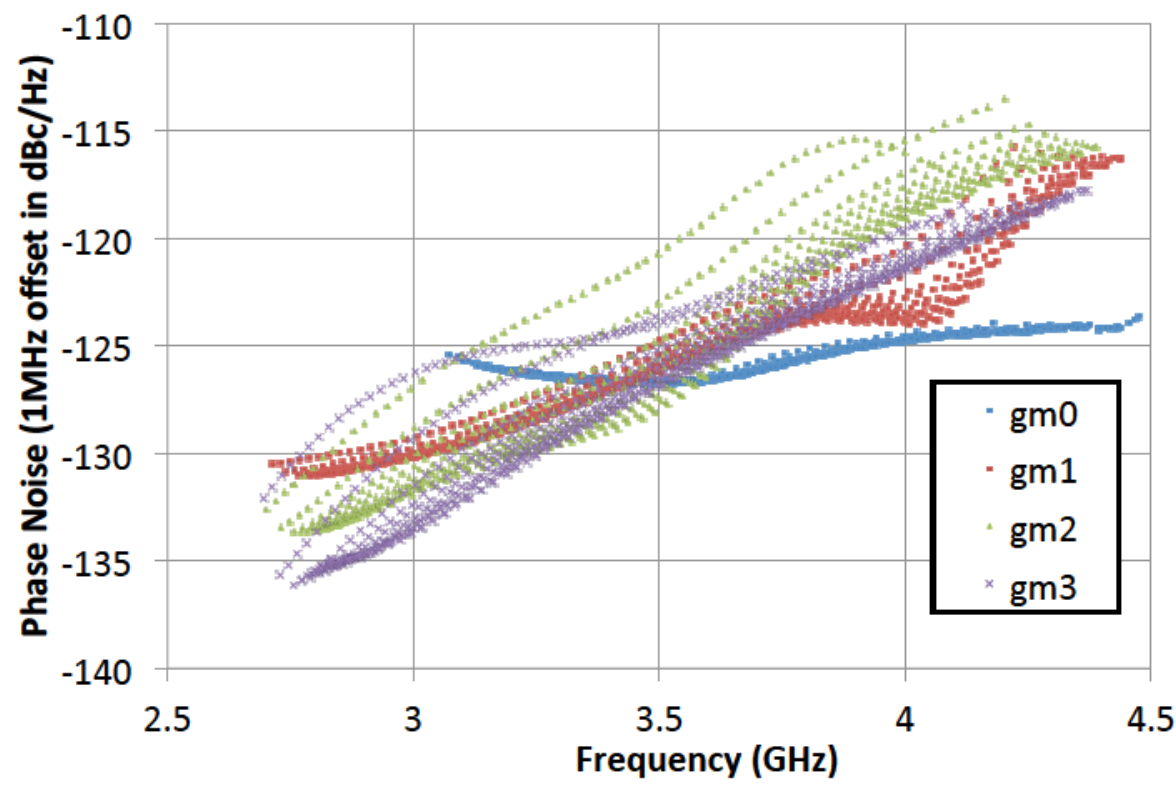

Figure 4.28: Plot of the simulated phase noise at a $1 \mathrm{MHz}$ offset vs frequency for different bank, control voltage and current settings

power efficiency figure of merit vs VCO frequency respectively. These are plotted against oscillation frequency, with results taken at multiple control voltages and bank settings, and are done at $25^{\circ} \mathrm{C}$ in a typical corner. The design of the maximum and minimum current settings were chosen to optimize for the lowest and highest frequencies respectively, with the two other settings spaced evenly between these two. This is evident by looking at the settings for optimal performance, which can be achieved almost completely using these two bank settings. However, the two middle settings still perform a useful role, since they allow for lower power consumption, and serve as a way to trade off performance to reduce power consumption. In addition, because of the lack of accurate parasitics, the additional bank settings become useful in being able to have more control over the oscillator performance to account for parasitics and process variation.

Fig. 4.30 shows a plot of phase noise vs offset frequency for a $3.2 \mathrm{GHz}$ band with the maximum current setting, at different process corners. Table 4.3 shows the phase noise at a $1 \mathrm{MHz}$ offset, along with the power consumption and the associated figure of merit. The use of the band gap generator helps stabilize the bias current, resulting in reasonably constant bias currents. 


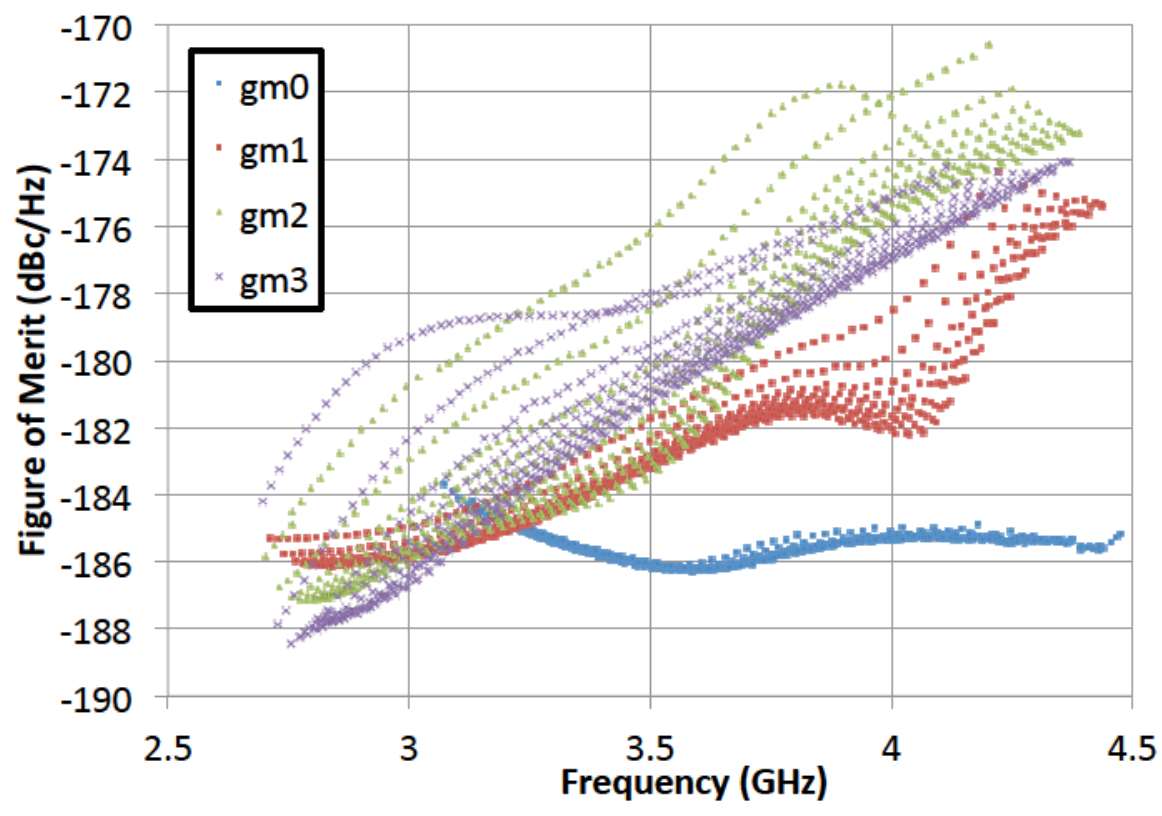

Figure 4.29: Plot of the simulated VCO figure of merit vs frequency for different bank, control voltage and current settings

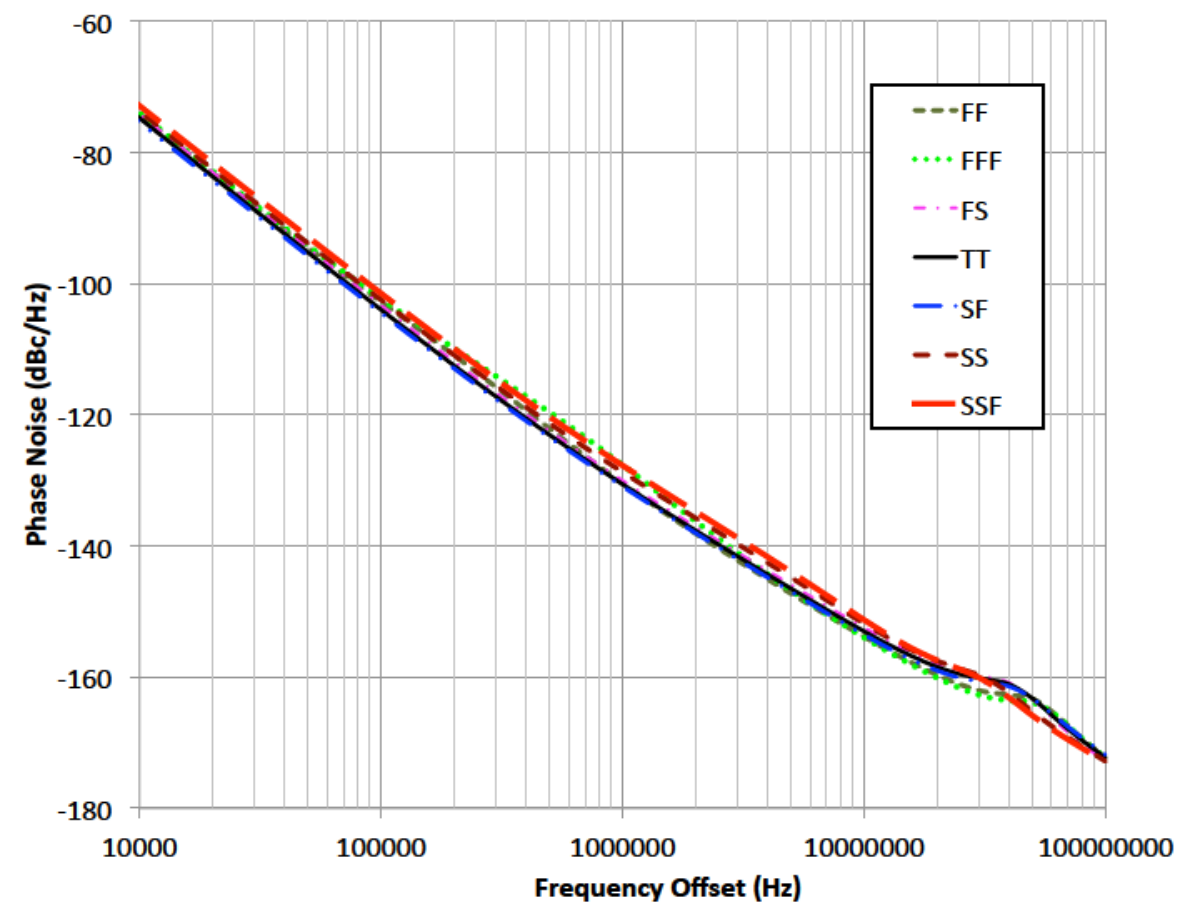

Figure 4.30: Plot of the simulated VCO vs offset frequency over different process corners 


\begin{tabular}{|c|c|c|c|c|}
\hline Corner & $\begin{array}{c}\text { Phase noise } \\
\text { at 1 MHz } \\
(\mathrm{dBc} / \mathrm{Hz})\end{array}$ & $\begin{array}{c}\text { DC Power } \\
(\mathrm{mW})\end{array}$ & Freq. (GHz) & $\begin{array}{c}\text { Figure of } \\
\text { Merit } \\
(\mathrm{dBc} / \mathrm{Hz})\end{array}$ \\
\hline $\mathrm{fff}$ & -127.6 & 46.88 & 3.242 & -181.2 \\
$\mathrm{ff}$ & -130.5 & 46.58 & 3.223 & -183.9 \\
$\mathrm{sf}$ & -130.9 & 46.23 & 3.171 & -184.3 \\
$\mathrm{fs}$ & -130.0 & 43.32 & 3.192 & -183.7 \\
$\mathrm{tt}$ & -130.6 & 44.72 & 3.186 & -184.1 \\
$\mathrm{sf}$ & -128.7 & 42.71 & 3.152 & -182.4 \\
$\mathrm{ssf}$ & -127.7 & 42.11 & 3.134 & -181.4 \\
\hline
\end{tabular}

Table 4.3: Simulated VCO performance at different process corners

\subsection{Overall Chip Layout}

As described earlier, the test chip was manufactured in the IBM 7WL 0.18 $\mu \mathrm{m}$ SiGe BiCMOS process. Fig. 4.31 shows a photograph of the test chip. The chip measures $2.7 \mathrm{~mm}$ by $2.6 \mathrm{~mm}$, giving a total area is $7.6 \mathrm{~mm}^{2}$. Much of the chip area is used for the on-chip loop filter (which is dominated by the loop capacitor) at $1.0 \mathrm{~mm}^{2}$, while the divider uses $0.5 \mathrm{~mm}^{2}$ and the digital interface and the $\Delta$ - $\Sigma$ modulator takes up $0.5 \mathrm{~mm}^{2}$. The VCO uses $0.7 \mathrm{~mm}^{2}$, the pads use $0.4 \mathrm{~mm}^{2}$, and voltage regulation and filtering takes up $0.1 \mathrm{~mm}^{2}$. Much of the remaining space (more than $2.5 \mathrm{~mm}^{2}$ ) is unused.

By looking at the amount of unused silicon area and by looking at the layout in Fig. 4.31, it is evident that the floor plan of the test chip is inefficient. Much of this is due to the fact that the test chip was based off of an existing design, which consisted of the lower half of the chip (loop filter, dividers, digital interface and modulator) with the VCO in the lower left corner. The new VCO was found to be too large to fit in the same area and was instead placed above the loop filter, extending the chip boundary. This was done rather than a slight rearrangement of the pads (as would be the normal solution) partially to limited time constraints, partially due to avoid changing the pad frame, partially due to the absence of critical verification tools which would be needed if modifications in the existing design were made, and partially due to the low cost of the test chip area. A minor draw back is the long VCO wires connecting the VCO output buffer to the divider. However, this was done on the low resistance top metal layers, and was found to have a low insertion loss.

In order to test the chip, it was packaged and wire-bonded into a QFN package, which was surface mounted onto a PCB. Fig. 4.32 shows a photograph of the PCB, showing some of the critical components, such as the test chip itself, the quartz crystal, power supply pins, output SMA connections and I2C interface bus. 


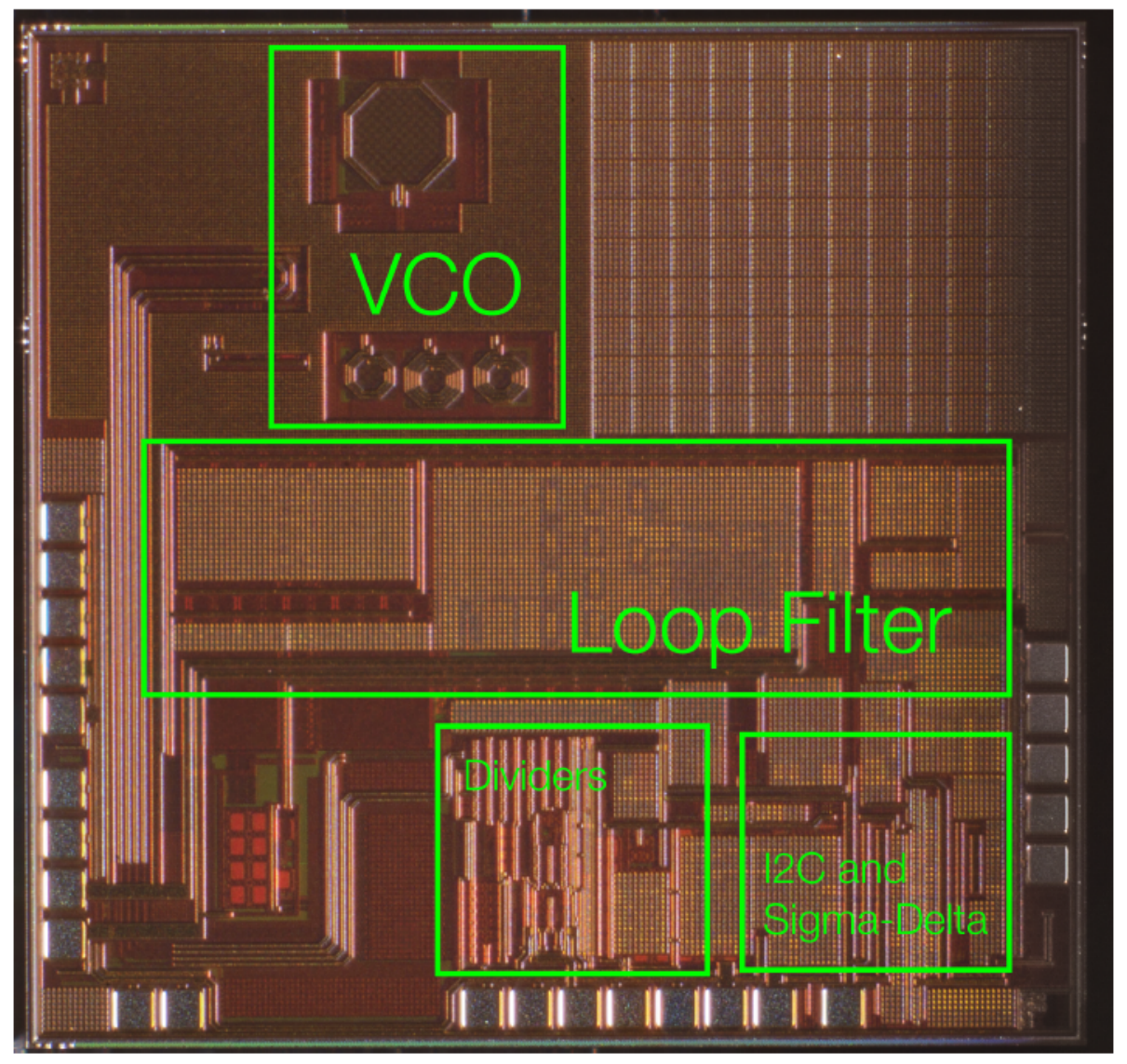

Figure 4.31: Photograph of the manufactured test chip 


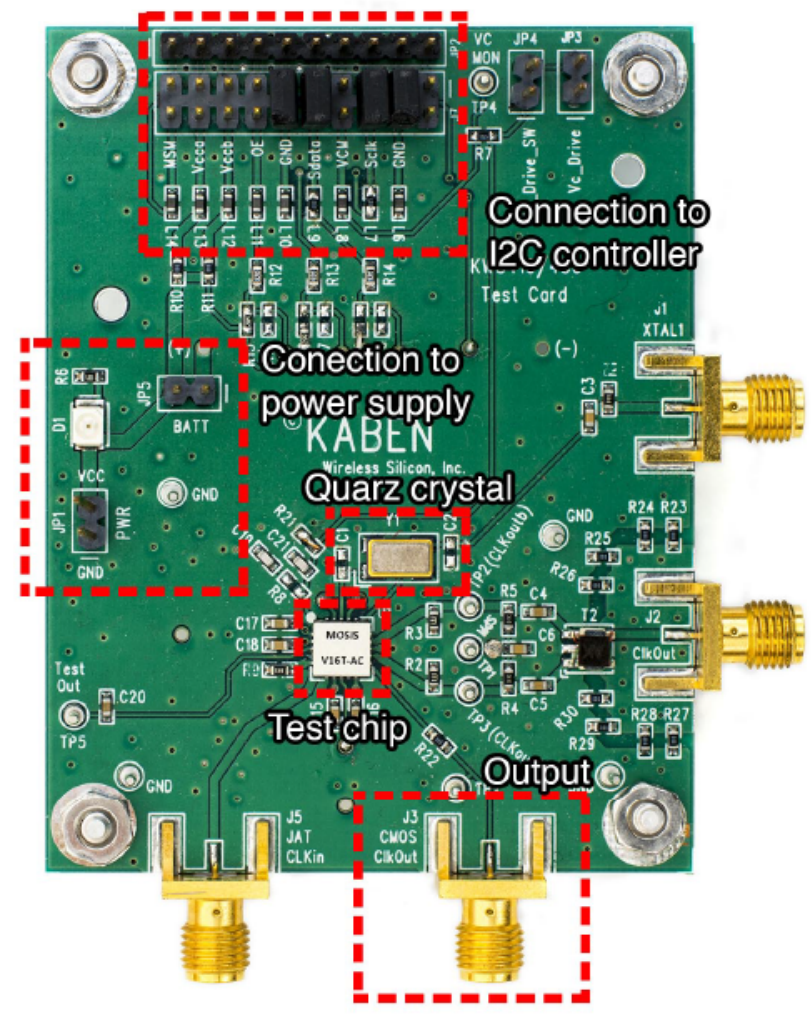

Figure 4.32: Photograph of a sample printed circuit board used to measure the test chip 


\subsection{Unresolved Issues}

As is typical with many designs, there were engineering tradeoffs that resulted in limitations to the content on the test chip as well as some seemingly non-optimal decisions. These were the result of several factors: limited design time dictated by foundry MPW tape-out dates), the lack of licenses for layout parasitic extraction tools, and the lack of licenses for the LVS tool used on the original PLL that was modified to develop the test chip. While the LVS tool used for the original PLL was not available, a license for a different LVS tool was used for the tape-out. However, a different layout and pin strategy was required in order for the new tool to properly recognize substrate ground connections. Thus, the only blocks that could be properly verified were blocks that were created from scratch, or heavily modified blocks from the original design. This resulted in several issues that were known and accepted at the time of tape-out. The original intention was a second version resolving these issues once the first test chip demonstrated the correct operation of the circuitry, but this was not possible due to various external circumstances. - the parasitics for the VCO could not be extracted due to a lack of extraction tool licenses. Therefore, the parasitics were estimated by visual inspection and correlation with previous test chips, and back-annotated onto the schematic in the design process. This leads to fairly inaccurate estimates for parasitics, and thus limits the ability of the simulations to match measurements. This is compensated through the use of a wide tuning range, and the multiple current settings. - the need for to create blocks either from scratch or from heavily modified original versions limited the number of modifications that could be made to existing circuitry. This resulted in the inefficient use of chip area (where the VCO was placed in the far corner rather than rearranging the blocks to minimize area), and limited the number of control pins available. This resulted in long output lines, and long power and ground traces to the VCO. More critically, the loop filter was referenced from the power supply rather than the $\mathrm{VCO}$, since the necessary connection to make this possible were found to be too difficult to change and verify in the time required. This, unfortunately led to larger power supply noise coupling than originally intended, but this would be partially remedied by the use of the lowest noise power supplies available during the measurement phase. - lack of time also prevented the creation of any break-out circuitry, such as to measure the tank inductor or the tank resonator on its own, or the VCO on its own. 


\section{Chapter 5}

\section{Measurement results}

As discussed in the previous chapter, a test chip was designed to demonstrate the concept of using a sub-threshold switching circuit with VCO capacitor banks to implement gradual frequency band shifts to allow a PLL to change frequency bands while reducing the transient

effects and preventing cycle slipping. The following chapter details the measurement results of this test chip.

\section{$5.1 \quad$ Test Setup}

Most of the measurements of the PLL were made using the test setup shown in Fig. 5.1. Here the PLL board (acting as the device under test or DUT) is connected to an Agilent E3646A power supply and controlled by a National Instruments I2C controller connected to a PC, which is running the user interface. The output of the PLL is fed into a National Instruments 5663 digitizer in a PXIe-1075 chassis. The internal structure of the digitizer is shown: it consists of a down conversion mixer feeding into a $150 \mathrm{MS} / \mathrm{s}$ 16-bit ADC. The input bandwidth and the oscillator frequency is controlled by software on the chassis, which allows it to perform a variety of measurements and emulate many types of measurement equipment. Most of the PLL measurements made were done using the chassis configured as a spectrum analyzer. This was preferable to using a hardware spectrum analyzer due to ease of use, reduced measurement time and a lower noise floor. Some of the measurement results were duplicated using an HP 8564E spectrum analyzer to validate the use of the NI-5663. 


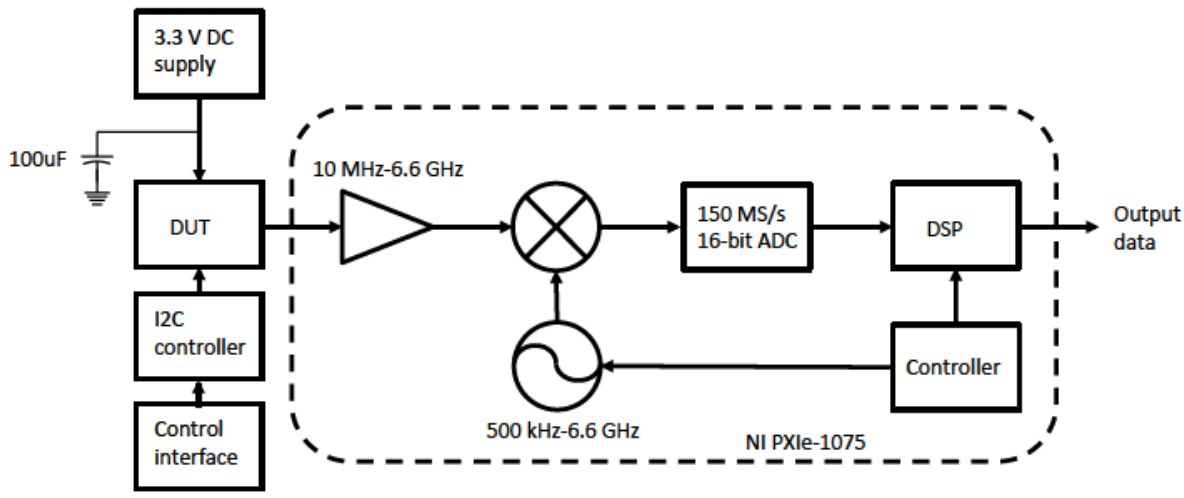

Figure 5.1: Diagram showing the test setup for most of the measurements of the PLL

\subsection{General PLL Operation}

As was previously discussed, the proposed circuit should not adversely affect the phase noise or jitter performance of the PLL when it is operating in locked operation: the circuit should only impact performance when switching frequency bands. Thus, measurements were made on the PLL in locked operation, since good jitter performance would demonstrate the absence of any adverse effects from the slow switching circuit. The following is a summary of the measurements taken from the PLL while in locked operation.

The phase noise of the PLL was measured using a National Instruments NI 5663 digitizer configured to operate as a spectrum analyzer. The various VCO and charge pump current settings were exercised in order to determine the ideal current settings to minimize the PLL jitter and phase noise across different frequency ranges. Fig. 5.2 shows a plot of the integrated jitter from $10 \mathrm{kHz}$ to $10 \mathrm{MHz}$ for the lowest two output division ratios with different current settings. As can be seen, the 3 highest settings each have different regions where they provide the lowest jitter, thus allowing the combination of all 3 to have low jitter across a wide frequency range. The lowest VCO current setting was not found to have optimal performance, and was found to have a limited frequency range. This is likely due to underestimated parasitics, which will be discussed further later in this section.

Plot 5.3 shows a sample phase noise plot of the PLL at $1.05 \mathrm{GHz}$, where the integrated RMS jitter from $10 \mathrm{kHz}$ to $10 \mathrm{MHz}$ is $0.365 \mathrm{ps}$. The DC power consumption at this frequency is $81 \mathrm{~mW}$. Similar measurements were taken from the entire PLL's operating range. A plot showing the integrated RMS jitter (both in terms of absolute time and relative to the frequency in degrees) is shown in Fig. 5.4.

A plot of the phase noise at different PLL frequencies can be seen in Fig. 5.5. The use of the incorrect loop filter reference can be seen in the shared ripple patterns in the phase 


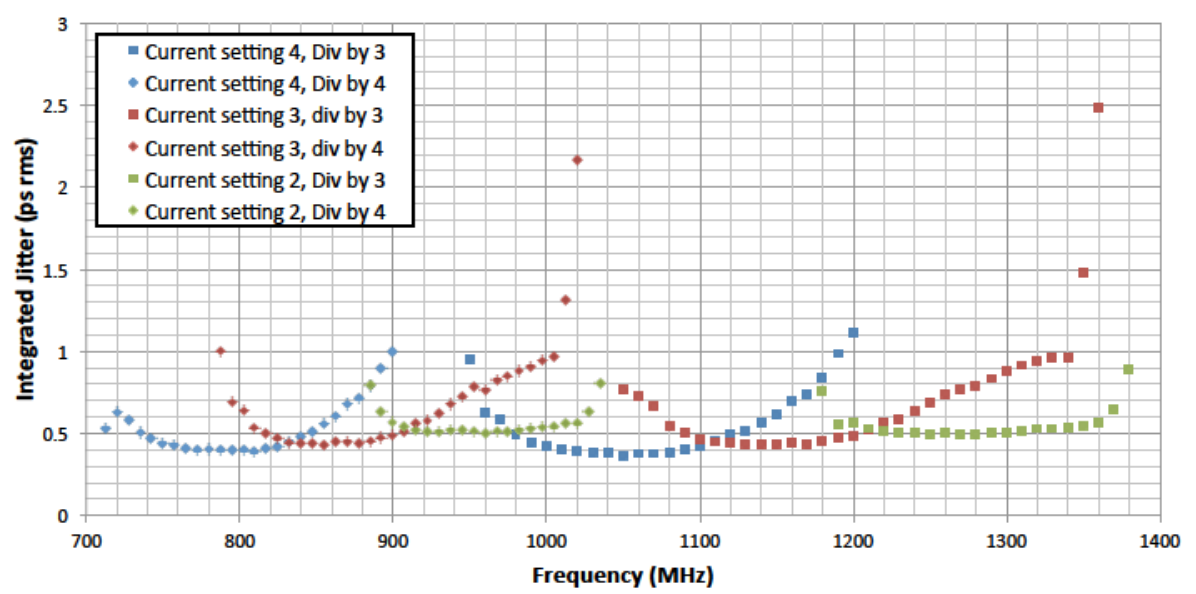

Figure 5.2: Plot of the integrated jitter vs. frequency for different VCO frequencies

noise, despite the use of the lowest noise DC supply available. The VCO phase noise can be examined from these plots by looking at the phase noise well outside of the loop bandwidth. When taking the output division ratio into account, the phase noise at a $1 \mathrm{MHz}$ offset is significantly higher than what would normally be expected. In addition, the frequency range for which the VCO could oscillate for each current setting was found to be smaller than predicted. The most likely explanation is this is resistive losses in the VCO, particularly in the wiring between the gain transistors, the inductor and the capacitor bank. These losses were likely underestimated in the design process resulting in poorer performance, and will be addressed later in this section. Other factors were considered and dismissed, such as the jitter added by the programmable divider or the buffer chain (which would contribute more to the in-band noise), process variation (corner simulations showed the effect of corner variations on VCO performance is minimal), and incorrect bias point generation (measurements of the supply current showed that the bias currents were as designed, and there was no evidence to suggest that the voltage regulators produced an incorrect output voltage).

The output spectrum of the PLL (again set at $1.05 \mathrm{GHz}$ ) was measured with National Instruments NI 5663 digitizer configured to operate as a spectrum analyzer. The resolution bandwidth was set to $10 \mathrm{kHz}$ and over 1000 samples of data captured over a period of several minutes were averaged (effecting a very low video bandwidth). The plot of the measurement results can be seen in Fig 5.6. This plot shows the maximum spur in this frequency range of $-66.1 \mathrm{dBc}$. The spur pattern is asymmetrical and irregular due to the non-linear loop filter capacitance, which is caused by the poly-silicon passives making up the on-chip loop filter.

The in-band spurs when operating with a fractional-N PLL can be measured by looking at the phase noise. This is shown in Fig. 5.7, This was done with the PLL frequency set to 


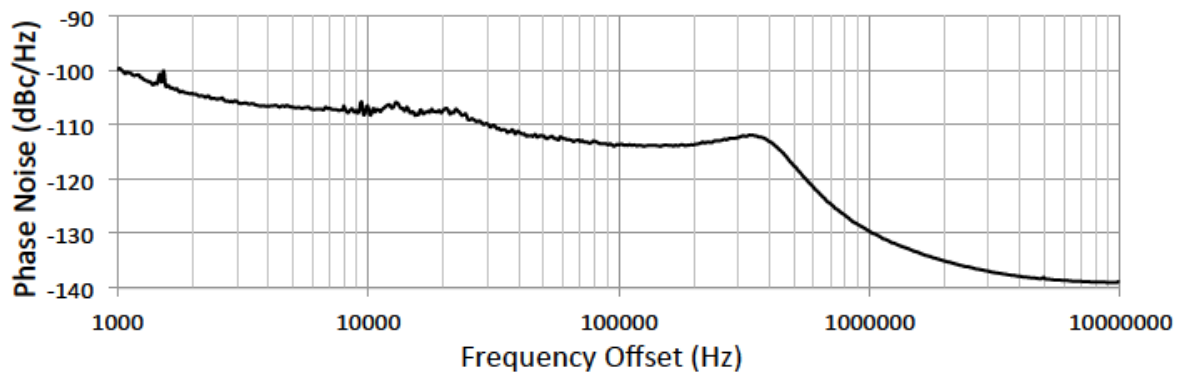

Figure 5.3: Plot of of the phase noise of the PLL in integer-N mode with an output frequency at $1.05 \mathrm{GHz}$.

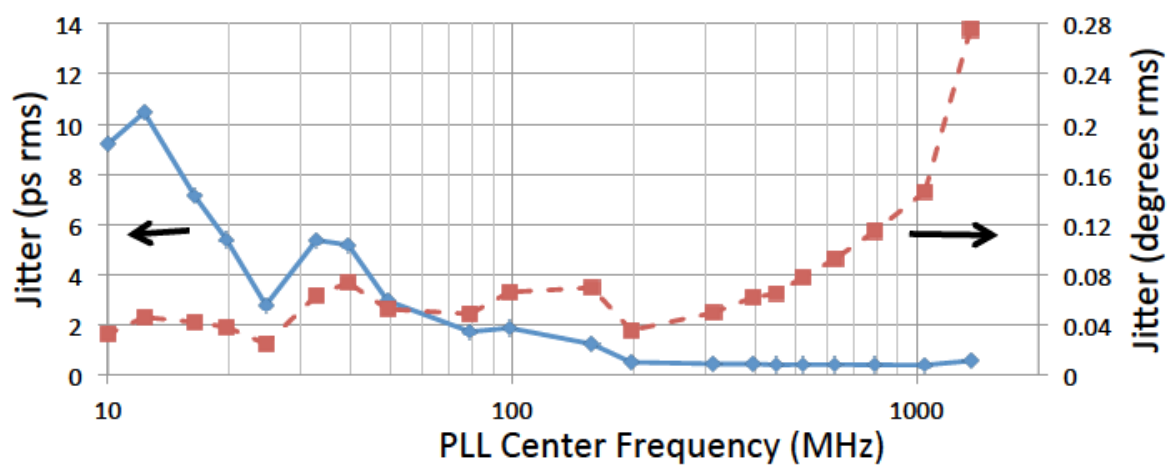

Figure 5.4: Plot of the integrated jitter of the PLL over its operating range 


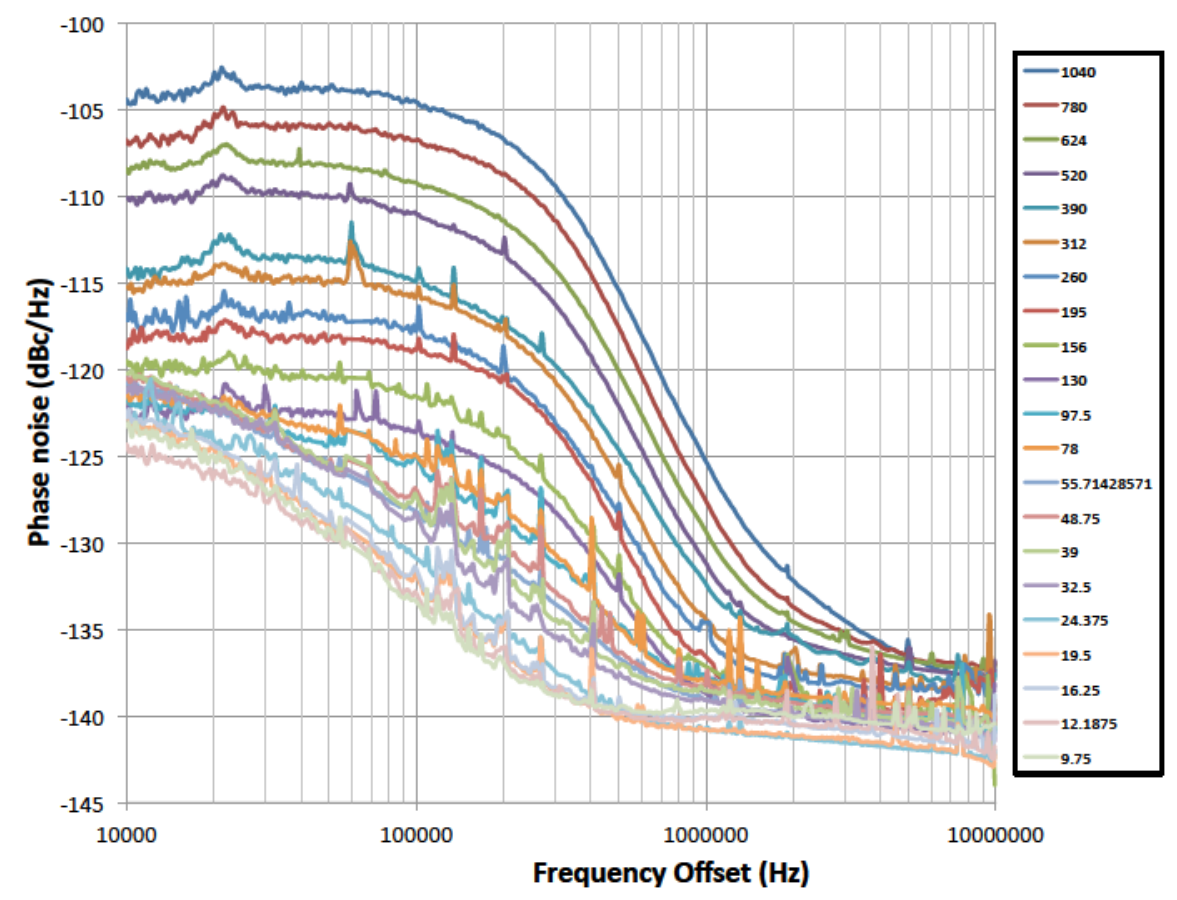

Figure 5.5: Plot of the PLL phase noise across its frequency range

$1.0500005 \mathrm{GHz}$. The in-band spurs for this frequency is $-53 \mathrm{dBc}$.

The performance of the PLL can be compared to other published measured results of PLLs. In Table 5.1, the jitter, and power consumption is compared to other designs with a similar frequency range. In order to compare designs with different tradeoffs between DC power consumption and noise, the figure of merit proposed in [192] is used, as shown in equation (5.1):

$$
F o M=10 \log _{10}\left(\left(\frac{\sigma}{1 s}\right)^{2} \frac{P_{D C}}{1 \mathrm{~mW}}\right)
$$

where $\sigma$ is the integrated jitter of the PLL and $P_{D C}$ is the DC power consumption of the PLL. As is evident through a comparison of the Figures of Merit, the PLL shown here has comparable performance to that of published results, demonstrating that the modifications shown here are compatible with a high performance PLL.

While Table 5.1 shows that the PLL showed performance comparable to other published frequency synthesizers, it is evident that many of the design compromises made resulted in performance degradations that could have been improved. This includes the incorrect power supply connection of the PLL loop filter (which was done due to limitations in verification tool licenses) which resulted in more power supply noise coupling. It also includes the increased VCO phase noise and reduced oscillation range which is likely due to underestimated parasitic 


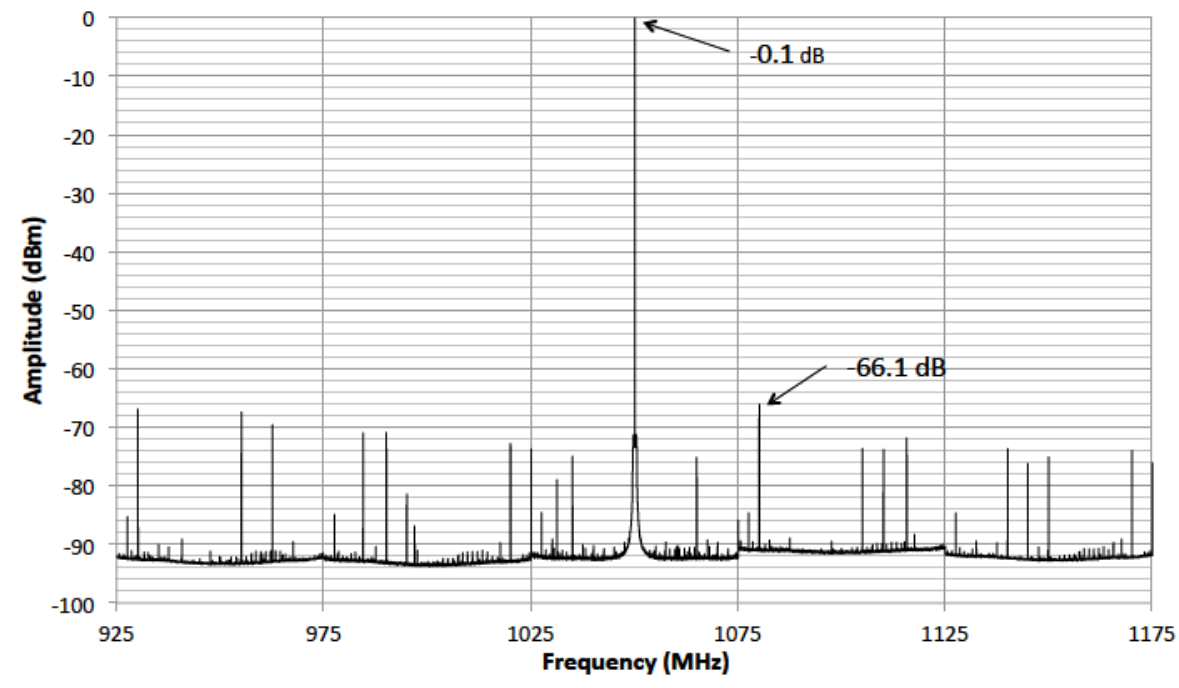

Figure 5.6: Spectrum plot showing PLL out-of-band spurs in integer-N mode with an output frequency at $1.05 \mathrm{GHz}$.

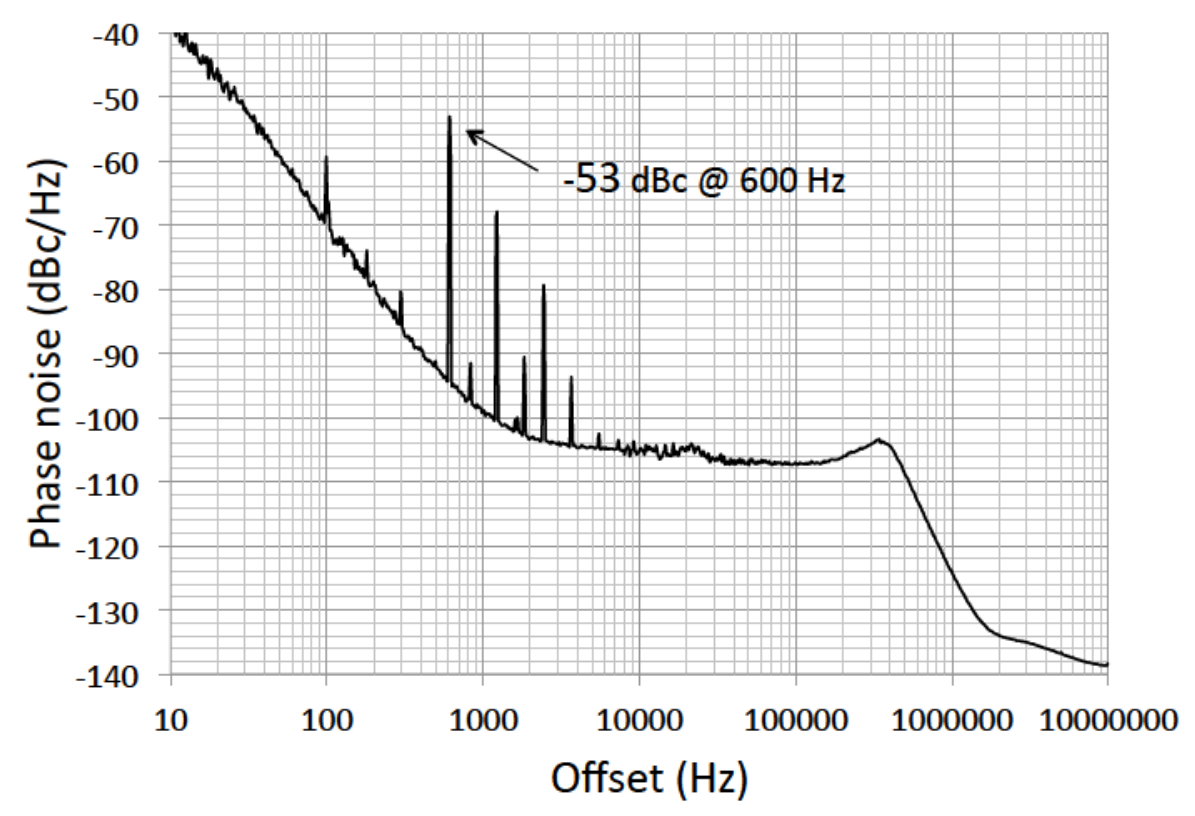

Figure 5.7: Spectrum plot showing PLL in-band spurs in fractional-N mode with an output frequency at $1.0500005 \mathrm{GHz}$. 


\begin{tabular}{|c|c|c|c|c|c|c|c|c|c|c|}
\hline & $\begin{array}{l}\text { This } \\
\text { Work }\end{array}$ & [193] & [194] & [195] & [196] & [197] & [198] & [199] & [171] & [172] \\
\hline Freq. (MHz) & $8-1400$ & $100-4267$ & $54-862$ & $100-5000$ & $55-960$ & $50-4800$ & $30-650$ & $40-1075$ & $480-1000$ & $74-1973$ \\
\hline Process & $\begin{array}{l}0.18 \mu \mathrm{m} \\
\text { BiCMOS }\end{array}$ & $\begin{array}{l}65 \mathrm{~nm} \\
\text { CMOS }\end{array}$ & $\begin{array}{c}0.18 \mu \mathrm{m} \\
\text { CMOS }\end{array}$ & $\begin{array}{l}45 \mathrm{~nm} \\
\text { CMOS }\end{array}$ & $\begin{array}{c}0.18 \mu \mathrm{m} \\
\text { CMOS }\end{array}$ & $\begin{array}{c}0.18 \mu \mathrm{m} \\
\text { CMOS }\end{array}$ & $\begin{array}{l}0.13 \mu \mathrm{m} \\
\text { CMOS }\end{array}$ & $\begin{array}{l}0.13 \mu \mathrm{m} \\
\text { CMOS }\end{array}$ & $\begin{array}{c}0.13 \mu \mathrm{m} \\
\text { CMOS }\end{array}$ & $\begin{array}{c}0.13 \mu \mathrm{m} \\
\text { CMOS }\end{array}$ \\
\hline $\begin{array}{l}\text { In-band noise } \\
\text { floor } \\
(\mathrm{dBc} / \mathrm{Hz})\end{array}$ & $\begin{array}{c}-108 \text { to } \\
-114\end{array}$ & N/A & $\begin{array}{l}-90 \text { to } \\
-100\end{array}$ & $-82-86$ & $\begin{array}{c}-90 \text { to } \\
-90\end{array}$ & -90 & $\mathrm{~N} / \mathrm{A}$ & -88 & -110 & -86.5 \\
\hline $\begin{array}{l}\text { Phase noise } \\
(\mathrm{dBc} / \mathrm{Hz})\end{array}$ & -130 & -110 & -122 & -122 & -123 & -100 & N/A & -125 & -119 & -124 \\
\hline $\begin{array}{l}\text { Phase noise } \\
\text { offset freq. }\end{array}$ & $1 \mathrm{MHz}$ & $1 \mathrm{MHz}$ & $1 \mathrm{MHz}$ & $2 \mathrm{MHz}$ & $1 \mathrm{MHz}$ & $100 \mathrm{kHz}$ & $\mathrm{N} / \mathrm{A}$ & $1 \mathrm{MHz}$ & $1 \mathrm{MHz}$ & $1 \mathrm{MHz}$ \\
\hline $\begin{array}{l}\text { Phase noise } \\
\text { freq. (MHz) }\end{array}$ & 1050 & 1280 & 862 & 7200 & 960 & 4800 & $\mathrm{~N} / \mathrm{A}$ & 900 & 600 & 1492 \\
\hline $\begin{array}{l}\text { DC Power } \\
(\mathrm{mW})\end{array}$ & $54-81$ & $3.6-8.4$ & $40-83$ & $22-32$ & 40 & 94 & 7 & 16.8 & 22 & 18.96 \\
\hline $\begin{array}{l}\text { RMS Jitter } \\
\text { (ps) }\end{array}$ & 0.365 & 1.72 & 1.61 & 0.734 & 4.80 & 0.380 & 4.2 & 2.19 & 0.94 & 1.20 \\
\hline $\begin{array}{l}\text { Figure of } \\
\text { Merit (dB) }\end{array}$ & -230 & -228 & -217 & -229 & -210 & -229 & -219 & -221 & -227 & -226 \\
\hline
\end{tabular}

Table 5.1: Comparison of performance to other published works

resistance between the gain transistors and the oscillator resonator. Because of the lack of extraction tool licenses, parasitics could only be estimated by relying on the process kit model accuracy, and back annotation based on hand calculations or extraction results from previous designs. The design relied heavily on the accuracy of the RF FET models used in the process, although the very low simulated noise figure numbers given in the transistor characterization process shows that wiring and gate resistances are either underestimated or omitted. In addition, wiring parasitics from the cross-coupled pairs to the capacitor bank or to the inductor were likely also underestimated.

\subsection{Temperature Measurements}

Section 4.10.7 demonstrated through simulations that the frequency range of each frequency band shifts with temperature. Measurements of the frequency synthesizer using an temperature controlled environmental chamber were used to verify this. Measurements done by varying the supply voltage also showed that changes in the supply voltage also affect the range of the frequency synthesizer, as lower supply voltages limit the maximum VCO control voltage that can be applied. Since in both cases, the frequency band is limited by the range of possible VCO control voltages, a feedback circuit can be implemented, such as that shown in Fig. 5.8, which is based on control voltage monitoring [161]. If it starts to approach either extremity, a frequency band switch is called. A minimum time between 


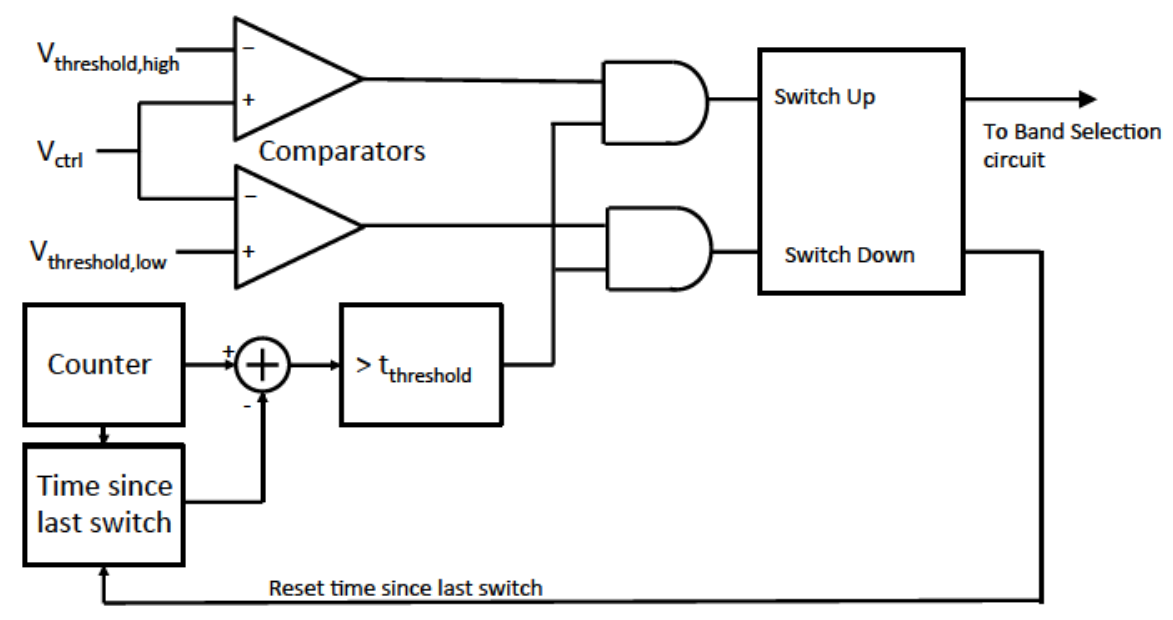

Figure 5.8: Proposed feedback circuit for band switching control based on control voltage monitoring

band switches can be enforced to prevent oscillation between two frequency bands, and the low frequency nature of stimulus that would require these band switches (temperature drifts, supply voltage sags such as from a dying battery, etc.) also help prevent oscillation. This is supported by measurements of the frequency synthesizer, which showed an approximate thermal time constant of several minutes.

Temperature measurements were conducted to simulate the effect of this circuit through manual control. This was done by placing the test board inside an environmental chamber. The frequency synthesizer was set to $1089 \mathrm{MHz}$ and the temperature was changed from -25 $\mathrm{C}$ to $25 \mathrm{C}$, once with no band switching and once using a band switching algorithm based on control voltage monitoring by the frequency band is switched when the control voltage approaches one of its extremes. As Fig. 5.9 shows, without band switching, the circuit loses lock when the control voltage reaches the maximum value of $3.3 \mathrm{~V}$. With band switching, the band switches when the control voltage reaches $3.0 \mathrm{~V}$, allowing it to stay locked.

Similar measurements were done varying the supply voltage instead of the temperature. As shown in Fig. 5.10, the frequency synthesizer was set to $1084 \mathrm{MHz}$ initially, and the supply voltage was gradually lowered. If no band switching is used, the frequency synthesizer eventually loses lock. However, with the control voltage monitoring circuit, a band switch can be called when the control voltage gets too close to the supply voltage, allowing the system to maintain lock.

This demonstrates the validity of the simulation results indicating frequency shift due to temperature changes or supply voltage changes. Without the ability to change frequency bands, the PLL would not be able to maintain lock if the locking frequency fell outside of 

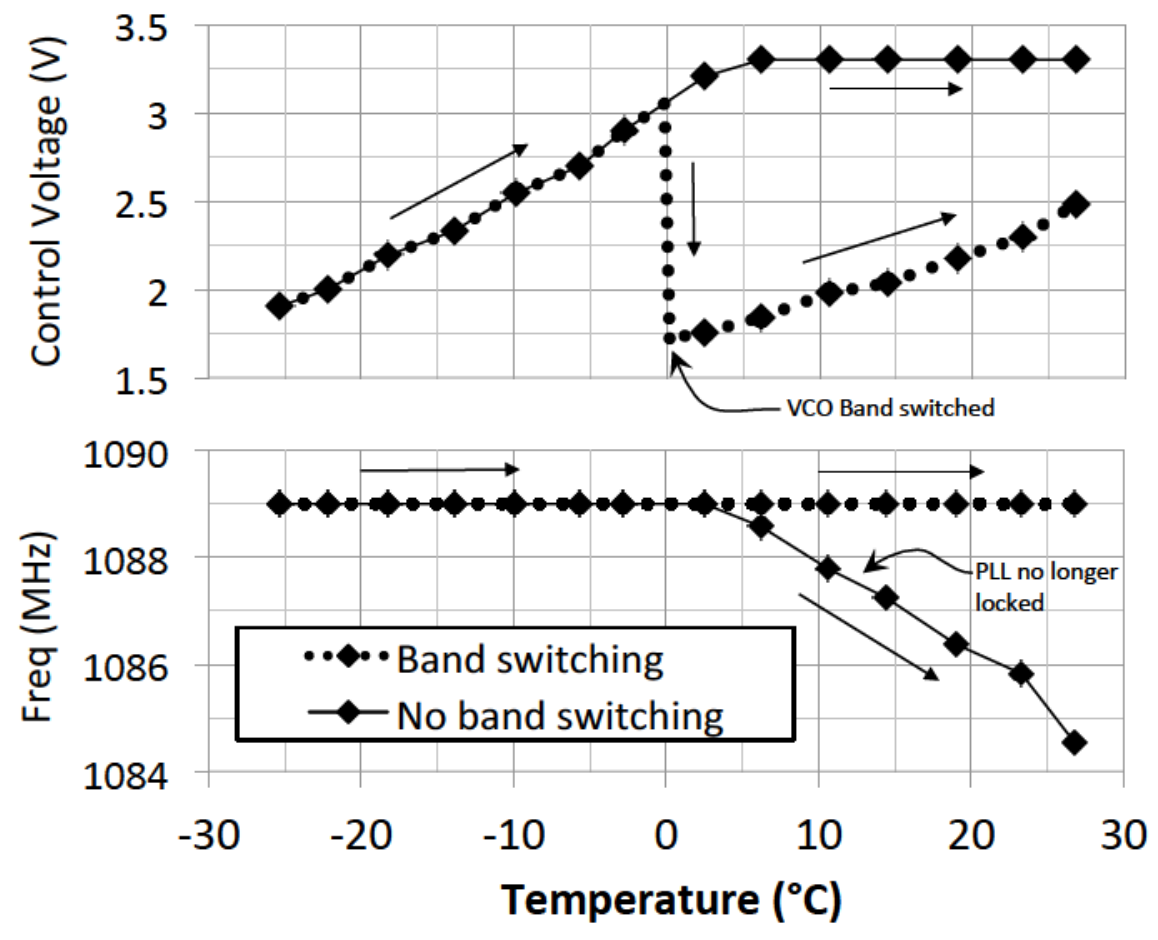

Figure 5.9: VCO Control voltage and output frequency of the frequency synthesizer with changing temperature. 


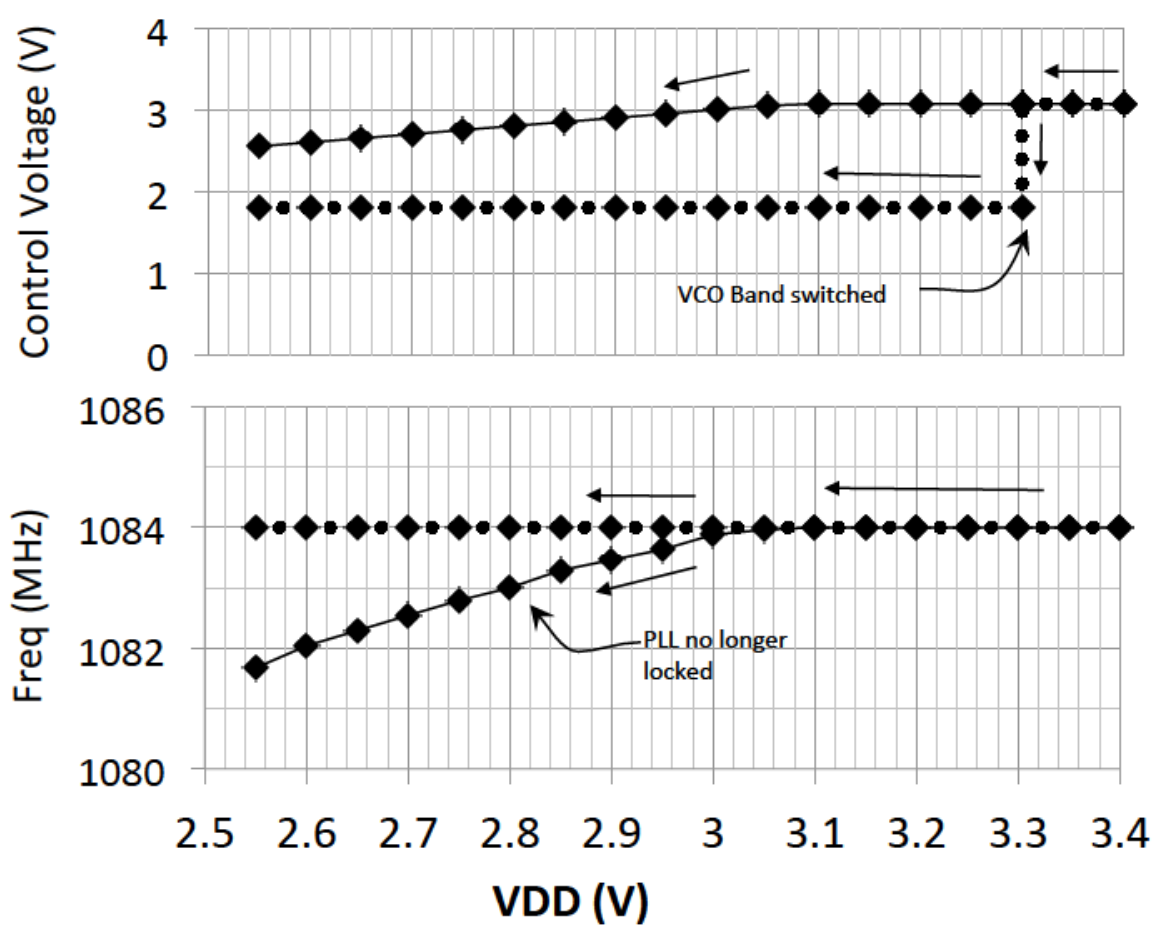

Figure 5.10: VCO Control voltage and output frequency of the frequency synthesizer with changing supply voltage.

the frequency band range. While in the case of a conventional frequency band switch this band switch would result in cycle slipping and a large frequency error, the proposed circuit would allow the frequency band to be changed while preventing cycle slipping, and limiting frequency and phase errors to a predetermined amount controllable by design parameters.

\subsection{Frequency Band switching circuit}

Testing the frequency band switching circuit required a more complex setup due to the nature of the measurements required. High accuracy frequency measurements require long averaging times with resolution bandwidth in the $\mathrm{kHz}$ region or lower. Similarly, oscilloscopes rely on periodic signals over a very short period of time. In order to properly characterize the frequency band switching circuit, accurate instantaneous frequency vs. time measurements are required over a long period of time. In order to perform this set of measurements, the National Instruments NI 5663 digitizer was configured as a sampling oscilloscope by directly sampling the data at the maximum sample rate of $150 \mathrm{MS} / \mathrm{s}$. This was done over a period of 2 seconds, which is long enough to ensure that the externally activated frequency switching transient is captured. The instantaneous frequency can be calculated from this set 
of transient data. To ensure that the waveform is sampled below the Nyquist frequency of $75 \mathrm{MHz}$, the PLL output is set to $56 \mathrm{MHz}$.

This method comes with issues of its own. Although the sample rate is not high relative to the frequency range of the PLL, this amount of sampled data results in 300 million data points, resulting in data files that are unwieldy to process. Extracting the frequency vs. time data from the transient sampled data is also not trivial. A common approach is to use a Discrete Fourier Transform (DFT), although this ends up having similar problems to the use of a spectrum analyzer, since the frequency resolution of a DFT (relative to the sampling frequency) is proportional to the number of transient data points used in the DFT in the first place. To obtain high resolution frequency data, the number of data points needed would be longer than the transient effects that are being measured, preventing resolution of the switching transient.

Instead, an interpolation approach is used which attempts to recover the zero crossings of the waveform to recover the length of the period of each cycle in the output waveform. This approach has many issues as well. Although the waveform is sampled above the minimum Nyquist rate needed, this still only gives on average 2.68 data points per period, which is insufficient data to recover the zero crossings using a low-order interpolation (such as linear or quadratic algorithms). However, since the output was found to be highly sinusoidal, based on visual inspection and analysis of the output harmonics, the waveform can be approximated by a high-order polynomial. This relies on the fact that the sine function can be written as a infinite polynomial series:

$$
\sin x=\sum_{n=0}^{\infty} \frac{(-1)^{n}}{(2 n+1) !} x^{2 n+1}
$$

Due to the rapidly increasing nature of the factorial term, higher-order polynomials contribute very little to the expression, thus allowing for a reasonable approximation of the sine function, provided the polynomial order is sufficiently high. Thus, to calculate the locations of the zero crossings, 80th order Lagrange polynomials are constructed near each zero crossings, and the actual crossing location is estimated using a first-order discrete implementation of Newton's method. The instantaneous frequency is calculated by taking the inverse of the difference between consecutive crossings. This approach results in numerical noise and artifacts in the calculated frequency values due to the fact that this method essentially attempts to vastly increase the sampling rate of the data and amplifies any noise, inaccuracy or artifacts. As a result, further processing in the form of low pass filtering is done, essentially lowering the noise by using multiple transient data points spread out over a longer period of time to calculate the frequency data. This results in improved accuracy and precision, while 


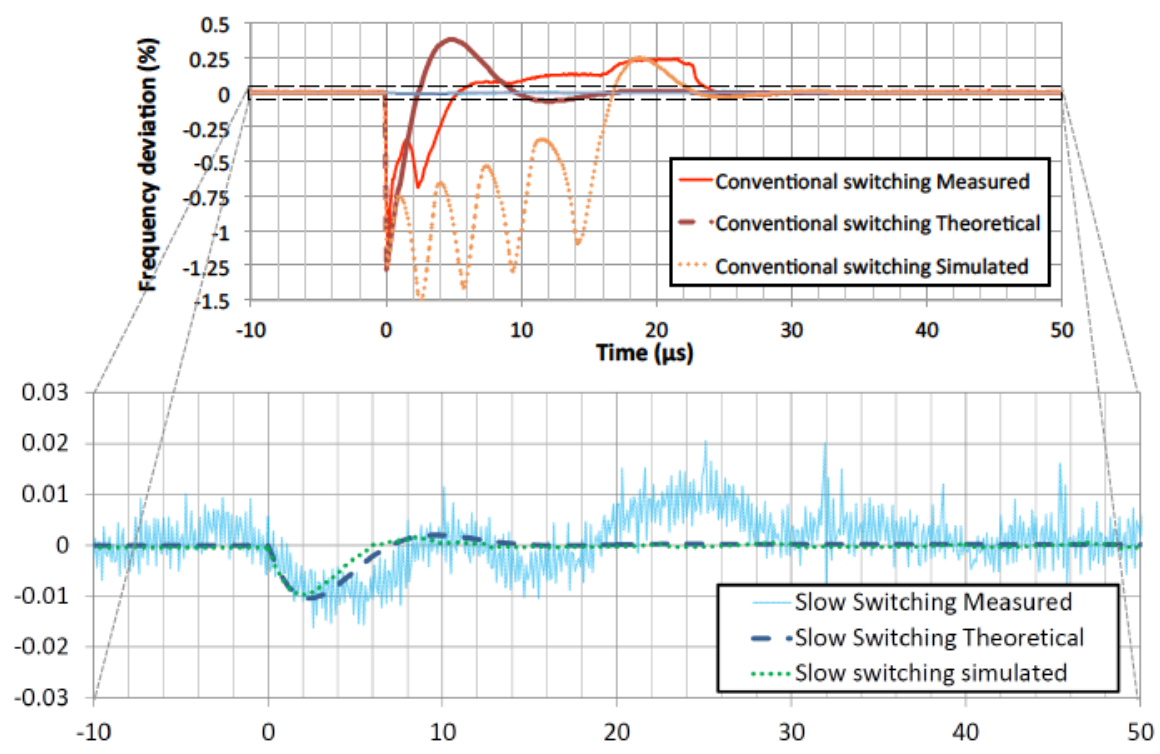

Figure 5.11: Plot of frequency deviation vs. time of the synthesizer (which is set to $58 \mathrm{MHz}$ ) while undergoing a VCO bank switch using regular switching and slow switching. Measured, simulated and theoretical results are shown.

requiring fewer data points than would be required using hardware frequency measurements or by calculating the DFT. Since the results gained by using this approach were found to be sufficient, neither further optimization of this algorithm nor research for possible improved algorithms were performed.

A plot of the final calculated results can be seen in Fig. 5.11. This plot shows the process measured frequency vs. time data during a band switch both with and without the band switching circuit compared to the expected results based on theoretical calculations and system simulations as discussed in Section 3.1.2. The difference between the simulated and theoretical results comes from the fact that the simulations are able to predict the presence of non-linear cycle slipping, which cannot be predicted by the linear theoretical calculations. The simulations also use a simple charge pump model, rather than the actual charge pump and phase detector used in the test chip. As is evident, without the proposed band switching circuit, the conventional frequency band switch results in cycle slipping, resulting in a large frequency deviation of $1.25 \%$. With the slow switching circuit, the maximum frequency error is reduced by $99.3 \%$ to a $0.009 \%$ error, while also preventing cycle slipping. This also reduces the phase error. While this cannot be directly measured, the maximum phase error is estimated to be less than 6 degrees from simulation results, while with cycle slipping present, more than 700 degrees of error are seen.

Another way of observing the behaviour of the circuit is to examine it in the frequency 
domain. This is done by measuring the output using a spectrum analyzer and looking for any effects caused by the switching circuit. In theory, the low phase error and the prevention of cycle slipping should greatly reduce any observable artifacts. However, there is still some difficulty in measuring this, since the spectrum plot is generated by sweeping the frequency of a narrowband band-pass filter. While this is suitable for measuring periodic phenomenon, it does not work well for measuring transient signals wth wide band spectral components that occurs only for a brief period of time. To work around this, a low video bandwidth is set by taking the average of many passes through the frequency range, while the frequency band is switched repeatedly at random intervals to ensure that a switching transient is captured. A comparison of the spectrum can be done both with the conventional frequency band switching and with the slow switching circuit.

A limitation of this measurement is the fact that the random, repeated band switches does not correlate well with how the circuit is intended to be used. Another limitation with this plot is the fact that the control signal controlling the band switch itself induces some noise into the circuitry, since the $\mathrm{I} 2 \mathrm{C}$ receiver on the chip is not perfectly isolated from the rest of the circuit. Thus, when settings are written onto the chip, significant noise is generated by the logic, which can couple to the VCO control voltage. The glitches caused by this circuit were found to be significant, being similar in size to the frequency offsets caused by band switching with the slow switching circuit active. While this can be separated from the transient data (since the glitch occurs before the band switch), it cannot be separated when performing frequency domain measurements over long periods of time. Thus, this adds a limitation on the accuracy of this plot.

The plot of the spectrum (measured with a resolution bandwidth of $10 \mathrm{kHz}$ with the NI 5663 configured as a spectrum analyzer), taken over a 1 minute period with random frequency band switches, can be seen in Fig. 5.12. In addition to the conventional and slow switching methods, a spectrum of the locked oscillator is also shown. As is evident, the slow switching circuit greatly attenuates the switching artifacts, lowering the switching noise by more than $10 \mathrm{~dB}$ close to the carrier, and largely suppressing it completely further away. The asymmetry of the spectrum is due to the fact that the spectrum is generated by a swept narrow band filter. Since the lower frequencies are generated first, the lower half of the spectrum is more representative of the earlier portion of the transient while the upper half represents the later portions. 


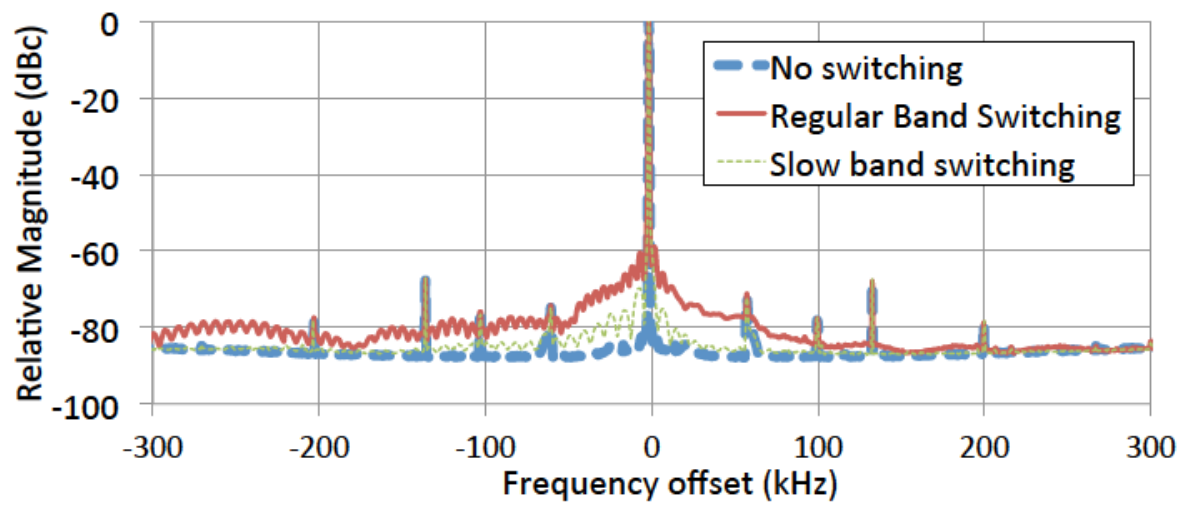

Figure 5.12: Plot of the average spectrum of the synthesizer output over a 1 minute period with no band switching, with regular band switches at random intervals, and with slow band switching at random intervals. 


\section{Chapter 6}

\section{Circuit Improvements}

The work discussed in chapters 3-5 described the theoretical behaviour underlying the concept of slowly switched capacitor banks to implement gradual frequency band switched with minimal transient effects, discussed the implementation of the circuits, and showed the measurement results.

While the measurements of the test circuit have demonstrated the principles of this concept, several additional improvements were developed to improve overall performance by reducing variability caused by supply voltage or temperature changes as well as susceptibility to power supply noise. These will be discussed and demonstrated in the following sections.

\subsection{Diode line calibration}

Section 3.1.3 showed that transistors can be biased in the sub-threshold region of operation with a line of diode connected transistors. However, this was shown to have a high sensitivity to process variation. In addition, simulation of the circuit (conducted in a similar BiCMOS process) showed that it was also very sensitive to supply voltage variations and temperature variations. This gives a wide range to the switching time of the capacitor bank, and makes it difficult to predict performance. While the test chip design attempted to overcome this issue by designing for the worst case corner, it is often desirable to be able to control the behaviour of the circuit.

The diode chain can be modified to be able to control the rise/fall time of the switching circuit and to reduce susceptibility to process variation, temperature drift or changes in supply voltage, while still retaining its full functionality. This can be done by biasing the diode line with a programmable current mirror, and adding switches to be able change the number of transistors in the diode line. A schematic of this can be seen in Fig. 6.1. In order to test this circuit, it was implemented in the NXP QuBiC 4XI 0.25 $\mu \mathrm{m}$ SiGe BiCMOS 


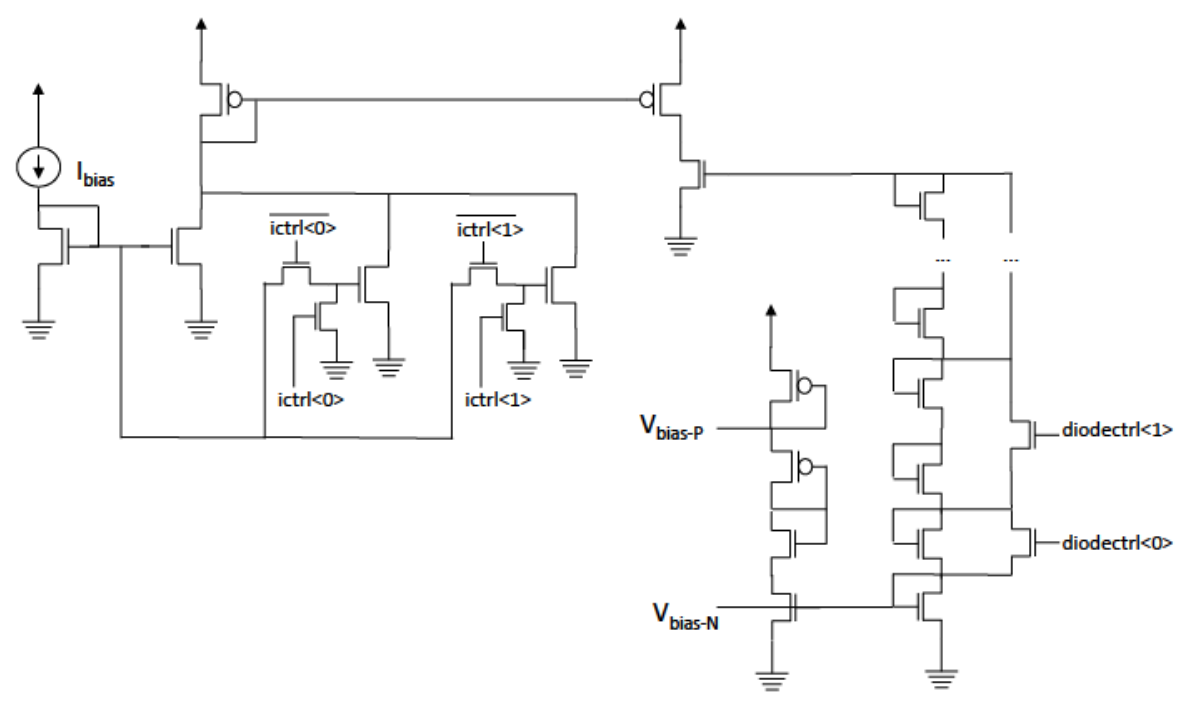

Figure 6.1: Schematic of a diode chain with programmable current mirror and switched diodes

process, as the IBM $0.18 \mu \mathrm{m}$ BiCMOS process used in the previous test chip was no longer available.

In order to determine which settings to use for the controls, a calibration circuit is required. Fig. 6.2 shows one possible circuit to calibrate the rise/fall time controls. A replica buffer with the sub-threshold bias transistors is implemented. A set of comparators can be used to detect low and high threshold crossings, so as to measure rise times (or fall times with slight modifications). Since the buffers are current limited by the sub-threshold transistors, the rise/fall times are much larger than the reference clock period, and thus the timer can be clocked using the reference oscillator.

A controller monitors the recorded rise/fall times for a given control setting and varies it as necessary. Upon start up, iterations can be performed to find the setting giving a rise/fall time closest to a specified target value. During operation, background calibration can be performed by noting if the rise/fall times from the current setting have drifted too far away from the target, and correct the settings as necessary.

The effect of the diode control lines with such a system can be simulated over different process corners with different temperature or supply voltages. Fig. 6.3 shows a plot of the rise/fall time of the buffer over 2 cases: one with a bias generated by a simple diode line, and another with a bias generated by the programmable diode line with calibration applied for each step. This is done over 3 process corners (fast, typical, and slow) and with different supply voltages. As can be seen, the high variability of the rise/fall times caused by process variation or supply voltage changes is greatly reduced. 


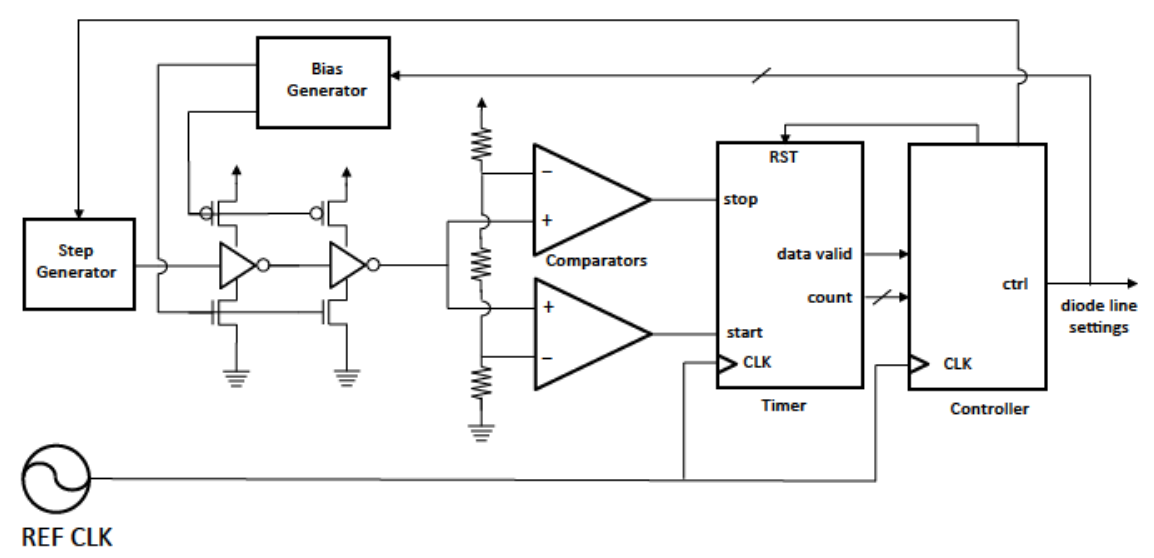

Figure 6.2: Schematic of a diode line calibration circuit

A similar result can be seen in Fig. 6.4, only with different temperatures instead. Again, the programmable diode line and feedback circuit allow the buffer performance to be controlled more carefully, although the range of the diode chain is limited at high temperatures wth the fast process and at low temperatures with the slow process. However, the range of the circuit can be increased by increasing the range of the current bias line.

\subsection{Control line dithering}

The previous section showed how modifications can be made to the diode line in order to compensate for process, voltage and temperature variations. However, even with the programmable diode line, the maximum rise/fall time is still heavily constrained by process parameters, since using a very small bias current runs the risk of allowing the circuit behaviour to be dominated by parasitics. The ability to further increase the switching time could further any transient effects of switching.

An alternative method to increasing the rise/fall time of the sub-threshold limited buffers is to apply a pulse-width modulated control signal with a gradually varying duty cycle, creating dithering. The slowly moving output of the buffers act as a low pass filter with a very low cut-off frequency, so the modulated control signal can be generated by the reference oscillator. To reduce spurs, the modulated control signal can also be generated using a $\Delta$ Emodulator. This essentially implements a DAC to control the switch voltage, but uses less complicated circuitry, due to the presence of the sub-threshold biased transistors which act as low pass filters, allowing the modulation to be done at a low clock rate.

Fig. 6.5 shows the transient buffer output waveforms with this. The diode line was 


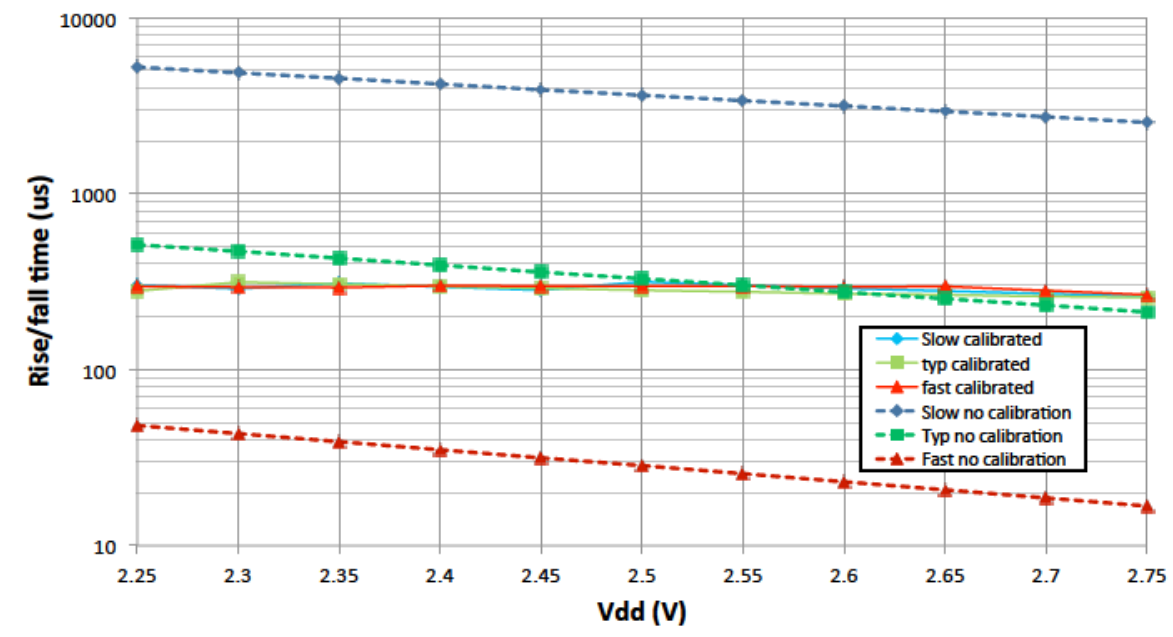

Figure 6.3: Plot of the rise/fall times of the sub-threshold limited buffers with different process corners and supply voltages both with and without calibration

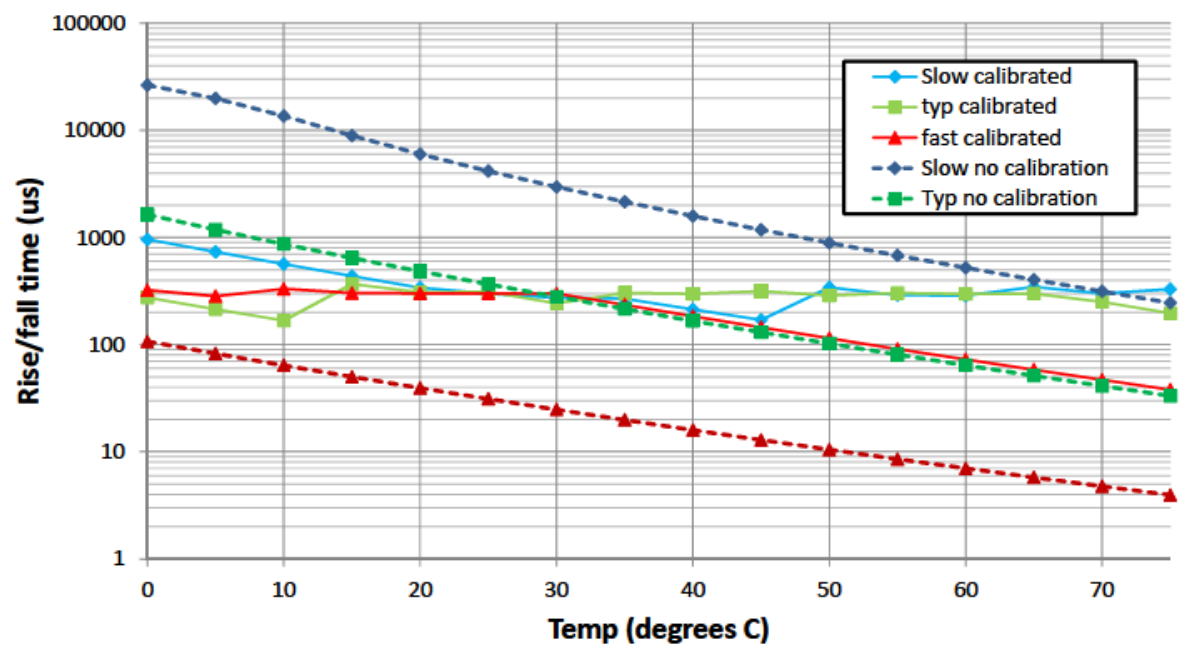

Figure 6.4: Plot of the rise/fall times of the sub-threshold limited buffers with different process corners and temperatures both with and without calibration 


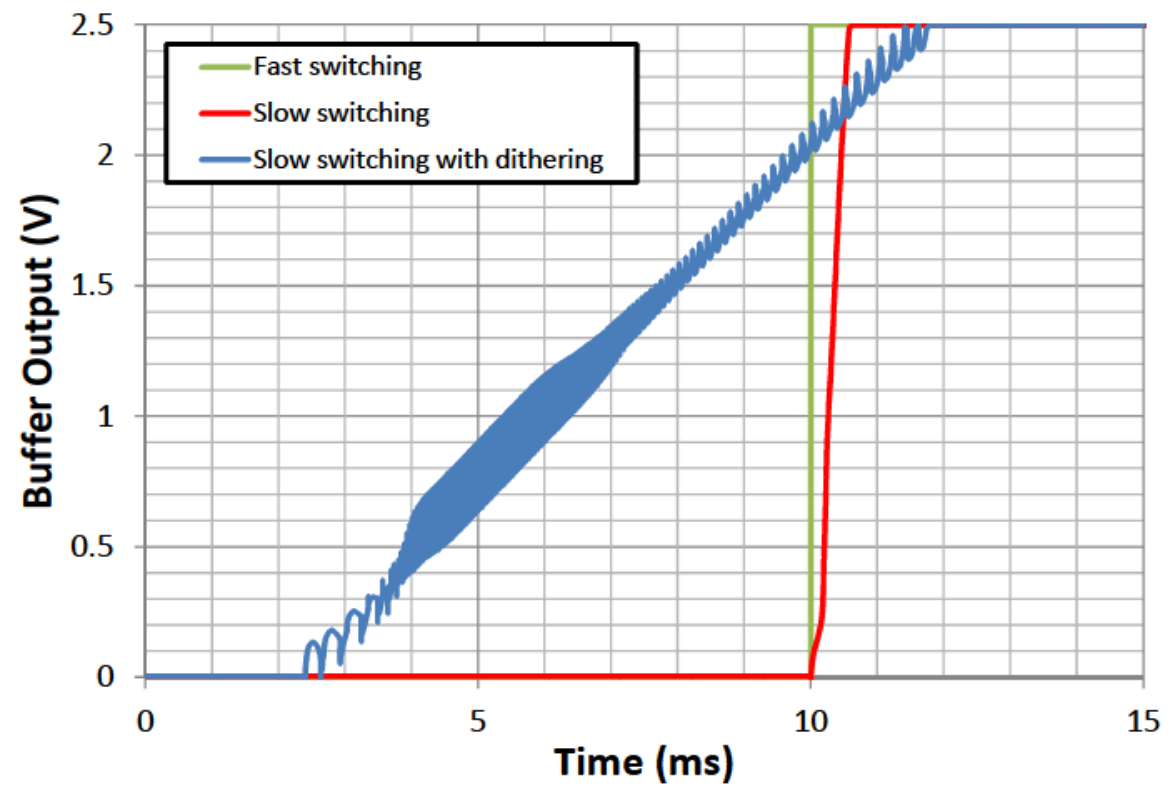

Figure 6.5: Plot of a buffer output

biased to set for a rise/fall time of $300 \mu \mathrm{s}$, while the input signal was a $200 \mathrm{kHz}$ pulsewidth modulated signal with a linearly varying duty cycle, which is also shown in the plot. For comparison, the transient waveform for the high speed switching case (with the subthreshold bias disabled) and for the slow switching case without dithering are also shown. As can be seen, adding the modulation allows the rise/fall time to be greatly extended. A more gradual input sequence can be used to further increase the rise/fall time easily, while a faster modulation rate can be used to reduce the transient noise (which was not done in this plot to reduce simulation time). The non-linear output characteristic of the buffer in relation to the input duty cycle arise due to the non-linear response the buffer has due to the different transistor regions of operation.

\subsection{Power supply noise rejection in complementary VCOs}

As described in section 4.10.2, despite the potential higher theoretical performance that the complementary cross-coupled VCO topology can offer, it generally performs poorly in realistic implementations due to the potential power supply noise coupling at the tuning port, which were found in simulation to be the overwhelmingly dominant noise source. Unlike the NMOS or PMOS implementations, the CMOS implementation does not provide a low impedance to both sides of the tuning element to the filter reference, which increases power supply noise seen there, which can then be up converted as phase noise. 


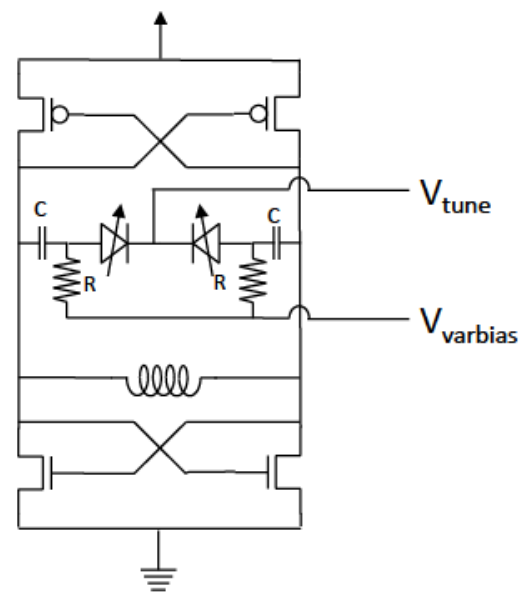

(a)

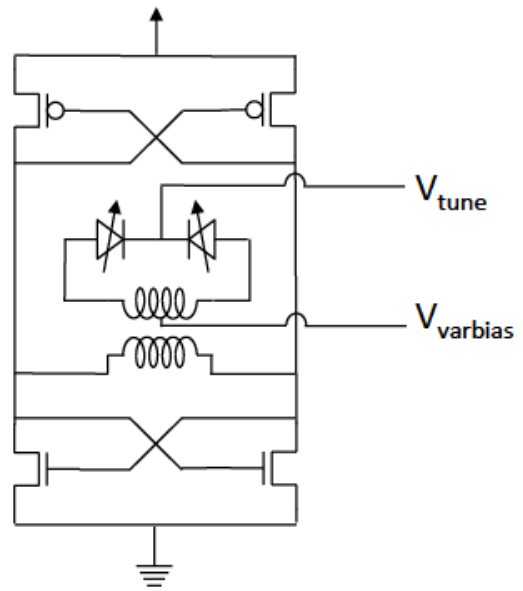

(b)

Figure 6.6: Simplified schematics of the proposed noise rejection CMOS VCOs

To reduce this problem, an additional low impedance path can be introduced to the filter so that power supply noise is shorted out. However, this must not have any impact on the DC current path between the complementary cross coupled pair, or this will adversely affect the performance of the oscillator. Two different circuit topologies are looked at to perform this. This can be seen in Fig 6.6, which shows simplified versions of the schematics without any bias circuits. In version (a), a capacitor separates the varactors from the drain nodes, adding a high impedance between the two at low frequencies, while still allowing the varactors to function properly at the resonant frequency. The DC bias of the varactors is controlled by a pair of resistors. From a supply noise perspective, this adds a high pass RC filter between the power supply and the varactor, which works to suppress the close in phase noise of the VCO. To maximize the benefit of this circuit, the cut-off frequency should be set high to suppress as much noise as possible. This requires small $\mathrm{C}$ and $\mathrm{R}$ values. However, the minimum value of $\mathrm{C}$ is set by the need for the VCO signal to couple from the tank to the varactors in order for them to be able to affect the frequency, while the minimum value of $\mathrm{R}$ is determined by the transconductance of the transistors. If it is too small, they will act as a short circuit and lower the quality factor of the circuit. However, a large $\mathrm{R}$ value is also detrimental since it will produce thermal noise and it maintains the high impedance seen at the varactor at this point, possibly allowing for other sources of noise to affect the VCO performance.

An alternative topology is explored in Fig 6.6 (b), which uses a balun or a transformer to couple the varactors to the VCO tank. This has several advantages: the transformer itself has 
a high pass characteristic, while the varactors are able to see a very low DC impedance. From a power supply noise perspective, the transformer only sees the differential noise voltage, which ideally would be close to 0 since the $\mathrm{N}$ and $\mathrm{P}$ paths are closely matched. Thus, the coupling of the common mode power supply noise to the varactor tuning port will be greatly reduced, reducing phase noise. However, a transformer requires more area or thinner lines to implement, and is more difficult to characterize electro-magnetically, which is critical since the resonator plays an important role in the performance of the oscillator.

The use of transformer based VCOs is not unprecendented. Transformers have been used in LC resonators to improve quality factor [200-202], to very the signal level at the gate vs the output [203], for back gate biasing [204], for quadrature coupling [205,206] or vary the output common mode voltage [207]. Reference [177,208] uses a transformer to form the basis of a dual band oscillator, by taking advantage of multiple resonant nodes present in the VCO, while $[209,210]$ uses a transformer to implement a differential tuning port, and [211] uses a transformer to increase the third harmonic components present at the resonator to improve the noise impulse sensitivity function.

While the approach shown here is similar to those shown in $[209,210]$ (since the $\mathrm{V}_{\text {tune }}$ and $\mathrm{V}_{\text {bias }}$ could be considered to be a differential tuning voltage), the approach shown here still only implements a single ended tuning port (which makes charge pump and loop filter design easier), relaxing constraints for identical impedances seen at both ports. As a result, the design is simpler, and the less complex varactor and transformer structures could result in improved quality factor and/or tuning range.

\subsubsection{Noise Analysis and Simulation}

Small signal analysis can be performed on the two noise rejection topologies to demonstrate how the path allowing power supply noise to couple to the varactor is attenuated. Fig. 6.7 shows a simplified model of the RC coupled VCO (without biasing circuitry), showing the common mode path from the voltage supply noise source to the varactor. As can be seen, the RC circuit places a high pass RC filter. Performing small signal analysis on this gives a transfer function from the noise voltage $V_{n}$ to the varactor voltage $V_{v a r}$ as follows:

$$
\frac{V_{v a r}}{V_{n}}=\frac{j \omega R C g_{m p}}{1+j \omega C\left(R+\frac{1}{g_{m p}+g_{m n}}\right)}
$$

This shows that the noise is attenuated below a cutoff frequency of $C\left(R+\frac{1}{g_{m p}+g_{m n}}\right)$.

Fig. 6.8 shows a simplified model for the transformer coupled VCO (again without the biasing circuitry). The mismatch between the two sides of each differential pair is modelled by a resistor $R_{\Delta}$. The transformer is modelled as 2 impedances and a voltage controlled 


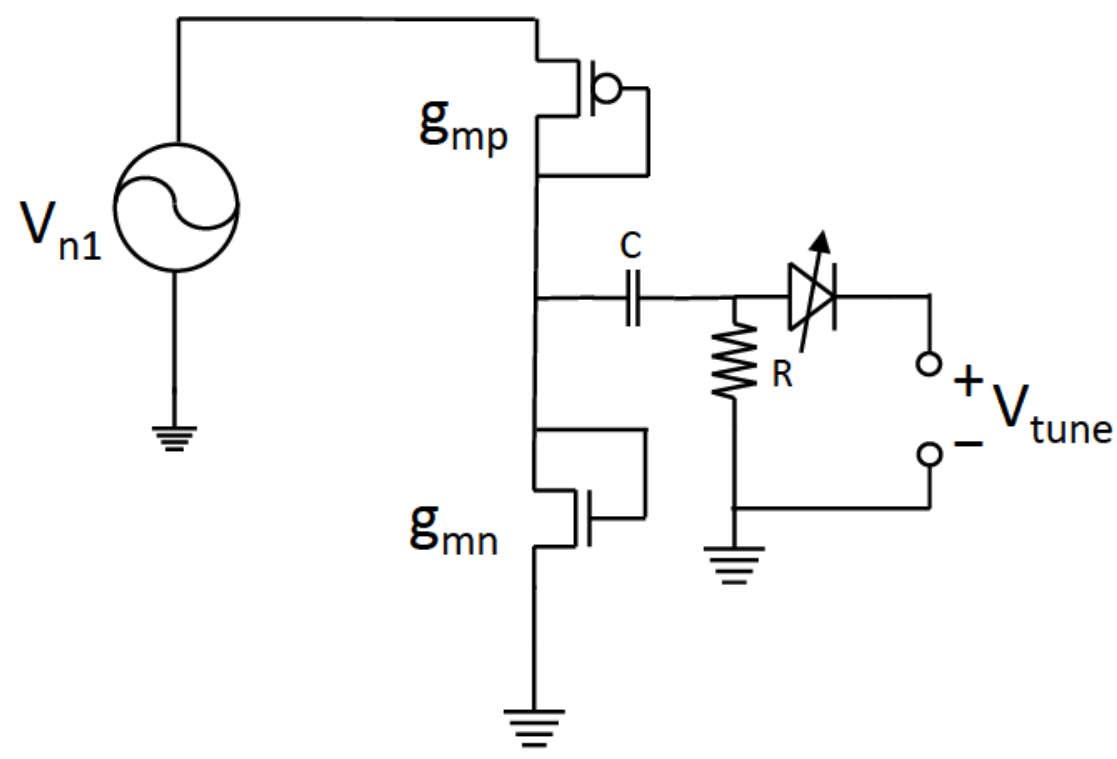

Figure 6.7: Simplified small signal model of the RC noise rejection VCO

voltage source with a gain of $\mathrm{k}$, whose value is determined by the frequency dependant coupling factor. With this, the following noise transfer function from the noise voltage $V_{n}$ to the varactor voltage $V_{v a r}$ can be derived:

$$
\frac{V_{\text {var }}}{V_{n}}=\frac{k}{2} \frac{g_{\Delta} Z_{\text {prim }}\left(1+g_{m n} Z_{\text {prim }}\right)}{Z_{\text {prim }}\left(2+g_{m p} Z_{\text {prim }}+g_{m n} Z_{\text {prim }}+g_{\Delta} Z_{\text {prim }}\right)\left(g_{m p} R_{T}+g_{m n}\right)}
$$

The impedances of the primary and secondary windings are primarily resistive at lower frequencies, and thus, the numerator of the transfer function consists of 2 very small terms multiplied by each other (the mismatch between the two sides and the resistance of the
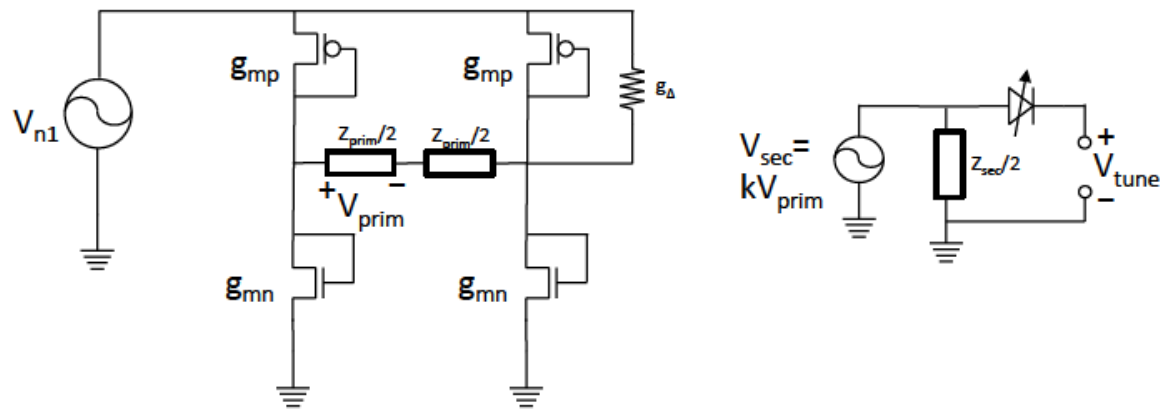

Figure 6.8: Simplified small signal model of the transformer noise rejection VCO 


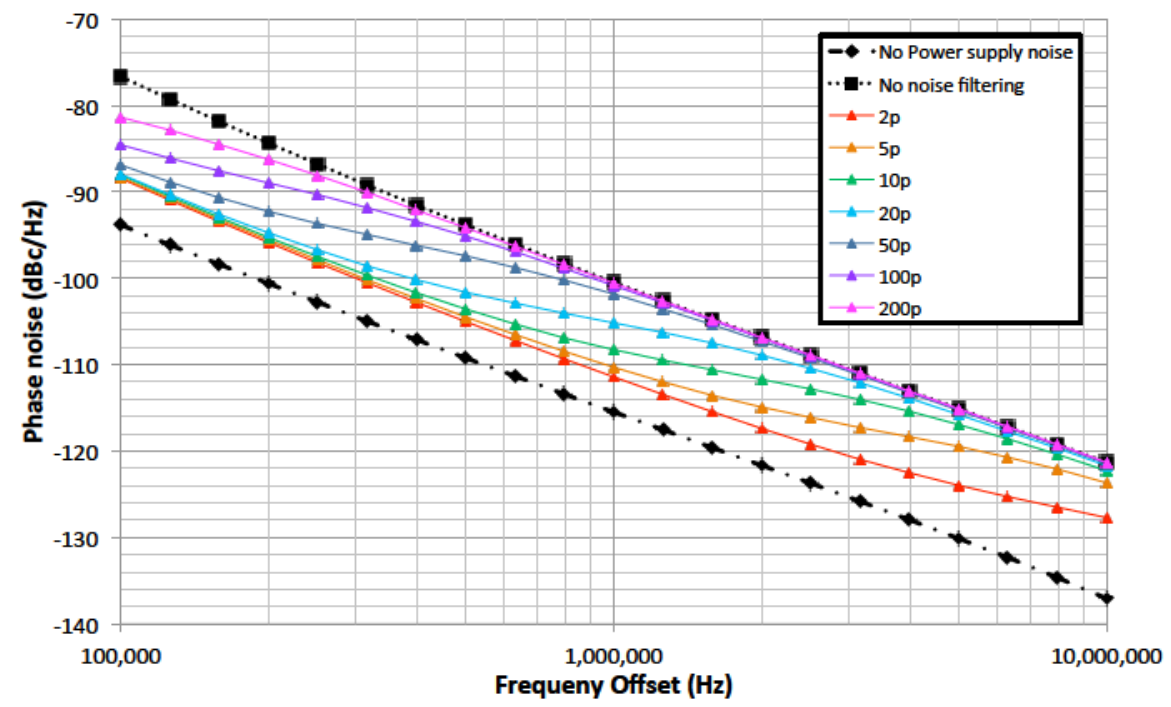

Figure 6.9: Simulated phase noise of a VCO prototype showing the effect of power supply noise on the $\mathrm{RC}$ noise rejection $\mathrm{VCO}$ phase noise

primary windings). This shows that, unlike the RC noise rejection VCO, the transformer has strong wide band noise rejection that can be reduced by good matching between the two sides of the cross-coupled pairs and low series resistance in the primary windings, both of which are already necessary for good VCO performance.

To simulate the RC filter based noise rejection oscillator, a VCO using the RC filtering topology was simulated, along with a comparison version without the $\mathrm{RC}$ filter. The circuit was simulated using variable filter bandwidths by varying the capacitance value. Fig. 6.9 shows the phase noise for these cases, as well as with the case of no power supply noise, and the case with no noise filtering. As can be seen, the noise filter allows power supply noise to be rejected up to the filter bandwidth. While this is able to reduce the phase noise by up to $10 \mathrm{~dB}$, this does not take the oscillator close to the value with no power supply noise at all, since this circuit only removes the noise coupling to the varactor line. Other paths allowing power supply noise to be up-converted as phase noise exist, particularly the gate-source capacitances of the cross coupled pairs. Thus, further work on will be needed to determine how to eliminate the effect of this.

A similar simulation was conducted to simulate the transformer VCO topology. Fig. 6.10 shows the phase noise for this circuit for the case with and without supply noise, as well as for a similar VCO with supply noise and without any noise filtering. The transformer noise rejection topology again is able to reduce the phase noise by $10 \mathrm{~dB}$, but is not able to eliminate the effect completely. Unlike the RC topology, the noise rejection is done over a broad frequency range, since there is no filter bandwidth limiting its effect. 


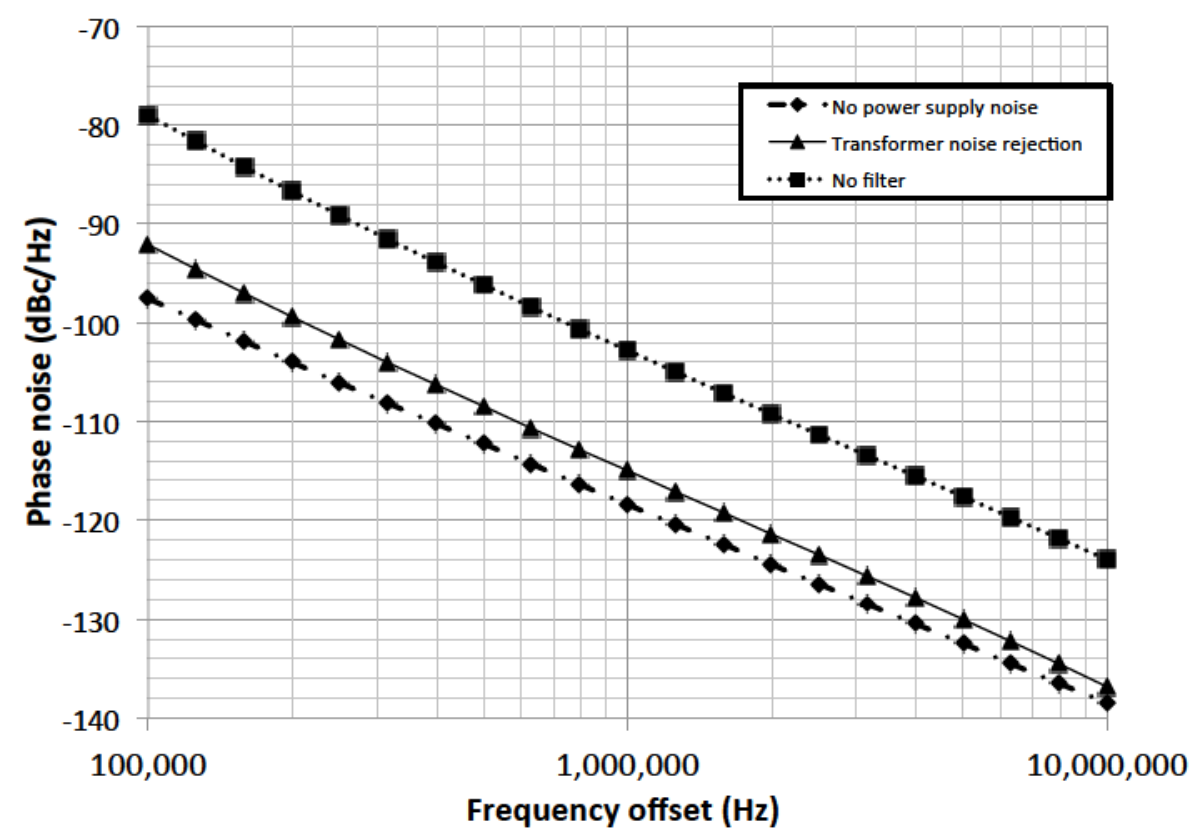

Figure 6.10: Simulated phase noise of a VCO prototype showing the effect of power supply noise on the transformer noise rejection VCO phase noise

\subsubsection{Test chip architecture and specifications}

The design of the test chip is to look at the previous concepts for the noise rejection VCOs. To simplify the testing and design process, the VCOs will be tested unlocked so that a PLL is not needed. Due to area limits set by the foundry and manufacturing partners, sufficient area was given to build 3 different VCO variations. A set of specifications was then derived for the test chip:

- the chip should include 3 separate VCOs, isolated from each other so that each operates independently. Each can be tested on its own, relaxing pad frame constraints.

- center frequency around $4 \mathrm{GHz}$, with at least $500 \mathrm{MHz}$ of tuning range. This shows the validity of the concept in a circuit operating at radio frequencies with a practical tuning range, while remaining within the useful range of the process. It would be reasonable to expect to be able to scale the frequency of the oscillator to slightly higher frequencies and lower frequencies with expected performance tradeoffs as well in order to accommodate different applications

- the tuning range should be achieved with 15 switched capacitor banks and 1 fine tune varactor 
- the VCOs should have a large output signal swing (-10 to $0 \mathrm{dBm}$ after buffering) for more accurate phase noise results

- the chip should use multiple $1.2 \mathrm{~V}$ supplies, with separate supplies for all test VCOs and buffers

- the chip should have external controls for capacitor banks, and varactor bias ports

\subsubsection{Process}

The process used was the IBM $0.13 \mu \mathrm{m}$ CMOS $8 \mathrm{RF}$ process. It uses 8 metal layers (with the upper two metal layers being thick copper and aluminum layers), 1 poly layer, and support for NMOS and PMOS with minimum drawn gate lengths of $120 \mathrm{~nm}$. The NMOS transistors have a simulated maximum $\mathrm{f}_{\mathrm{T}}$ of around $100 \mathrm{GHz}$ using the process kit $\mathrm{RF}$ models. The process has support for resistors (using silicided, non-silicided and thin film resistors), capacitors (AMOS varactors with grounded bulk, MIM capacitors and dual MIM capacitors) as well as inductors using the top two thick metal layers.

\subsubsection{Shared test circuitry}

Digital controls: Although the I2C interface used in the PLL test chip was highly effective in the testing process, a macro for the receiver was not readily available in the $0.13 \mu \mathrm{m}$ process. Thus, the on-chip registers were set using a set of shift registers controlled using 3 pins: 1 pin contained the data, the other a clock signal to shift in the data into the shift register, and a final pin to control a strobe setting to change the data on the shift registers onto the registers actually controlling the oscillators.

Buffers: The output signals of the VCOs were connected to open drain NMOS buffers driving the pads, transmission lines and cables, and the output loads. The buffer schematic is as shown in Fig. 6.11, which also shows the proposed test setup for the chip. It consists of an NMOS transistor with its gate connected to the VCO tank (or the output of another buffer), its source grounded, and the drain connected to the output pad. During testing, this pad will be connected to a bias-T, with the DC controlling the DC bias of the buffer and the signal either being fed into the measurement equipment or being terminated. This circuit was chosen for its ease of implementation, and high gain and high output power with only 1 buffering stage with a very simple bias structure that still gives control over the output signal level. It's major drawback, high non-linearity, is not a major issue in the VCO testing process. Fig. 6.12 shows the simulated output and DC power for buffer with different buffer supply voltages. 


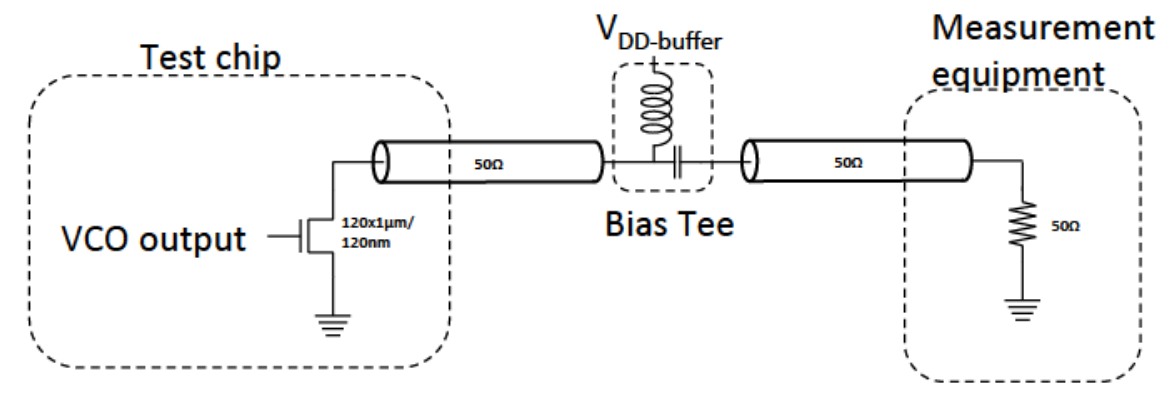

Figure 6.11: Schematic of the open drain VCO buffer connected to the test setup

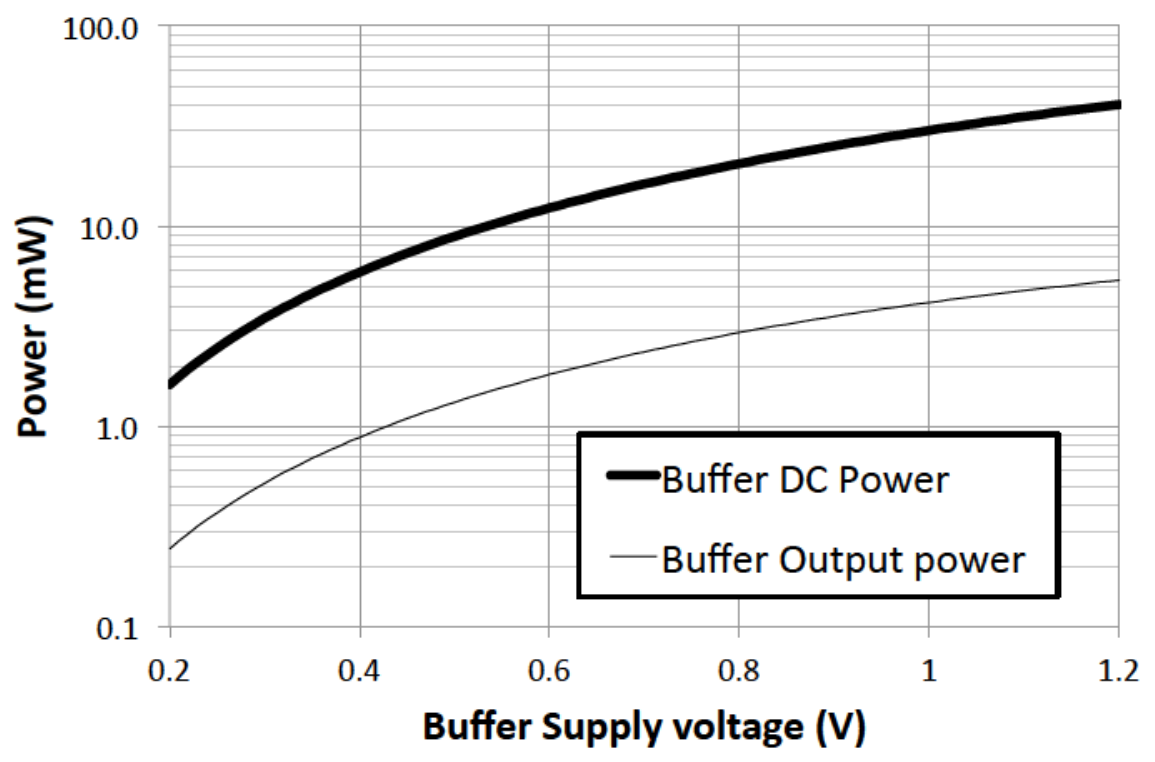

Figure 6.12: Simulation results of the oscillator buffer 


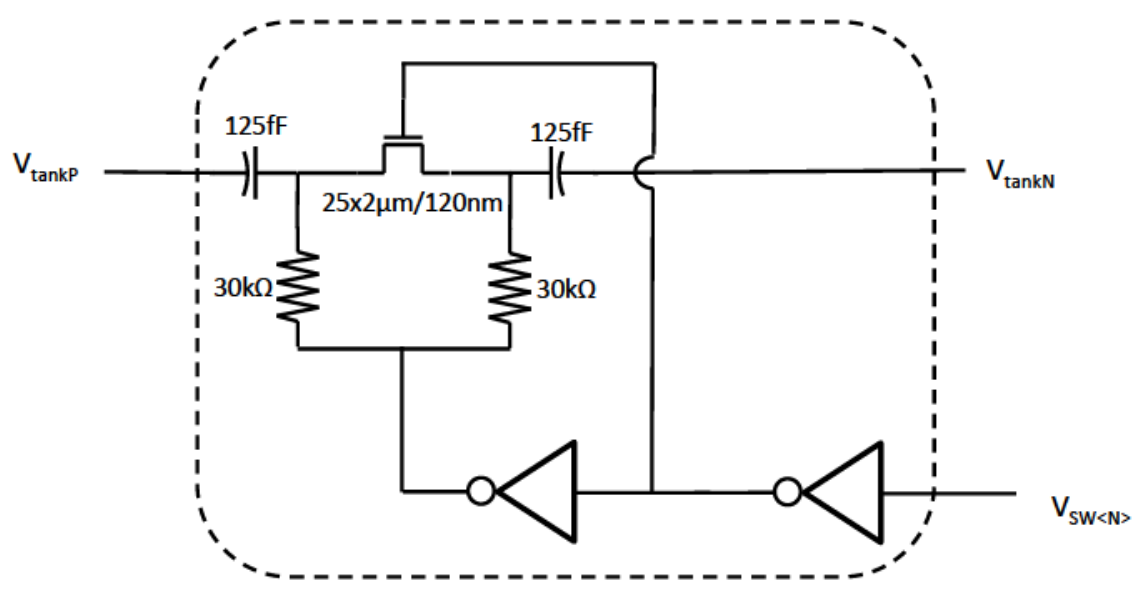

Figure 6.13: Schematic of the VCO capacitor banks

Capacitor and varactor banks: To minimize design time and to maximize the degree to which the different oscillators can be compared, identical capacitor banks and varactor banks are used for each of the 3 test oscillators. The schematic is as shown in Fig. 6.13.

\subsubsection{Oscillator variants}

As described previously, 3 different VCOs were tested on the noise rejection oscillator test chip. 2 versions were based on the RC coupling method and 1 version was based on the transformer based coupling circuit. The following sections describe the 3 different versions implemented on the chip

\subsubsection{Version 1}

Version 1 was based on the RC coupling method shown in Fig 6.6 (a). The full schematic is shown in Fig. 6.14, where it can be seen that it is similar to the circuit shown in Fig 6.6 (a), although it uses supply and ground inductors. This allows for some noise filtering, but mainly allows for larger signal swing, since the voltages of the sources of the NMOS and PMOS cross-coupled pairs to rise above the power supply and below the ground voltage. Since, there is no biasing circuit, the operating point of the circuit must be controlled by adjusting the power supply of the oscillator. The design was done by optimizing the size of the inductors and transistors using the Cadence Spectre RF simulation, using RF transistor models (which are reported to include wiring and via parasitics), as well as back annotated parasitic estimates for the resistance from the cross coupled pairs to the tank inductors and the capacitance between the two tank nodes. 


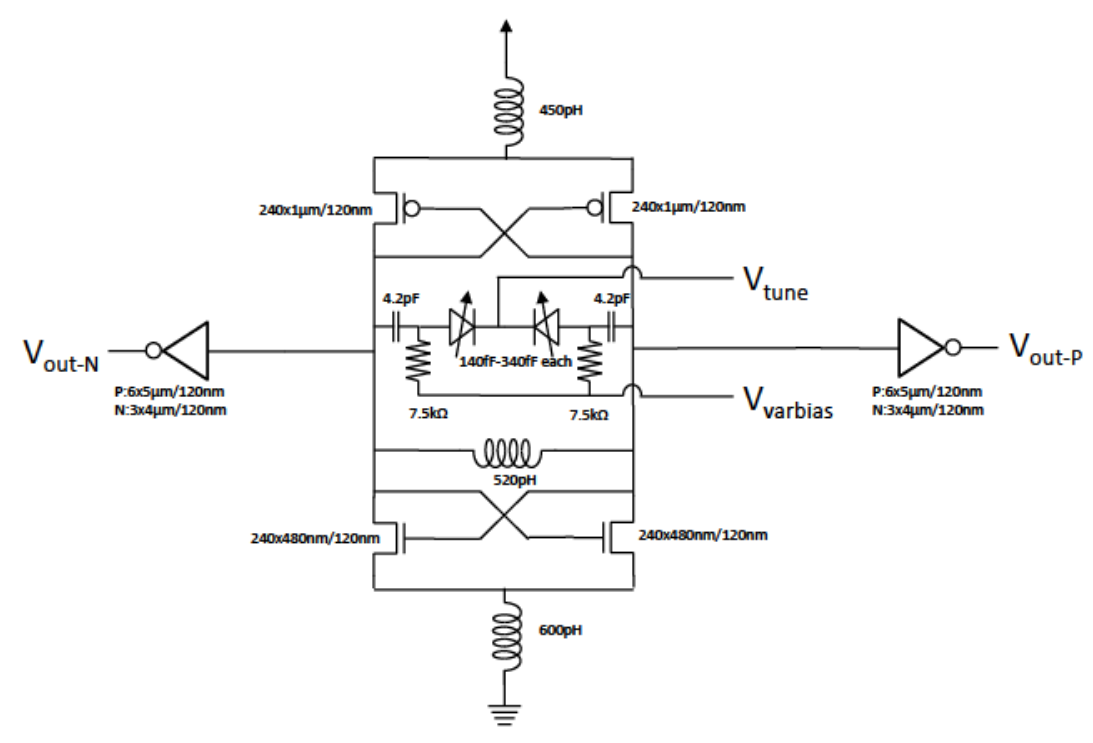

Figure 6.14: Schematic of the first VCO variation

Due to the large signal swing, a MOS inverter is used as a pre-driver before the main buffer. This reduces the capacitive loading on the tank, and allows for a smaller cross-coupled pair, reducing power consumption.

The inductor used was a single turn, $300 \mu \mathrm{m}$ diameter, inductor with $25 \mu \mathrm{m}$ wide turns. This geometry was chosen as it has a high quality factor with a moderately low inductance, allowing a higher tolerance for parasitic capacitance in the capacitor bank, allowing for larger switch transistor sizes to reduce the losses in the capacitor bank. The results of the inductor characterization simulations can be seen in Fig. 6.15.

\subsubsection{Version 2}

Version 2 was based on the RC coupling method shown in Fig 6.6 (a). The full schematic is shown in Fig. 6.16, where it can be seen that it is similar to that shown in version 1. However, since version 1 relies heavily on the accuracy of the simulation results using the models provided, an additional version using a tail biasing transistor was also implemented, which would have more robustness against process variation and give an additional control by which the performance of the circuit can be tuned. This tail transistor can operate as a current source, but is intended to operate in the triode region of operation so as to implement a tail resistor. The use of a tail resistor instead of a current source allows for higher current to be drawn during the oscillation crossovers, resulting in faster crossover, and improving the oscillator impulse sensitivity function, reducing phase noise [189]. 


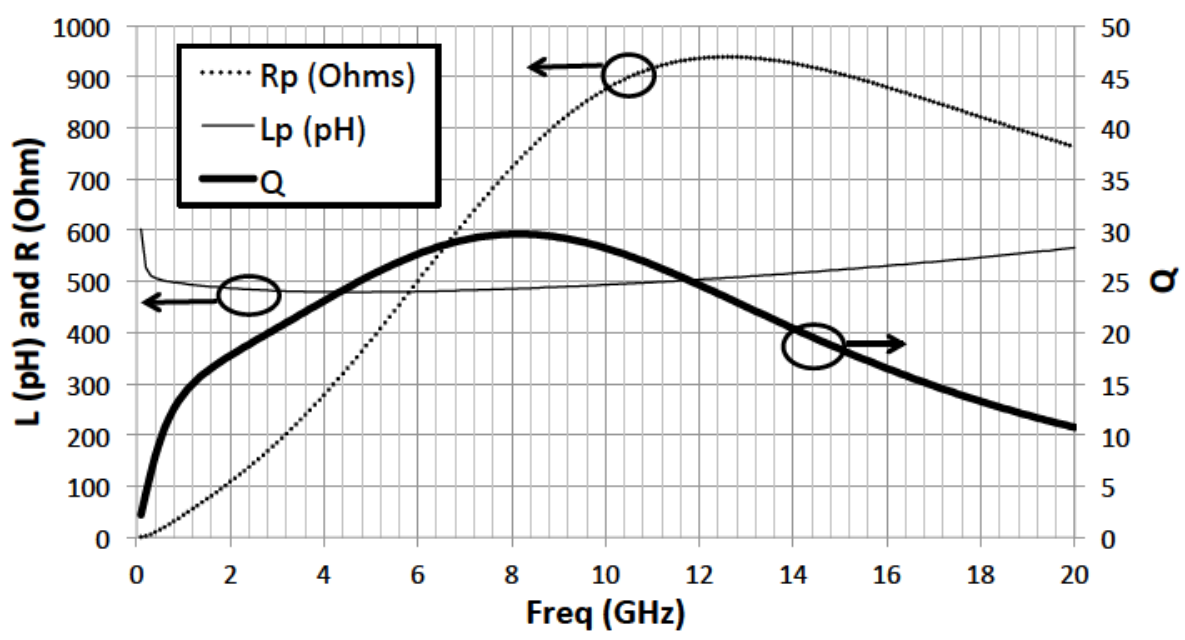

Figure 6.15: Schematic of the first VCO variation

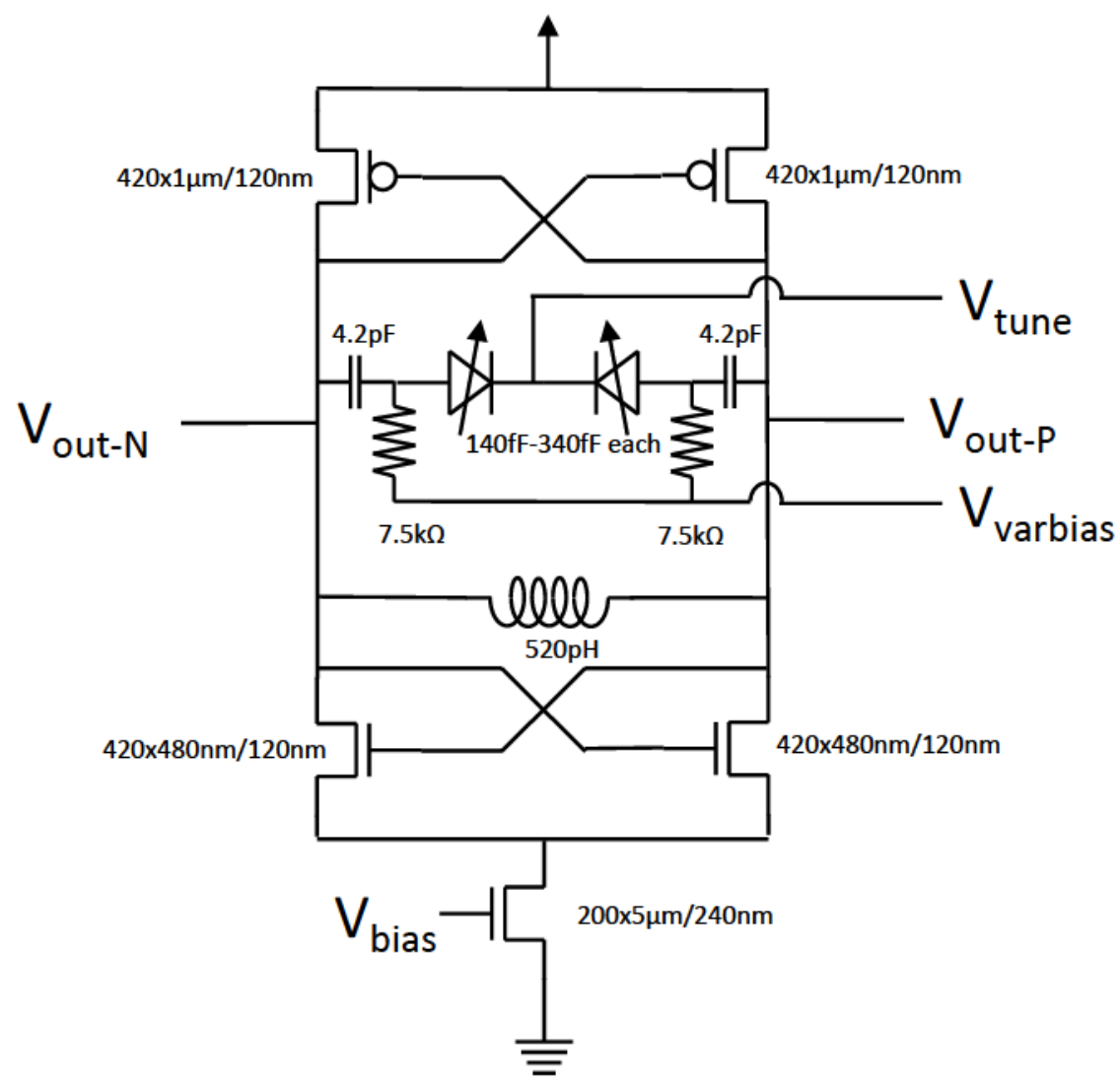

Figure 6.16: Schematic of the second VCO variation 


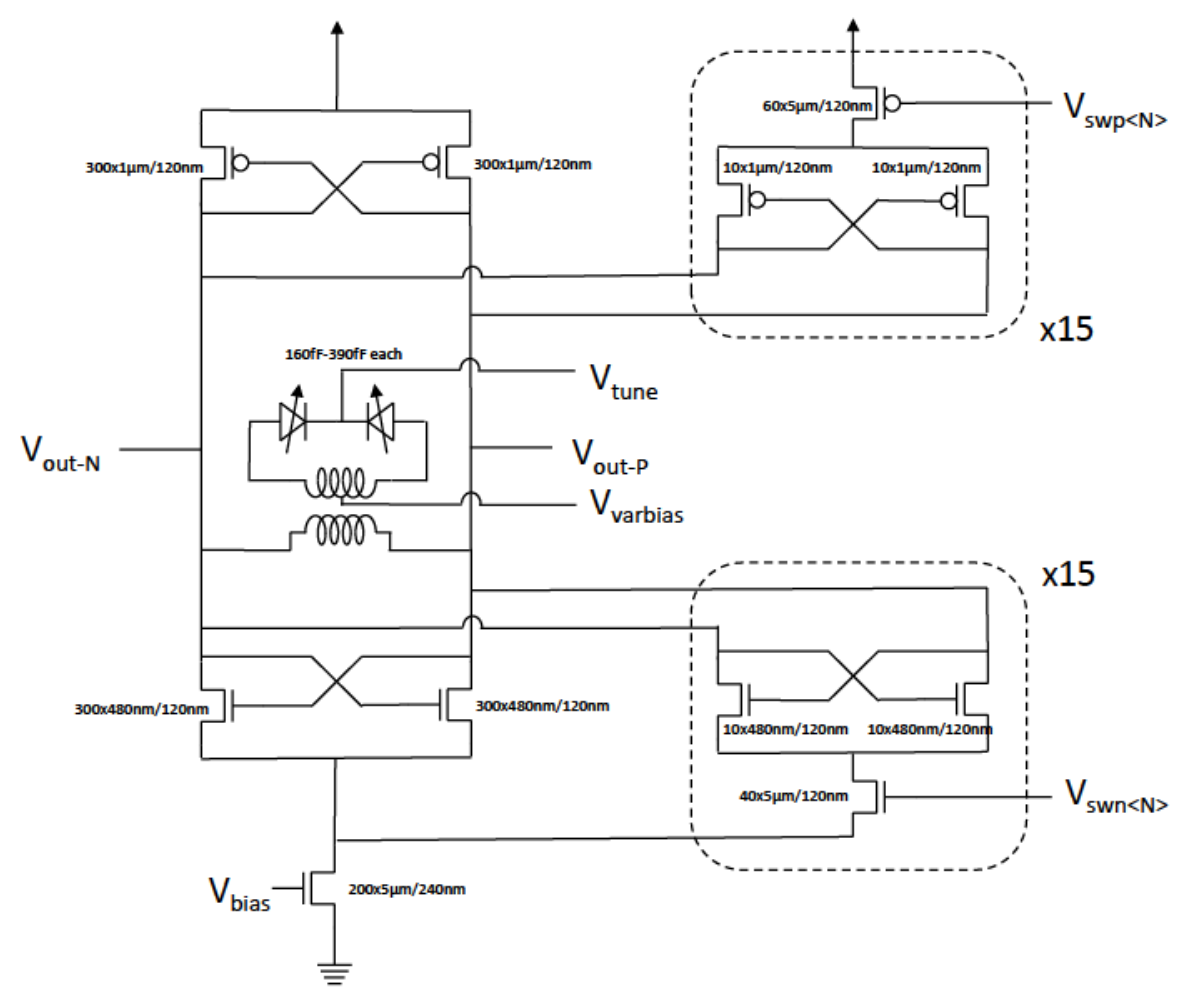

Figure 6.17: Schematic of the third VCO version

\subsubsection{Version 3}

Version 2 was based on the RC coupling method shown in Fig 6.6 (a). The full schematic is shown in Fig. 6.17, where it can be seen that it is similar to that shown in version 2 except with the transformer varactor coupling. Extra NMOS and PMOS cross coupled pairs were made available to be switched in due to potential inaccuracies in the transformer EM model, since it was custom created, allowing for control over the gain and for control over the NMOS/PMOS ratio.

\subsubsection{Transformer layout and characterization}

The layout of the transformer used is shown in Fig. 6.18. The design, which is $300 \mu \mathrm{m}$ in diameter with $15 \mu \mathrm{m}$ wide lines at a spacing of $5 \mu \mathrm{m}$, is based on the high-Q inductor used in the second oscillator test, but with thinner lines for both coils. To reduce series resistive losses, each of the two windings use the top two metal layers in parallel. This strategy reduces coupling between the windings since the side-wall capacitance is much smaller than the overlap capacitance between the two metals. 


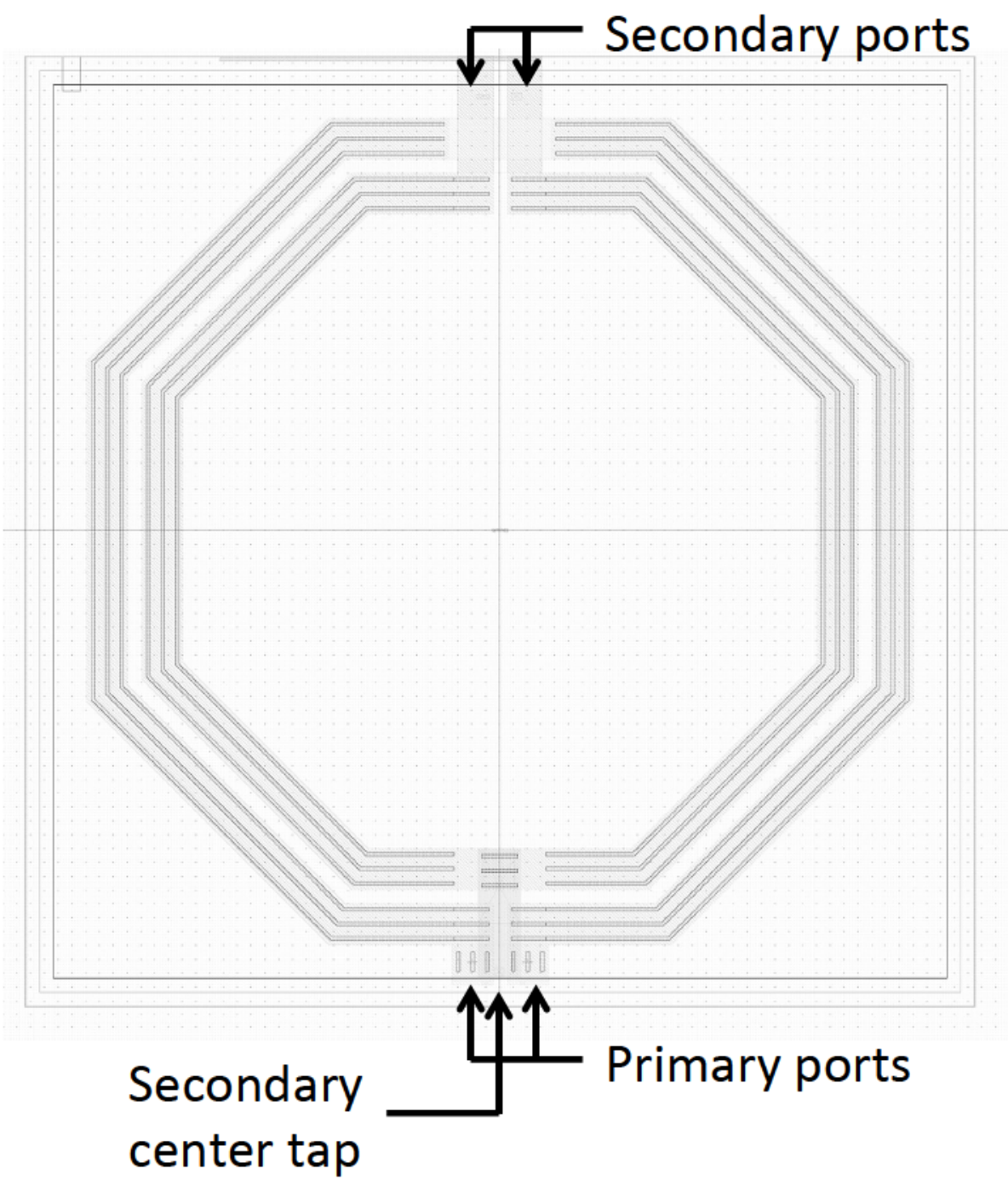

Figure 6.18: Layout of the transformer. The width and height are each $300 \mu \mathrm{m}$ 


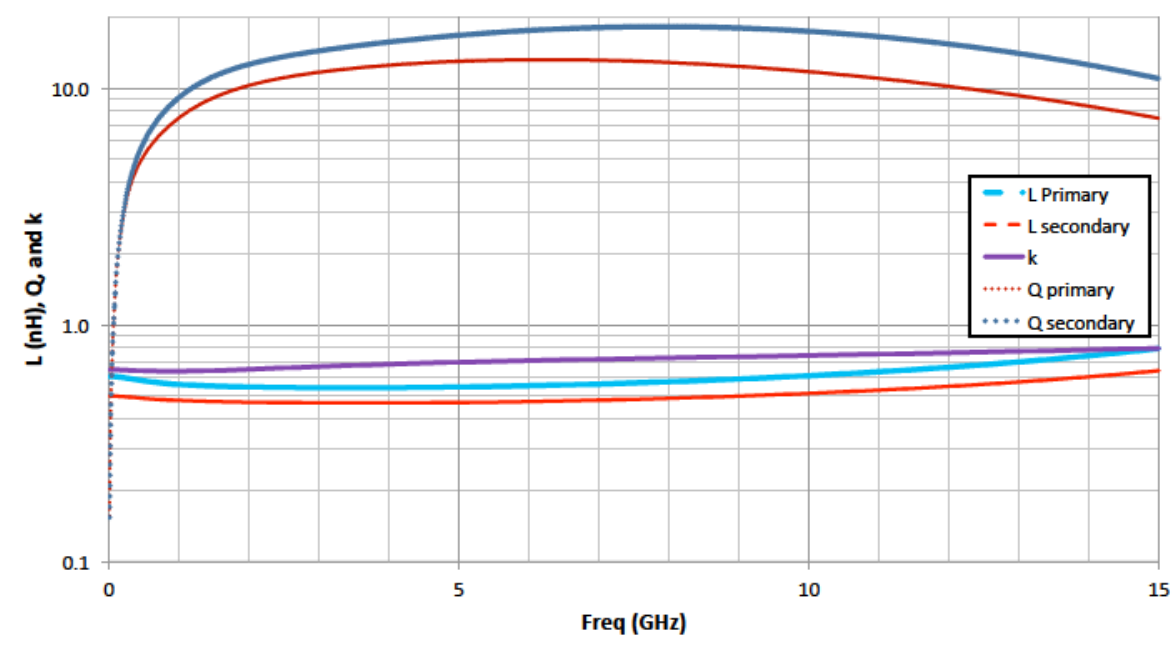

Figure 6.19: Plot of the transformer characterization simulation results

To simulate the transformer in the VCO circuit, a transformer model was synthesized. This was done to reduce simulation issues encountered when performing the transient simulations with a frequency domain model. This was done using Integrand EMX to simulate the transformer and construct the model, which consisted of lumped passive elements, which guarantees model causality. A plot of the transformer model simulation results can be seen in Fig. 6.19, showing the primary and secondary inductance, quality factor and coupling coefficients.

An important factor when dealing with transformer based oscillators is the possibility of multiple oscillation modes $[208,212]$. The possibility of this is reduced since a low turns ratio is used, greatly increasing the frequency of higher-order modes. The s-parameters of the transformer with the varactors and capacitor bank are simulated, with the real and imaginary impedances shown in Fig. 6.20, which shows only a single resonant point at the intended oscillation frequency. A potential higher order mode exists at $43 \mathrm{GHz}$, where the imaginary impedance approaches 0 and the real impedance increases slightly. However, the quality factor is very low and the circuit will not have gain at this frequency, preventing oscillation.

\subsubsection{Test chip layout}

The design of the circuit was laid out and a the full layout of the test chip was created. Fig. 6.21 shows the layout of the test chip. The different VCO versions are labelled, as well as the digital registers. The total active area required (excluding power supply decoupling) for the oscillators are $0.29 \mathrm{~mm}^{2}$ for version $1,0.25 \mathrm{~mm}^{2}$ for version 2 , and $0.31 \mathrm{~mm}^{2}$ for 

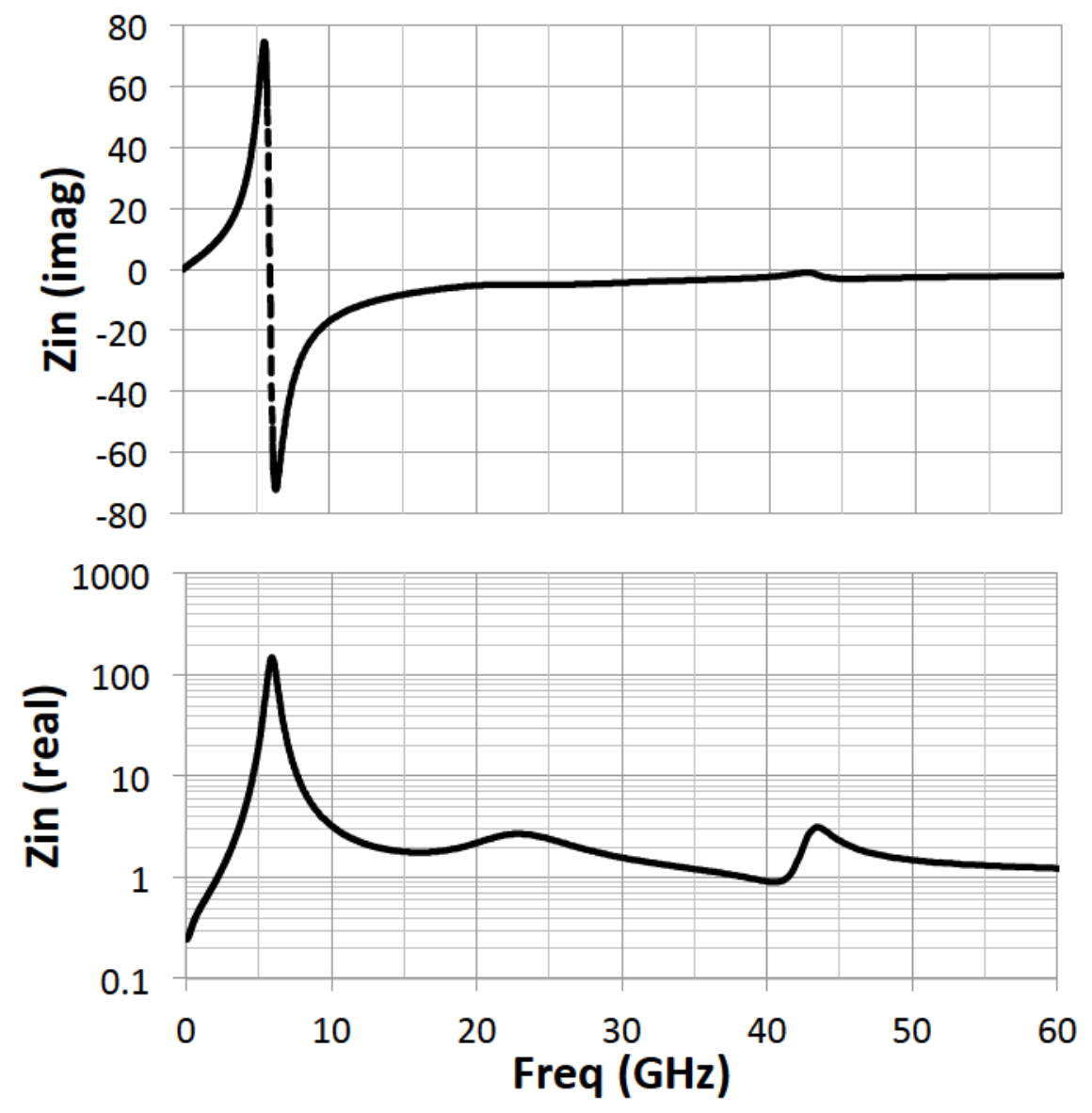

Figure 6.20: Simulated resonator impedance (real and imaginary) for the transformer coupled VCO 


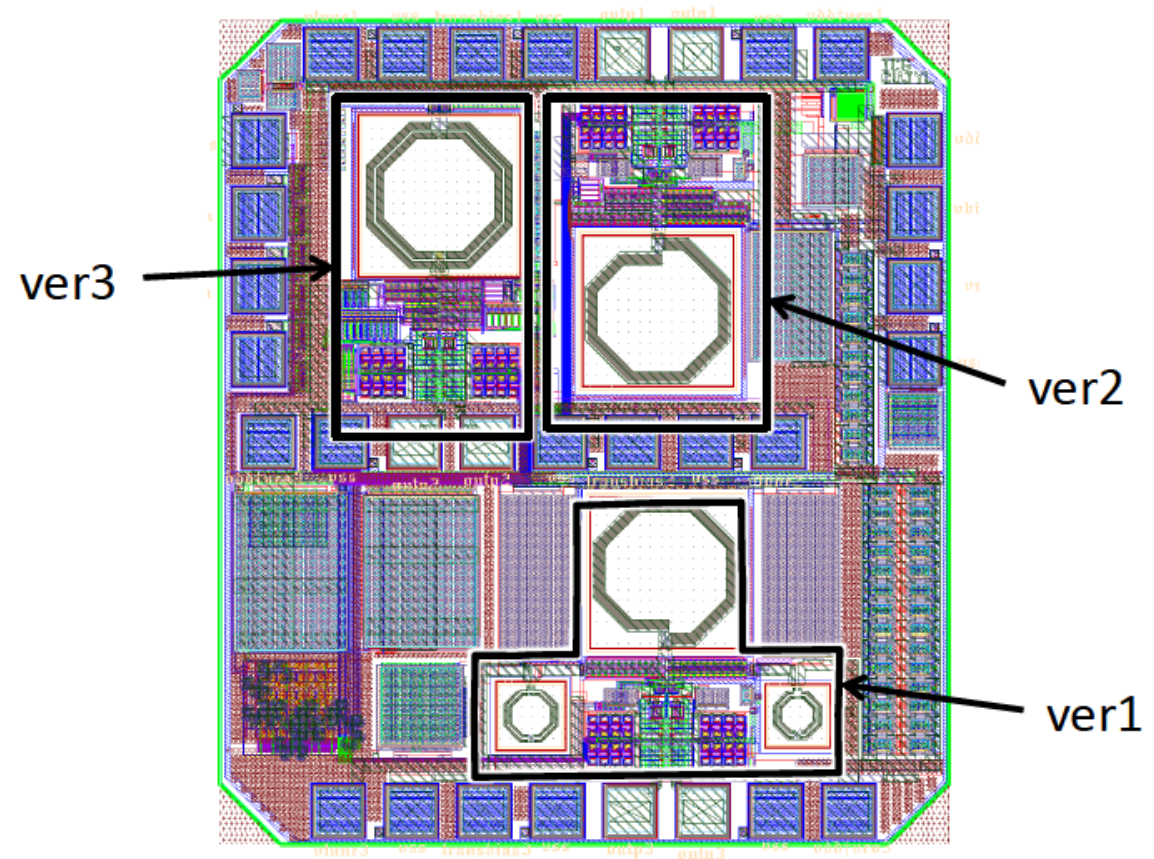

Figure 6.21: Layout diagram of the test chip

version 3 .

\subsubsection{Simulated frequency range, phase noise and power consump- tion}

All 3 of the oscillators were simulated at their optimal bias points. Fig. 6.22, 6.23, 6.24 and 6.25 show the frequency tuning range, phase noise at a $1 \mathrm{MHz}$ offset, DC power consumption, and figure of merit respectively over the entire range of the VCO. The simulations were conducted with a typical corner at a temperature of 75 degrees $\mathrm{C}$. The parasitics were modelled through the use of schematic back annotation based on parasitics derived from Diva extraction run, and resistances calculated using the process kit metallization resistance formulas. Output bond wire parasitics were based on simulations conducted in HFSS on a test bond wire structure. Since the measurements of the original PLL test chip showed how critical resistive losses were, wiring resistance was calculated and back annotated in multiple areas. The area with maximum sensitivity to resistive losses was found to be the connection between the gain transistors and the resonator. To reduce this as much as possible, thick metal lines were used, using the top metal layers when possible due to their lower sheet resistance. Large via connections were also used to help reduce vias resistance.

While the effect of many of these parasitics can be taken into account more accurately 


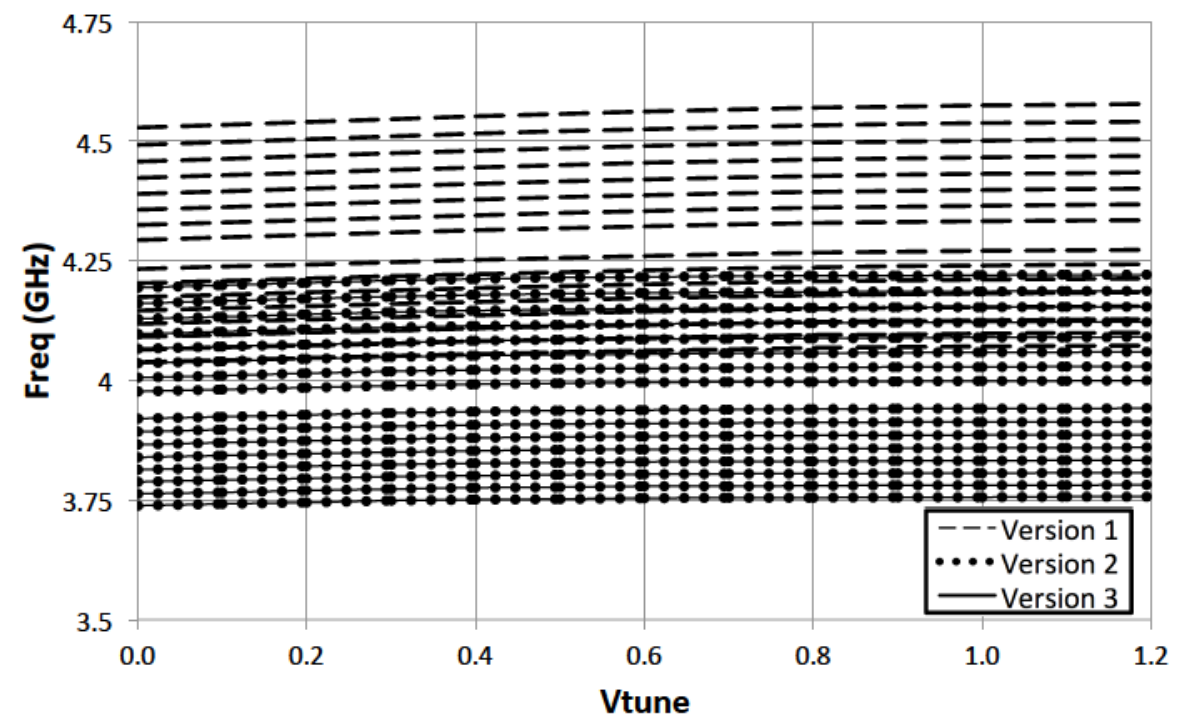

Figure 6.22: Simulated frequency tuning range of the three oscillator versions

by running a simulation of the extracted layout, a back annotated approach was used due to the much faster simulation speed. Since the performance of the VCOs heavily depends on the bias condition, many simulations would be needed to be run in order to determine the optimal bias conditions, which would take a prohibitively long time with a fully extracted layout.

The ability for these oscillators to reject power supply noise coupling to the tuning varactor was simulated by performing an $\mathrm{AC}$ simulation and looking at the gain from an $\mathrm{AC}$ source placed on the power supply to one of the varactors. The results are shown in Fig. 6.26 The third version (which uses transformer varactor coupling) is also simulated with a $100 \Omega$ offset resistor. As is evident, the RC coupled versions (first and second version) achieve high noise attenuation for low frequencies, but quickly rises until the $10 \mathrm{MHz}$ corner is reached, which the transformer version achieves good wide band noise rejection; enough that other minor parasitics not modelled here will likely dominate. The difference between the first and second version arises from the tail bias transistor in the second version, which increases the effective impedance to ground.

\subsection{Summary}

This chapter has shown some of the circuit improvements that have been developed to improve the performance of the circuits discussed in the previous chapter. The use of a calibrated, programmable diode line allows the rise/fall time to be adjustable, also allowing 


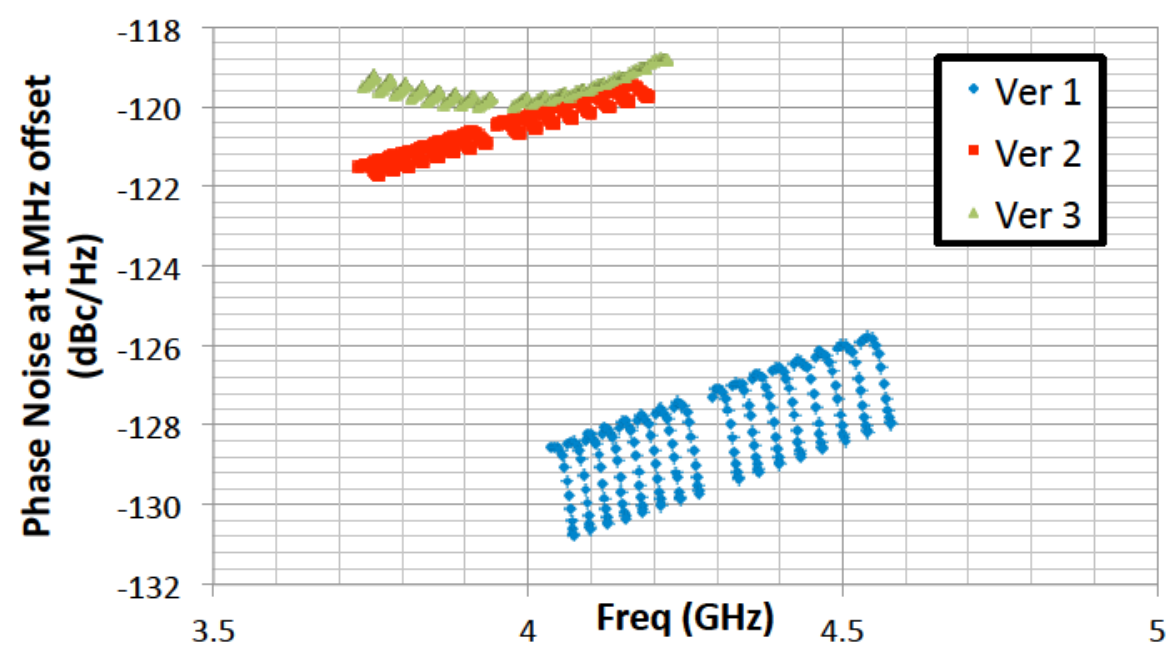

Figure 6.23: Simulated phase noise (at a $1 \mathrm{MHz}$ offset) of the three oscillator versions

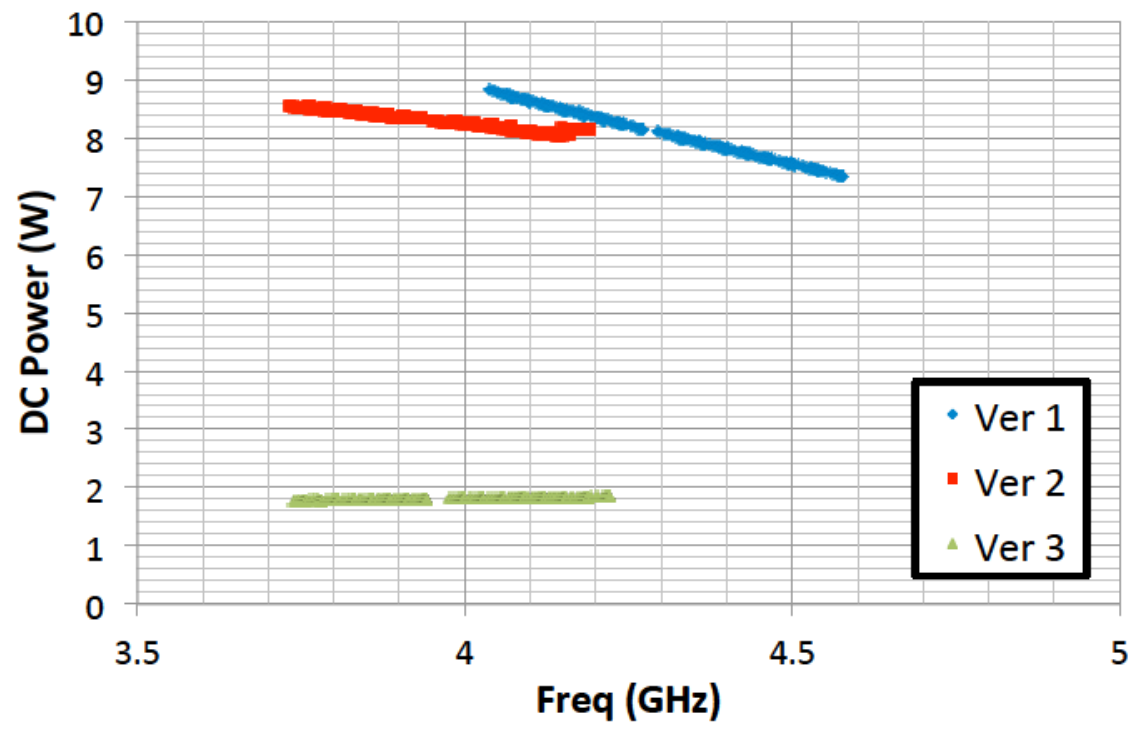

Figure 6.24: Simulated DC power consumption of the three oscillator versions 


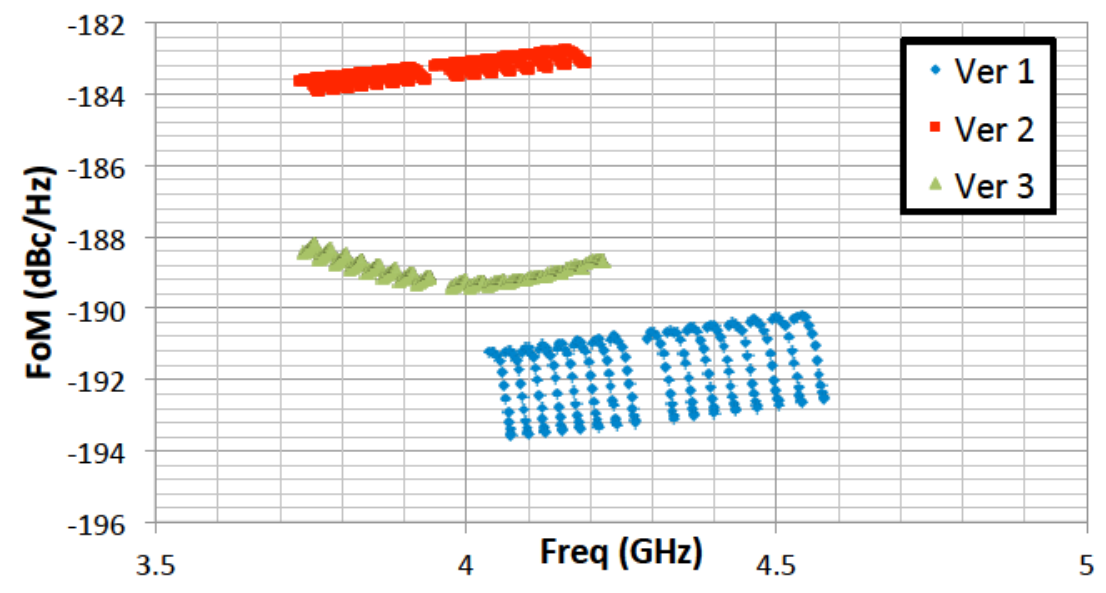

Figure 6.25: Simulated oscillator figure of merit of the three oscillator versions

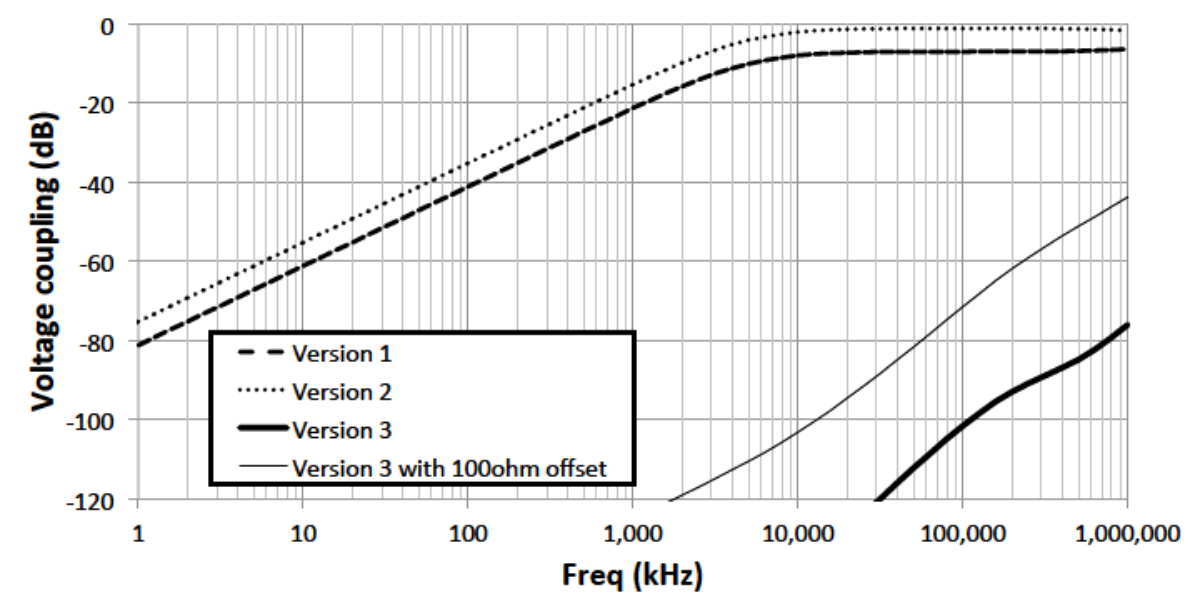

Figure 6.26: Simulated oscillator power supply noise coupling to varactor port for the 3 oscillator versions 
it to be able to compensate for process, voltage, or temperature variations. The design of a calibration circuit to control this diode line is also shown. While parasitic currents place an upper bounds on the switching time of a sub-threshold limited inverter, modulating the control signal allows the rise/fall time to be further extended by gradually changing the duty cycle of a pulse-width modulated control signal. This essentially implements a DAC, but can operate at a low rate without the need for explicit $\mathrm{RC}$ components to act as a reconstruction filter, allowing implementation to be much smaller. Finally, additional methods of reducing the susceptibility of complementary cross-coupled VCOs to power supply noise by significantly attenuating the major path allowing power supply noise to be up-converted to phase noise. The developments further enhance the performance of the circuit principles discussed in the previous chapters. 


\section{Chapter 7}

\section{Conclusion}

\subsection{Summary}

As discussed in Chapter 1, in recent years, communications systems have become increasingly important with the rise of personal mobile devices and the internet of things. With this has come increasing demand for bandwidth in communication systems of all types, requiring greater performance in all of communications systems. Because of diminishing returns in the geometric scaling of CMOS processes and the increasing complexity of communications

systems, the demands on circuit blocks have become more stringent, requiring improvements in many facets of their performance.

Many communications systems rely on frequency synthesizers to perform many tasks, including sampling clocks, clocks for digital circuits, timing and synchronization, modulation and demodulation, etc. These roles are very critical and the performance of the system itself may depend on the performance of the frequency synthesizer itself, specifically its output noise and jitter, although many other properties are important as well, including tuning range, and power consumption. One issue that cannot be overlooked is the ability of the frequency synthesizer to maintain lock over a wide set of conditions. Changes in temperature or supply voltage can cause the desired output frequency to fall out of range of the oscillator inside the frequency synthesizer. While typically the oscillator can compensate for this by having multiple selectable frequency bands, changing bands results in a temporary loss of lock due to a phenomenon called cycle slipping, where the phase-locked loop forming the frequency synthesizer leaves its linear range of operation. When this occurs, the phaselocked loop becomes slow to realign the phase of the oscillator, and multiple phase cycles are "slipped". Thus, even when lock is reacquired, the phase of the reference oscillator locked to is different from what was locked to previously.

In many applications, continuous phase lock is desired or required, while the temporary 
loss of lock can cause issues, the loss of phase lock can cause much larger problems. For instance, when used to clock data, multiple bits may be skipped or scanned twice, resulting in completely erroneous data frames. A detection system which would operate at a higher level of the communications stack would be required to detect such a loss in lock and realign the clocks again, such as by performing a system reset. Many applications have high demands for reliability and uptime in order to achieve high data rates, such as coherent optical transceivers and backplane or wireline transceivers. Loss of phase lock can cause problems in lower power wireless systems such as wireless sensor networks or low power IoT nodes which operate the majority of the time with the communications circuits disabled to lower power. Thus transmitter and receiver pairs must be aligned. If timing alignment tolerances are kept low to reduce power consumption, phase offsets in the synchronization clocks can cause pairs to lose each other, requiring an energy expensive resynchronization handshake.

Thus, it is desirable to prevent these cycle slips and the resulting phase offsets from occurring. In chapter 2 , it was shown that in the literature, there are many different methods presented to prevent this. A common approach used in conventional VCO design is the use of large frequency bands, allowing it to cover a specified frequency range over a wide temperature range. However, the large range required results in a high gain on the oscillator control signal, increasing output phase noise and spurs. This approach also limits the ability to use lower supply voltages for the oscillator, preventing power consumption from decreasing. Another possibility is the stabilization of the oscillator itself to prevent temperature changes from affecting the oscillator's frequency, as is common with crystal reference oscillators. However, this can require off-chip components for the resonator (or expensive wafer processing steps) and is difficult to implement at high frequencies. Tunability is also difficult to implement. A third option is the use of dual-path PLLs, where an additional tuning port is implemented with a high gain and wide tuning range, and is placed in a low frequency loop. The high gain tuning port can increase phase noise, and the loop must be designed carefully to avoid instability. The low frequency loop is also difficult to implement due to its small time constant requiring large passives for implementation. Digital implementation is possible, but is not trivial due to the low noise requirements and the complicated routing required.

Chapter 3 discusses an alternate method. The method shown here differs from previous solutions in that it allows for frequency band changes to take place, only greatly attenuating the resulting transient phase effects so as to prevent cycle slipping and its resulting phase offsets. This is done by slowly switching the capacitor banks implementing the oscillator frequency banks. This allows for gradual frequency band transitions to take place while preventing the phase locked loop from entering non-linear cycle slipping, and thereby limiting 
the maximum phase error to a predetermined amount, and ensuring that the final PLL phase, relative to the reference clock is the same after the frequency band transition as before. The system level concepts and the theoretical circuit behaviour behind this are analyzed and the circuit can be designed to suit the specifications of the system where it is implemented.

This circuit in turn has been verified through the use of a test chip in a low cost process, the design of was discussed in chapter 4 . The use of transient measurement analysis to verify the measurements has also be shown in chapter 5 by showing that the slow switching circuit is able to prevent cycle slipping during frequency band switches that would otherwise occur. The need for this circuit is also demonstrated by operating with varied environmental temperature or supply voltages. The locked behaviour of the PLL has also been shown, showing that low phase noise and jitter (relative to power consumption) can be achieved, even in a low cost, low speed process, with some design compromises made due to limited extraction and verification tool licenses.

Several circuit improvements have been developed and discussed in chapter 6, including adding control circuitry to the slow switching circuit to be able to control the switching time to compensate for variations in process, voltage or temperature. The control circuit can also be modulated with a varying duty cycle pulse-width modulation signal to dither the control line, allowing the transition time to be extended well beyond what would normally be possible. Finally, power supply noise analysis of complementary cross-coupled pair oscillators is done, showing a change in the resonator circuit allows the power supply noise up conversion to the varactor port to be greatly attenuated, further reducing the effect that the power supply noise has on the the oscillator output.

\subsection{Contributions}

Thus, the principal contributions of the proposed work are as follows:

- Analysis of a phase locked loop system, demonstrating that stable operation in its linear region can be maintained with a gradual VCO frequency band transition and analysis of conventional VCO frequency band implementations, showing that gradual frequency band transitions can be made to take place with appropriate switch circuitry and control signal generation

- Design and implementation of an appropriate switching circuit that can be used to create gradual frequency band transitions with conventional VCO frequency band circuits that is compatible with legacy layouts, is easily implementable, is stable and predictable over temperature and process corners, uses minimal silicon area or power 
consumption, and has minimal impact on the PLL during its normal operation. This is in addition to the verification of the complete system in simulation and in measurement, showing transient suppression of $99.3 \%$ and the prevention of cycle slipping

- The modification of the slow switching circuitry to be controllable and stable over process, voltage and temperature with the use of a calibration circuit

- The modification to the control circuitry of the slow switching circuit to allow rise/fall times to be extended arbitrarily

- Analysis of power supply noise up-conversion in complementary cross coupled VCOs and the development of cancellation circuitry using capacitive and magnetic coupling to prevent or reduce this, reducing the phase noise by $12 \mathrm{~dB}$ (as shown in simulation)

\subsection{Future work}

Future work based on the work shown here is possible, and includes incorporating all of the circuit improvements together in a circuit, including the calibration and control circuitry, with an automatic frequency calibration circuit. Improved performance can be obtained with fewer design tradeoffs. Improved measurement quality can be obtained by allowing the VCO control voltage to be directly measured, making frequency vs. time relationships easier to determine. The switching techniques can be incorporated into a DCO, potentially to improve frequency resolution, which could be used to create an ADPLL. Finally, the design can be scaled into different processes to see how it can be used with smaller geometry CMOS processes at high frequencies, and lower supply voltages 


\section{References}

[1] Cisco Systems, "Cisco visual networking index: Forecast and methodology, 2014-2019," Cisco Systems White Paper, Tech. Rep., 2015.

[2] S. K. Korotky, "Semi-empirical description and projections of internet traffic trends using a hyperbolic compound annual growth rate," Bell Labs Tech. J., vol. 18, no. 3, pp. 5-21, Dec. 2013.

[3] L. Atzori, A. Iera, and G. Morabito, "The internet of things: A survey," Computer Networks, vol. 54, no. 15, pp. 2787-2805, Oct. 2010.

[4] J. Maes and C. J. Nuzman, "The past, present and future of copper access," Bell Labs Tech. J., vol. 20, pp. 1-10, 2015.

[5] P. J. Winzer, "Spatial multiplexing in fiber optics: The 10x scaling of metro/core capacities," Bell Labs Tech. J., vol. 19, pp. 22-30, Sept. 2014.

[6] O. N. Foundation, "Software-defined networking: The new norm for networks," April 2012, White paper.

[7] C. R. Stevenson, G. Chouinard, Z. Lei, W. Hu, S. J. Shellhammer, and W. Caldwell, "IEEE 802.22: The first cognitive radio wireless regional area network standard," IEEE Commun. Mag., vol. 47, no. 1, pp. 130-138, 2009.

[8] S. Lee, B. Jagannathan, S. Narasimha, A. Chou, N. Zamdmer, J. Johnson, R. Williams, L. Wagner, J. Kim, J.-O. Plouchart, J. Pekarik, S. Springer, and G. Freeman, "Record RF performance of 45-nm SOI CMOS technology," in Proc. Int. Electron Devices Meeting, Dec. 2007, pp. 255-258.

[9] W. M. Holt, "Moore's law: A path going forward," in IEEE Int. Solid State Circuits Conf. Dig. Tech. Papers, 2016, pp. 8-13. 
[10] T. Ohguro, Y. Higashi, K. Okano, S. Inaba, and Y. Toyoshima, "The optimimum device parameters for high RF and analog/MS performance in planar MOSFET and FinFET," in Symp. VLSI Dig. Tech. Papers, 2012, pp. 149-150.

[11] T. Ohguro, S. Inaba, A. Kaneko, and K. Okano, "Technology of FinFET for high RF and analog/mixed-signal performance circuits," IEICE Trans. Electron., vol. E98, no. 6 , pp. 455-460, Jun. 2015.

[12] P. Kinget, "Scaling analog circuits into deep nanoscale CMOS: Obstacles and ways to overcome them," in Proc. IEEE Custom Integrated Circuits Conf., Sept. 2015, pp. 1-8.

[13] F. Schwierz, "Graphene transistors: Status, prospects and problems," Proc. IEEE, vol. 101, no. 7, pp. 1567-1584, Jul. 2013.

[14] P. Zheng, D. Connelly, F. Ding, and T.-J. Liu, "FinFET evolution toward stackednanowire FET for CMOS technology scaling," IEEE Trans. Elec. Devices, vol. 62, no. 12, pp. 3945-3950, Dec. 2015.

[15] R. Kim, U. E. Avci, and I. A. Young, "CMOS performance benchmarking of Si, InAs, GaAs, and Ge nanowire n- and pMOSFETs with $\mathrm{LG}=13 \mathrm{~nm}$ based on atomistic quantum transport simulation including strain effects," in IEEE Int. Elec. Dev. Meeting Tech. Dig. Papers, Dec. 2015.

[16] W. Y. Choi, B.-G. Park, J. D. Lee, and T.-J. K. Liu, "Tunneling field-effect transistors (TFETs) with subthreshold swing (SS) less than $60 \mathrm{mV} / \mathrm{dec}$," IEEE Elec. Dev. Letters, vol. 28 , no. 8 , pp. 743-745, Aug. 2007.

[17] S. S. P. Parkin, "Spintronic materials and devices: past, present and future!" in IEEE Int. Elec. Dev. Meeting Tech. Dig. Papers, Dec. 2004, pp. 903-906.

[18] S. A. Wolf, J. Lu, M. R. Stan, E. Chen, and D. M. Treger, "The promise of nanomagnetics and spintronics for future logic and universal memory," Proc. IEEE, vol. 98, no. 98, pp. 2155-2168, Dec. 2010.

[19] World Semiconductor Trade Statistics, "WSTS semiconductor market forecasts 20032016," accessed Jun 6,2016. [Online]. Available: http://www.wsts.org/PRESS/RecentNews-Release

[20] W. Sansen, "Analog CMOS from 5 micrometer to 5 nanometer," in IEEE Int. SolidState Circuits Conf. Dig. Tech. Papers, Feb. 2015, pp. 1-8. 
[21] D. Griffith, "Calibration techniques for reference oscillators used in IoT," in IEEE RFIC Symp. Workshop, May 2016.

[22] X. Gao, E. A. M. Klumperink, M. Bohsali, and B. Nauta, "A $2.2 \mathrm{GHz} 7.6 \mathrm{~mW}$ subsampling PLL with $-126 \mathrm{dBc} / \mathrm{Hz}$ in-band phase noise and 0.15 psrms jitter in $0.18 \mathrm{um}$ CMOS," in IEEE ISSCC Dig, San Francisco, 2009, pp. 392-394.

[23] X. Gao, E. Klumperink, and B. Nauta, "Sub-sampling PLL techniques," in Proc. IEEE Custom Integrated Circuits Conf., Sept. 2015.

[24] S. Levantino, D. Tasca, M. Giovanni, M. Zanuso, C. Samori, and A. L. Lacita, "A wideband fractional-N PLL with suppressed charge-pump noise and automatic loop filter calibration," in IEEE RFIC Symp Dig., Montreal, 2012, pp. 177-180.

[25] W. C. Lindsey and C. M. Chie, "A survey of digital phase-locked loops," Proc. IEEE, vol. 69, no. 4, pp. 410-431, April 1981.

[26] G. Pasternack and R. L. Whalin, "Analysis and synthesis of a digital phase-locked loop for FM demodulation," Bell Sys. Tech. J., vol. 47, no. 10, pp. 2207-2237, 1968.

[27] R. Saban and A. Efendovich, "A fully-digital, 2-MB/sec, CMOS data separator," in Proc. IEEE Int. Symp. Circuits and Sys., Jun. 1994, pp. 53-56.

[28] S. Fukuda, "A new digital PLL for the class I partial response channel," IEEE Trans. Magnetics, vol. 32, no. 5, pp. 3974-3976, Sept. 1996.

[29] T.-Y. Hsu, B.-J. Shieh, and C.-Y. Lee, "An all-digital phase-locked loop (ADPLL)based clock recovery circuit," IEEE. J. Solid-State Circuits, vol. 34, no. 8, pp. 10631073, Aug 1999.

[30] T. Watanbe and S. Yamauchi, "An all-digital PLL for frequency multiplication by 4 to 1022 with seven-cycle lock time," IEEE. J. Solid-State Circuits, vol. 38, no. 2, pp. 198-204, Feb. 2003.

[31] C.-C. Chung and C.-Y. Lee, "An all-digital phase-locked loop for high-speed clock generation," IEEE. J. Solid-State Circuits, vol. 38, no. 2, pp. 347-351, Feb 2003.

[32] R. Fried and Z. Azmanov, "Low-power digital PLL with one cycle frequency lock-in time and large frequency-multiplication factor for advanced power management," in Proc. IEEE Int. Conf. Elec. Circuits and Systems, Oct. 1996, pp. 1166-1169. 
[33] I. Hwang, S. Lee, S. Lee, and S. Kim, "A digitally controlled phase-locked loop with fast locking scheme for clock synthesis application," in IEEE Int. Solid-State Circuits Conf. Dig. of Tech. Papers, Feb. 2000, pp. 168-169.

[34] J. H.-C. Lin, B. Haroun, T. Foo, J.-S. Wang, B. Helmick, S. Randall, T. Mayhugh, C. Barr, and J. Kirkpatrick, "A PVT tolerant 0.18-MHz to $600-\mathrm{MHz}$ self-calibrated digital PLL in 90-nm CMOS process," in IEEE Int. Solid-State Circuits Conf. Dig. of Tech. Papers, Feb. 2004, pp. 488-490.

[35] R. B. Staszewski, J. L. Wallberg, S. Rezeq, C.-M. Hung, O. E. Eliezer, S. K. Vemulapalli, C. Fernando, K. Maggio, R. Staszewski, N. Barton, M.-C. Lee, P. Cruise, M. Entezari, K. Muhammad, and D. Leipold, "All-digital PLL and transmitter for mobile phones," Journal of Solid-State Circuits, vol. 40, no. 12, pp. 2469-2482, 2005.

[36] D. C. Wei, Y. Huang, B. W. Garlepp, and J. Hein, "A monolithic low-bandwidth jittercleaning PLL with hitless switching for SONET/SDH clock generation," in IEEE Int. Solid-State Circuits Conf. Dig. of Tech. Papers, Feb 2006, pp. 884-893.

[37] C.-M. Hsu, M. Z. Straayer, and M. H. Perrott, "A low-noise wide-BW 3.6-GHz digital $\Delta \Sigma$ fractional-N frequency synthesizer with a noise-shaping time-to-digital converter and quantization noise cancellation," IEEE. J. Solid-State Circuits, vol. 43, no. 12, pp. 2776-2786, Dec. 2008.

[38] C.-W. Yao and A. N. W. Jr., "A 2.8-3.2-GHz fractional-N digital PLL with ADCassisted TDC and inductively coupled fine-tuning DCO," IEEE. J. Solid-State Circuits, vol. 48, no. 3, pp. 698-710, Mar. 2013.

[39] C. Venerus and I. Galton, "A TDC-free mostly-digital FDC-PLL frequency synthesizer with a 2.8-3.5 GHz DCO," IEEE. J. Solid-State Circuits, vol. 50, pp. 450-463, Feb. 2015.

[40] P. Dudek, S. Szczepański, and J. . Hatfield, "A high-resolution CMOS time-to-digital converter utilizing a Vernier Delay Line," IEEE Trans. Solid-State Circuits, vol. 35, no. 2, pp. 240-247, Feb. 2000.

[41] V. Ramakrishnan and P. T. Balsara, "A wide-range, high-resolution, compact, CMOS time to digital converter," in Proc. Int. Conf. VLSO Design, Jan 2006.

[42] L. Vercesi, A. Liscidini, and R. Castello, "Two-dimensions Vernier time-to-digital converter," IEEE. J. Solid-State Circuits, vol. 45, no. 8, pp. 1504-1512, Aug. 2010. 
[43] J.-P. Jansson, A. Mäntyniemi, and J. Kostamovaara, "A CMOS time-to-digital converter with better than 10-ps single-shot precision," IEEE. J. Solid-State Circuits, vol. 41, no. 6, pp. 1286-1296, Jun. 2006.

[44] S. Henzler, D. Lorenz, W. Kamp, R. Kuenemund, and D. Schmitt-Landsiedel, "Variation tolerant high resolution and low latency time-to-digital converter," in Proc. European Solid-State Circuits Conf., Sept. 2007, pp. 194-197.

[45] M. Z. Straayer and M. H. Perrott, "A multi-path gated ring oscillator TDC with firstorder noise shaping," IEEE. J. Solid-State Circuits, vol. 44, no. 4, pp. 1089-1098, April 2009.

[46] Y. Cao, W. D. Cock, M. Steyaert, and P. Leroux, "1-1-1 MASH $\Delta \Sigma$ time-to-digital converters with 6-ps resolution and third-order noise-shaping," IEEE. J. Solid-State Circuits, vol. 47, no. 9, pp. 2093-2106, Sept 2012.

[47] J. Yu, F. F. Dai, and R. C. Jaeger, "A 12-bit Vernier ring time-to-digital converter in

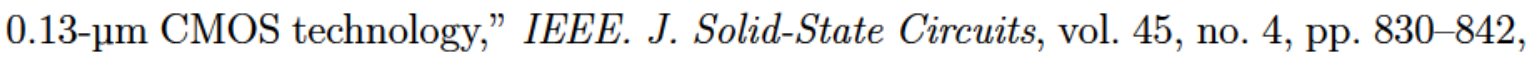
April 2010.

[48] P. Chen, S.-I. Liu, and J. Wu, "A CMOS pulse-shrinking delay element for time interval measurement," IEEE Trans. Circuits and Sys. II, vol. 47, no. 9, pp. 954-958, Sept. 2000.

[49] M. Lee and A. A. Abidi, "A 9-b, 1.25-ps resolution coarse-fine time-to-digital converter in 90-nm CMOS that amplifies a time residue," IEEE. J. Solid-State Circuits, vol. 43, no. 4, pp. 769-777, Apr. 2008.

[50] Y.-H. Seo, J.-S. Kim, H.-J. Park, and J.-Y. Sim, "A 0.63-ps resolution, 11-b pipeline

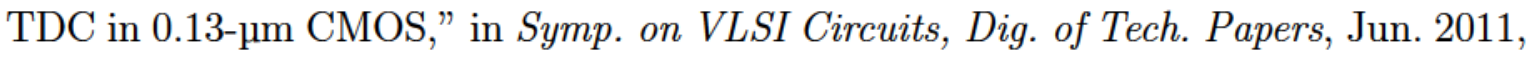
pp. $152-153$.

[51] L. Fanori, A. Liscidini, and R. Castello, "Capacitive degeneration in LC-tank oscillator for DCO fine-frequency tuning," IEEE J. of Solid-State Circuits, vol. 45, no. 12, pp. 2737-2745, Dec. 2010.

[52] L. Vercesi, L. Fanori, F. D. Bernardinis, A. Liscidini, and R. Castello, "A dither-less all digital PLL for cellular transmitters," IEEE J. of Solid-State Circuits, vol. 47, no. 8, pp. 1908-1920, 2012. 
[53] W. Wu, X. Bai, R. B. Staszewski, and J. R. Long, "A 56.4-to-63.4 GHz spurious-free all-digital fractional-N PLL in $65 \mathrm{~nm}$ CMOS," in IEEE Int. Solid-State Circuits Conf. Dig. of Tech. Papers, Feb. 2013, pp. 352-353.

[54] R. E. Best, Phase-Locked Loops: Theory, Design and Applications. Mcgraw-Hill, 1993.

[55] B. Razavi, Monolithic phase-locked loops and clock recovery circuits: Theory and design. Wiley-IEEE Press, 1996.

[56] J. W. M. Rogers, C. Plett, and F. Dai, Integrated Circuit Design for High-Speed Frequency Synthesis. Artech House, 2006.

[57] S. R. Karri, B. S. Heng, C. H. Shen, and G. K. Dehng, "Temperature compensated VCO design for multi-band WCDMA transceiver application," in Proc. IEEE Int. Symp. Radio-Frequency Integration Technology, 2009, pp. 191-194.

[58] V. C. de Beaupré, W. Rahajandraibe, L. Zaïd, and G. Bas, "A CMOS 2.45-GHz ring oscillator with temperature compensation," in Elec., Circuits and Systems, IEEE Int. Conf. on, Dec 2005, pp. 1-4.

[59] M. S. McCorquodale, J. D. O'Day, S. M. Pernia, G. A. Carichner, S. Kubba, and R. B. Brown, "A monolithic and self-referenced RF LC clock generator compliant with USB 2.0," IEEE. J. Solid-State Circuits, vol. 42, no. 2, pp. 385-399, 2007.

[60] P. C. Maulik and P. W. Lai, "frequency tuning of wide temperature range CMOS LC VCOs," IEEE J. of Solid-State Circuits, vol. 46, no. 9, pp. 2033-2040, Sept. 2011.

[61] Y. Ueno and H. Shimizu, "Voltage controlled temperature compensated crystal oscillator using 2-port crystal resonator," in Proc. of the IEEE Frequency Control Symp., May 1991, pp. 418-425.

[62] M. P. Cracknell, A. M. Harrison, and D. J. Sharpe, "A new technique for the temperature compensation of SAW oscillators," in Freq. Control and Synthesis, Int. Conf. on, Apr. 1989, pp. 49-52.

[63] M. A. Taslakov, "A temperature compensated $1 \mathrm{GHz}$ STW based multifrequency oscillator," in Proc. of the IEEE Radio Freq. Integrated Circuits Symp., Jun. 1993, pp. $740-743$.

[64] C. Zuo, J. V. der Spiegel, and G. Piazza, "1.5-GHz CMOS voltage-controlled oscillator based on thickness-field-excited piezoelectric AlN contour-mode MEMS resonators," in Proc. IEEE Custom Integrated Circuits Conf., Sept 2010, pp. 1-4. 
[65] S. Razafimandimby, D. Petit, P. Bar, S. Joblot, J.-F. Carpentier, J. Morelle, C. Arnaud, G. Parat, P. Garcia, and C. Garnier, "Co-design considerations for frequency drift compensation in BAW-based time reference applications," in Proc. IEEE RFIC Symp., May 2010, pp. 249-252.

[66] W.-T. Hsu, J. R. Clark, and C. T.-C. Nguyen, "A sub-micron capacitive gap process for multiple-metal-electrode lateral micromechanical resonators," in Proc. IEEE Int. Conf. MEMS, Jan. 2001, pp. 349-352.

[67] Y.-W. Lin, S. Lee, Z. Ren, and C. T. C. Nguyen, "Series-resonant micromechanical resonant oscillator," in IEEE Int. Electron Devices Meeting Tech. Dig., Dec. 2003, p. 39.4.1 39.4.4.

[68] B. P. Otis and J. M. Rabaey, "A 300- $\mu \mathrm{W} 1.9-\mathrm{GHz}$ CMOS oscillator utilizing micromachined resonators," IEEE. J. Solid-State Circuits, vol. 38, no. 7, pp. 1271-1274, Jul. 2003.

[69] Y.-W. Lin, S. Lee, S.-S. Li, Y. Xie, Z. Ren, and C. T.-C. Nguyen, "60-MHz wine-glass micromechanical-disk reference oscillator," in IEEE Int. Solid-State Circuits Conf. Dig. of Tech. Papers, Feb. 2004.

[70] E. P. Quevy and R. T. Howe, "Redundant MEMS resonators for precise reference oscillators," in IEEE RFIC Symp Dig., Jun. 2005, pp. 113-116.

[71] C. S. Lam, "A review of the recent development of MEMS and crystal oscillators and their impact on the frequency control products industry," in Proc. Ultrasonics Symp., Nov 2008, pp. 694-704.

[72] C.-S. Li, M.-H. Li, C.-H. Chin, C.-Y. Chen, S.-S. Li, and P. X.-L. Feng, "A piezoresistive CMOS-MEMS resonator with high Q and low TCf," in European Frequency and Time Forum and Int. Frequency Control Symp Proc., Jul. 2013, pp. 425-428.

[73] S.-S. Li, "CMOS-MEMS resonators and their applications," in European Frequency and Time Forum and Int. Frequency Control Symp Proc., Jul. 2013, pp. 915-921.

[74] G. Chance, T. Meyer, S. Stoeckl, B. Neurauter, G. Patane, G. Minichshofer, J. H. Kuypers, J. Schoepf, R. Revel, D. Weninger, K. Chung, T. S. Chew, and O. Mendoza, "Integrated MEMS oscillator for cellular transceivers," in Proc. IEEE Int. Frequency Control Symp., May 2014, pp. 1-3. 
[75] S. M. Kashmiri, M. A. P. Pertijs, and K. A. A. Makinwa, "A thermal-diffusion-based frequency reference in standard CMOS with an absolute inaccuracy of $\pm 0.1 \%$ From $-55^{\circ} \mathrm{C}$ to $125^{\circ} \mathrm{C}$," IEEE. J. Solid-State Circuits, vol. 45 , no. 12, pp. 2510-2520, Dec. 2010.

[76] M. Schallner and W. Konrath, "Adjustment of a temperature compensated Ka-band ring resonator VCO using fully automated laser-trimming," in IEEE MTT-S Int. Microwave Symp. Dig., vol. 3, May 2001, pp. 2179-2182.

[77] A. Dieguez, A. Arbat, A. Sanuy, R. Casanova, and M. R. J. Samitier, "A wake-up circuit with temperature compensated clock in 1.2V-0.13pm CMOS technology," in Elec. Circuits and Systems, IEEE Int. Conf on, Dec. 2007, pp. 367-370.

[78] X. Zhang and A. B. Apsel, "A low-power, process-and-temperature-compensated ring oscillator with addition-based current source," IEEE Trans. Circuits Syst. I, Reg. Papers, vol. 58, no. 5, pp. 868-878, May 2011.

[79] J. Nebhen, S. Meillère, and M. Masmoudi, "A temperature compensated CMOS ring oscillator for wireless sensing appications," in Proc. IEEE New Circuits and Systems Conf., June 2012, pp. 37-40.

[80] T. Sowlati and H. Shabkiba, "A 20-800 MHz relaxion oscillator with automatic swing control," in IEEE Int. Solid-State Circuits Conf. Dig. of Tech. Papers, Feb 1998, pp. $222-223$.

[81] Y. Sun, H. G. van Veenendaal, and J. L. Tauritz, "Temperature analysis and on-chip compensation for an UHF VCO," in Microwave Symp. Dig., IEEE MTT-S Int., June 1997, pp. 757-760.

[82] Z.-M. Lin, K.-C. Huang, J.-D. Chen, and M.-Y. Liao, "A CMOS voltage-controlled oscillator with temperature compensation," in Proc. of Asia Pacific Conf. on ASICs, Aug 2000, pp. 85-86.

[83] A. P. Behera, S. Sasmal, and P. Nandi, "A wide range CMOS VCO for PLL applications," in Proc. Int. Conf. VLSI Design, 2013, pp. 163-168.

[84] S.-J. Park, S. Woo, H. Ha, Y. Suh, H.-J. Park, and J.-Y. Sim, "A transistor-based background self-calibration for reducing PVT sensitivity with a design example of an adaptive bandwidth PLL," in IEEE Asian Solid-State Circuits Conference, Nov. 2008, pp. $433-436$. 
[85] J. C. Salvia, R. Melamud, S. A. Chandorkar, S. F. Lord, and T. W. Kenny, "Real-time temperature compensation of MEMS oscillators using an integrated micro-oven and a phase-locked loop," J. Microelectromechanical Systems, vol. 19, no. 1, pp. 192-201, Feb. 2010.

[86] Y.-S. Shyu and J.-C. Wu, "A process and temperature compensated ring oscillator," in ASICs, IEEE Asia Pacific Conf. on, Aug. 1999, pp. 283-286.

[87] W.-H. Chan, J. Lau, and A. Buchwald, "A 622-MHz interpolating ring VCO with temperature compensation and jitter analysis," in Circuits and Systems, IEEE Int. Symp. on, Jun. 1997, pp. 25-28.

[88] K. Stadius, J. Holmberg, R. Kaunisto, P. Alinikula, and V. Porra, "A monolithic temperature compensated 1.6 GHz VCO," in Proc. Eur. Microwave Conf., Oct 1998, pp. 217-221.

[89] S.-S. Lee, T.-G. Yoo, and S.-W. Kim, "Process-and-temperature compensated CMOS oscillator for clock generators," Electronics Letters, vol. 39, no. 21, pp. 1484-1485, Oct. 2003.

[90] K. Sundaresan, K. C. Brouse, K. U-Yen, F. Ayazi, and P. E. Allen, "A 7-MHz process, temperature and supply compensated clock oscillator in $0.25 \mu \mathrm{m}$ CMOS," in Circuits and Systems, IEEE Int. Symp. on, May 2003, pp. 693-696.

[91] J. Kim, M. A. Horowitz, and G.-Y. Wei, "Design of CMOS adaptive-bandwidth PLL/DLLs: A general approach," IEEE Trans. Circuits Syst. II: Analog and Digital Signal Processing, vol. 50, no. 11, pp. 860-869, Nov. 2003.

[92] J. Midtgaard, T. Jeppesen, K. T. Christensen, E. Bruun, and P. Andreani, "Fully integrated $1.7 \mathrm{GHz}, 188 \mathrm{dBc} / \mathrm{Hz}$ FoM, $0.8 \mathrm{~V}, 320 \mu \mathrm{W}$ LC-tank VCO and frequency divider," in Symp. on VLSI Circuits, Dig. of Tech. Papers, Jun. 2005, pp. 244-247.

[93] K. Sundaresan, P. E. Allen, and F. Ayazi, "Process and temperature compensation in a 7-MHz CMOS clock oscillator," IEEE J. of Solid-State Circuits, vol. 41, no. 2, pp. 433-442, Feb. 2006.

[94] K. R. Lakshmikumar, V. Mukundagiri, and S. L. J. Gierkink, "A process and temperature compensated two-stage ring oscillator," in Proc. of the IEEE Custom Integrated Circuits Conference, Sept. 2007, pp. 691-694. 
[95] H. Chen, S. Li, Q. Niu, Y. Wu, and F. Zhou, "A multi-phase self-sensing clock generator for hybrid DPWM application," in Proc. Int. Conf. ASIC, Oct. 2007, pp. 635-638.

[96] Y.-T. Liao and C.-J. R. Shi, "A 6-11 GHz multi-phase VCO design with active inductors," in Circuits and Systems, IEEE Int. Symp.. on, May 2008, pp. 988-991.

[97] R. Vijayaraghavan, M. R. Haider, S. K. Islam, and C. Su, "A wideband injection locked frequency divider based on a process and temperature compensated ring oscillator," in Proc. IEEE Radio and Wireless Symp., Jan. 2008, pp. 379-382.

[98] R. Vijayaraghavan, S. K. Islam, M. R. Haider, and L. Zuo, "Wideband injection-locked frequency divider based on a process and temperature compensated ring oscillator," Circuits, Device, E3 Systems, IET, vol. 3, no. 5, pp. 259-267, Oct. 2009.

[99] T. Nakamura, T. Masuda, K. ichi Ohhata, K. Watanabe, H. Yoshioka, T. Kusunoki, M. Tanabe, A. Koyama, T. Harada, and K. Washio, "A fully integrated 39.8-/43$\mathrm{GHz} \mathrm{VCO}$ - featuring wide tuning range and low temperature drift-for single-chip MUX/DEMUX LSIs," in Proc. of the IEEE Radio Freq. Integrated Circuits Symp., Jun. 2004, pp. 317-320.

[100] S. Kishimoto, K. Maruhashi, M. Ito, T. Morimoto, Y. Hamada, and K. Ohata, "A 60GHz-band subharmonically injection locked VCO MMIC operating over wide temperature range," in Microwave Symp. Dig., IEEE MTT-S Int., Jun. 2005, pp. 1689-1692.

[101] Y. Fukasawa, K. Kawaguchi, T. Yoshida, T. Sugiyama, and A. Nakagawa, "76-GHz planar Gunn VCO with low oscillation-frequency drift of $1.5 \mathrm{MHz} /{ }^{\circ} \mathrm{C}$," in Proc. of Asia-Pacific Microwave Conf., Dec. 2007, pp. 1-4.

[102] C.-Y. Yu, J.-Y. Yu, and C.-Y. Lee, "An eCrystal oscillator with self-calibration ability," in Circuits and Systems, IEEE Int. Symp. on, May 2009, pp. 24-27.

[103] K. AbuGharbieh, M. Abdelfattah, T. Al-Maaita, and A. Tahboub, "A wide tuning range $11.8 \mathrm{GHz}$ ring oscillator $\mathrm{VCO}$ with temperature and process compensation," in EUROCON, 2013 IEEE, Jul. 2013, pp. 1844-1848.

[104] Y.-C. Huang, C.-F. Liang, H.-S. Huang, and P.-Y. Wang, "A 2.5 GHz ADPLL with digital-regulated supply-noise-insensitive and temperature-self-compensated ring DCO," in IEEE Int. Solid-State Circuits Conf. Dig. of Tech. Papers, Feb. 2014, pp. $270-271$. 
[105] T. Tanzawa, H. Shibayama, R. Terauchi, K. Hisano, H. Ishikuro, S. Kousai, H. Kobayashi, H. Majima, T. Takayama, K. Agawa, M. Koizumi, and F. Hatori, "A temperature-compensated CMOS LC-VCO enabling the direct modulation architecture in $2.5 \mathrm{GHz}$ GFSK transmitter," in Proc. of the IEEE Custom Integrated Circuits Conference, 2004, pp. 273-276.

[106] R. Geisler, J. Liobe, and M. Margala, "Process and temperature calibration of PLLs with BiST capabilities," in Circuits and Systems, IEEE Int. Symp.. on, May 2007, pp. 3864-3867.

[107] V. Melikyan, A. Balabanyan, A. Hayrapetyan, and A. Durgaryan, "NMOS/PMOS resistance calibration method using reference frequency," in Proc. Computer Science and Information Technologies, Sept. 2013, pp. 1-6.

[108] D. L. Buck and L. E. Hoff, "Digital design for a self-temperature compensating oscillator," in Proc. IEEE Int. Frequency Control Symp., 2002, pp. 604-609.

[109] T. Kobayashi, H. Iwamoto, and T. Hara, "A digitally temperature compensated compact PLL module," in Proc. IEEE Int. Frequency Control Symp., May 1997, pp. 969974 .

[110] J. Wang and W. L. Goh, "A 13.5-MHz relaxation oscillator with $\pm 0.5 \%$ temperature stability for RFID application," in Proc. IEEE NEWCAS, 2016, pp. 2431-2434.

[111] M. Choi, T. Jang, S. Band, Y. Shi, D. Blaauw, and D. Sylvester, "A $110 \mathrm{nW}$ resistive frequency locked on-chip oscillator with $34.3 \mathrm{ppm} /{ }^{\circ} \mathrm{c}$ temperature stability for systemon-chip designs," IEEE J. Solid State Circuits, Jul. 2016.

[112] B. Saeidi, J. Cho, G. Taskov, and A. Paff, "A wide-range VCO with optimum temperature adaptive tuning," in Proc. of the IEEE Radio Freq. Integrated Circuits Symp., May 2010, pp. 337-340.

[113] G. K. Montress, T. E. Parker, D. W. Kress, and J. A. Kosinski, "Design and performance of a low noise, wide tuning range AQP SAW delay line VCO," in Proc. of the IEEE Frequency Control Symp., May 1992, pp. 356-370.

[114] T. Sun, C. Hui, and Y. Wang, "A VCO with high supply noise rejection and its application to PLL frequency synthesizer," in Proc. IEEE Int. Symp. Communications and Information Tech., Oct 2005, pp. 1027-1030. 
[115] K. Manetakis, D. Jessie, and C. Narathong, "A CMOS VCO with $48 \%$ tuning range for modern broadband systems," in Proc. of the IEEE Custom Integrated Circuits Conference, Oct. 2004, pp. 265-268.

[116] H. Akima, A. Dec, T. Merkin, and K. Suyama, "A 10 GHz frequency-drift temperature compensated LC VCO with fast-settling low-noise voltage regulator in $0.13 \mu \mathrm{m}$ CMOS," in Proc. of the IEEE Custom Integrated Circuits Conference, 2010, pp. 1-4.

[117] V. Ravinuthula and S. Finocchiaro, "A low power high performance PLL with temperature compensated VCO," in Proc. of the IEEE Radio Freq. Integrated Circuits Symp., 2016, pp. 31-34.

[118] H. G. Ozsema, T. Demirci, and Y. Leblebici, "3.6 GHz CMOS ring oscillator with low tune voltage sensitivity and temperature compensation," in Proc. Nordic Circuits and Systems Conf.: NORCHIP Int. Symp. on System-on-Chip, 2015.

[119] Y. You, D. Huang, J. Chen, and S. Chakraborty, "A 12 GHz $67 \%$ tuning range 0.37 ps $R J_{r m s}$ PLL with LC-VCO temperature compensation scheme in $0.13 \mu \mathrm{m}$ CMOS," in Proc. of the IEEE Radio Freq. Integrated Circuits Symp., June 2014, pp. 101-104.

[120] K.-F. Chong, L. Siek, and B. Lau, "A PLL with a VCO of improved PVT tolerance," in Int. Symp. on Integrated Circuits, Dec. 2011, pp. 464-467.

[121] J. Craninckx and M. S. J. Steyaert, "A Fully Integrated CMOS DCS-1800 Frequency Synthesizer," IEEE J. of Solid-State Circuits, vol. 33, no. 12, pp. 2054-2065, Dec. 1998.

[122] Y. Koo, H. Huh, Y. Cho, J. Lee, J. Park, K. Lee, D.-K. Jeong, and W. Kim, "A fully integrated CMOS frequency synthesizer with charge-averaging charge pump and dualpath loop filter for PCS- and cellular-CDMA wireless systems," IEEE. J. Solid-State Circuits, vol. 37, no. 5, pp. 536-542, May 2002.

[123] B. D. Muer and M. S. J. Steyaert, "A CMOS monolithic $\Delta \Sigma$-controlled fractional-N frequency synthesizer for DCS-1800," IEEE. J. Solid-State Circuits, vol. 37, no. 7, pp. 835-844, Jul. 2002.

[124] J. Yan, H. Zheng, X. Zeng, and T. Tang, "Compact current-mode loop filter for PLL applications," Electronics Letters, vol. 41, no. 23, pp. 1257-1258, Nov. 2005.

[125] S.-J. Li, H.-H. Hsieh, and L.-H. Lu, "A 10 GHz phase-locked loop with a compact lowpass filter in 0.18 um CMOS," IEEE Microwave and Wireless Components Letters, vol. 19, no. 10, pp. 659-661, Sept. 2009. 
[126] X. Meng, L. Huang, L. Chen, and F. Lin, "Area efficient PLL design using capacitance multiplication based on self-biased architecture," in Proc. IEEE Int. Symp. RadioFrequency Integration Tech., 2011, pp. 193-196.

[127] F. Herzel, S. Glisic, S. A. Osmany, J. C. Scheytt, K. Schmalz, W. Winkler, and M. Engels, "A fully integrated 48-GHz low-noise PLL with a constant loop bandwidth," in Silicon Monolithic Integrated Circuits in RF Systems, IEEE Topical Meeting on, Jan 2008, pp. 82-85.

[128] A. Arakali, S. Gondi, and P. K. Hanumolu, "Low-power supply-regulation techniques for ring oscillators in phase-locked loops using a split-tuned architecture," IEEE. J. Solid-State Circuits, vol. 44, no. 8, pp. 2169-2181, Aug. 2009.

[129] S. Levantino, G. Marzin, C. Samori, and A. L. Lacaita, "A wideband fractional-N PLL with suppressed charge-pump noise and automatic loop filter calibration," IEEE. J. Solid-State Circuits, vol. 48, no. 10, pp. 2419-2429, Oct. 2013.

[130] A. L. S. Loke, R. K. Barnes, T. T. Wee, M. M. Oshima, C. E. Moore, R. R. Kennedy, and M. J. Gilsdorf, "A versatile 90-nm CMOS charge-pump PLL for SerDes transmitter clocking," IEEE. J. Solid-State Circuits, vol. 41, no. 8, pp. 1894-1907, Aug. 2006.

[131] H. Chen, E. Lee, and R. Geiger, "A $2 \mathrm{GHz}$ VCO with process and temperature compensation," in Circuits and Systems, IEEE Int. Symp. on, Jul. 1999, pp. 569-572.

[132] S. Kudszus, T. Berceli, A. Tessmann, M. Neumann, and W. H. Haydl, "W-Band HEMT-oscillator MMICs using subharmonic injection locking," Transaction on Microwave Theory and Techniques, vol. 48, no. 12, pp. 2526-2532, 2000.

[133] X. Zhang and A. B. Apsel, "A process compensated 3-GHz ring oscillator," in Circuits and Systems, IEEE Int. Symp.. on, May 2009, pp. 581-584.

[134] Y. Chang, D. Rozenblit, B. Saeidi, J. Leete, M. Kahrizi, J. Chiu, S. Zhou, M. Vadipour, J. Juan, and R. Magoon, "A temperature compensated VCO using feed-forward gain multiplication for cellular applications," in Proc. of the IEEE Radio Freq. Integrated Circuits Symp., 2015, pp. 187-190.

[135] F. Herzel, G. Fischer, and H. Gustat, "An integrated CMOS RF synthesizer for 802.11a wireless LAN," IEEE J. of Solid State Circuits, vol. 38, no. 10, pp. 1767-70, 2001.

[136] W. Winkler, J. Borngraber, B. Heinemann, and F. Herzel, "A Fully Integrated BiCMOS PLL for $60 \mathrm{GHz}$ Wireless Applications," in IEEE ISSCC Dig., 2005, pp. 406-7. 
[137] J. G. Kenney, Declan, Dalton, E. Evans, M. H. Eskiyerli, B. Hilton, D. Hitchcox, T. Kwok, D. Mulcahy, C. McQuilkin, V. Reddy, S. Selvanayagam, P. Shepherd, W. S.

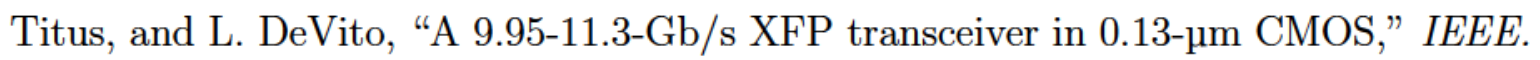
J. Solid-State Circuits, vol. 41, no. 12, pp. 2901-2910, Dec. 2006.

[138] W. Rhee, H. Ainspan, D. J. Friedman, T. Rasmus, S. Garvin, and C. Cranford, "A uniform bandwidth PLL using a continuously tunable single-input dual-path LC VCO for $5 \mathrm{~Gb} / \mathrm{s}$ PCI express Gen2 application," in Proc. IEEE Asian Solid-States Circuits Conf., Nov. 2007, pp. 63-66.

[139] T. Wu, P. K. Hanumolu, K. Mayaram, and U.-K. Moon, "A 4.2 GHz PLL frequency synthesizer with an adaptively tuned coarse loop," in Proc. IEEE CICC, Sept 2007, pp. $547-550$.

[140] D. M. Fischette, A. L. S. Loke, M. M. Oshima, B. A. Doyle, R. Bakalski, R. J. DeSantis, A. Thiruvengadam, C. L. Wang, G. R. Talbot, and E. S. Fang, "A 45nm SOI-CMOS dual-PLL processor clock system for multi-protocol I/O," in IEEE Int. Solid-State Circuits Conf. Dig. of Tech. Papers, Feb. 2010, pp. 246-247.

[141] S. Williams, H. Thompson, M. Hufford, and E. Naviasky, "An improved CMOS ring oscillator PLL with less than 4ps RMS accumulated jitter," in Proc. IEEE Custom Integrated Circuits Conf., Oct. 2004, pp. 151-154.

[142] R. Nonis, N. D. Dalt, P. Palestri, and L. Selmi, "Modelling, design and characterization of a new low-jitter analog dual tuning LC-VCO PLL architecture," IEEE. J. SolidState Circuits, vol. 40, no. 6, pp. 1303-1309, Jun. 2005.

[143] R. He, C. Liu, X. Yu, W. Rhee, J.-Y. Park, C. Kim, and Z. Wang, "A low-cost, leakage-insensitive semi-digital PLL with linear phase detection and FIR-embedded digital frequency acquisition," in Proc. IEEE Asian Solid-State Circuits Conf., Nov. 2010, pp. 1-4.

[144] A. Sai, T. Yamaji, and T. Itakura, "A $570 \mathrm{fs}_{r m s}$ integrated-jtter ring-VCO-based 1.21 GHz PLL with hybrid loop," in IEEE Int. Solid-State Circuits Conf. Dig. of Tech. Papers, Feb. 2011, pp. 98-100.

[145] W. Yin, R. Inti, A. Elshazly, B. Young, and P. K. Hanumolu, "A 0.7-to-3.5 GHz 0.6-to-2.8 $\mathrm{mW}$ highly digital phase-locked loop with bandwidth tracking," IEEE. J. Solid-State Circuits, vol. 46, no. 8, pp. 1870-1880, Aug. 2011. 
[146] W. Yin, R. Inti, A. Elshazly, M. Talegaokar, B. Young, and P. K. Hanumolu, "A TDC-less $7 \mathrm{~mW} 2.5$ Gbs digital CDR with linear loop dynamics and offset-free data recovery," IEEE. J. Solid-State Circuits, vol. 46, no. 12, pp. 3163-3173, Dec. 2011.

[147] A. Sai, Y. Kobayashi, S. Saigusa, O. Watanabe, and T. Itakura, "A digitally stabilized type-III PLL using ring VCO with $1.01 \mathrm{ps}_{r m s}$ integrated jitter in $65 \mathrm{~nm}$ CMOS," in IEEE Int. Solid-State Circuits Conf. Dig. of Tech. Papers, Feb 2012, pp. 248-250.

[148] Y. Sun, X. Yu, W. Rhee, S. Ko, W. Choo, B.-H. Park, and Z. Wang, "Low-noise fractional-N PLL design with mixed-mode triple-input LC VCO in 65nm CMOS," in Proc. of the IEEE Radio Freq. Integrated Circuits Symp., May 2010, pp. 61-64.

[149] M. Demirkan, G. Steinbach, K. A. Nishimura, J. P. Keane, and B. E. Wüppermann, "An 8.2 to $20.1 \mathrm{GHz}$ LC PLL with sub-100 fs jitter in $0.13 \mu \mathrm{m}$ SiGe BiCMOS," in Proc. IEEE Compound Semiconductor Integrated Circuit Symp., Oct 2010, pp. 1-4.

[150] M. Wang, B. Zhou, W. Rhee, and Z. Wang, "Continuously auto-tuned and self-ranged dual-path PLL design with hybrid AFC," in Proc. IEEE Conf. on IC Design and Tech., May 2011, pp. 1-4.

[151] M. Ferriss, J.-O. Plouchart, A. Natarajan, A. Rylyakov, B. Parker, J. A. Tierno, A. Babakhani, S. Yaldiz, A. Valdes-Garcia, B. Sadhu, and D. J. Friedman, "An integral path self-calibration scheme for a dual-loop PLL," IEEE. J. Solid-State Circuits, vol. 48, no. 4, pp. 996-1008, Apr. 2013.

[152] B. Sadhu, M. A. Ferriss, A. S. Natarajan, S. Yaldiz, J.-O. Plouchart, A. V. Rylyakov, A. Valdes-Garcia, B. D. Parker, A. Babakhani, S. Reynolds, X. Li, L. Pileggi, R. Harjani, J. A. Tierno, and D. Friedman, "A linearized, low-phase-noise VCO-based 25-GHz PLL with autonomic biasing," IEEE. J. Solid-State Circuits, vol. 48, no. 5, pp. 11381150, Apr. 2013.

[153] W. S. Titus and J. G. Kenney, "A $5.6 \mathrm{GHz}$ to $11.5 \mathrm{GHz}$ DCO for digital dual loop CDRs," IEEE J. of Solid-State Circuits, vol. 47, no. 5, pp. 1123-1130, 2012.

[154] S. P. Bruss and R. R. Spencer, "A 5-GHz CMOS Type-II PLL With Low KVCO and Extended Fine-Tuning Range," IEEE Trans. MTT, vol. 57, no. 8, pp. 1978-1988, Aug 2009.

[155] P.-Y. Wang, J.-H. C. Zhan, H.-H. Chang, and H.-M. S. Chang, "A digital intensive fractional-N PLL and all-digital self-calibration schemes," IEEE. J. Solid-State Circuits, vol. 44, no. 8, pp. 2182-2192, Aug. 2009. 
[156] Y. Sun, J. Qiao, X. Yu, W. Rhee, B.-H. Park, and Z. Wang, "A continuously tunable hybrid LC-VCO PLL with mixed-mode dual-path control and bi-level $\Delta$ - $\Sigma$ Modulated coarse tuning," IEEE Trans. Circuits Syst. I, Reg. Papers, vol. 58, no. 9, pp. 2149-2158, 2011.

[157] Y. Sun, Z. Zhang, N. Xu, M. Wang, W. Rhee, T.-Y. Oh, and Z. Wang, "A 1.75 $\mathrm{mW} 1.1 \mathrm{GHz}$ semi-digital fractional-N PLL with TDC-less hybrid loop control," IEEE Microwave and Wireless Components Letters, vol. 22, no. 12, pp. 654-656, Dec. 2012.

[158] M. Ferriss, B. Sadhu, A. Rylyakov, H. Ainspan, and D. Friedman, "A 12-to-26 GHz fractional-N PLL with dual continuous tuning LC-D/VCOs," in IEEE Int. Solid-State Circuits Conf. Dig. of Tech. Papers, Feb. 2016, pp. 196-198.

[159] X. Zhang, I. Mukhopadhyay, R. Dokania, and A. B. Apsel, "A 46- $\mu$ W self-calibrated gigahertz VCO for low-power radios," IEEE Trans. Circuits and Systems II: Express Briefs, vol. 58, no. 12, pp. 847-851, Dec 2011.

[160] M. H. Perrott, J. C. Salvia, F. S. Lee, A. Partridge, S. Mukherjee, C. Arft, J. Kim, N. Arumugam, P. Gupta, S. Tabatabaei, S. Pamarti, H. Lee, and F. Assaderaghi, "A temperature-to-digital converter for a MEMS-based programmable oscillator with $< \pm 0.5 \%$-ppm frequency stability and < 1-ps integrated jitter," IEEE. J. Solid-State Circuits, vol. 48, no. 1, pp. 276-291, Jan. 2013.

[161] T.-H. Lin and W. J. Kaiser, "A 900-MHz 2.5-mA CMOS frequency synthesizer with an automatic SC tuning loop," IEEE. J. Solid-State Circuits, vol. 36, no. 3, pp. 424-431, Mar. 2001.

[162] A. Atkas and M. Ismail, "CMOS PLL calibration techniques," IEEE Circuits and Devices Magazine, vol. 20, no. 5, pp. 6-11, Sept.-Oct. 2004.

[163] L. Lu, L. Meng, L. Zou, H. Min, and Z. Tang, "A sub- $0.75^{\circ}{ }_{R M S}$-phase-error differentially-tuned fractional-N synthesizer with on-chip LDO regulator and analogenhanced AFC technique," in Proc. IEEE Custom Integrated Circuits Conf., Sept. 2009, pp. 53-56.

[164] B. Zhao, Y. Lian, and H. Yang, "A low-power fast-settling bond-wire frequency synthesizer with fast auto-calibration of loop bandwidth and VCO frequency," IEEE Trans. Circuits Syst. I, Reg. Papers, vol. 60, no. 5, pp. 1188-1199, May 2013. 
[165] W. B. Wilson, U.-K. Moon, K. R. Lakshmikumar, and L. Dai, "A CMOS selfcalibrating frequency synthesizer," IEEE. J. Solid-State Circuits, vol. 35, no. 10, pp. 1437-1444, Oct. 2000.

[166] H. il Lee, J.-K. Cho, K.-S. Lee, I.-C. Hwang, T.-W. Ahn, K.-S. Nah, and B.-H. Park, "A $\Sigma-\Delta$ fractional-N frequency synthesizer using a wide-band integrated VCO and a fast AFC technique for GSM/GPRS/WCDMA applications," IEEE J. of Solid-State Circuits, vol. 39, no. 7, pp. 1164-1169, July 2004.

[167] M. H. Perrott, Y. Huang, R. T. Baird, B. W. Garlepp, D. Pastorello, E. T. King, Q. Yu, D. B. Kasha, P. Steiner, L. Zhang, J. Hein, and B. D. Signore, "A 2.5-Gbs multi-rate 0.25- $\mu \mathrm{m}$ CMOS clock and data recovery cicuit utilizing a hybrid analog/digital loop filter and all-digital referenceless frequency acquisition," IEEE. J. Solid-State Circuits, vol. 41, no. 12, pp. 2930-2944, Dec. 2006.

[168] Y.-C. Yang and S.-S. Lu, "A single-VCO fractional-frequency synthesizer for digital TV tuners," IEEE Trans. Industrial Electronics, vol. 57, no. 9, pp. 3207-3215, Sept. 2010.

[169] J. Shin and H. Shin, "A 1.9-3.8 GHz $\Delta \Sigma$ fractional-N PLL frequency synthesizer with fast auto-calibration of loop bandwidth and VCO frequency," IEEE. J. Solid-State Circuits, vol. 47, no. 3, pp. 665-675, Mar. 2012.

[170] T.-H. Lin and Y.-J. Lai, "An agile VCO frequency calibration technique for a $10-\mathrm{GHz}$ CMOS PLL," IEEE. J. Solid-State Circuits, vol. 42, no. 2, pp. 340-349, Feb. 2007.

[171] J. Lee, K. Kim, J. Lee, T. Jang, and S. Cho, "A 480-MHz to 1-GHz sub-picosecond clock generator with a fast and accurate automatic frequency calibration in $0.13-\mu \mathrm{m}$ CMOS," in Proc. IEEE Asian Solid-State Circuits Conf., Nov. 2007, pp. 67-70.

[172] J. Shin and H. Shin, "A fast and high-precision VCO frequency calibration technique for wideband $\Delta \Sigma$ fractional-N frequency synthesizers," IEEE Trans. Circuits Syst. I, Reg. Papers, vol. 57, no. 7, pp. 1573-1582, Jul. 2010.

[173] A. Kral, F. Behbahani, and A. A. Abidi, "RF-CMOS oscillators with switched tuning," in Proc. IEEE Custom Integrated Circuits Conf., May 1998, pp. 555-559.

[174] J. Cheah, E.-H. Kwek, E. C. Low, C. K. Quek, C. Yong, R. Enright, J. Hirbawi, A. Lee, H. Zie, L. Wei, L. Luong, J. Pan, S.-T. Yang, W. F. A. Lau, and W.-L. Ngai, "Design

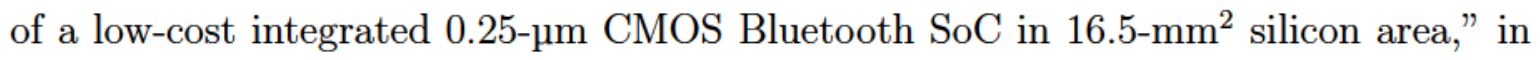
IEEE Int. Solid-State Circuits Conf. Dig. of Tech. Papers, Feb. 2002. 
[175] A. Italia, C. M. Ippolito, and G. Palmisano, "A 1-mW 1.13-1.9 GHz CMOS LC VCO using shunt-connected switched-coupled inductors," IEEE Trans. Circuits and Systems I: Reg. Papers, vol. 59, no. 6, pp. 1145-1155, Jun. 2012.

[176] J.-C. Chien and L.-H. Lu, "Design of a wide-tuning-range millimeter-wave CMOS VCO with a standing-wave architecture," IEEE. J. Solid-State Circuits, vol. 42, no. 9, pp. 1942-1952, Sept. 2007.

[177] A. Bevilacqua, F. P. Pavan, C. Sandner, A. Gerosa, and A. Neviani, "A 3.4-7 GHz transformer-based dual-mode wideband VCO," in Proc. Solid-State circuits Conf., 2006, pp. 440-443.

[178] B. Çatli and M. Hella, "Dual-band ultra-wide tuning range CMOS voltage-controlled oscillator," Elec. Letters, vol. 42, no. 21, pp. 1215-1216, Oct 2006.

[179] G. Cusmai, M. Repossi, G. Albasini, A. Mazzanti, and F. Svelto, "A magnetically tuned quadrature oscillator," IEEE. J. Solid-State Circuits, vol. 42, no. 12, pp. 28702877, Dec. 2007.

[180] Z. Safarian and H. Hashemi, "Wideband multi-mode CMOS VCO design using coupled inductors," IEEE Trans. Circuits and Systems I: Reg. Papers, vol. 56, no. 8, pp. 18301843, Aug. 2009.

[181] P.-L. You and T.-H. Huang, "A switched inductor toplogy using a switchable artificial grounded metal guard ring for wide-FTR MMW VCO applications," IEEE Trans. Electron Devices, vol. 60, no. 2, pp. 759-766, Feb. 2013.

[182] P. Agarwal, P. P. Pande, and D. Heo, "25.3 GHz, $4.1 \mathrm{~mW}$ VCO with $34.8 \%$ tuning range using a switched substrate-shield inductor," in Proc. MTT-S Int. Microwave Symp., 2015.

[183] S. D. Toso, A. Bevilacqua, A. Gerosa, and A. Neviani, "A thorough analysis of the tank quality factor in LC oscillators with switched capacitor banks," in Proc. IEEE Symp. Circuits and Sys., 2010, pp. 1903-1906.

[184] D. Hauspie, E.-C. Park, J. Craninckx, and B. Come, "Wideband VCO with simultaneous switching of frequency band, active core and varactor size," in Proc. European Solid-State Circuits Conf., 2006, pp. 452-455.

[185] E. Vittoz and J. Fellrath, "CMOS analog integrated circuits based on weak inversion operations," Journal of Solid-State Circuits, vol. SC-12, no. 3, pp. 224-231, 1977. 
[186] "Voltage-controlled oscillators," IEEE Radio Frequency Integrated Circuits Virtual J., no. 1, Dec. 2012.

[187] D. Murphy, H. Darabi, and H. Wu, "A VCO with implicit common-mode resonance," in IEEE Int. Solid-State Circuits Conf. Dig. Tech. Papers, Feb. 2015, pp. 442-444.

[188] J. J. Kim, J. Choi, K.-H. Lee, F. Bien, K. Lim, and C.-H. Lee, "Wideband CMOS voltage-controlled oscillator using tunable inductors," Elec. Letters, vol. 46, no. 20, pp. 1391-1393, Sept 2010.

[189] J. W. M. Rogers and C. Plett, Radio Frequency Integrated Circuit Design. Artech House, 2003.

[190] T. O. Dickson, K. H. K. Yau, T. Chalvatzis, A. M. Mangan, E. Laskin, R. Beerkens, P. Westergaard, M. Tazlauanu, M.-T. Yang, and S. P. Voinigescu, "The invariance of characteristic of current densities in nanoscale MOSFETs and its impact on algorithmic design methodologies and design porting of $\mathrm{Si}(\mathrm{Ge})$ (Bi)CMOS high-speed building blocks," IEEE J. Solid State Circuits, vol. 41, no. 8, pp. 1830-1845, Aug. 2006.

[191] E. Hegazi, H. Sj'oland, and A. A. Abidi, "A filtering technique to lower LC oscillator phase noise," IEEE J. of Solid-State Circuits, vol. 36, no. 12, pp. 1921-1930, 2001.

[192] X. Gao, E. A. M. Klumperink, P. F. J. Geraedts, and B. Nauta, "Jitter analysis and a benchmarking figure-of-merit for phase-locked loops," IEEE Trans. Circuits and Systems II: Express Briefs, vol. 56, no. 2, pp. 117-121, Feb. 2009.

[193] R. T. Stadius, L. Xu, S. Lindfors, R. Kaunisto, A. Parssinen, and J. Ryynanen, "A digital frequency synthesizer for cognitive radio spectrum sensing applications," Transactions on Microwave Theory and Techniques, vol. 58, no. 5, pp. 1339-1348, 2010.

[194] J. Kim, S. J. Lee, S. Kim, J. O. Ha, Y. S. Eo, and H. Shin, "A 54-862-MHz CMOS transceiver for TV-band white-space device applications," Transaction on Microwave Theory and Techniques, vol. 59, no. 4, pp. 966-977, 2011.

[195] P. Nuzzo, K. Vengattaramane, M. Ingels, V. Giannini, M. Steyaert, and J. Craninkx, "A 0.1-5 GHz dual-VCO software-defined sigma-delta frequency synthesizer in $45 \mathrm{~nm}$ digital CMOS," in RFIC Symp Proc, June 2009, pp. 321-324.

[196] C.-Y. Yang, H.-M. Wu, J.-H. Weng, and P.-H. Wu, "A wide-range frequency synthesizer using a compensated phase-rotating technique for digital TV tuners," in Int. Symp. ISPACS Proc., Dec 2009, pp. 397-400. 
[197] K.-C. Peng, C.-H. Lee, C.-H. Chen, and T.-S. Horng, "Enhancement of frequency synthesizer operating range using a novel frequency-offset technique for LTE-A and CR applications," Transactions on Microwave Theory and Techniques, vol. 61, no. 3, pp. 1215-1223, 2013.

[198] J. G. Maneatis, J. Kim, I. McClatchie, J. Maxey, and M. Shankaradas, "Self-biased high-bandwidth low-jitter 1-to-4096 multiplier clock generator PLL," IEEE. J. SolidState Circuits, vol. 38, no. 11, pp. 1795-1803, Nov. 2003.

[199] Y.-C. Yang and S.-S. Lu, "A single-VCO fractional-N frequency synthesizer for digital TV tuners," IEEE Trans. Industrial Electronics, vol. 59, no. 9, pp. 3207-3215, Sept. 2010.

[200] D. Baek, T. Song, E. Yoon, and S. Hong, "8-GHz CMOS quadrature VCO using transformer-based LC tank," IEEE Microwave and Wireless Components Letters, vol. 13 , no. 10 , pp. 446-448, Oct. 2003.

[201] M.-T. Hsu and Y.-H. Huang, "A low phase noise and low power CMOS VCO with transformer feedback," in Proc. IEEE Asia Pacific Microwave Conf., Dec. 2009.

[202] C.-C. Li, T.-P. Wang, C.-C. Kuo, M.-C. Chuang, and H. Wang, "A 21 GHz complementary transformer coupled CMOS VCO," IEEE Microwave and Wireless Components Letters, vol. 18, no. 4, pp. 278-280, Apr. 2008.

[203] K. Kwok and H. C. Luong, "Ultra-low-voltage high performance CMOS VCOs using transformer feedback," IEEE J. Solid State Circuits, vol. 40, no. 3, pp. 652-660, Mar. 2005.

[204] N.-J. Oh and S.-G. Lee, "11-GHz CMOS differential VCO with back-gate transformer feedback," IEEE Microwave and Wireless Components Letters, vol. 15, no. 11, pp. 733-735, Nov. 2005.

[205] A. Ravi, K. Soumyanath, R. E. Bishop, B. A. Bloechel, and L. R. Carley, "An optimally transformer coupled, $5 \mathrm{GHz}$ quadrature VCO in a 0.18 - $\mu \mathrm{m}$ digital CMOS process," in Symp. VCLI Circuits Dig. Tech Papers, Jun. 2003, pp. 141-144.

[206] C. C. Meng, Y. W. Chang, and S. C. Tseng, "4.9-GHz low-phase-noise transformerbased superharmonic-coupled GaInP/GaAs HBT QVCO," IEEE Microwave and Wireless Components Letters, vol. 16, no. 6, pp. 339-341, Jun. 2006. 
[207] S. Ko, J.-G. Kim, T. Song, E. Yoon, and S. Hong, "20 GHz integrated CMOS frequency sources with a quadrature VCO using transformers," in IEEE RFIC Symp Dig., June 2004.

[208] A. Bevilacqua, F. P. Pavan, C. Sandner, A. Gerosa, and A. Neviani, "Transformerbased dual-mode voltage-controlled oscillators," IEEE Trans. Circuits and Sys. II: Express Briefs, vol. 54, no. 4, pp. 293-297, Apr. 2007.

[209] M. Straayer, J. Cabanillas, and G. M. Rebeiz, "A low-noise transformer-based $1.7 \mathrm{GHz}$ CMOS VCO," in IEEE Int. Solid-State Circuits Conf. Dig. of Tech. Papers, Feb. 2002.

[210] S.-J. Yun, N. D. B. Yen, I. Lee, J.-T. Lee, and S.-G. Lee, "A differentially-tuned voltage controlled oscillator using symmetric transformer," IEEE Microwave and Wireless Components Letters, vol. 18, no. 7, pp. 464-466, Jul. 2008.

[211] M. Babaie and R. B. Staszewski, "A class-F CMOS oscillator," IEEE J. Solid State Circuits, vol. 48, no. 12, pp. 3120-3133, Dec. 2013.

[212] A. Goel and H. Hashemi, "Frequency switching in dual-resonance oscillators," IEEE J. Solid State Circuits, vol. 42, no. 3, pp. 571-582, Mar. 2007. 